\title{
WestVirginiaUniversity
}

THE RESEARCH REPOSITORY @ WVU

Graduate Theses, Dissertations, and Problem Reports

2013

\section{Abelson Kinase Based Regulation of Tumor Cell Invasion in HNSCC}

Karen E. Hayes

West Virginia University

Follow this and additional works at: https://researchrepository.wvu.edu/etd

\section{Recommended Citation}

Hayes, Karen E., "Abelson Kinase Based Regulation of Tumor Cell Invasion in HNSCC" (2013). Graduate Theses, Dissertations, and Problem Reports. 472.

https://researchrepository.wvu.edu/etd/472

This Dissertation is protected by copyright and/or related rights. It has been brought to you by the The Research Repository @ WVU with permission from the rights-holder(s). You are free to use this Dissertation in any way that is permitted by the copyright and related rights legislation that applies to your use. For other uses you must obtain permission from the rights-holder(s) directly, unless additional rights are indicated by a Creative Commons license in the record and/ or on the work itself. This Dissertation has been accepted for inclusion in WVU Graduate Theses, Dissertations, and Problem Reports collection by an authorized administrator of The Research Repository @ WVU.

For more information, please contact researchrepository@mail.wvu.edu. 


\title{
Abelson Kinase Based Regulation of Tumor Cell Invasion in HNSCC
}

\section{Karen E. Hayes}

Dissertation Submitted to the School of Medicine at West Virginia University In Partial Fulfillment of the Requirements for the Degree of

Doctor of Philosophy in Cancer Cell Biology

\author{
Steven Frisch, Ph.D., Chair \\ Laura Gibson, Ph.D. \\ Jun Liu, Ph.D. \\ Linda Vona-Davis, Ph.D. \\ Robert Wysolmerski, Ph.D. \\ Scott Weed, Ph.D., Mentor \\ Cancer Cell Biology Program \\ Morgantown, West Virginia \\ 2012
}

Keywords: Src, Abl, Erk, invasion, invadopodia, cortactin, HNSCC Copyright 2012 Karen E. Hayes 


\begin{abstract}
Abelson Kinase Based Regulation of HNSCC Tumor Cell Invasion
\end{abstract}

Karen E. Hayes

Nearly 40,000 new cases of head and neck squamous cell carcinoma (HNSCC) are annually diagnosed in the United States. The current standard of care for HNSCC patients consists of chemoradiation often combined with targeted therapeutic agents. However, the five-year survival rate for patients that relapse is $<50 \%$. Patients with recurrent or metastatic disease have a very poor prognosis and typical survive $<12$ months due to therapeutic resistance and loco-regional invasive spread. HNSCC invasion is mediated in part by lamellipodia and invadopodia, two actin-based structures responsible for facilitating invasive movement. Key signaling pathways that govern lamellipodia and invadopodia production are hyperactivated or overexpressed in HNSCC, including EGFR, Src, Erk 1/2, and cortactin. The overall goal of this dissertation is to determine how these signaling components regulate lamellipodia and invadopodia production and function in HNSCC. Three studies were completed that address these issues. Study One reveals an anti-invasive function for Abl in invadopodia regulation in HNSCC, results that are contrary to the pro-invasive influence $\mathrm{Abl}$ has in breast and other cancer types. Study Two establishes a fundamental role for Src has in HNSCC invasion and metastasis through the use of the dual Src/Abl inhibitor saracatinib. In Study Three we determine the expression and activation levels of Erk 1/2 in HNSCC patient samples and the role of Erk 1/2 cortactin phosphorylation in HNSCC adhesion, migration and lamellipodia persistence. Collectively these studies shed new light into the fundamental mechanisms utilized during various steps in HNSCC invasion, providing the potential for refinement and development of new avenues for therapeutic intervention. 


\section{Acknowledgements}

Well I finally have reached the end of my journey; it has been a long and bumpy road. This is by no means a road that I have travel on my own, throughout my time at West Virginia University I have meet many kind people who have helped me along my way. I would like to take this time to express my gratitude to the people who have enriched my life here at WVU. First, I would like say that I am sincerely gratefully to the graduate school at WVU for giving me this opportunity, I would never been able to obtain a PhD without the financial assistance this program has provided. I would also like to thank my committee members; you have pushed me to become a better research scientist, thank you for your insights and recommendations throughout my graduate career. Next, I wish to express my sincerest gratitude to my mentor, Dr. Scott Weed, his guidance, instruction and patience have allowed me to develop as a scientist. It may have been a struggle but I think that the training wheels can now come off now.

This brings me to all the members of the Weed laboratory past and present, thank you for your assistance great or small I will remember you always. I would like to mention a few members personally, Jason Evans where would I be without you, probably lost in the labyrinth of halls at WVU, it has be a long haul but we made it. I promise not to take your scissors ever again. Dr. Amanda Ammar (aka Arnold Hammer), you have been such a good friend. Thank you for caring enough to make sure I wasn't being eaten by my cats. Lesly Anne Lopez-Skinner, you may have moved many miles away to Texas many years ago but you are still one of my closest friends. I hope our career paths will cross again because you are one of the few people I know that is just pure goodness. Elyse Walk, my IT guru, what will I do when you are not there to help me with my computer problems. Steve Markwell, no one has ever made me laugh like you, just try not to burn down the laboratory after I'm gone. Finally to our honorary Weed lab member, Tammy Whitacre, you give so much of yourself to others; I can only hope to be as giving. Also, you need to remember to take some time for yourself, take that vacation I'm always telling you to take.

I would also like to thank the members of my family for their support. To my mother, who has always been there to listen to my ranting and raving, nodding and agreeing with me even though I'm completely in the wrong. I know I have you to thank for my stubbornness that has kept me going even through the worst of the times. To my father; who is no longer on this mortal plain, you were always there for me even when you didn't agree with what I was doing. Thank you both for being my safety net. To my sister, we have traveled this bumpy collegiate road often together. You have been there to lend a helping hand without question; I think the time we had the tub stuck on the stairs will be my most memorable moment. Thank you so much, you will never know how truly grateful I am. To my brother, yes I have finally made it; you can stop asking me "When are you going to graduate?". 


\section{$\underline{\text { Table of Contents }}$}

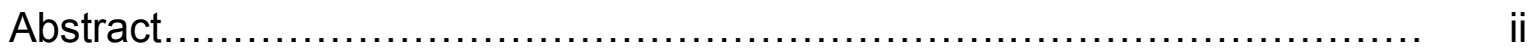

Acknowledgements.........................................................

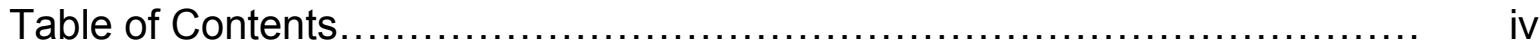

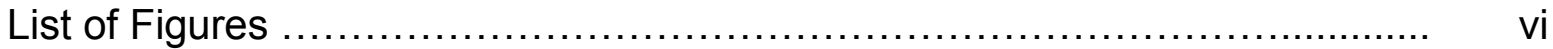

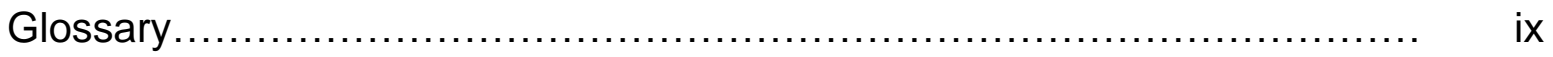

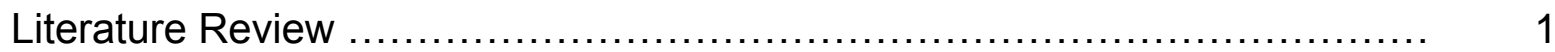

Head and neck squamous cell carcinoma Methods of cancer cell motility and invasion Cortactin: A node of actin network regulation EGFR: Signaling and significance in HNSCC

Erk1/2: Effector proteins for EGFR signaling

Src: The first oncogene

Abl: A kinase with dichotomous roles

Study 1: Ableson Kinases Negatively Regulate Invadopodia Function and Invasion in Head and Neck Squamous Cell Carcinoma by Inhibiting an HB-EGF Autocrine Loop

Study 2: Saracatinib Impairs Head and Neck Squamous Cell Carcinoma Invasion by Disrupting Invadopodia Function 109

Study 3: Cortactin Phosphorylated by ERK1/2 Localizes to Sites of Dynamic Actin Regulation and Is Required for Carcinoma Lamellipodia Persistence.

General Discussion 192 Appendix

Quantitative Measurement of Invadopodia-mediated Extracellular Matrix Proteolysis in Single and Multicellular Contexts. 
Multi-photon Imaging of Tumor Cell Invasion in an Orthotopic Mouse Model of Oral Squamous Cell Carcinoma ............................. 215

Revisiting the ERK/Src Cortactin Switch ............................ 223

Further Insights into Cortactin Conformational Regulation ............. 226

Oncogenic Src Requires a Wild-type Counterpart to Regulated

Invadopodia Maturation …....................................... 229

Curriculum Vitae..................................................... 245 


\section{List of Figures and Tables}

\section{Literature Review}

Figures

1. Plasticity of tumor cell migration.

2. Schematic diagram of cortactin structure with adapter and signaling partners.

3. Schematic diagram of EGFR and conical MAPK signaling pathways.

4. Erk1/2 signaling cascade and regulation of cellular processes.

5. Schematic diagram of Src, activation and downstream signaling pathways.

6. Schematic diagram of Abl structure, activation and downstream signaling pathways.

Table

1. Table of cortactin $\mathrm{SH} 3$ binding proteins

\section{Study 1}

1 Abl expression inhibits invadopodia activity in HNSCC cells.

2 Targeted inhibition of Abl family kinases with imatinib has divergent effects on invadopodia activity in HNSCC and breast cancer cells.

3 Imatinib enhances HNSCC invasion through 3D collagen matrices.

4 Elevated Src activity bypasses Abl family kinase regulation of invadopodia function.

5 Imatinib activates the invadopodia kinase pathway in HNSCC cells.

6 Imatinib induced HB-EGF stimulates HNSCC invadopodia ECM degradation.

7 Targeted inhibition of the EGFR-Src pathway impairs HNSCC invadopodia activity.

8 Schematic diagram of EGFR-invadopodia signaling pathways altered by Abl 
inhibition in HNSCC.

Supplemental Figures:

1 Abl knockdown does not impact the number of HNSCC invadopodia.

2 Abl knockdown does not impact matrix degradation in MDA-MB-231 cells.

3 Determination of PDGFRa expression levels in OSC19, UMSCC1 and MDAMB-231 cells.

$4 \mathrm{Abl}$ and Arg expression levels in wild-type (WT) and Abl-//Arg-/- (DKO) mouse embryo fibroblasts (MEFs).

5 Evaluation of imatinib on EGFR and Crk signaling.

6 Targeted inhibition of the EGFR-Src pathway.

7 Saracatinib impairs invadopodia activity in HNSCC and MDA-MB-231 cells.

\section{Study 2}

1. Effects of saracatinib on HNSCC proliferation, cell cycle progression, Erk1/2 activation and in vitro invasion.

2. Saracatinib inhibits Src activity and downstream Src substrate phosphorylation in HNSCC cell lines.

3. Saracatinib inhibits Src activity, perineural invasion and cervical lymph node metastasis in orthotopic UMSCC1 tongue tumors.

4. Saracatinib inhibits invadopodia formation and ECM degradation.

5. MMP9 secretion and ECM degradation activity in HNSCC cells is blocked by saracatinib.

Supplemental Figures:

6. Specificity of human anti-pY421 cortactin antibody. 


\section{Study 3}

1. Specificity and validation of pS405 and pS418 phospho-specific cortactin antibodies.

2. Growth factor-stimulated Erk 1/2 activation mediates phosphorylation of cortactin at serine 405 and 418.

3. EGF-induced conversion of cortactin from $80 \mathrm{kDa}$ to $85 \mathrm{kDa}$ is impaired by Src and MEK1/2 inhibition.

4. Cortactin tyrosine and serine phosphorylation resultant of v-Src activation are not interdependent.

5. Targeted inhibition of MEK1/2 inhibits HNSCC cell motility.

6. Cortactin phosphorylation at serine 405 and 418 regulates carcinoma cell migration and adhesion.

7. Cortactin phosphorylation at serine 405 and 418 is required for lamellipodia persistence. 


\section{Glossary}

\begin{tabular}{|c|c|}
\hline A & alanine \\
\hline$\AA$ & angstrom \\
\hline Abl (Abl1, c-Abl) & abelson tyrosine kinase \\
\hline $\mathrm{ABP}$ & actin binding protein \\
\hline ACK1 & activated Cdc42-associated kinase \\
\hline ADAM & a disintegrin and metalloproteinase \\
\hline ADP & adenosine diphosphate \\
\hline AFAP110 & actin filament-associated protein of $110 \mathrm{kDa}$ \\
\hline Akt (PKB) & protein kinase B \\
\hline AMAP1 (ASAP1) & AMY-1-binding protein 1 \\
\hline ANOVA & analysis of variance \\
\hline Arf6 & ADP-ribosylation factor 6 \\
\hline $\operatorname{Arg}(\mathrm{Abl2})$ & Abelson-related gene \\
\hline Arp2/3 & actin related protein $2 / 3$ \\
\hline AREG & amphiregullin \\
\hline ATM & ataxia telangiectasia mutated protein \\
\hline ATP & adenosine triphosphate \\
\hline BAX & Bcl-2-associated $\mathrm{X}$ protein \\
\hline $\mathrm{Bcl}-2$ & B-cell lymphoma 2 \\
\hline BCR & breakpoint cluster region \\
\hline BCR-Abl & breakpoint cluster region- ableson \\
\hline bFGF & basic fibroblast growth factor \\
\hline BPGAP1 & $\mathrm{BCH}$ domain-containing Cdc42GAP-like protein \\
\hline BSA & bovine serum albumin \\
\hline C & Celsius \\
\hline CAFs & cancer associated fibroblasts \\
\hline CAS & Crk-associated substrate \\
\hline Cbl & Casitas B-lineage lymphoma gene \\
\hline CBP90 & cortactin-binding protein 90 \\
\hline CD2AP & CD2- associated protein \\
\hline Cdc42 & cell division cycle 42 , GTP binding protein \\
\hline CDK2 & cyclin-dependent kinase 2 \\
\hline CEF & chicken embryo fibroblast \\
\hline Cer & cerulean fluorescent protein \\
\hline CFP & cyan fluorescent protein \\
\hline CIN85 & Cbl-interacting $85-k D a$ protein \\
\hline CIP4 & Cdc42-interacting protein \\
\hline c-kit & cellular homologue of the feline sarcoma viral oncogene v-kit \\
\hline c-Met & MNNG HOS transforming gene ; hepatocyte growth factor receptor \\
\hline CML & chronic mylogenous leukemia \\
\hline CMV & cytomegalovirus promoter \\
\hline CortBP1 & cortactin binding protein 1 \\
\hline $\mathrm{CP}$ & capping protein \\
\hline Crk I/II & Cdc2 related kinase $1 / 2$ \\
\hline
\end{tabular}




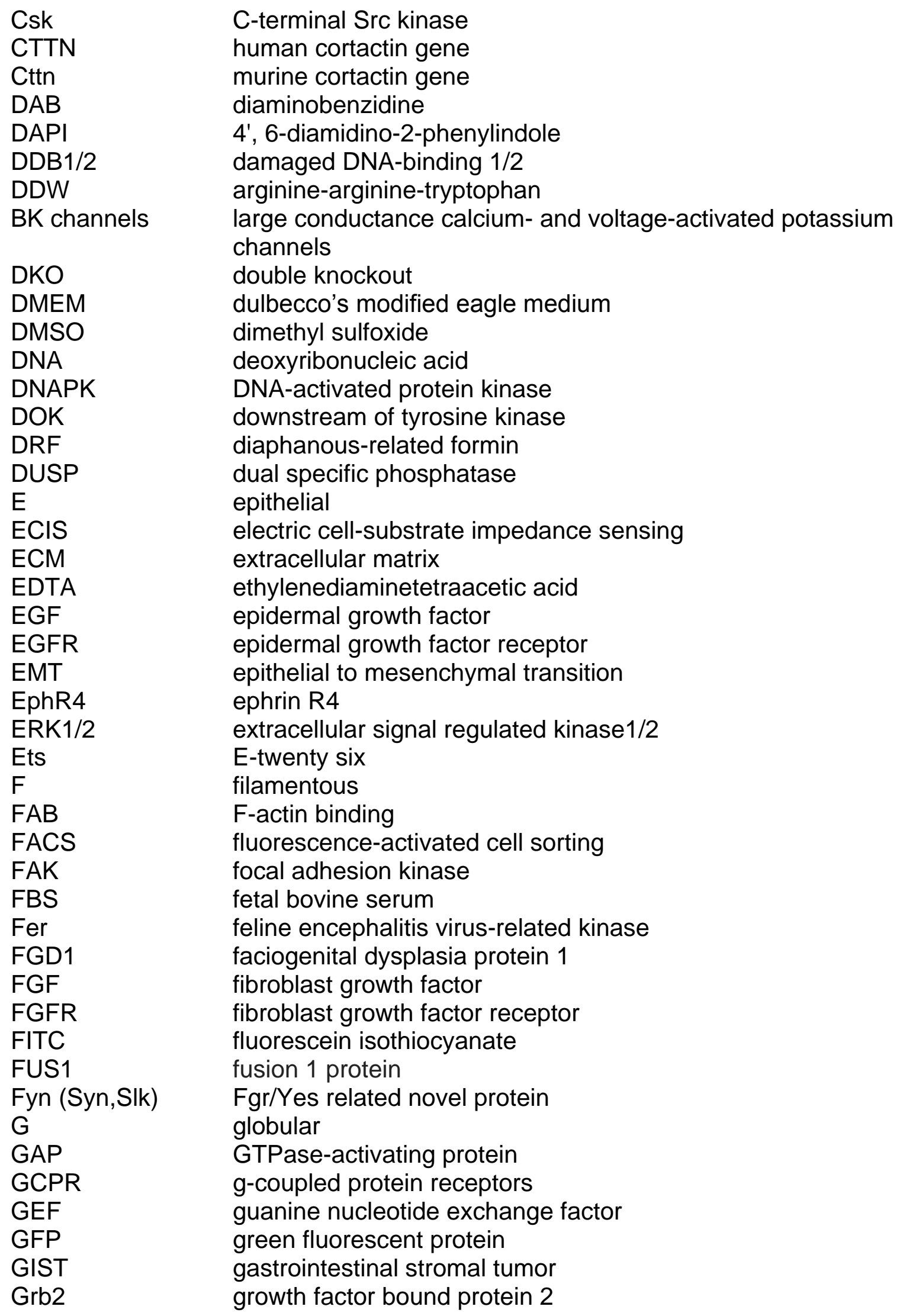




$\begin{array}{ll}\text { GTP } & \text { guanosine-5'- triphosphate } \\ \text { HA-tagged } & \text { hemaglutinin tagged } \\ \text { HDAC1 } & \text { histone deacetylases 1 } \\ \text { H \& E } & \text { hematoxylin and eosin } \\ \text { HER } & \text { Human Epidermal Growth Factor Receptor } \\ \text { HGF } & \text { hepatocyte growth factor } \\ \text { HIP1R } & \text { Huntingtin-interacting protein1 related } \\ \text { HP } & \text { helical proline } \\ \text { HNSCC } & \text { head and neck squamous cell carcinoma } \\ \text { IGFR } & \text { insulin-like growth factor 1 receptor } \\ \text { IQGAP } & \text { IQ motif containing GTPase-activating protein } \\ \text { IHC } & \text { immunohistochemistry } \\ \text { JAK } & \text { Janus kinase } \\ \text { JNK } & \text { C-Jun N-terminal kinase } \\ \text { kDa } & \text { kiloDalton } \\ \text { K-RAS } & \text { Kirsten rat sarcoma viral oncogene homolog } \\ \text { KSR1 } & \text { kinase suppressor of Ras1 } \\ \text { LSP1 } & \text { lymphocyte-specific protein 1 } \\ \text { MAPK } & \text { mitogen-activated protein kinase } \\ \text { mCh } & \text { mCherry fluorescent protein } \\ \text { MDM2 } & \text { murine double minute } 2 \\ \text { MEF } & \text { mouse embryonic fibroblast } \\ \text { MEK1/2 } & \text { MAP kinase kinase 1/2 } \\ \text { MIM } & \text { missing in metastatis } \\ \text { MIBs } & \text { multiplexed inhibitor beads } \\ \text { MLCII } & \text { myosin light chain II } \\ \text { MLCK } & \text { myosin light chain kinase } \\ \text { MMP } & \text { matrix metalloprotease } \\ \text { Mr } & \text { mass relative } \\ \text { MT-MMP } & \text { matrix bound matrix metalloprotease } \\ \text { MT1-MMP } & \text { matrix bound matrix metalloprotease 1 } \\ \text { mTor } & \text { mammalian target of rapamycin } \\ \text { MTT } & \text { 3-(4,5-dimethylthiazol-2-yl)-2,5-diphenyltetrazolium bromide } \\ \text { NADPH } & \text { nicotinamide adenine dinucleotide phosphatase } \\ \text { NCK } & \text { non-catalytic region of tyrosine kinase adaptor protein } \\ \text { NF-KB } & \text { nuclear factor-kB } \\ \text { NI } & \text { not infected } \\ \text { NOXA } & \text { NADPH oxidase activator } \\ \text { NPF } & \text { nucleation-promoting factor } \\ \text { NSCLC } & \text { non-small cell lung carcinoma } \\ \text { NTA } & \text { N-terminal acidic domain } \\ \text { N-WASp } & \text { Neuronal Wiskott-Aldrich Syndrome Protein } \\ \text { p120RASGAP } & \text { Ras GTPase activating protein, 120 kDa } \\ \text { p190RhoGAP } & \text { Rho GTPase activating protein, 190 kDa } \\ \text { Pak1 } & \\ \text { PAK3 } & \text { p21-activated protein kinase } \\ & \end{array}$




$\begin{array}{ll}\text { PBS } & \text { phosphate buffered saline } \\ \text { PCAF/p300 } & \text { P300/ CREB-binding protein-associated factor } \\ \text { PDGF } & \text { platelet-derived growth factor } \\ \text { PDGFR } & \text { platelet-derived growth factor receptor } \\ \text { PI3K } & \text { phosphoinositide-3 kinase } \\ \text { PIP2 } & \text { phosphatidylinositol-4,5-bisphosphate } \\ \text { PIP3 } & \text { phosphatidylinositol 3, 4, 5-triphosphate } \\ \text { PKC } & \text { protein kinase C } \\ \text { PKD } & \text { protein kinase D } \\ \text { PLC } & \text { phospholipase C } \\ \text { PLCY } & \text { phospholipase-C gamma-1 } \\ \text { PLL } & \text { poly-L-lysine } \\ \text { PP2A } & \text { protein phosphatase 2A } \\ \text { PP2C } & \text { protein phosphatase 2C } \\ \text { PRR } & \text { proline-rich region } \\ \text { PSS } & \text { phosphate saline solution } \\ \text { PTB } & \text { phosphotyrosine binding domain } \\ \text { PTPa } & \text { protein tyrosine phosphatase a } \\ \text { PTP1B } & \text { protein tyrosine phosphatase 1B } \\ \text { PTPPEST } & \text { protein tyrosine phosphatase-PEST } \\ \text { PTPN1 } & \text { protein tyrosine phosphatase, nonreceptor-type 1 } \\ \text { PUMA } & \text { p53 upregulated modulator of apoptosis } \\ \text { Pyk2 } & \text { proline-rich tyrosine kinase 2 } \\ \text { R } & \text { arginine } \\ \text { Rab7/8 } & \text { Ras-associated binding 7/8 } \\ \text { Rac } & \text { Ras-related C3 botulinum toxin substrate 1 } \\ \text { RAF } & \text { Rapidly Accelerated Fibrosarcoma oncogene } \\ \text { Rb } & \text { retinoblastoma protein } \\ \text { RhoA } & \text { Ras homolog family member A } \\ \text { RhoGAP } & \text { Rho family GTPase-activating protein } \\ \text { RIPA } & \text { radioimmunoprecipitation assay } \\ \text { RNAi } & \text { RNA interference } \\ \text { ROCK } & \text { Rho-associated serine/threonine kinase } \\ \text { ROls } & \text { reactive oxygen intermediates } \\ \text { ROS } & \text { reactive oxygen species } \\ \text { RSV } & \text { Rous sarcoma virus } \\ \text { RTK } & \text { receptor tyrosine kinase } \\ \text { S } & \text { serine } \\ \text { SDS-PAGE } & \text { sodium dodecyl sulfate polyacrylamide gel electrophoresis } \\ \text { SEF } & \text { similar expression to fgf genes } \\ \text { SFK } & \text { Src family kinases } \\ \text { SH1 } & \text { tyrosine kinase } \\ \text { SH2 } & \text { Src homology 2 } \\ \text { SH3 } & \text { Src homology 3 } \\ \text { SH4 } & \text { SHc homology 4 } \\ \text { SHANK } & \end{array}$




$\begin{array}{ll}\text { Shc } & \text { Src homology 2 containing } \\ \text { SHP-2 } & \text { Src homology 2-containing tyrosine phosphatase } \\ \text { SiRNA } & \text { small interfering ribonucleic acid } \\ \text { SIRT1 } & \text { silent mating type information regulation } 2 \text { homolog 1 (sirtuin) } \\ \text { SNARE } & \text { soluble NSF attachment protein receptors } \\ \text { SOS } & \text { son of sevenless } \\ \text { Src } & \text { sarcoma kinase } \\ \text { STAT } & \text { signal transducer and activator of transcription } \\ \text { SYF } & \text { Src-/-Yes-/-Fyn-/- } \\ \text { SYF+/+ } & \text { Src+/+ Yes-/-Fyn-/- } \\ \text { Syk } & \text { spleen tyrosine kinase } \\ \text { Tg } & \text { transgenic } \\ \text { TGFa } & \text { transforming growth factor a } \\ \text { TGF } \beta & \text { transforming growth factor } \beta \\ \text { Tks5 } & \text { five SH3 domain (fish)/SH3 and PX domains protein 2A } \\ \text { TPM } & \text { triple point mutant } \\ \text { TRITC } & \text { tetramethyl rhodamine iso-thiocyanate } \\ \text { Trp53 } & \text { transformation related protein 53 gene } \\ \text { tSLa29 } & \text { temperature-sensitive viral Src clone 29 } \\ \text { TYM } & \text { triple tyrosine mutant } \\ \text { UPAR } & \text { urokinase type plasminogen activator } \\ \text { VAbl } & \text { viral Ableson } \\ \text { VASP } & \text { vasodilator-stimulated phosphoprotein } \\ \text { VDAC } & \text { voltage-dependent anion channel } \\ \text { VEGF } & \text { vascular endothelial growth factor } \\ \text { V-Src } & \text { viral sarcoma gene } \\ \text { W } & \text { tryptophan } \\ \text { WASp } & \text { Wiscott Aldrich Syndrome protein } \\ \text { WAVE } & \text { WASp family verprolin-homologous protein } \\ \text { WH2 } & \text { WASp homology domain } \\ \text { WIP } & \text { WASp interacting protein } \\ \text { WT } & \text { wild-type } \\ \text { Y } & \text { tyrosine } \\ \text { ZO-1 } & \text { Zonula occludens protein 1 } \\ & \\ & \end{array}$




\section{Literature Review}

\section{Head and neck squamous cell carcinoma.}

Head and neck squamous cell carcinoma (HNSCC) arises in the mucosal epithelial linings of the oral cavity, oropharynx, nasopharynx, larynx and hypopharynx $(1,2)$. In the United States approximately 40,000 patients are diagnosed with HNSCC each year, comprising $3 \%$ to $5 \%$ of all cancer patients (3). HNSCC can be classified as human papilloma virus (HPV)-positive or HPV-negative. The major risk factors of HPVnegative HNSCC are sustained tobacco and alcohol use, with $90 \%$ of HNSCC cases having a positive correlation. Recently tobacco-associated HNSCC diagnoses have declined, likely due to the decrease in tobacco consumption in the United States over the last eleven years. Conversely, the number of HPV-positive HNSCC patients continues to increase. (3-5).

Presently, HPV-positive (predominantly HPV type-16) HNSCC patients comprise approximately $20 \%$ of all HNSCC cases, of which $60 \%$ to $80 \%$ are oropharyngeal-based cancers (5). HPV is a small circular double stranded DNA virus encoding sequences for early proteins E1, E2 and E4-E7 and late proteins L1 and L2. HPV transforms epithelial cells via E6- and E7-mediated regulation of tumor suppressors, p53 and Rb and other key components while E5 modulates epidermal growth factor receptor (EGFR) and platelet-derived growth factor receptor (PDGFR) internalization $(5,6)$. In general, HPVpositive HNSCC primary tumors are smaller, poorly differentiated and have a 5-year survival rate between $70-80 \%$. This survival rate is significantly better than the 5 -year rate for HPV-negative HNSCC, which is less than $50 \%$. Both HNSCC sub-types receive the same standard treatment consisting of tumor resection, post-operative irradiation therapy and/or platinum-based chemotherapy $(3,4,7)$. Regardless of HPV designation, recurrent and/or metastatic HNSCC patients have an abysmally poor prognosis with a median survival rate of less than one year despite the addition of concomitant treatment with the EGFR-targeted pharmacological agent cetuximab $(4,8)$.

In general HNSCC presents as a locoregional disease involving metastasis to cervical lymph nodes, but distant metastases arise occasionally in lungs, bone, or liver of 
HNSCC patients (9). Metastasis is a multi-faceted processes, involving invasion through the basement membrane and extracellular matrix (ECM), intravasation and extravasion through vascular endothelial layers, and colonization at distant sites $(9,10)$. The heterogeneity of tumors and the complexity of the microenvironment perpetuate a multitude of proposed modes of cell invasion, from collective to single cell amoeboid/mesenchymal phenotypes (Figure 1) (11).

\section{Methods of cancer cell motility and invasion.}

Amoeboid invasion (blebbing or pseudopodal) is generated though physical force that permits rounded, poorly attached individual tumor cells to push through gaps in loose connective tissue and induce non-proteolytic deformation of the extracellular matrix. Amoeboid invasion utilizes bleb-like membrane protrusions, pseudopodia (Rac GTPase induced cylindrical actin-network based membrane protrusions), or filopodia (Cdc42 GTPase mediated slender membrane protrusions containing cross-linked parallel filamentous ( $F$ )-actin bundles at the leading edge of polarized motile cells. These structures assist in mediating the speed and trajectory of the cell during amoeboid

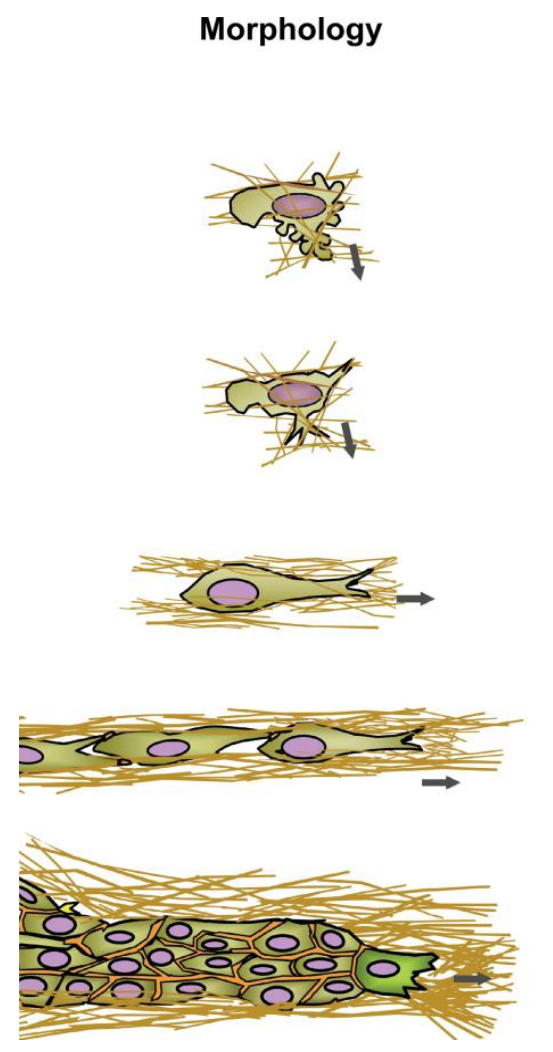

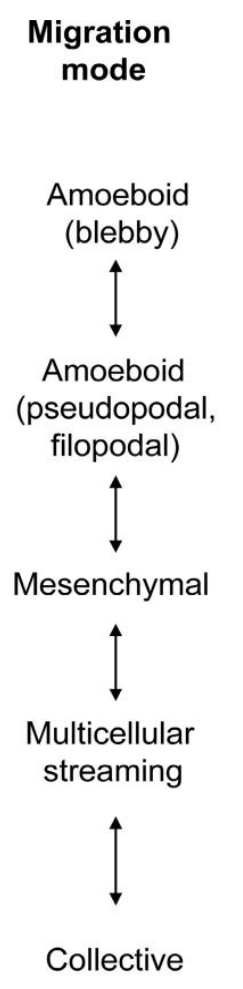

Figure 1 Plasticity of tumor cell migration. Modes of tumor cell migration are classified by cellular morphology and proteolytic activity. Individual cell movements: amoeboid mediated by protease independent and contractile forces pushing through loose connective tissue, or mesenchymal mediated by protease-dependent degradation of extracellular matrix. Collective cell movements: multicellular streaming mediated by chemotactic signaling and pathways generated by matrix remodeling, or collective movement reliant focalized ECM degradation and cell-to-cell contacts to generate directionality.

Friedl, P. and Wolf, K. (2010) J. Cell. Biol., 188(1), 11-19 (modified). 
movement (11-13). The myosin II influenced contractile forces utilized to push the cell forward are generated via Rho-mediated activation of the Rho-associated serine/threonine kinase (ROCK), resulting in simultaneous inactivating phosphorylation of myosin phosphatase and subsequent persistent phosphorylation of myosin light chain on threonine 18 and serine 19 to collectively drive cell contraction $(14,15)$.

Mesenchymal motility is a multi-step process dependent on proteolytic activity to remodel connective tissue. Spindle shaped cells in response to spatial and temporal signaling extend actin-based protrusion classified as invadopodia. Invadopodia are proteolytic actin-rich structures that degrade the ECM or basement membrane. Invadopodia-generated motility occurs via actomysin-mediated contraction that propels the cell forward along with retraction of cell posterior $(16,17)$. The plasticity of tumor cells allows transition from amoeboid and mesenchymal invasion (and vice versa) based on microenvironment conditions as well as intra- and extracellular signaling cues $(11,18)$.

Collective cell movement encompasses invading cellular streams and cellular sheets that maintain cell-cell contact with each other and possess focalized degradation of ECM at the invasive front. Cellular stream invasion utilizes cancer associated fibroblasts (CAF) or mesenchymal tumor cells to generate microtracks in connective tissue, permitting chain-like migration (19). The intercellular signaling generated from the cell-cell contact directs cellular collective movement (20). Collective invasion is the primary invasion mode utilized by HNSCC (18).

This dissertation focuses on two actin-generated organelles, lamellipodia and invadopodia, that are necessary for these modes of invasion. Lamellipodial-based 2D invasion occurs at the leading edge of motile cells and possess little to no proteolytic activity. Lamellipodia are dynamic structures that last only minutes in vitro; they are responsible for facilitating rapid directional movement. Conversely, invadopodial-based $2 \mathrm{D}$ invasion is mediated by proteolytic degradation of ECM on the ventral surface of the cell. Invasion occurring via invadopodia is generally slow, with invadopodia structures lasting up to several hours (21). Lamellipodia (or pseudopodia; the 3D equivalent) are thin sheet-like membrane protrusions induced by extrinsic chemokines or adhesion 
signaling that function by attaching to the ECM and pulling the cell forward. The prototypical Rho-family GTPases Rac1, RhoA and Cdc42, as well as receptor tyrosine kinases and phospholipids propagate cytoplasmic signaling responsible for stimulating branched actin-based cytoskeletal networks generating the force required to extend the membrane (22,23). Actin nucleating promoting factors (NPFs) are responsible for lamellipodia extension, where the actin-related protein 2/3 (Arp 2/3) complex forms branched actin networks, while NPFs of the formin family (e.g.; mDia1), and Spire (Cordon Blue) facilitate straight actin filament formation that serves as the basis for subsequent branched Arp2/3-produced networks $(24,25)$.

First observed by Chen et. al., invadopodia are protrusive invasive structures that have focalized matrix proteolytic activity $(26,27)$. Invadopodia utilize many of the same regulatory and actin-nucleating proteins observed in lamellipodia. However, invadopodia also require the components needed to form microtubules and secrete matrix metalloproteases to facilitate ECM degradation. This collective activity allows resulting membrane protrusions to penetrate the basement membrane in order to remodel ECM (28-30). Invadopodia are complex structures comprising of a central Arp 2/3-generated F-actin core. Key actin polymerization regulators, including Arp 2/3, cortactin, Tks 5 and $\mathrm{N}$-WASp localize to the core region and are required for invadopodia formation (30-34).

Invadopodia are produced following stimulation by extracellular cues, where Ras family GTPases, such as CDC42/CDC42 GEF (Fgd1), non-receptor and receptor tyrosine kinases (RTKs) are activated (18,21,32,35-38). Src-phosphorylated Tsk5 binds and recruits the adaptor proteins Nck, cortactin and N-WASp to pre-invadopodia sites $(34,39,40)$. WIP, Nck, and N-WASp bind Src or Abl/Arg tyrosine kinases, which in turn tyrosine-phosphorylate cortactin to create docking sites for multimeric protein complexes (see below for details) that amplify Arp 2/3-mediated actin branching and subsequent invadopodial membrane protrusion $(30,36,37,41)$. Additional actin binding proteins assist in modulating invadopodia activity. For example, the actin binding protein cofilin regulates invadopodia assembly/disassembly by inducing barbed-end generation, Arp2/3 filament debranching and actin turnover $(32,37,42)$. Microtubules and intermediate filaments extend into nascent invadopodia and are essential for 
elongation (43). Invadopodia also contain radial, unbranched, bundled actin filaments that are polymerized by formins, where filament ends are uncapped by Ena/Vasp proteins to facilitate direct actin filament elongation by allowing preferential monomer addition at the "plus" end near the invadopodia tip (43-46). Parallel actin filaments are bundled by the proteins fascin, $\alpha$-actinin and caldesmon to provide structural integrity to the actin core (43,47-49). The adhesion proteins talin and paxillin localize to invadopodia and mediate integrin-based contact with ECM, but oddly the adhesion proteins focal adhesion kinase (FAK) and vinculin are not found in invadopodia (49-51). On a cellular level, adhesion and ECM degradation are inversely but tightly regulated; where degradation of matrix at sites of adhesion is counterproductive to adhesionbased force generation $(16,18,52)$.

Focalized ECM degradation occurs at tips and branch points of elongated invadopodia $(16,18,52,53)$. Several proteinases localize to invadopodia that are responsible for facilitating ECM degradation. These include matrix metalloproteinases MT1-MMP (MMP14), MMP2, MMP9, ADAM-family sheddases, cathepsin, seprase, and urokinase type plasminogen activator (UPAR)) $(21,29,54-59)$. MT1-MMP is widely considered the major and essential zinc-dependent membrane-bound MMP in regulating and enabling invadopodia proteolytic activity (the last stage in functional invadopodia maturation), as manipulation of MT1-MMP expression or activity directly modulates matrix degradation and invasion (29,57,60-62). MT1-MMP localization to invadopodia can occur through clatherin-mediated endocytosis (63-66) or by exocytic membrane trafficking (67-69).

Although the majority of work imaging invadopodia has been performed in 2D settings, invadopodia are believed to exist in vivo, since invadopodia forming cancer cell lines are more invasive in xenografts models than lines that do not form invadopodia. Also, primary cells from several tumor types, including HNSCC, bladder cancer and glioblastomas form functional invadopodia in classic 2D gelatin matrix degradation assays (70-73). In addition, invadopodia-like structures have been imaged in physiological relevant in vivo and ex vivo microenvironments containing key proteins associated with invadopodia, including N-WASp, Tks5, cortactin and Arp 2/3 that result in proteolysis of 3D ECM (69,74-77). Finally, actin-rich protrusive degradative 
structures termed podosomes are related to invadopodia, but are found in nontransformed cell types where they are essential for homeostatic invasive processes conducted by osteoclasts, macrophages, smooth muscle and endothelial cells $(21,78)$.

\section{Cortactin: A node of actin network regulation.}

Wu and Parsons identified cortactin as a substrate of Src kinase that localizes to cortical F-actin over 20 years ago (79). Cortactin is a NPF that simultaneously activates Arp 2/3 actin polymerization and also serves to stabilize resultant branched actin networks in lamellipodia and invadopodia (80). Cortactin is overexpressed in several different cancer types, including HNSCC (30\% of cases), breast (15\% of cases), lung, and bladder. Notably, cortactin overexpression in these and other tumor types is primarily due to amplification of the cortactin (CTTN) gene on chromosome 11q13 (81-86), where cortactin gene amplification has emerged as a prognostic marker for poor outcome, corresponding with increased risk for tumor recurrence and lymph node metastasis (84,87-90).

Cortactin is a multi-domain, rod-shaped protein that folds back onto itself to form a "Iollipop" shaped structure containing a globular region produced via intramolecular interactions $(80,91)$. Cortactin consists of five domains: an amino-terminal acidic domain (NTA), an F-actin binding domain (FAB), a helical region, a proline-rich region (PRR), and a Src-homology 3 domain (SH3) (Figure 2) (92). The NTA region of cortactin binds Arp 2/3 via the highly conserved DDW amino acid sequence (amino acids 20-22) found in other NPF proteins (93-96) that directly activates in vitro Arp 2/3 nucleation activity (97). Arp $2 / 3$ activation by cortactin is enhanced through additional indirect mechanisms that include the recruitment of cortactin binding proteins to other cortactin domains $(41,98-100)$. Adjacent to the NTA region, the F-actin binding domain consists of six and a half 37 amino tandem repeats that bind directly bind F-actin $(79,101)$. F-actin binding is regulated by acetylation/deacetylation of lysine residues within the repeats region. The histone acetyltransferases pCAF/p300 and SIRT1 acetylate cortactin and prevent F-actin binding, whereas the histone deacetylase HDAC6 deacetylates cortactin to facilitate F-actin re-binding (102-104). Cortactin binding to F-actin results in increased cell migration and is necessary for invadopodia 
formation and function (104-107). The helical region contains a calpain cleavage site that results in impaired lamellipodia protrusion and increased migration when proteolyzed by calpain 2 (108). The PRR and SH3 domains constitute regions that are responsible for receiving upstream signaling inputs via phosphorylation and regulated interaction of $\mathrm{SH} 3$ binding partners (Table 1) that ultimately govern downstream actinmediated cellular processes $(21,80)$.

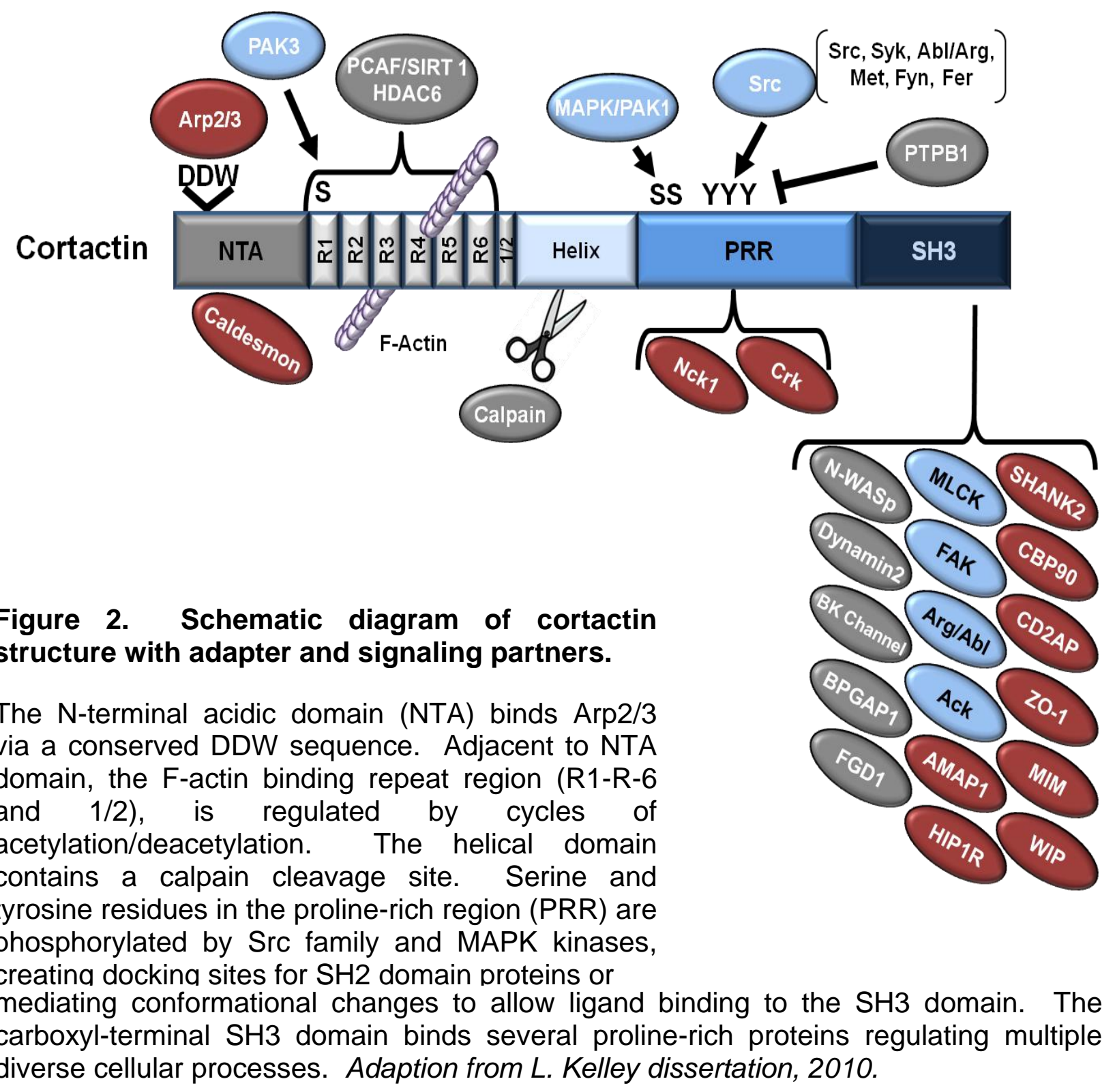


Table 1. Table of cortactin SH3 binding proteins.

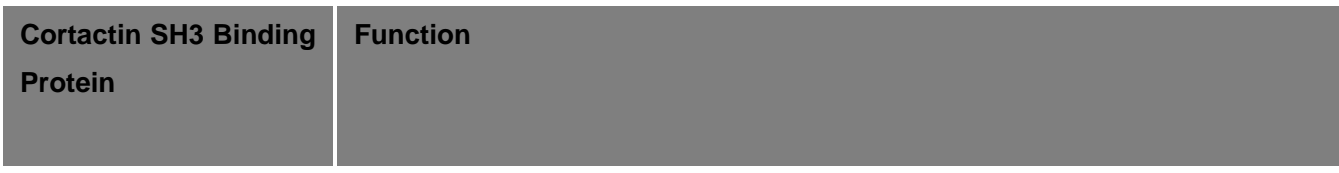

Abl/Arg

Phosphorylation of Y421, Y470, Y486 regulating dorsal waves, lamellipodia and invadopodia

CBP90

$\mathrm{ZO}-1$

A "brain specific" protein that bind cortactin at synaptosomal membrane, function unknown

Tight junction adaptor, binds cortactin regulating cell adhesion and spreading

RhoA-GAP, facilitates cortactin translocation to cell periphery increasing cell migration

BPGAP1

HIP1R

Caps barbed ends inhibiting actin polymerization mediating clatherin-mediated endocytosis

\begin{tabular}{l|l}
\hline BK channels & large conductance calcium- and voltage-activated potassium ion channels, \\
cortactin mediates Src regulation of BK channel function
\end{tabular}

ASAP1/AMAP1

Recycling endosomes focal adhesions, invadopodia podosomes/ ARF6 GAP

\begin{tabular}{l|l} 
N-WASp & $\begin{array}{l}\text { Neucleating promoting factor, enhances cortactin-based Arp2/3 nucleation } \\
\text { activity }\end{array}$ \\
&
\end{tabular}

Dynamin 2

GTPase, cortactin links dynamin2 and actin filament facilitating dynamin2 remodeling of actin filaments, membrane trafficking

CortBP1/SHANK

FGD1

Scaffold protein in postsynaptic sites, binds cortactin modulating actin remodeling

CDC42-GEF, enhances cortactin mediated Arp2/3 actin polymerization

\section{Reference}

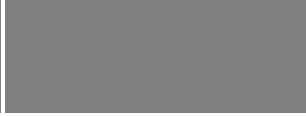

$(110,111,310)$

(377)

(378-380)

$(381,382)$

(383)

$(384,385)$

$(32,37,100,121,391$ -

393)

(277,394-397)

(398-400)

$(401,402)$ 


\begin{tabular}{|c|c|c|}
\hline WIP & $\begin{array}{l}\text { Forms complex with N-WASp and cortactin enhancing Arp2/3 mediated actin } \\
\text { polymerization }\end{array}$ & $(98,100,403)$ \\
\hline $\begin{array}{l}\text { Myosin Light Chain } \\
\text { Kinase (MLCK) }\end{array}$ & $\begin{array}{l}\text { Cortactin/MLCK interaction enhances myosin light chain phosphorylation and } \\
\text { positively regulates endothelial cell barriers }\end{array}$ & $(404-407)$ \\
\hline $\begin{array}{l}\text { Missing in Metastasis } \\
\text { (MIM) }\end{array}$ & $\begin{array}{l}\text { Enhances cortactin mediated Arp } 2 / 3 \text { actin polymerization increasing cortactin } \\
\text { induce motility. Src-mediated phosphorylation of cortactin is inhibited by MIM, } \\
\text { enhancing cilia formation }\end{array}$ & $(408-410)$ \\
\hline CD2AP & $\begin{array}{l}\text { Cortactin binds/recruits CD2AP to lamellipodia, CD2AP recruits barbed end } \\
\text { capping protein (CP) to lamellipodia. CD2AP binds cortactin and EGFR } \\
\text { regulating EGFR endocytosis }\end{array}$ & $(411,412)$ \\
\hline ACK & $\begin{array}{l}\text { ACK1 phosphorylates cortactin; ACK1/cortactin interaction mediates EGFR } \\
\text { internalization }\end{array}$ & (413) \\
\hline FAK & $\begin{array}{l}\text { Non-receptor tyrosine kinase, FAK/Src complex binds and phosphorylates } \\
\text { cortactin stimulating focal adhesion turnover and cell motility and regulates } \\
\text { irradiation induced JAK activation. }\end{array}$ & (133-135) \\
\hline
\end{tabular}

The cortactin PRR contains three key tyrosine residues (Y421, Y470, and Y486 in humans) that are phosphorylated by the Src family kinases Src, Fyn, Syk, and Fer, as well as the Abl family kinases Abl and Arg (109-113). Phosphorylation at these residues creating binding sites for the Src homology 2 (SH2) domain containing adaptors proteins Nck1 and Crk, as well as mediating Abl family kinase binding $(100,110,111,114)$. In the case of Nck1, the Nck1 SH2 domain binds pY421 and pY466, and recruits the Arp 2/3 NPF, N-WASp; N-WASp PRR domain binds cortacin $\mathrm{SH} 3$ domain creating a ternary complex that amplifies cortactin-based Arp2/3 nucleation activity indirectly through promoting N-WASp activation $(41,100)$. The net result of cortactin tyrosine phosphorylation is increased invasion and metastasis by enhancing lamellipodia persistence, dorsal wave formation and invadopodia ECM degradation activity $(37,41,110,111,115-119)$. Protein tyrosine phosphatase 1B (PTP1B) interacts 
with and dephosphorylates cortactin, consequently reducing cortactin-mediated invasion and metastasis (120).

Extracellular signal-regulated kinases Erk 1/2 (MAPK 3/1), are two related serine/threonine kinases that phosphorylate S405 and S418 in the cortactin PPR. Erk phosphorylation causes a characteristic shift in the $\mathrm{Mr}$ of cortactin from 80 to $85 \mathrm{kDa}$ in SDS-PAGE due to conformational alterations rather then simple addition of phosphate moieties (121-123). The close proximity of the targeted serine and tyrosine phosphorylation sites (especially S418 and Y421) has led to proposals where interplay between these two different phosphorylation events function in tandem to regulate cortactin signaling inputs. Work by Martinez-Quilles et al. (2004) with various tyrosine and serine cortactin phosphorylation mutants in in vitro biochemical assays indicated that serine phosphorylation by Erk 1/2 enhanced cortactin $\mathrm{SH} 3$ domain binding to the $\mathrm{N}$ WASp, PRR, resulting in N-WASp activation and Arp 2/3 activation apart from the tyrosine phosphorylation Nck1/N-WASp mechanism described above. Phosphorylation by Src led to decreased N-WASp binding and reduced Arp 2/3 actin polymerization. This group hypothesized that phosphorylation of cortactin serves as an on/off switch, where serine phosphorylation disrupts the intra-molecular interactions holding cortactin in a globular form to allow proline-rich proteins (e.g. N-WASp) to bind the exposed SH3 domain and become activated (the so-called "active" cortactin form). Conversely, tyrosine phosphorylation was proposed to promote the closed conformation by permitting re-folding of cortactin into the globular "inactive" conformation. While the precise mechanism of how Src-based tyrosine phosphorylation promotes cortactin inactivation was unclear, it was proposed that the modes of the serine/tyrosine switch function independently (121). Our lab (Study 3) demonstrated through the use of phosphospecific antibodies against cortactin Y421 and S418 that phosphorylation of serine and tyrosine residues occur simultaneously in cells, in contrast to these earlier findings $(123,124)$. Collectively these results suggest that cortactin tyrosine phosphorylation does not confer a closed "inactive" conformation proposed by the "serine-tyrosine switch". 
In addition to Src-, Abl- and Erk-family kinases, cortactin is also targeted by a variety of other kinases. The serine/threonine kinase p21-associated kinase (PAK1) phosphorylates S405/S418 following activation of Rac1 to regulate N-WASp-mediated vesicle motility (125). Phosphorylation of cortactin $\mathrm{S} 113$ by PAK3 in the first tandem repeat regulates cortactin binding to F-actin (126). Protein kinase D (PKD) targets cortactin at S298/S348, inhibiting WAVE2/Arp2/3 mediated actin polymerization (127129). In addition to these examples, there are 15 additional cortactin phosphorylation sites identified by mass spectroscopy that currently have unknown functions (130). Moreover, integrin-mediated FAK modulation of cortactin activity has divergent effects in Src-dependent and Src-independent manner. The FAK/Src complex binds and phosphorylates cortactin on $\mathrm{Y} 421, \mathrm{Y} 470$, and $\mathrm{Y} 486$ increasing focal adhesion turnover and cell motility (131-134). Integrin engagement also confers radiation resistance via FAK/cortactin downregulation of JNK in a Src-independent manner (135).

The carboxyl-terminal cortactin SH3 domain binds a multitude of different proteins (Table 1). These binding partners mediate cortactin regulation of various actin-based processes including cellular signaling (Fgd1, BPGAP1, Abl family kinases), actin polymerization (N-WASp, WASp-interacting protein (WIP)), membrane deformation (missing in metastatis, (MIM)), cell-cell and cell-substrate adhesion (ZO1 and FAK), endocytic and exocytic membrane trafficking (ACK1, HIP1R, AMAP, dynamin2, CortBP1/SHANK2, CD2AP), and actomyosin-based contraction (myosin light chain kinase (MLCK) $(80,136-138)$. These processes are collectively involved in cell migration and utilized in the organization invadopodia assembly. Several studies using deletion mutants have clearly determined that the cortactin $\mathrm{SH} 3$ domain is essential for proper invadopodia formation and function in cancer cell lines $(37,125,139)$. These results suggest cortactin creates a nexus for invadopodia regulation and subsequently serves as a driver of cancer cell invasion.

\section{EGFR: Signaling and significance in HNSCC.}

Epidermal growth factor receptor (EGFR, HER1, ErbB1) is a transmembrane tyrosine kinase that is a member of the ErbB family. Other members include ErbB2 (HER2), ErbB3, and ErbB4 (140). EGFR contains an N-terminal extracellular ligand binding 
region (domains I-IV), a hydrophobic transmembrane region, and a C-terminal cytoplasmic consisting of tyrosine kinase and autophosphorylation regions that propagate "outside-in" signaling (Figure 3) $(140,141)$. There are thirteen distinct ligands that can bind one or more ErbB receptors, adding to the complexity of ErbB signaling $(142,143)$. ErbB ligands are produced in the "pro" form and then transported to the plasma membrane, where they are inserted into the membrane or are exocytosed. In HNSCC, the sheddases ADAMS 10 or 17 cleave the pro-ligand to create an autocrine or paracrine signal $(144,145)$. Alternatively, the uncleaved EGFR ligand heparin binding-epidermal growth factor (HB-EGF) can bind adjacent cells to generate a juxtacrine signal (146). Epidermal growth factor (EGF), transforming growth factor $\alpha$ (TGFa) and amphiregullin (AREG) are common ErbB ligands that preferentially bind EGFR and are overexpressed in HNSCC, although other EGFR ligands also play lesser roles in HNSCC progression $(143,147,148)$.

Following ligand binding, ErbB receptors undergo a conformational change exposing a "dimerization arm" in domain II of the receptor to permit hetero- or homo-dimerization with a second ErbB receptor. Dimerization activates the tyrosine kinase domain, facilitating cross autophosphorylation of ten tyrosine sites of dimerized ErbB receptor cytoplasmic tail. The phosphorylation pattern varies depending on the ErbB/ligand combination $(149,150)$. Phosphorylated tyrosines in the EGFR cytoplasmic region bind $\mathrm{SH} 2$ domain-containing scaffold proteins (e.g. Shc, Crk, and Grb2) as well as downstream signaling proteins (e.g. STAT, PLCY, and Cbl). EGFR activation regulates several critical signaling pathways, mainly JAK2/STAT, RAS/Raf/MAPK, and PLCY/PI3K/Akt to govern cellular processes that include proliferation, survival, and motility (Figure 3) (151-155). EGFR signal attenuation occurs via clatherin-coated mediated endocytosis, with the targeting of EGFR-containing endosomes for surface recycling, degradation, or nuclear localization based on affinity of ligand and phosphorylation status. ErbB receptors with weakly bound ligands (e.g.; EGFR/TGFa) are recycled from early endosomes back to cell surface, whereas highly phosphorylated ErbB receptors are targeted for lysosomal degradation via late endosomes. Termination of EGFR signaling is assured through $\mathrm{SH} 2$ domain-mediated binding of the E3 ubiquitinase $\mathrm{Cbl}$ to phosphorylated tyrosine 1045 on EGFR, leading to ubiquitination 
of EGFR to target it for proteosome-mediated degradation (156-159). EGFR is also targeted to the nucleus through importin $\beta$ retrograde transport $(160,161)$, where it regulates cell proliferation and DNA repair. Nuclear EGFR is also an indicator of poor prognosis in cancer patients $(162,163)$.

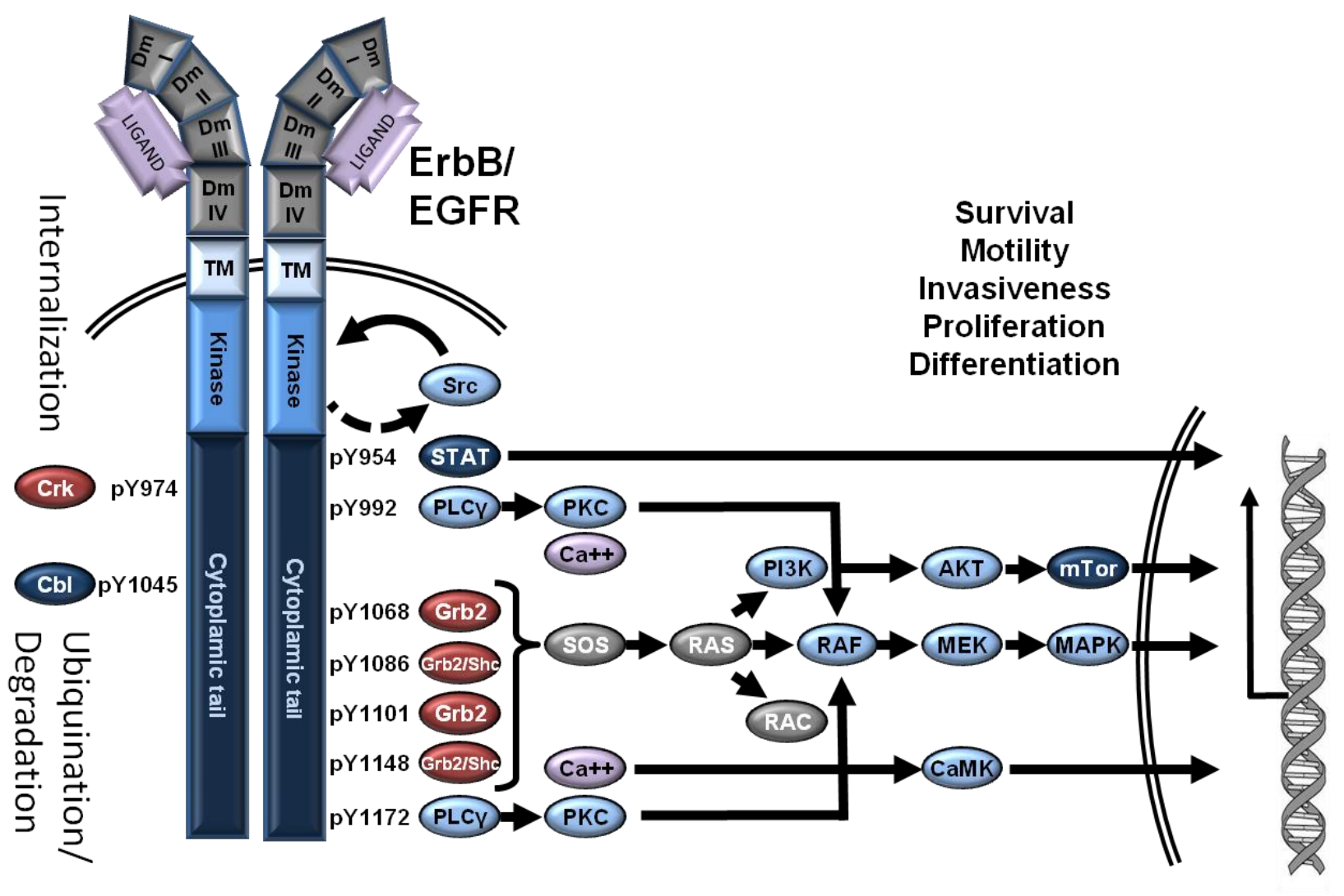

Figure 3. Schematic diagram of EGFR and conical MAPK signaling pathways.

Summary of EGFR-based signal transduction. EGFR extracellular region (domains (dm) I-IV) binds ligand, dimerizes and cross-phosphorylates a second ErbB receptor. Activated EGFR triggers three MAPK pathways: JAK/Stat, PI3K/Akt/mTOR, and RAS/RAF/Erk1/2 signaling cascades regulating transcription of genes that modulate cellular processes that induce tumorgenesis. Clatherin-mediated EGFR internalization is regulated by adaptor protein Crk. E3 ubiquitin ligase Cbl targets EGFR for degradation.

In addition to elevated proliferation and survival, activated EGFR regulates cell motility and invasion in HNSCC and other cancer types. EGFR activation stimulates activation of $\mathrm{Src}$ and Abl family tyrosine kinases that in turn govern the downstream production of 
lamellipodia and invadopodia $(36,37,100,111,115,116,164-168)$. Invasion and motility in HNSCC are directly inhibited with the small molecule EGFR targeting drug gefitinib $(115,169,170)$. EGFR activation in HNSCC also enhances MMP9 expression and activation $(171,172)$. These studies suggest a correlation between EGFR activity and pro-metastatic behavior in HNSCC.

The majority of stage III and IV HNSCC patients develop locoregional reoccurrences and/or distant metastasis $(4,141)$. EGFR overexpression occurs in $\geq 90 \%$ of all HPVnegative HNSCC, where it correlates with radiation resistant tumors and locoregional failures $(170,173)$. Up-regulation of EGFR signaling in HNSCC is further enhanced by the expression of EGFR ligands. EGF is overexpressed in $65 \%$ of cases, TGFa in $90 \%$ and AREG in $45 \%((170,174,175)$. Recent work indicates that EGFR expression and activation levels are independent predictors of poor prognosis for HNSCC patients (176). The observed high rate of elevated EGFR activation is likely not due to activating point mutations, which rarely arise in HNSCC. However, a truncated version of EGFR (EGFRVIII) with deletion of exons 2-7 occurs in $17-42 \%$ of the HNSCC cases (177179). Exon 2-7 deletion removes the extracellular domain, rendering it constitutively active and resistant to monoclonal antibody-based therapeutic inhibition $(177,180,181)$. In addition to HNSCC, several other solid tumor types overexpress EGFR, including non-small cell lung carcinoma (NSCLC, 62\%), breast cancer (50-70\%) and glioblastomas (>50\%) (182-184).

Multiple therapeutic EGFR inhibitors exist today designed to disrupt or ablate EGFR dimerization or kinase activity. Prominent examples include cetuximab, panitumumab, gefitinib, vandetanib, erlotinib and lapatinib. EGFR targeted therapy can be subdivided into two categories; monoclonal antibodies or small molecule inhibitors $(181,185)$. Cetuximab is a monoclonal antibody that has been approved for concomitant therapy with radiation or platinum-based chemotherapy for recurrent or metastatic HNSCC based on the increased response rate in phase III trial $(8,141,186,187)$. Gefitinib is a small molecular EGFR inhibitor approved for patient treatment. However, combination therapy of gefitinib and methotrexate in phase III trial of chemoresistant recurrent/metastatic HNSCC patients did not improve overall response rate 
$(181,188,189)$. The high percentage of different solid tumor types combined with sporadic success of targeted therapeutic approaches continues to keep EGFR in the spotlight as an important molecular target in head and neck oncology.

\section{Erk1/2: Effector proteins for EGFR signaling.}

Extracellular signal-regulated kinase 1 and 2 (Erk 1/2, MAPK 3/1) were the first discovered members of the mitogen-activated protein kinases (MAPK) kinase family and are ubiquitously expressed. Erk $1 / 2$ and other MAPK members including c-Jun Nterminal kinase (JNK), p38 MAPK, and Erk5 are serine/threonine kinases activated by various extracellular cues $(190,191)$. Erk $1 / 2$ proteins are comprised of an N-terminal domain that facilitates binding to ATP and a C-terminal catalytic domain containing a conserved "TXY" amino acid sequence targeted by upstream kinases. A channel generated between the two terminal domains creates a binding pocket for Erk 1/2 substrates (192-194).

Erk $1 / 2$ has approximately 180 substrates that impact proliferation, differentiation, cellular survival/apoptosis, differentiation, motility, and invasion $(195,196)$. Given the complexity of Erk $1 / 2$ signaling network, Erk activity is tightly regulated in a spatial and temporal manner. The subcellular localization of Erk proteins is determined by several factors, including binding of Erk 1/2 to the tethering proteins VDAC, SEF, or LSP1 at mitochondria, Golgi, or the plasma membrane, respectively (195). Erk 1/2 compartmentalization is also influenced by the duration and strength of upstream EGFR signals, where strongly EGF-activated EGFR generates a transient cytoplasmic signal, while weakly bound EGFR ligands initiate sustained Erk activation and relocation of Erk kinases to the nucleus $(140,195)$.

The mechanism of EGFR-mediated Erk 1/2 activation is well described. Following EGFR activation, Src homology 2 containing (Shc) protein binds to phosphorylated tyrosines on the EGFR cytoplasmic domain. This in turn recruits growth factor bound protein 2 (Grb2) and son of sevenless (SOS), a RAS guanine nucleotide exchange factor (GEF). SOS activates one of three different RAS isoforms (H-RAS, N-RAS, or KRAS) by converting GDP-bound RAS to GTP-RAS (197). RAF (A-RAF, B-RAF, or C- 
RAF) are a class serine/theronine kinases that localize to the plasma membrane and are activated by GTP-RAS. Activated RAF in turn phosphorylates mitogen-activated protein kinase kinase (MEK or MAP2K), resulting in MEK activation $(191,195,198,199)$. The RAF/MEK complex is stabilized by the scaffold protein kinase suppressor of Ras 1 (KSR1), which also recruits Erk 1/2 $(200,201)$. Activated MEK in turn phosphorylates Erk 1/2 on T183 and T185 to generate an active kinase $(202,203)$. Once activated, the protein complex disassociates and releases activate Erk 1/2, which either localizes to the nucleus to regulate transcription, or to specific cytoplasmic compartments to regulate other cellular processes (detailed in Figure 4) $(191,195,198,199,204)$.

Since Erk $1 / 2$ controls an array of cellular processes, its activation cycle is tightly regulated. Erk $1 / 2$ is inactivated by MAPK phosphatases (PP2A and PP2C) (205) and dual specific phosphatase (DUSP) (206). Erk 1/2 itself also regulates several of the factors involved in the RAS activation pathway. SOS phosphorylation by Erk 1/2 disrupts the SOS/Grb2 interaction (207), Erk1/2-mediated phosphorylation of EGFR Y669 increases EGFR phosphoryaltion and inhibits EGFR degradation, enhancing EGFR activity (208) RAF and MEK are also inhibited by Erk 1/2 phosphorylation $(209,209,210)$. In addition, DUSP transcription is induced by Erk $1 / 2$ phosphorylation of transcription factor Ets (211). Collectively, Erk 1/2 activation regulates several feedback loops that converge at multiple levels to provide fine tuning of the Erk 1/2 signaling cascade $(191,212,213)$.

In addition to EGFR, Erk $1 / 2$ activation is facilitated by integrin signaling in a noncanonical manner. Integrin-mediated activation of FAK induces activation of Src, which initiates Ras activation to increase Erk 1/2 activity (214-216). Erk activation stimulated by integrin engagement imparts multiple effects that manifest in increased invasion and metastasis in many tumor types. HNSCC cells plated on fibronectin enhances Erk 1/2 activation (217). FAK activity induced by $\alpha \mathrm{V} \beta 3$ integrin engagement enhances Erk and Akt activation, increasing the aggressiveness of chronic myologenous leukemia $(\mathrm{CML})$ (218). The aforementioned Erk1/2-mediated serine phosphorylation of cortactin regulates invadopodia proteolytic activity in HNSCC and melanoma cell lines $(115,123,139)$ and lamellipodia formation in breast cancer lines $(123)$. 


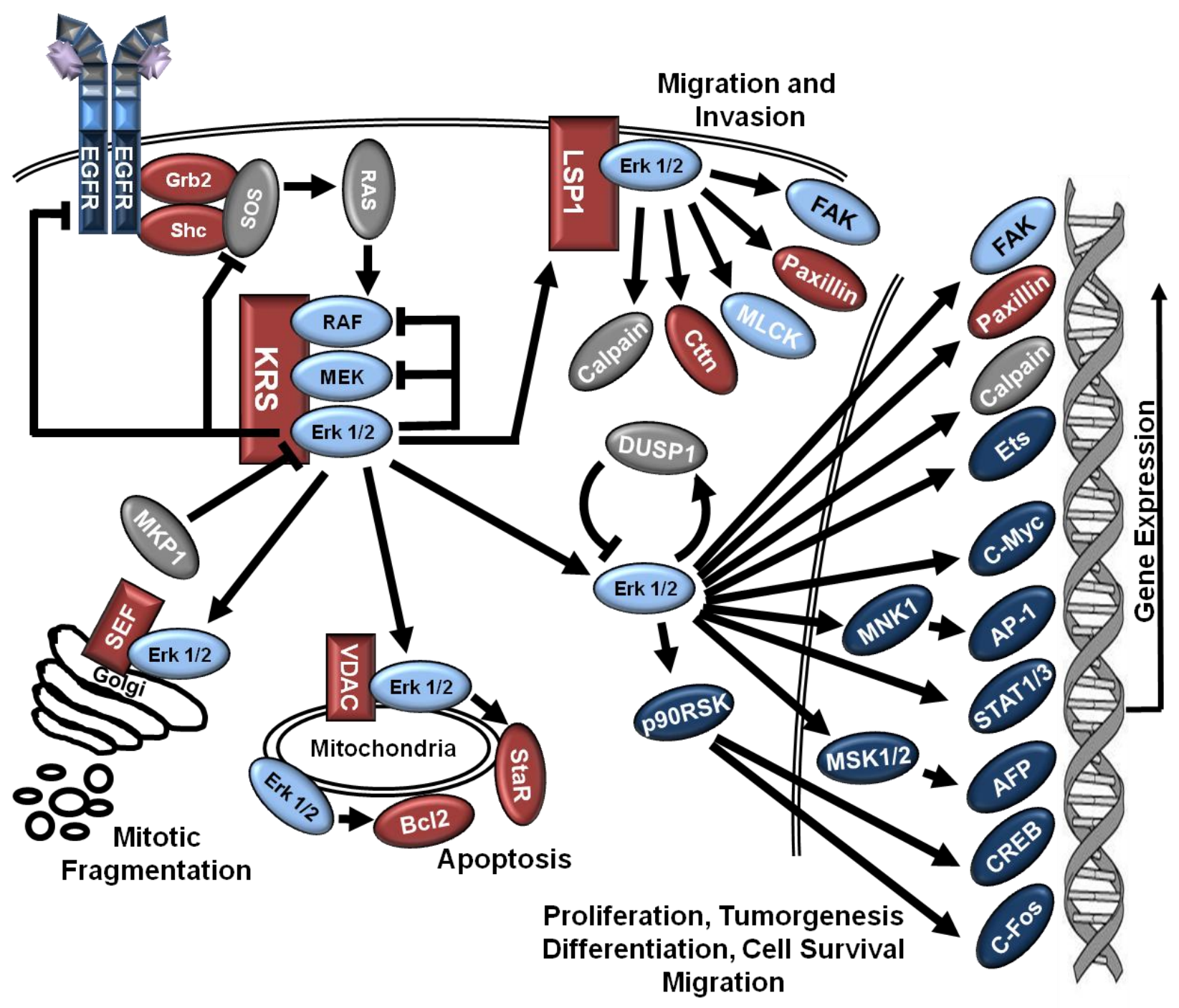

Figure 4. Erk1/2 signaling cascade and regulation of cellular processes.

Summary of the RAS-RAF-MEK-ERK $1 / 2$ activation cascade. Following EGFR activation, the Grb2/Shc adapter complex activates SOS RAS-GEF activity, stimulating RAS to activate RAF. RAF/MEK/Erk1/2 form a complex and signaling cascade, RAF activating MEK, MEK activating Erk 1/2. Erk 1/2 regulates several cellular processes in the nucleus via several transcription factors to modulate proliferation, differentiation, cell survival, and migration. Cytoplasmic Erk 1/2 regulation of migration and invasion, apoptosis, and Golgi fractionation is controlled in part by cytoplasmic location. Erk 1/2 activity is tightly regulated via several negative feedback loops mediated by Erk 1/2.

In HNSCC patient samples with EGFR and TGFa overexpression, Erk activation correlates with more aggressive recurrent disease and regional lymph node metastasis (219). Hyperactivation of Erk $1 / 2$ can occur by activating mutations in kinases and 
regulators upstream of Erk activation cascade. These include gain of function mutations in RAS (typically K-RAS) that stimulate cell proliferation in $30 \%$ of all cancer and $90 \%$ of pancreatic adenocarcinoma patients. Generally, mutations in the P loop (G12) or catalytic region (Q61) of K-RAS renders K-RAS resistant to GAP inactivation. Downstream from RAS, B-RAF is commonly mutated in melanoma (39\%) and papillary thyroid (38\%) carcinomas. This results in hyperactivation of the closely related C-RAF as described above. However, RAS and RAF-activating mutations are seldom seen in HNSCC, suggesting Erk activation is more likely due to integrin engagement, EGFR activation or other factors (220).

Given the central importance of Erk activity in human cancers, several pharmacological agents targeting the Erk $1 / 2$ signaling pathway have been generated to date. Selumetinib (AZD6244, utilized in Study 3) inhibits Erk 1/2 via inhibition of MEK by functioning as a non-competitive ATP-binding inhibitor. While well tolerated in early phase I clinical trials, no significant benefit in progression free survival was observed in colorectal or NSCLC patients treated with selumetinib in phase II trials (220-223). Other MEK inhibitors in clinical or preclinical trials are Cl 1040 (PD184-352) and U0126, which demonstrate some efficacy in preclinical work, but to date but have not been extensively tested in the clinic (220). Sorafenib, an inhibitor designed to target all major RAF isoforms, has shown some efficacy in a phase II trial of hepatocellular carcinoma, but did not increase the overall survival of patients in a phase III trial $(220,224,225)$. The overall ineffectiveness of MEK and RAF inhibitors in the clinic suggests that alternative MAPK pathways are likely compensating to overcome Erk 1/2 inhibition, or Erk is being activated by alternate mechanisms.

\section{Src: The first oncogene.}

Over a century ago, Peyton Rous discovered the first oncogene that caused cancer in chickens (226). Termed v-Src, the human homolog c-Src was discovered years later and was subsequently identified as a proto oncogene in human cancers (227). Src is one member of a kinase family that incorporates a number of related non-receptor tyrosine kinases including the closely homologous members Yes, and Fyn in epithelia. Src modulates several cellular processes, including cytoskeletal organization, cell-cell 
interactions, cell-matrix adhesion, EMT, migration and invasion $(228,229)$. Src has a modular molecular structure, consisting of an N-terminal Src homology 4 (SH4) domain that is myristolyated to allow membrane association (230-232). SH3 and $\mathrm{SH} 2$ domains are adjacent to the $\mathrm{SH} 4$ domain and function to mediate inactivating intramolecular interactions, as well as for binding to Src adapter and substrate proteins. The SH1 domain contains the catalytic region at the C-terminus (Figure 5) $(229,231,233,234)$.

Within the C-terminal tail is a critical tyrosine residue (Y527) phosphorylated by Cterminal Src kinase (CSK). Phosphorylated (p)Y527 binds the SH2 domain to form a stable interaction that maintains $\mathrm{Src}$ in an inactivate state $(235,236)$. Inactivation is also reinforced by $\mathrm{SH} 2 / \mathrm{SH} 1$ and $\mathrm{SH} 3 /$ linker region interactions (237). Src activation is accomplished through a multi-step process initiated by the binding of substrates and/or scaffolding proteins to the $\mathrm{SH} 2$ or $\mathrm{SH} 3$ domain to disrupt intramolecular interactions between the catalytic and linker regions. Activation is further achieved through the action of the tyrosine phosphatases; PTP1B, SHP 1/2, PTPa, which dephosphorylates $\mathrm{Src}$ pY527, fully releasing the C-terminal tail from the $\mathrm{SH} 2$ domain to allow "opening" of Src into the active conformation (238-241). Full activation is achieved by subsequent autophosphorylation of tyrosine 419 in the kinase domain (237). In cellular contexts, integrin engagement stimulates formation of FAK/Src or p130CAS (CAS)/Src complexes through Src SH2 domain binding, achieving similar opening of the kinase and subsequent Src activation (242). Alternatively, Src activation occurs downstream of G-coupled protein receptors (GCPR) or the receptor tyrosine kinases PDGFR, EGFR, FGFR, c-Met, and HER (165,243-250). Src phosphorylates and activates receptor tyrosine kinases (e.g.; EGFR) and FAK, creating positive feedback loops that enhance Src activity.

Src mediates cellular invasion and migration by phosphorylating several key components involved in these processes. Phosphorylation of paxillin, p130CAS, and FAK disrupt focal adhesions, enhancing adhesion turnover and subsequent migration (242,251-254). Src also hinders contractility by activation of p190RhoGAP (a Src substrate), inhibiting RhoA/Rho kinase/myosin light chain II (MLC II) mediated contraction (255-257). Alternately, Src induces RAS-activation to stimulate Erk 1/2 
activation and phosphorylation of myosin light chain kinase (MLCK)/MLC II, increasing contractility and disassembly of focal adhesions $(258,259)$.

Src phosphorylates several key proteins involved in invadopodia assembly, maturation, and disassembly. Src phosphorylates and regulates AMAP1 (Arf6 effector protein) and Tks $4 / 5$, both events that serve to modulate invadopodia actin polymerization and localization of proteins that initiate invadopodia assembly. AMAP1 binds and localizes CIN85 and Cbl to invadopodia, increasing ECM degradation. AMAP1 also binds cortactin and is required for driving breast cancer cell invasion (260-263). Following Src phosphorylation, Tks 4/5 activates NADPH oxidase to stimulate reactive oxygen species (ROS) production, creating a possible positive feedback ROS-induced activation of Src. Tsk 5 also recruits several key invadopodial proteins including cortactin and Nck responsible for regulating invadopodia function (34,39,40,264-268). In addition, Src mediates invadopodia maturation via regulation of MMP production and secretion by modulating PI3K, MAPK, and JAK/STAT activation (269-271). Src modulates vesicle trafficking through phosphorylation of endophilin A2, Cdc42-interacting protein (CIP4), and dynamin2. Src phosphorylation of CIP4 (Y471) inhibits CIP4 mediated endocytosis of MT1-MMP, which results in elevated surface expression of MT1-MMP, increasing invadopodia proteolytic activity (272). Src phosphorylation of Endophilin A2 tyrosine 315 functions in a similar manner, increasing invadopodia activity by inhibiting endophilin-induced MT-MMP endocytosis (273). In addition, dynamin requires Src phosphorylation at tyrosine sites $231 / 597$ to mediate clathrin-coated endocytosis of MT1-MMP (274-277).

Cortactin tyrosine phosphorylation is crucial for invadopodia maturation, where Src regulates cortactin phosphorylation directly or indirectly via Abl/Arg family kinases (37,41,110,111,115-119). How Src controls invadopodia disassembly is not as clear, but Src phosphorylation of paxillin Y31 and Y118 promotes invadopodia disassembly, providing at least one mechanism $(278,279)$. Calpain 2 and PTP1B function together to regulate invadopodia function, where calpain induces invadopodia turnover potentially via Pyk2, WASP, and talin cleavage (Figure 5) $(280,281)$. 


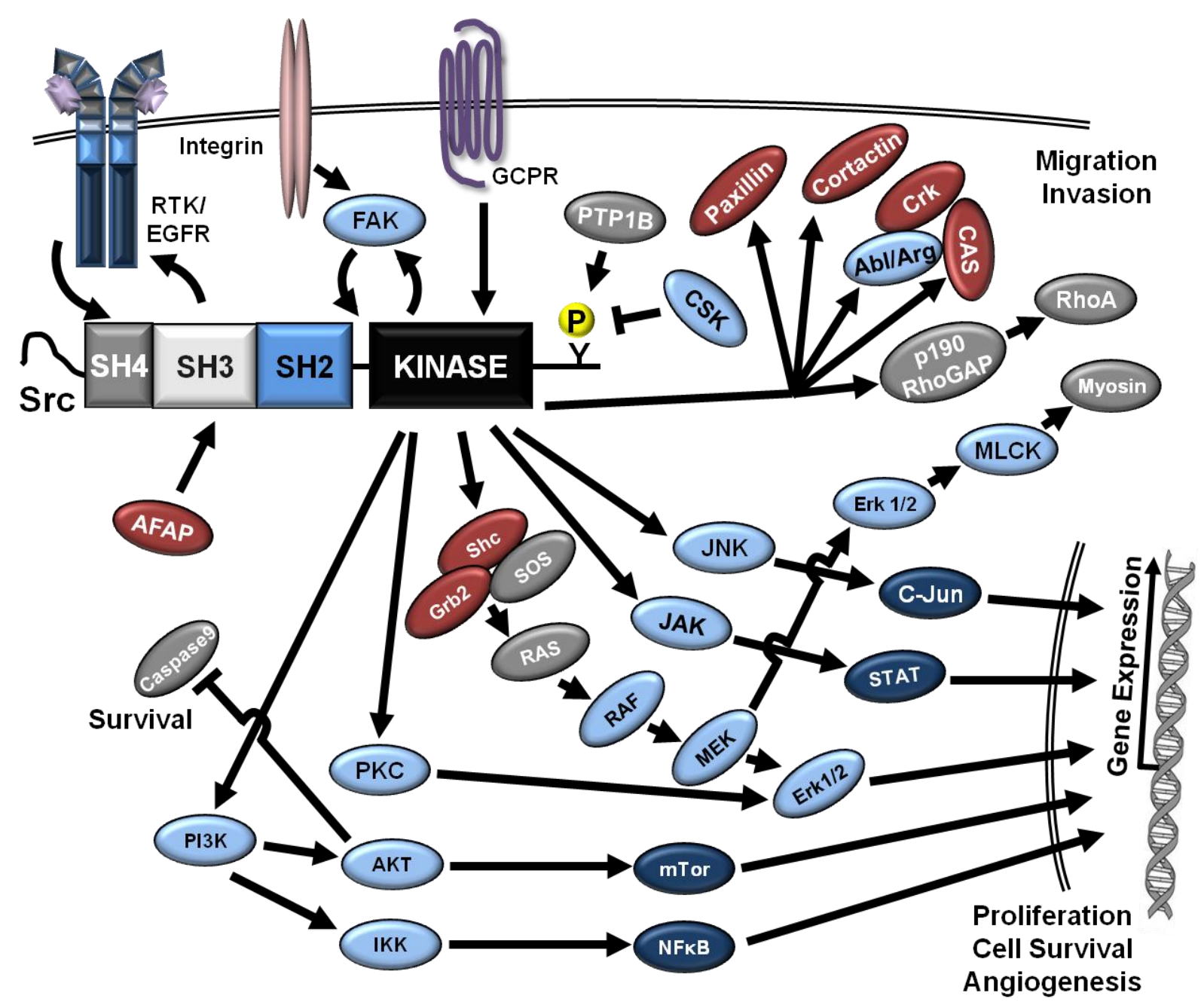

Figure 5. Schematic diagram of Src, activation and downstream signaling pathways.

Overview of Src-based signaling in invasion and proliferation. Src becomes activated as described in the main text, where it stimulates several signaling cascades including RAS/RAF/MEK/MAPK, JAK/STAT, and PI3K/Akt cascades regulating transcription, proliferation, cell survival and angiogensis. Src also phosphorylates several key components involved in contractile and mesenchymal-based migration and invasion.

Src overexpression frequently occurs in HNSCC, where its activation correlates with poor prognosis (282). Src activation can be enhanced via several mechanisms that occur in HNSCC and other cancer types, including downregulation of CSK expression, PTP1B overexpression, activation of receptor tyrosine kinases, or activation of integrins 
through ECM alteration (283). Importantly, ionizing radiation, which is frequently utilized as first line therapy in HNSCC and other cancers, stimulates Src activity due to the generation of reactive oxygen species (ROS) increasing the potential for triggering invasion and metastasis in radiation-resistant and recurrent tumors (284).

The inhibition of Src activity has become an important pursuit for molecular therapeutics since $\mathrm{Src}$ is a key regulator of many of processes utilized in tumorigenesis. Dasatinib, saracatinib, and bosutinib are three Src inhibitors undergoing phase I and II trials as single agents or combination therapy with conventional chemotherapeutics. These inhibitors all function in a similar manner by blocking ATP binding to the $\mathrm{SH} 1$ catalytic domain and have off-target effects, one of which includes serendipitous inhibition of Abl family kinases $(283,285)$. Dasatinib (BM354825) treated HNSCC cell lines display reduced activation of Akt and Erk 1/2, resulting in impaired Bcl-2 expression, growth inhibition and increased apoptosis $(286,287)$. In recent studies, dasatinib treatment inhibited invasion and metastasis of pancreatic cancer cell lines $(288,289)$. Preclinical trials with dasatinib-treated breast cancer cell lines showed reduced proliferation, migration and invasion (290-292). However, recent phase II trails with breast cancer demonstrated minimal added benefit with dasatinib treatment (293-295). Our laboratory (Study 2) and others have demonstrated that saracatinib (AZD0530) treatment inhibits Src and FAK activity in HNSCC cell lines, causing reduction in proliferation and migration in vitro and in vivo $(296,297)$. In several studies, saracatinib treatment inhibited pancreatic tumor xenograph growth and downstream phosphorylation of the Src substrates FAK, paxillin, and STATs $(298,299)$. Use of saracatinib in a phase II trial also demonstrated minimal efficacy in patients with advanced melanoma (300).

\section{Abl: A kinase with dichotomous roles.}

Abl (Abl1, c-Abl) and Arg (Abl2) are non-receptor tyrosine kinases with 90\% homology in the $\mathrm{N}$-terminal region but with extremely variable C-termini (<28\% homology) (301). The N-terminal domain, similar to Src, contains a capped region that may be posttranslationally modified by myristoylation, generating two isoforms (Abl1a and Abl1b) $(302,303)$. Adjacent to the capped region are highly conserved $\mathrm{SH} 3$ and $\mathrm{SH} 2$ domains (304). Attached by a linker region to the $\mathrm{SH} 2$ domain, a tyrosine kinase domain targets 
substrates with (L/I/V)-Y-X-X-P consensus sequence (305). Abl family kinases have over one hundred identified and potential substrates (306). Unlike other Src family kinases, Abl family kinases have a C-terminal region that consists of several proline-rich regions (Abl has four, Arg three) interspersed with three nuclear localization sequences (Abl only) (307,308). Abl and Arg diverge structurally in the C-terminal region, where Abl consists of a DNA binding region, a G-actin binding domain, and a F-actin binding region with an embedded nuclear export sequence (309-312). The Arg C-terminus includes two F-actin binding domains, a G-actin binding domain and a microtubule binding domain (Figure 6) $(313,314)$. These differences confer some of the divergent functions for Abl and Arg in the cell. Arg is solely a cytoplasmic protein that functions to regulate actin-based lamellipodia and invadopodia, but additionally bundles actin filaments and connects actin networks with microtubules. Abl shuttles between the cytoplasm, where it also regulates dynamic actin structures, and nucleus where it is key in governing transcription, cell cycle progression and DNA damage response $(315,316)$.

Abl family kinases, like Src, are tightly controlled by intramolecular interactions. Abl is held in an inactive conformation by binding of the myristolated tail within a hydrophobic pocket in the kinase domain $(302,303,317)$. Interactions between the SH3 and SH2 domains with the kinase domain also help hold $\mathrm{Abl}$ in an inactive state $(304,317)$. Abl becomes weakly activated by disruption of intramolecular interactions following binding of substrates/ adapter proteins to the $\mathrm{SH} 3$ and $\mathrm{SH} 2$ domains (302). To achieve full kinase activity, Src phosphorylation of Abl or Abl trans-phosphorylation of Y245 in the linker region and Y412 in the kinase domain activation loop is mandatory $(165,315,318$ 320). Additional Abl sites are phosphorylated that regulate protein stability (Y89 and Y261), kinase inhibition (Y272), activation (Y276), generation of potential SH2-binding sites (Y158, Y331, Y134, Y147, Y251, Y276), or are acetylated to control nuclear export (K730) (306).

Abl family kinases are activated by several extracellular signals, EGFR, PDGFR, insulin-like growth factor 1 receptor (IGFR), integrin signaling, bacterial invasion, genotoxic and oxidative stress (165,308,321-323). Upon activation via RTK or integrins, activated Abl family kinases localize to the cell periphery, where they regulate actin- 
based processes including lamellipodia protrusion and invadopodia maturation $(36,37,110,111,308,315,324,325)$. Abl enhances actin polymerization via phosphorylation of the NPFs WAVE2, N-WASp and cortactin. Abl also phosphorylates the adaptor protein Nck1 and the actin filament capping protein Ena, enhancing Enamediated actin filament elongation $(37,111,307,315,326,327)$.

Abl kinases directly and indirectly modulate cell migration and invasion. Arg phosphorylates p190RhoGAP, preventing binding to p120RasGAP and subsequent relocation of p190RhoGAP/p120RasGAP complex to cell periphery, thus preventing Rho GTPase activation reducing myosin-induced contractility $(328,328,329)$. PDGFR mediated Rac activation is modulated by Abl; although the mechanism has yet to be defined it is likely via Abl phosphorylation of SOS1 an RASGEF $(330,331)$. Ablmediated phosphorylation of CrkIl (Y221) and paxillin disrupts the Crk/CAS/Paxillin/FAK complex, resulting in disassembly of focal adhesions (308,332-337). Abl indirectly activates STAT3 to induce MMP1 transcription; Arg activates MT-MMP, MMP1, and MMP3 transcription in an undefined STAT3-independent manner. Additionally, Abl/Arg potentially mediate MT1-MMP endocytosis by directly binding and phosphorylating MT1-MMP $(324,325)$. Collectively these cellular processes confer pro-invasive and anti-invasive phenotypes dependent on the substrate and cellular localization of Abl or Arg.

Nuclear localization of Abl occurs in response to genotoxic or oxidative stress $(321,322,338,339)$. Ataxia telangiectasia mutated protein (ATM) activates Abl in response to DNA damage $(340,341)$. Activated Abl phosphorylates murine double minute 2 (MDM2) at tyrosine 394 an E3 ubiquitin ligase that targets p53 for degradation causing the disruption of the MDM2/p53 interaction protecting p53 from degradation (342-345). Abl stabilizes ATM/p53 complex facilitating ATM serine phosphorylation of p53 and subsequent accumulation of p53 in the nucleus regulating cell cycle arrest and/or apoptosis by transcription of p21 and CDK2 or BAX, BCL2, PUMA, and NOXA $(346,347)$. Abl initiates DNA repair mechanisms or induces apopotosis by phosphorylating and activating several key proteins including ATM, several RAD proteins, damaged DNA-binding (DDB 1/2), and DNA-activated protein kinase (DNAPK) 
(348-351). Abl also regulates cell cycle progression, where phosphorylation of RNA polymerase II induces transcription of $S$ phase genes and cell cycle progression from $S$ to G2-M $(342,352)$. Collectively, Abl is considered a pro-apoptotic regulator following nuclear localization and activation (Figure 6) $(316,353)$.

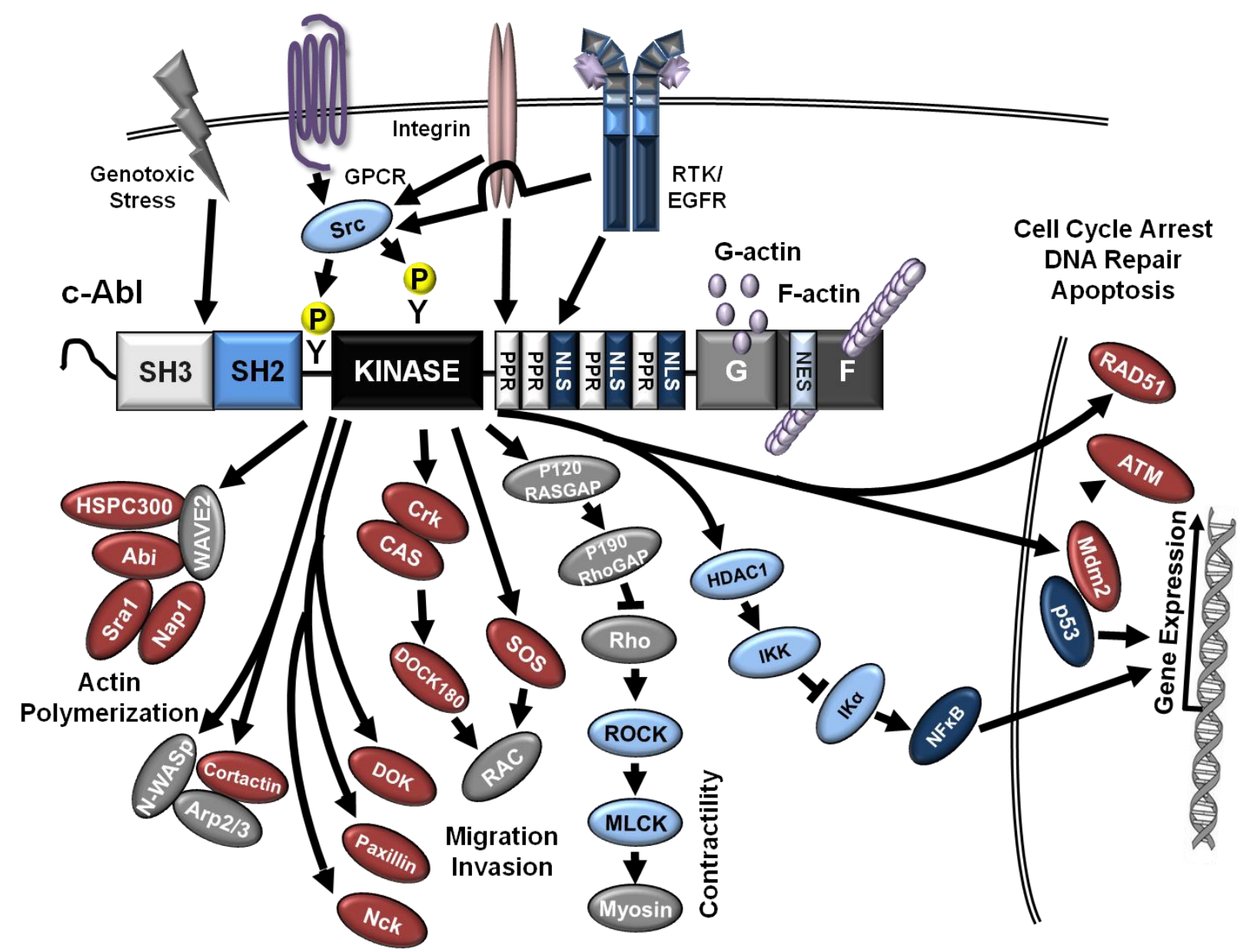

Figure 6. Schematic diagram of Abl structure, activation and downstream signaling pathways.

Abl a non-receptor tyrosine kinase becomes activated via external stimuli; phosphorylation and binding of $\mathrm{SH} 3 / \mathrm{SH} 2$ binding partners containing proline-rich regions or phosphorylated tyrosines. Activated Abl, in the cytoplasm, mediates processes utilized for motility and invasion (detailed in text). In the nucleus, Abl regulates transcription directly or indirectly via transcription factors controling cell cycle arrest, DNA repair, and apoptosis. 
Abl activation is attenuated by several mechanisms including de-phosphorylation by tyrosine phosphatases including PTPN1 or PTP-PEST (354). Phosphatidylinositol-4,5bisphosphate $\left(\mathrm{PIP}_{2}\right)$ binds $\mathrm{Abl}$ preventing substrate binding; the PIP2 inhibitory interaction is relieved by phospholipase $\mathrm{C}$ (PLC) hydrolysis of $\mathrm{PIP}_{2}(164,355)$. Caspasecleavage of $\mathrm{Abl}$ at Argine 565 or ubiquitinatation and proteosomal degradation downregulate Abl signaling (338,356-358).

Abl has long been considered an oncogene in the context of the Philadelphia chromosome BCR-Abl gene product. BCR-Abl an oncogenic fusion of the Abl kinase domain with the breakpoint cluster region (BCR), giving rise to a constitutively active $\mathrm{Abl}$ kinase by the removal of first exon and subsequent loss of inhibitory intramolecular interactions within Abl. BCR-Abl is associated with the onset and progression of chronic myelogenous leukemia (CML) $(359,360)$. The role of $\mathrm{Abl}$ in solid tumors is not as well defined, as recent work described below indicates that Abl family kinases function in a context dependent manner, possessing pro- or anti-oncogenic functions in cancer.

Research with a panel of breast cancer cell lines suggested that kinase activation and not expression of Abl family kinases correlates with breast cancer invasiveness $(361,362)$. Mader et.al. and others have shown the loss of invadopodia proteolytic activity with imatinib-treated breast cancer and melanoma cell lines, leads to the proposal of an EGFR/Src/Arg/cortactin signaling pathway responsible for mediating invadopodia regulation $(36,324)$. The imatinib-mediated inhibition of ECM degradation in breast cancer lines can be attributed in part to regulation of MMP 1, 3, and MT1-MMP transcription and secretion regulated by Abl and Arg $(324,325)$. In NSCLC, inhibition of Abl with FUS1 or pharmacological agents reduced anchorage-independent growth, while Abl phosphorylation of Crk correlates with tumor cell aggressiveness (363). Collectively these studies suggest a pro-oncogenic role for Abl family kinases.

Alternately, reduction of $\mathrm{Abl}$ expression in HNSCC is associated with a poor patient survival (364). Inhibition of Abl expression or activity reduces invadopodia proteolytic function and invasion (115). Similar results were observed in work on breast cancer lines by other groups, where Abl mediates EphrinB1/EphR4 inhibition of cell migration and suppresses TGF $\beta$-induced epithelial-to-mesenchymal transition (EMT) (365). 
Additionally, Frasca et al. demonstrated that HGF-simulated thyroid cancer cell lines treated with imatinib had elevated Erk and Akt activity, increased motility and an EMTlike phenotype following long-term drug exposure (366). These results indicate an antioncogenic role for Abl.

Imatinib mesylate (Gleevac, STI571) is a competitive ATP inhibitor and first line therapy for CML patients. This compound is extremely effective, demonstrating a $90 \%$ overall response rate in patients following initial treatment (367). Second line therapies that target Abl kinase in BCR-Abl containing malignancies include nilotinib, dasatinib, bafetinib (INNO-406) and decipere (DCC-2036). These compounds have been developed to treat imatinib-refractory patients and are currently in pre-clinical, phase I and II trials (368). Nilotinib and dastinib have a $50 \%$ complete response rate in phase II trials with imatinib-resistant CML patients (368). The efficacy of Abl inhibitors in CML and gastrointestinal stromal tumor (GIST) patients supports a pro-oncogenic role, advocating the use of imatinib for patients with solid tumors (369). Even though preclinical studies have demonstrated efficacy with imatinib in NSCLC and breast cancer cell lines, clinical phase I and II trials have shown no therapeutic benefits. In fact, disease progression was observed in a phase II prostate cancer trial. Furthermore, a combined HNSCC/NSCLC phase II trial was terminated early due to detrimental effects in patients treated in the imabinib-containing arm (370-374,374-376). The pro- and antioncogenic impact of Abl inhibition in solid tumors necessitates further investigation to determine the underlying molecular determinates responsible for the opposing oncogenic roles of Abl kinases in human cancers.

As detailed in this review, EGFR, Erk 1/2, Src, and Abl have complex signaling cascades dependent upon extracellular cues, upstream activators, cellular location, and downstream effectors. In pre-clinical studies, EGFR, Erk 1/2, Src, and Abl have been shown to be crucial for tumorigenesis in several cancer types, including breast and HNSCC. Unfortunately, the various pharmacological agents targeting these kinases (EGFR, Src, Abl) or up-stream activators (RAS, RAF for Erk1/2) typically demonstrate negligible therapeutic benefit; in fact detrimental effects have been reported in pancreatic, NSCLC and HNSCC cancer patients. Clarification and increased 
understanding of the spatial and temporal molecular signaling of these key proteins is essential for improved therapy utilizing these pharmacological agents. The specific goal of this dissertation is to clarify the regulation of key signaling molecules that modulate HNSCC invasion and metastasis.

In Study 1, we elucidated the regulatory hierarchy of several proteins critical for invasion of HNSCC and breast cancer cell lines. In particular, we focused on Abl, a protein that has opposing roles in tumorigenesis. Our results demonstrate a divergent role for Abl function in invasion, where Abl kinase activity is pro-invasive in breast cancer and antiinvasive in HNSCC. Regardless of tumor type, the EGFR/Src pathway is crucial to mediate invasive regardless of divergent cellular response evoked with imatinib-treated HNSCC and breast cancer cell lines.

In Study 2, we further established the crucial role Src in HNSCC metastasis in vitro and in vivo. In addition, we examined effects of pharmacological inhibition of Src/Abl activity with saracatinib on downstream Src effectors and cellular processes important for tumorigenesis. We determined that Src/Abl inhibition reduced HNSCC proliferation and invasion in accordance with impaired invadopodia formation and MMP secretion.

Finally, in Study 3 we determined a functional role for Erk 1/2 activation and phosphorylation of the downstream substrate cortactin in HNSCC patient samples and cell lines, where we showed that Erk 1/2-mediated cortactin phosphorylation modulates HNSCC adhesion and promotes migration by prolonging lamellipodia persistence. 


\section{References}

(1) Rothenberg SM, Ellisen LW. The molecular pathogenesis of head and neck squamous cell carcinoma. J Clin Invest 2012 Jun 1;122(6):1951-1957.

(2) Leemans CR, Braakhuis BJ, Brakenhoff $\mathrm{RH}$. The molecular biology of head and neck cancer. Nat Rev Cancer 2011 Jan;11(1):9-22.

(3) Yavrouian EJ, Sinha UK. Recent advances in biomarkers and potential targeted therapies in head and neck squamous cell carcinoma. ISRN Surg 2012;2012:715743.

(4) Price KA, Cohen EE. Current treatment options for metastatic head and neck cancer. Curr Treat Options Oncol 2012 Mar;13(1):35-46.

(5) Rautava J, Syrjanen S. Biology of human papillomavirus infections in head and neck carcinogenesis. Head Neck Pathol 2012 Jul;6 Suppl 1:S3-15.

(6) Chaudhary AK, Singh M, Sundaram S, Mehrotra R. Role of human papillomavirus and its detection in potentially malignant and malignant head and neck lesions: updated review. Head Neck Oncol 2009 Jun 25;1:22.

(7) O'Rorke MA, Ellison MV, Murray LJ, Moran M, James J, Anderson LA. Human papillomavirus related head and neck cancer survival: A systematic review and meta-analysis. Oral Oncol 2012 Jul 27.

(8) Bonner JA, Harari PM, Giralt J, Azarnia N, Shin DM, Cohen RB, et al. Radiotherapy plus cetuximab for squamous-cell carcinoma of the head and neck. N Engl J Med 2006 Feb 9;354(6):567-578.

(9) Takes RP, Rinaldo A, Silver CE, Haigentz M,Jr, Woolgar JA, Triantafyllou A, et al. Distant metastases from head and neck squamous cell carcinoma. Part I. Basic aspects. Oral Oncol 2012 Sep;48(9):775779.

(10) Nguyen DX, Bos PD, Massague J. Metastasis: from dissemination to organ-specific colonization. Nat Rev Cancer 2009 Apr;9(4):274-284.

(11) Friedl P, Wolf K. Plasticity of cell migration: a multiscale tuning model. J Cell Biol 2010 Jan 11;188(1):11-19.

(12) Sekyrova P, Ostblom J, Andang M. Blebbing as a physical force in cancer EMT - Parallels with mitosis. Semin Cancer Biol 2012 Oct;22(5-6):369-373.

(13) Sabeh F, Shimizu-Hirota R, Weiss SJ. Protease-dependent versus -independent cancer cell invasion programs: three-dimensional amoeboid movement revisited. J Cell Biol 2009 Apr 6;185(1):11-19.

(14) Parri M, Chiarugi P. Rac and Rho GTPases in cancer cell motility control. Cell Commun Signal 2010 Sep 7;8:23.

(15) Crespo P, Calvo F, Sanz-Moreno V. Ras and Rho GTPases on the move: The RasGRF connection. Bioarchitecture 2011 Jul;1(4):200-204.

(16) Friedl P, Wolf K. Proteolytic interstitial cell migration: a five-step process. Cancer Metastasis Rev 2009 Jun;28(1-2):129-135. 
(17) Bravo-Cordero JJ, Hodgson L, Condeelis J. Directed cell invasion and migration during metastasis. Curr Opin Cell Biol 2012 Apr;24(2):277-283.

(18) Friedl P, Alexander S. Cancer invasion and the microenvironment: plasticity and reciprocity. Cell 2011 Nov 23;147(5):992-1009.

(19) Strell C, Rundqvist H, Ostman A. Fibroblasts--a key host cell type in tumor initiation, progression, and metastasis. Ups J Med Sci 2012 May;117(2):187-195.

(20) Dumortier JG, Martin S, Meyer D, Rosa FM, David NB. Collective mesendoderm migration relies on an intrinsic directionality signal transmitted through cell contacts. Proc Natl Acad Sci U S A 2012 Oct 1.

(21) Murphy DA, Courtneidge SA. The 'ins' and 'outs' of podosomes and invadopodia: characteristics, formation and function. Nat Rev Mol Cell Biol 2011 Jun 23;12(7):413-426.

(22) Ridley AJ. Life at the leading edge. Cell 2011 Jun 24;145(7):1012-1022.

(23) Nurnberg A, Kitzing T, Grosse R. Nucleating actin for invasion. Nat Rev Cancer 2011 Mar;11(3):177187.

(24) Sarmiento C, Wang W, Dovas A, Yamaguchi H, Sidani M, El-Sibai M, et al. WASP family members and formin proteins coordinate regulation of cell protrusions in carcinoma cells. J Cell Biol 2008 Mar 24;180(6):1245-1260.

(25) Campellone KG, Welch MD. A nucleator arms race: cellular control of actin assembly. Nat Rev Mol Cell Biol 2010 Apr;11(4):237-251.

(26) Chen WT, Chen JM, Parsons SJ, Parsons JT. Local degradation of fibronectin at sites of expression of the transforming gene product pp60src. Nature $1985 \mathrm{Jul}$ 11-17;316(6024):156-158.

(27) Chen WT. Proteolytic activity of specialized surface protrusions formed at rosette contact sites of transformed cells. J Exp Zool 1989 Aug;251(2):167-185.

(28) Poincloux R, Lizarraga F, Chavrier P. Matrix invasion by tumour cells: a focus on MT1-MMP trafficking to invadopodia. J Cell Sci 2009 Sep 1;122(Pt 17):3015-3024.

(29) Nakahara H, Howard L, Thompson EW, Sato H, Seiki M, Yeh Y, et al. Transmembrane/cytoplasmic domain-mediated membrane type 1-matrix metalloprotease docking to invadopodia is required for cell invasion. Proc Natl Acad Sci U S A 1997 Jul 22;94(15):7959-7964.

(30) Linder S, Wiesner C, Himmel M. Degrading devices: invadosomes in proteolytic cell invasion. Annu Rev Cell Dev Biol 2011 Nov 10;27:185-211.

(31) Bowden ET, Onikoyi E, Slack R, Myoui A, Yoneda T, Yamada KM, et al. Co-localization of cortactin and phosphotyrosine identifies active invadopodia in human breast cancer cells. Exp Cell Res 2006 May 1;312(8):1240-1253.

(32) Yamaguchi H, Lorenz M, Kempiak S, Sarmiento C, Coniglio S, Symons M, et al. Molecular mechanisms of invadopodium formation: the role of the N-WASP-Arp2/3 complex pathway and cofilin. J Cell Biol 2005 Jan 31;168(3):441-452.

(33) Lorenz $\mathrm{M}$, Yamaguchi $\mathrm{H}$, Wang $\mathrm{Y}$, Singer $\mathrm{RH}$, Condeelis J. Imaging sites of $\mathrm{N}$-wasp activity in lamellipodia and invadopodia of carcinoma cells. Curr Biol 2004 Apr 20;14(8):697-703. 
(34) Courtneidge SA, Azucena EF, Pass I, Seals DF, Tesfay L. The SRC substrate Tks5, podosomes (invadopodia), and cancer cell invasion. Cold Spring Harb Symp Quant Biol 2005;70:167-171.

(35) Ayala I, Giacchetti G, Caldieri G, Attanasio F, Mariggio S, Tete S, et al. Faciogenital dysplasia protein Fgd1 regulates invadopodia biogenesis and extracellular matrix degradation and is up-regulated in prostate and breast cancer. Cancer Res 2009 Feb 1;69(3):747-752.

(36) Mader CC, Oser M, Magalhaes MA, Bravo-Cordero JJ, Condeelis J, Koleske AJ, et al. An EGFRSrc-Arg-cortactin pathway mediates functional maturation of invadopodia and breast cancer cell invasion. Cancer Res 2011 Mar 1;71(5):1730-1741.

(37) Oser M, Yamaguchi H, Mader CC, Bravo-Cordero JJ, Arias M, Chen X, et al. Cortactin regulates cofilin and N-WASp activities to control the stages of invadopodium assembly and maturation. J Cell Biol 2009 Aug 24;186(4):571-587.

(38) Parekh A, Ruppender NS, Branch KM, Sewell-Loftin MK, Lin J, Boyer PD, et al. Sensing and modulation of invadopodia across a wide range of rigidities. Biophys J 2011 Feb 2;100(3):573-582.

(39) Crimaldi L, Courtneidge SA, Gimona M. Tks5 recruits AFAP-110, p190RhoGAP, and cortactin for podosome formation. Exp Cell Res 2009 Sep 10;315(15):2581-2592.

(40) Stylli SS, Stacey TT, Verhagen AM, Xu SS, Pass I, Courtneidge SA, et al. Nck adaptor proteins link Tks5 to invadopodia actin regulation and ECM degradation. J Cell Sci 2009 Aug 1;122(Pt 15):2727-2740.

(41) Oser M, Mader CC, Gil-Henn H, Magalhaes M, Bravo-Cordero JJ, Koleske AJ, et al. Specific tyrosine phosphorylation sites on cortactin regulate Nck1-dependent actin polymerization in invadopodia. J Cell Sci 2010 Nov 1;123(Pt 21):3662-3673.

(42) Cai L, Makhov AM, Schafer DA, Bear JE. Coronin 1B antagonizes cortactin and remodels Arp2/3containing actin branches in lamellipodia. Cell 2008 Sep 5;134(5):828-842.

(43) Schoumacher M, Goldman RD, Louvard D, Vignjevic DM. Actin, microtubules, and vimentin intermediate filaments cooperate for elongation of invadopodia. J Cell Biol 2010 May 3;189(3):541-556.

(44) Lizarraga F, Poincloux R, Romao M, Montagnac G, Le Dez G, Bonne I, et al. Diaphanous-related formins are required for invadopodia formation and invasion of breast tumor cells. Cancer Res 2009 Apr 1;69(7):2792-2800.

(45) Philippar U, Roussos ET, Oser M, Yamaguchi H, Kim HD, Giampieri S, et al. A Mena invasion isoform potentiates EGF-induced carcinoma cell invasion and metastasis. Dev Cell 2008 Dec;15(6):813828.

(46) Gertler F, Condeelis J. Metastasis: tumor cells becoming MENAcing. Trends Cell Biol 2011 Feb;21(2):81-90.

(47) Mukhopadhyay UK, Eves R, Jia L, Mooney P, Mak AS. p53 suppresses Src-induced podosome and rosette formation and cellular invasiveness through the upregulation of caldesmon. Mol Cell Biol 2009 Jun;29(11):3088-3098.

(48) Li A, Dawson JC, Forero-Vargas M, Spence HJ, Yu X, Konig I, et al. The actin-bundling protein fascin stabilizes actin in invadopodia and potentiates protrusive invasion. Curr Biol $2010 \mathrm{Feb}$ 23;20(4):339-345. 
(49) Mueller SC, Yeh Y, Chen WT. Tyrosine phosphorylation of membrane proteins mediates cellular invasion by transformed cells. J Cell Biol 1992 Dec;119(5):1309-1325.

(50) Bowden ET, Barth M, Thomas D, Glazer RI, Mueller SC. An invasion-related complex of cortactin, paxillin and PKCmu associates with invadopodia at sites of extracellular matrix degradation. Oncogene 1999 Aug 5;18(31):4440-4449.

(51) Alexander NR, Branch KM, Parekh A, Clark ES, Iwueke IC, Guelcher SA, et al. Extracellular matrix rigidity promotes invadopodia activity. Curr Biol 2008 Sep 9;18(17):1295-1299.

(52) Wolf K, Friedl P. Mapping proteolytic cancer cell-extracellular matrix interfaces. Clin Exp Metastasis 2009;26(4):289-298.

(53) Tolde O, Rosel D, Vesely P, Folk P, Brabek J. The structure of invadopodia in a complex 3D environment. Eur J Cell Biol 2010 Sep;89(9):674-680.

(54) Monsky WL, Lin CY, Aoyama A, Kelly T, Akiyama SK, Mueller SC, et al. A potential marker protease of invasiveness, seprase, is localized on invadopodia of human malignant melanoma cells. Cancer Res 1994 Nov 1;54(21):5702-5710.

(55) Monsky WL, Chen WT. Proteases of cell adhesion proteins in cancer. Semin Cancer Biol 1993 Aug;4(4):251-258.

(56) Redondo-Munoz J, Escobar-Diaz E, Samaniego R, Terol MJ, Garcia-Marco JA, Garcia-Pardo A. MMP-9 in B-cell chronic lymphocytic leukemia is up-regulated by alpha4beta1 integrin or CXCR4 engagement via distinct signaling pathways, localizes to podosomes, and is involved in cell invasion and migration. Blood 2006 Nov 1;108(9):3143-3151.

(57) Artym VV, Zhang Y, Seillier-Moiseiwitsch F, Yamada KM, Mueller SC. Dynamic interactions of cortactin and membrane type 1 matrix metalloproteinase at invadopodia: defining the stages of invadopodia formation and function. Cancer Res 2006 Mar 15;66(6):3034-3043.

(58) Ghersi G, Dong H, Goldstein LA, Yeh Y, Hakkinen L, Larjava HS, et al. Regulation of fibroblast migration on collagenous matrix by a cell surface peptidase complex. J Biol Chem 2002 Aug 9;277(32):29231-29241.

(59) Kindzelskii AL, Amhad I, Keller D, Zhou MJ, Haugland RP, Garni-Wagner BA, et al. Pericellular proteolysis by leukocytes and tumor cells on substrates: focal activation and the role of urokinase-type plasminogen activator. Histochem Cell Biol 2004 Apr;121(4):299-310.

(60) Sabeh F, Ota I, Holmbeck K, Birkedal-Hansen H, Soloway P, Balbin M, et al. Tumor cell traffic through the extracellular matrix is controlled by the membrane-anchored collagenase MT1-MMP. J Cell Biol 2004 Nov 22;167(4):769-781.

(61) Hotary K, Li XY, Allen E, Stevens SL, Weiss SJ. A cancer cell metalloprotease triad regulates the basement membrane transmigration program. Genes Dev 2006 Oct 1;20(19):2673-2686.

(62) Steffen A, Le Dez G, Poincloux R, Recchi C, Nassoy P, Rottner K, et al. MT1-MMP-dependent invasion is regulated by TI-VAMP/VAMP7. Curr Biol 2008 Jun 24;18(12):926-931. 
(63) Albrechtsen R, Stautz D, Sanjay A, Kveiborg M, Wewer UM. Extracellular engagement of ADAM12 induces clusters of invadopodia with localized ectodomain shedding activity. Exp Cell Res 2011 Jan 15;317(2):195-209.

(64) Caldieri G, Giacchetti G, Beznoussenko G, Attanasio F, Ayala I, Buccione R. Invadopodia biogenesis is regulated by caveolin-mediated modulation of membrane cholesterol levels. J Cell Mol Med 2009 Aug;13(8B):1728-1740.

(65) Caldieri G, Buccione R. Aiming for invadopodia: organizing polarized delivery at sites of invasion. Trends Cell Biol 2010 Feb;20(2):64-70.

(66) Yamaguchi H, Takeo Y, Yoshida S, Kouchi Z, Nakamura Y, Fukami K. Lipid rafts and caveolin-1 are required for invadopodia formation and extracellular matrix degradation by human breast cancer cells. Cancer Res 2009 Nov 15;69(22):8594-8602.

(67) Bravo-Cordero JJ, Marrero-Diaz R, Megias D, Genis L, Garcia-Grande A, Garcia MA, et al. MT1MMP proinvasive activity is regulated by a novel Rab8-dependent exocytic pathway. EMBO J 2007 Mar $21 ; 26(6): 1499-1510$.

(68) Sakurai-Yageta M, Recchi C, Le Dez G, Sibarita JB, Daviet L, Camonis J, et al. The interaction of IQGAP1 with the exocyst complex is required for tumor cell invasion downstream of Cdc42 and RhoA. J Cell Biol 2008 Jun 16;181(6):985-998.

(69) Yu X, Zech T, McDonald L, Gonzalez EG, Li A, Macpherson I, et al. N-WASP coordinates the delivery and F-actin-mediated capture of MT1-MMP at invasive pseudopods. J Cell Biol 2012 Oct 29;199(3):527-544.

(70) Clark ES, Whigham AS, Yarbrough WG, Weaver AM. Cortactin is an essential regulator of matrix metalloproteinase secretion and extracellular matrix degradation in invadopodia. Cancer Res 2007 May 1;67(9):4227-4235.

(71) Sutoh M, Hashimoto Y, Yoneyama T, Yamamoto H, Hatakeyama S, Koie T, et al. Invadopodia formation by bladder tumor cells. Oncol Res 2010;19(2):85-92.

(72) Yamamoto H, Sutoh M, Hatakeyama S, Hashimoto Y, Yoneyama T, Koie T, et al. Requirement for FBP17 in invadopodia formation by invasive bladder tumor cells. J Urol 2011 May;185(5):1930-1938.

(73) Stylli SS, Kaye AH, Lock P. Invadopodia: at the cutting edge of tumour invasion. J Clin Neurosci 2008 Jul;15(7):725-737.

(74) Gligorijevic B, Wyckoff J, Yamaguchi H, Wang Y, Roussos ET, Condeelis J. N-WASP-mediated invadopodium formation is involved in intravasation and lung metastasis of mammary tumors. $J$ Cell Sci 2012 Feb 1;125(Pt 3):724-734.

(75) Wyckoff J, Gligorijevic B, Entenberg D, Segall J, Condeelis J. High-resolution multiphoton imaging of tumors in vivo. Cold Spring Harb Protoc 2011 Oct 1;2011(10):1167-1184.

(76) Quintavalle M, Elia L, Condorelli G, Courtneidge SA. MicroRNA control of podosome formation in vascular smooth muscle cells in vivo and in vitro. J Cell Biol 2010 Apr 5;189(1):13-22. 
(77) Magalhaes MA, Larson DR, Mader CC, Bravo-Cordero JJ, Gil-Henn H, Oser M, et al. Cortactin phosphorylation regulates cell invasion through a pH-dependent pathway. J Cell Biol 2011 Nov 28;195(5):903-920.

(78) Destaing O, Block MR, Planus E, Albiges-Rizo C. Invadosome regulation by adhesion signaling. Curr Opin Cell Biol 2011 Oct;23(5):597-606.

(79) Wu H, Reynolds AB, Kanner SB, Vines RR, Parsons JT. Identification and characterization of a novel cytoskeleton-associated pp60src substrate. Mol Cell Biol 1991 Oct;11(10):5113-5124.

(80) Kirkbride KC, Sung BH, Sinha S, Weaver AM. Cortactin: a multifunctional regulator of cellular invasiveness. Cell Adh Migr 2011 Mar-Apr;5(2):187-198.

(81) Akervall JA, Jin Y, Wennerberg JP, Zatterstrom UK, Kjellen E, Mertens F, et al. Chromosomal abnormalities involving $11 \mathrm{q} 13$ are associated with poor prognosis in patients with squamous cell carcinoma of the head and neck. Cancer 1995 Sep 1;76(5):853-859.

(82) Rodrigo JP, Garcia-Carracedo D, Garcia LA, Menendez S, Allonca E, Gonzalez MV, et al. Distinctive clinicopathological associations of amplification of the cortactin gene at $11 \mathrm{q} 13$ in head and neck squamous cell carcinomas. J Pathol 2009 Mar;217(4):516-523.

(83) Ormandy CJ, Musgrove EA, Hui R, Daly RJ, Sutherland RL. Cyclin D1, EMS1 and 11q13 amplification in breast cancer. Breast Cancer Res Treat 2003 Apr;78(3):323-335.

(84) Buday L, Downward J. Roles of cortactin in tumor pathogenesis. Biochim Biophys Acta 2007 Jun;1775(2):263-273.

(85) Zaharieva BM, Simon R, Diener PA, Ackermann D, Maurer R, Alund G, et al. High-throughput tissue microarray analysis of 11 q13 gene amplification (CCND1, FGF3, FGF4, EMS1) in urinary bladder cancer. J Pathol 2003 Dec;201(4):603-608.

(86) Faoro L, Singleton PA, Cervantes GM, Lennon FE, Choong NW, Kanteti R, et al. EphA2 mutation in lung squamous cell carcinoma promotes increased cell survival, cell invasion, focal adhesions, and mammalian target of rapamycin activation. J Biol Chem 2010 Jun 11;285(24):18575-18585.

(87) Meredith SD, Levine PA, Burns JA, Gaffey MJ, Boyd JC, Weiss LM, et al. Chromosome 11q13 amplification in head and neck squamous cell carcinoma. Association with poor prognosis. Arch Otolaryngol Head Neck Surg 1995 Jul;121(7):790-794.

(88) Sugahara K, Michikawa Y, Ishikawa K, Shoji Y, Iwakawa M, Shibahara T, et al. Combination effects of distinct cores in $11 \mathrm{q} 13$ amplification region on cervical lymph node metastasis of oral squamous cell carcinoma. Int J Oncol 2011 Oct;39(4):761-769.

(89) Hui R, Ball JR, Macmillan RD, Kenny FS, Prall OW, Campbell DH, et al. EMS1 gene expression in primary breast cancer: relationship to cyclin D1 and oestrogen receptor expression and patient survival. Oncogene 1998 Aug 27;17(8):1053-1059.

(90) Li Y, Tondravi M, Liu J, Smith E, Haudenschild CC, Kaczmarek M, et al. Cortactin potentiates bone metastasis of breast cancer cells. Cancer Res 2001 Sep 15;61(18):6906-6911.

(91) Cowieson NP, King G, Cookson D, Ross I, Huber T, Hume DA, et al. Cortactin adopts a globular conformation and bundles actin into sheets. J Biol Chem 2008 Jun 6;283(23):16187-16193. 
(92) Weed SA, Parsons JT. Cortactin: coupling membrane dynamics to cortical actin assembly. Oncogene 2001 Oct 1;20(44):6418-6434.

(93) Goode BL, Rodal AA, Barnes G, Drubin DG. Activation of the Arp2/3 complex by the actin filament binding protein Abp1p. J Cell Biol 2001 Apr 30;153(3):627-634.

(94) Skoble J, Portnoy DA, Welch MD. Three regions within ActA promote Arp2/3 complex-mediated actin nucleation and Listeria monocytogenes motility. J Cell Biol 2000 Aug 7;150(3):527-538.

(95) Lechler T, Shevchenko A, Li R. Direct involvement of yeast type I myosins in Cdc42-dependent actin polymerization. J Cell Biol 2000 Jan 24;148(2):363-373.

(96) Machesky LM, Mullins RD, Higgs HN, Kaiser DA, Blanchoin L, May RC, et al. Scar, a WASp-related protein, activates nucleation of actin filaments by the Arp2/3 complex. Proc Natl Acad Sci U S A 1999 Mar 30;96(7):3739-3744.

(97) Weaver AM, Karginov AV, Kinley AW, Weed SA, Li Y, Parsons JT, et al. Cortactin promotes and stabilizes Arp2/3-induced actin filament network formation. Curr Biol 2001 Mar 6;11(5):370-374.

(98) Kinley AW, Weed SA, Weaver AM, Karginov AV, Bissonette E, Cooper JA, et al. Cortactin interacts with WIP in regulating Arp2/3 activation and membrane protrusion. Curr Biol 2003 Mar 4;13(5):384-393.

(99) Weaver AM, Heuser JE, Karginov AV, Lee WL, Parsons JT, Cooper JA. Interaction of cortactin and N-WASp with Arp2/3 complex. Curr Biol 2002 Aug 6;12(15):1270-1278.

(100) Tehrani S, Tomasevic N, Weed S, Sakowicz R, Cooper JA. Src phosphorylation of cortactin enhances actin assembly. Proc Natl Acad Sci U S A 2007 Jul 17;104(29):11933-11938.

(101) Sparks AB, Hoffman NG, McConnell SJ, Fowlkes DM, Kay BK. Cloning of ligand targets: systematic isolation of SH3 domain-containing proteins. Nat Biotechnol 1996 Jun;14(6):741-744.

(102) Zhang Y, Zhang M, Dong H, Yong S, Li X, Olashaw N, et al. Deacetylation of cortactin by SIRT1 promotes cell migration. Oncogene 2009 Jan 22;28(3):445-460.

(103) Luxton GW, Gundersen GG. HDAC6-pack: cortactin acetylation joins the brew. Dev Cell 2007 Aug;13(2):161-162.

(104) Zhang X, Yuan Z, Zhang Y, Yong S, Salas-Burgos A, Koomen J, et al. HDAC6 modulates cell motility by altering the acetylation level of cortactin. Mol Cell 2007 Jul 20;27(2):197-213.

(105) Kaluza D, Kroll J, Gesierich S, Yao TP, Boon RA, Hergenreider E, et al. Class Ilb HDAC6 regulates endothelial cell migration and angiogenesis by deacetylation of cortactin. EMBO J 2011 Aug 16;30(20):4142-4156.

(106) Nakane K, Fujita Y, Terazawa R, Atsumi Y, Kato T, Nozawa Y, et al. Inhibition of cortactin and SIRT1 expression attenuates migration and invasion of prostate cancer DU145 cells. Int J Urol 2012 Jan;19(1):71-79.

(107) Rey M, Irondelle M, Waharte F, Lizarraga F, Chavrier P. HDAC6 is required for invadopodia activity and invasion by breast tumor cells. Eur J Cell Biol 2011 Feb-Mar;90(2-3):128-135.

(108) Perrin BJ, Amann KJ, Huttenlocher A. Proteolysis of cortactin by calpain regulates membrane protrusion during cell migration. Mol Biol Cell 2006 Jan;17(1):239-250. 
(109) Huang C, Liu J, Haudenschild CC, Zhan X. The role of tyrosine phosphorylation of cortactin in the locomotion of endothelial cells. J Biol Chem 1998 Oct 2;273(40):25770-25776.

(110) Boyle SN, Michaud GA, Schweitzer B, Predki PF, Koleske AJ. A critical role for cortactin phosphorylation by Abl-family kinases in PDGF-induced dorsal-wave formation. Curr Biol 2007 Mar 6;17(5):445-451.

(111) Lapetina S, Mader CC, Machida K, Mayer BJ, Koleske AJ. Arg interacts with cortactin to promote adhesion-dependent cell edge protrusion. J Cell Biol 2009 May 4;185(3):503-519.

(112) Zhang X, Shrikhande U, Alicie BM, Zhou Q, Geahlen RL. Role of the protein tyrosine kinase Syk in regulating cell-cell adhesion and motility in breast cancer cells. Mol Cancer Res 2009 May;7(5):634-644.

(113) Huang J, Asawa T, Takato T, Sakai R. Cooperative roles of Fyn and cortactin in cell migration of metastatic murine melanoma. J Biol Chem 2003 Nov 28;278(48):48367-48376.

(114) Bougneres L, Girardin SE, Weed SA, Karginov AV, Olivo-Marin JC, Parsons JT, et al. Cortactin and Crk cooperate to trigger actin polymerization during Shigella invasion of epithelial cells. J Cell Biol 2004 Jul 19;166(2):225-235.

(115) Hayes KE, Walk EL, Ammer AG, Kelley LC, Martin KH, Weed SA. Ableson kinases negatively regulate invadopodia function and invasion in head and neck squamous cell carcinoma by inhibiting an HB-EGF autocrine loop. Oncogene 2012 Nov 12.

(116) Kelley LC, Ammer AG, Hayes KE, Martin KH, Machida K, Jia L, et al. Oncogenic Src requires a wild-type counterpart to regulate invadopodia maturation. J Cell Sci 2010 Nov 15;123(Pt 22):3923-3932.

(117) Head JA, Jiang D, Li M, Zorn LJ, Schaefer EM, Parsons JT, et al. Cortactin tyrosine phosphorylation requires Rac1 activity and association with the cortical actin cytoskeleton. Mol Biol Cell 2003 Aug;14(8):3216-3229.

(118) Kruchten AE, Krueger EW, Wang Y, McNiven MA. Distinct phospho-forms of cortactin differentially regulate actin polymerization and focal adhesions. Am J Physiol Cell Physiol 2008 Nov;295(5):C1113-22.

(119) Kowalski JR, Egile C, Gil S, Snapper SB, Li R, Thomas SM. Cortactin regulates cell migration through activation of N-WASP. J Cell Sci 2005 Jan 1;118(Pt 1):79-87.

(120) Stuible M, Dube N, Tremblay ML. PTP1B regulates cortactin tyrosine phosphorylation by targeting Tyr446. J Biol Chem 2008 Jun 6;283(23):15740-15746.

(121) Martinez-Quiles N, Ho HY, Kirschner MW, Ramesh N, Geha RS. Erk/Src phosphorylation of cortactin acts as a switch on-switch off mechanism that controls its ability to activate N-WASP. Mol Cell Biol 2004 Jun;24(12):5269-5280.

(122) Campbell DH, Sutherland RL, Daly RJ. Signaling pathways and structural domains required for phosphorylation of EMS1/cortactin. Cancer Res 1999 Oct 15;59(20):5376-5385.

(123) Kelley LC, Hayes KE, Ammer AG, Martin KH, Weed SA. Cortactin phosphorylated by ERK1/2 localizes to sites of dynamic actin regulation and is required for carcinoma lamellipodia persistence. PLoS One 2010 Nov 4;5(11):e13847. 
(124) Kelley LC, Hayes KE, Ammer AG, Martin KH, Weed SA. Revisiting the ERK/Src cortactin switch. Commun Integr Biol 2011 Mar;4(2):205-207.

(125) Grassart A, Meas-Yedid V, Dufour A, Olivo-Marin JC, Dautry-Varsat A, Sauvonnet N. Pak1 phosphorylation enhances cortactin-N-WASP interaction in clathrin-caveolin-independent endocytosis. Traffic 2010 Aug;11(8):1079-1091.

(126) Webb BA, Zhou S, Eves R, Shen L, Jia L, Mak AS. Phosphorylation of cortactin by p21-activated kinase. Arch Biochem Biophys 2006 Dec 15;456(2):183-193.

(127) Eiseler T, Hausser A, De Kimpe L, Van Lint J, Pfizenmaier K. Protein kinase D controls actin polymerization and cell motility through phosphorylation of cortactin. J Biol Chem 2010 Jun $11 ; 285(24): 18672-18683$.

(128) Eiseler T, Schmid MA, Topbas F, Pfizenmaier K, Hausser A. PKD is recruited to sites of actin remodelling at the leading edge and negatively regulates cell migration. FEBS Lett 2007 Sep 4;581(22):4279-4287.

(129) De Kimpe L, Janssens K, Derua R, Armacki M, Goicoechea S, Otey C, et al. Characterization of cortactin as an in vivo protein kinase $D$ substrate: interdependence of sites and potentiation by Src. Cell Signal 2009 Feb;21(2):253-263.

(130) Martin KH, Jeffery ED, Grigera PR, Shabanowitz J, Hunt DF, Parsons JT. Cortactin phosphorylation sites mapped by mass spectrometry. J Cell Sci 2006 Jul 15;119(Pt 14):2851-2853.

(131) Agerer F, Lux S, Michel A, Rohde M, Ohlsen K, Hauck CR. Cellular invasion by Staphylococcus aureus reveals a functional link between focal adhesion kinase and cortactin in integrin-mediated internalisation. J Cell Sci 2005 May 15;118(Pt 10):2189-2200.

(132) Sachdev S, Bu Y, Gelman IH. Paxillin-Y118 phosphorylation contributes to the control of Srcinduced anchorage-independent growth by FAK and adhesion. BMC Cancer 2009 Jan 12;9:12.

(133) Tomar A, Lawson C, Ghassemian M, Schlaepfer DD. Cortactin as a target for FAK in the regulation of focal adhesion dynamics. PLoS One 2012;7(8):e44041.

(134) Wang W, Liu Y, Liao K. Tyrosine phosphorylation of cortactin by the FAK-Src complex at focal adhesions regulates cell motility. BMC Cell Biol 2011 Nov 13;12:49.

(135) Eke I, Deuse Y, Hehlgans S, Gurtner K, Krause M, Baumann M, et al. beta(1)Integrin/FAK/cortactin signaling is essential for human head and neck cancer resistance to radiotherapy. J Clin Invest $2012 \mathrm{Apr}$ 2;122(4):1529-1540.

(136) Ren G, Crampton MS, Yap AS. Cortactin: Coordinating adhesion and the actin cytoskeleton at cellular protrusions. Cell Motil Cytoskeleton 2009 Oct;66(10):865-873.

(137) Ammer AG, Weed SA. Cortactin branches out: roles in regulating protrusive actin dynamics. Cell Motil Cytoskeleton 2008 Sep;65(9):687-707.

(138) MacGrath SM, Koleske AJ. Cortactin in cell migration and cancer at a glance. J Cell Sci 2012 Apr 1;125(Pt 7):1621-1626. 
(139) Ayala I, Baldassarre M, Giacchetti G, Caldieri G, Tete S, Luini A, et al. Multiple regulatory inputs converge on cortactin to control invadopodia biogenesis and extracellular matrix degradation. J Cell Sci 2008 Feb 1;121(Pt 3):369-378.

(140) Foley J, Nickerson N, Riese DJ,2nd, Hollenhorst PC, Lorch G, Foley AM. At the crossroads: EGFR and PTHrP signaling in cancer-mediated diseases of bone. Odontology 2012 Jul;100(2):109-129.

(141) Tejani MA, Cohen RB, Mehra R. The contribution of cetuximab in the treatment of recurrent and/or metastatic head and neck cancer. Biologics 2010 Aug 9;4:173-185.

(142) Eccles SA. The epidermal growth factor receptor/Erb-B/HER family in normal and malignant breast biology. Int J Dev Biol 2011;55(7-9):685-696.

(143) Wilson KJ, Gilmore JL, Foley J, Lemmon MA, Riese DJ,2nd. Functional selectivity of EGF family peptide growth factors: implications for cancer. Pharmacol Ther 2009 Apr;122(1):1-8.

(144) Stokes A, Joutsa J, Ala-Aho R, Pitchers M, Pennington CJ, Martin C, et al. Expression profiles and clinical correlations of degradome components in the tumor microenvironment of head and neck squamous cell carcinoma. Clin Cancer Res 2010 Apr 1;16(7):2022-2035.

(145) Sahin U, Weskamp G, Kelly K, Zhou HM, Higashiyama S, Peschon J, et al. Distinct roles for ADAM10 and ADAM17 in ectodomain shedding of six EGFR ligands. J Cell Biol 2004 Mar 1;164(5):769779.

(146) Iwamoto R, Mekada E. Heparin-binding EGF-like growth factor: a juxtacrine growth factor. Cytokine Growth Factor Rev 2000 Dec;11(4):335-344.

(147) Busser B, Sancey L, Brambilla E, Coll JL, Hurbin A. The multiple roles of amphiregulin in human cancer. Biochim Biophys Acta 2011 Dec;1816(2):119-131.

(148) Dasgupta S, Tripathi PK, Qin H, Bhattacharya-Chatterjee M, Valentino J, Chatterjee SK. Identification of molecular targets for immunotherapy of patients with head and neck squamous cell carcinoma. Oral Oncol 2006 Mar;42(3):306-316.

(149) Dawson JP, Berger MB, Lin CC, Schlessinger J, Lemmon MA, Ferguson KM. Epidermal growth factor receptor dimerization and activation require ligand-induced conformational changes in the dimer interface. Mol Cell Biol 2005 Sep;25(17):7734-7742.

(150) Endres NF, Engel K, Das R, Kovacs E, Kuriyan J. Regulation of the catalytic activity of the EGF receptor. Curr Opin Struct Biol 2011 Dec;21(6):777-784.

(151) Yarden Y, Sliwkowski MX. Untangling the ErbB signalling network. Nat Rev Mol Cell Biol 2001 Feb;2(2):127-137.

(152) Roberts PJ, Der CJ. Targeting the Raf-MEK-ERK mitogen-activated protein kinase cascade for the treatment of cancer. Oncogene 2007 May 14;26(22):3291-3310.

(153) Henson ES, Gibson SB. Surviving cell death through epidermal growth factor (EGF) signal transduction pathways: implications for cancer therapy. Cell Signal 2006 Dec;18(12):2089-2097.

(154) Kisseleva T, Bhattacharya S, Braunstein J, Schindler CW. Signaling through the JAK/STAT pathway, recent advances and future challenges. Gene 2002 Feb 20;285(1-2):1-24. 
(155) Freudlsperger C, Burnett JR, Friedman JA, Kannabiran VR, Chen Z, Van Waes C. EGFR-PI3KAKT-mTOR signaling in head and neck squamous cell carcinomas: attractive targets for molecularoriented therapy. Expert Opin Ther Targets 2011 Jan;15(1):63-74.

(156) Visser Smit GD, Place TL, Cole SL, Clausen KA, Vemuganti S, Zhang G, et al. Cbl controls EGFR fate by regulating early endosome fusion. Sci Signal 2009 Dec 22;2(102):ra86.

(157) Sorkin A, Goh LK. Endocytosis and intracellular trafficking of ErbBs. Exp Cell Res 2009 Feb 15;315(4):683-696.

(158) Eden ER, Huang F, Sorkin A, Futter CE. The role of EGF receptor ubiquitination in regulating its intracellular traffic. Traffic 2012 Feb;13(2):329-337.

(159) Waterman H, Yarden Y. Molecular mechanisms underlying endocytosis and sorting of ErbB receptor tyrosine kinases. FEBS Lett 2001 Feb 16;490(3):142-152.

(160) Wang YN, Wang H, Yamaguchi H, Lee HJ, Lee HH, Hung MC. COPI-mediated retrograde trafficking from the Golgi to the ER regulates EGFR nuclear transport. Biochem Biophys Res Commun 2010 Sep 3;399(4):498-504.

(161) Giri DK, Ali-Seyed M, Li LY, Lee DF, Ling P, Bartholomeusz G, et al. Endosomal transport of ErbB2: mechanism for nuclear entry of the cell surface receptor. Mol Cell Biol 2005 Dec;25(24):11005-11018.

(162) Brand TM, lida M, Li C, Wheeler DL. The nuclear epidermal growth factor receptor signaling network and its role in cancer. Discov Med 2011 Nov;12(66):419-432.

(163) Han W, Lo HW. Landscape of EGFR signaling network in human cancers: biology and therapeutic response in relation to receptor subcellular locations. Cancer Lett 2012 May 28;318(2):124-134.

(164) Plattner R, Koleske AJ, Kazlauskas A, Pendergast AM. Bidirectional signaling links the Abelson kinases to the platelet-derived growth factor receptor. Mol Cell Biol 2004 Mar;24(6):2573-2583.

(165) Plattner R, Kadlec L, DeMali KA, Kazlauskas A, Pendergast AM. c-Abl is activated by growth factors and Src family kinases and has a role in the cellular response to PDGF. Genes Dev 1999 Sep 15;13(18):2400-2411.

(166) Murillo MM, del Castillo G, Sanchez A, Fernandez M, Fabregat I. Involvement of EGF receptor and C-Src in the survival signals induced by TGF-beta1 in hepatocytes. Oncogene 2005 Jun 30;24(28):45804587.

(167) Xue C, Wyckoff J, Liang F, Sidani M, Violini S, Tsai KL, et al. Epidermal growth factor receptor overexpression results in increased tumor cell motility in vivo coordinately with enhanced intravasation and metastasis. Cancer Res 2006 Jan 1;66(1):192-197.

(168) Dise RS, Frey MR, Whitehead RH, Polk DB. Epidermal growth factor stimulates Rac activation through Src and phosphatidylinositol 3-kinase to promote colonic epithelial cell migration. Am J Physiol Gastrointest Liver Physiol 2008 Jan;294(1):G276-85.

(169) Hwang YS, Park KK, Chung WY. Invadopodia formation in oral squamous cell carcinoma: the role of epidermal growth factor receptor signalling. Arch Oral Biol 2012 Apr;57(4):335-343. 
(170) Kalyankrishna S, Grandis JR. Epidermal growth factor receptor biology in head and neck cancer. J Clin Oncol 2006 Jun 10;24(17):2666-2672.

(171) O-charoenrat P, Modjtahedi H, Rhys-Evans P, Court WJ, Box GM, Eccles SA. Epidermal growth factor-like ligands differentially up-regulate matrix metalloproteinase 9 in head and neck squamous carcinoma cells. Cancer Res 2000 Feb 15;60(4):1121-1128.

(172) Zuo JH, Zhu W, Li MY, Li XH, Yi H, Zeng GQ, et al. Activation of EGFR promotes squamous carcinoma SCC10A cell migration and invasion via inducing EMT-like phenotype change and MMP-9mediated degradation of E-cadherin. J Cell Biochem 2011 Sep;112(9):2508-2517.

(173) Taoudi Benchekroun M, Saintigny P, Thomas SM, El-Naggar AK, Papadimitrakopoulou V, Ren H, et al. Epidermal growth factor receptor expression and gene copy number in the risk of oral cancer. Cancer Prev Res (Phila) 2010 Jul;3(7):800-809.

(174) Rubin Grandis J, Melhem MF, Gooding WE, Day R, Holst VA, Wagener MM, et al. Levels of TGFalpha and EGFR protein in head and neck squamous cell carcinoma and patient survival. J Natl Cancer Inst 1998 Jun 3;90(11):824-832.

(175) Shirasuna K, Hayashido Y, Sugiyama M, Yoshioka H, Matsuya T. Immunohistochemical localization of epidermal growth factor (EGF) and EGF receptor in human oral mucosa and its malignancy. Virchows Arch A Pathol Anat Histopathol 1991;418(4):349-353.

(176) Wheeler S, Siwak DR, Chai R, LaValle C, Seethala RR, Wang L, et al. Tumor epidermal growth factor receptor and EGFR PY1068 are independent prognostic indicators for head and neck squamous cell carcinoma. Clin Cancer Res 2012 Apr 15;18(8):2278-2289.

(177) Sok JC, Coppelli FM, Thomas SM, Lango MN, Xi S, Hunt JL, et al. Mutant epidermal growth factor receptor (EGFRvIII) contributes to head and neck cancer growth and resistance to EGFR targeting. Clin Cancer Res 2006 Sep 1;12(17):5064-5073.

(178) Lee JW, Soung YH, Kim SY, Nam HK, Park WS, Nam SW, et al. Somatic mutations of EGFR gene in squamous cell carcinoma of the head and neck. Clin Cancer Res 2005 Apr 15;11(8):2879-2882.

(179) Szabo B, Nelhubel GA, Karpati A, Kenessey I, Jori B, Szekely C, et al. Clinical significance of genetic alterations and expression of epidermal growth factor receptor (EGFR) in head and neck squamous cell carcinomas. Oral Oncol 2011 Jun;47(6):487-496.

(180) Omidfar K, Shirvani Z. Single domain antibodies: a new concept for epidermal growth factor receptor and EGFRvIII targeting. DNA Cell Biol 2012 Jun;31(6):1015-1026.

(181) Nedergaard MK, Hedegaard CJ, Poulsen HS. Targeting the epidermal growth factor receptor in solid tumor malignancies. BioDrugs 2012 Apr 1;26(2):83-99.

(182) Foley J, Nickerson NK, Nam S, Allen KT, Gilmore JL, Nephew KP, et al. EGFR signaling in breast cancer: bad to the bone. Semin Cell Dev Biol 2010 Dec;21(9):951-960.

(183) Sharma SV, Bell DW, Settleman J, Haber DA. Epidermal growth factor receptor mutations in lung cancer. Nat Rev Cancer 2007 Mar;7(3):169-181. 
(184) Wong AJ, Bigner SH, Bigner DD, Kinzler KW, Hamilton SR, Vogelstein B. Increased expression of the epidermal growth factor receptor gene in malignant gliomas is invariably associated with gene amplification. Proc Natl Acad Sci U S A 1987 Oct;84(19):6899-6903.

(185) Kundu SK, Nestor M. Targeted therapy in head and neck cancer. Tumour Biol 2012 Jun;33(3):707721.

(186) Reeves TD, Hill EG, Armeson KE, Gillespie MB. Cetuximab therapy for head and neck squamous cell carcinoma: a systematic review of the data. Otolaryngol Head Neck Surg 2011 May;144(5):676-684.

(187) Vermorken JB, Mesia R, Rivera F, Remenar E, Kawecki A, Rottey S, et al. Platinum-based chemotherapy plus cetuximab in head and neck cancer. N Engl J Med 2008 Sep 11;359(11):1116-1127.

(188) Cripps C, Winquist E, Devries MC, Stys-Norman D, Gilbert R, Head and Neck Cancer Disease Site Group. Epidermal growth factor receptor targeted therapy in stages III and IV head and neck cancer. Curr Oncol 2010 Jun;17(3):37-48.

(189) Seshacharyulu P, Ponnusamy MP, Haridas D, Jain M, Ganti AK, Batra SK. Targeting the EGFR signaling pathway in cancer therapy. Expert Opin Ther Targets 2012 Jan;16(1):15-31.

(190) Johnson GL, Lapadat R. Mitogen-activated protein kinase pathways mediated by ERK, JNK, and p38 protein kinases. Science 2002 Dec 6;298(5600):1911-1912.

(191) Krishna M, Narang $\mathrm{H}$. The complexity of mitogen-activated protein kinases (MAPKs) made simple. Cell Mol Life Sci 2008 Nov;65(22):3525-3544.

(192) Garai A, Zeke A, Gogl G, Toro I, Fordos F, Blankenburg H, et al. Specificity of linear motifs that bind to a common mitogen-activated protein kinase docking groove. Sci Signal 2012 Oct 9;5(245):ra74.

(193) Zhang F, Strand A, Robbins D, Cobb MH, Goldsmith EJ. Atomic structure of the MAP kinase ERK2 at 2.3 A resolution. Nature 1994 Feb 24;367(6465):704-711.

(194) Wilson KP, Fitzgibbon MJ, Caron PR, Griffith JP, Chen W, McCaffrey PG, et al. Crystal structure of p38 mitogen-activated protein kinase. J Biol Chem 1996 Nov 1;271(44):27696-27700.

(195) Wortzel I, Seger R. The ERK Cascade: Distinct Functions within Various Subcellular Organelles. Genes Cancer 2011 Mar;2(3):195-209.

(196) Chetram MA, Hinton CV. PTEN regulation of ERK1/2 signaling in cancer. J Recept Signal Transduct Res 2012 Aug;32(4):190-195.

(197) Wood KW, Sarnecki C, Roberts TM, Blenis J. ras mediates nerve growth factor receptor modulation of three signal-transducing protein kinases: MAP kinase, Raf-1, and RSK. Cell 1992 Mar 20;68(6):10411050.

(198) Kyriakis JM, App H, Zhang XF, Banerjee P, Brautigan DL, Rapp UR, et al. Raf-1 activates MAP kinase-kinase. Nature 1992 Jul 30;358(6385):417-421.

(199) Kolch W. Meaningful relationships: the regulation of the Ras/Raf/MEK/ERK pathway by protein interactions. Biochem J 2000 Oct 15;351 Pt 2:289-305.

(200) Morrison DK, Davis RJ. Regulation of MAP kinase signaling modules by scaffold proteins in mammals. Annu Rev Cell Dev Biol 2003;19:91-118. 
(201) Yu W, Fantl WJ, Harrowe G, Williams LT. Regulation of the MAP kinase pathway by mammalian Ksr through direct interaction with MEK and ERK. Curr Biol 1998 Jan 1;8(1):56-64.

(202) Haystead TA, Dent P, Wu J, Haystead CM, Sturgill TW. Ordered phosphorylation of p42mapk by MAP kinase kinase. FEBS Lett 1992 Jul 13;306(1):17-22.

(203) Payne DM, Rossomando AJ, Martino P, Erickson AK, Her JH, Shabanowitz J, et al. Identification of the regulatory phosphorylation sites in pp42/mitogen-activated protein kinase (MAP kinase). EMBO J 1991 Apr;10(4):885-892.

(204) Yang SH, Sharrocks AD, Whitmarsh A. MAP kinase signalling cascades and transcriptional regulation. Gene 2012 Nov 1.

(205) Alessi DR, Gomez N, Moorhead G, Lewis T, Keyse SM, Cohen P. Inactivation of p42 MAP kinase by protein phosphatase $2 \mathrm{~A}$ and a protein tyrosine phosphatase, but not CL100, in various cell lines. Curr Biol 1995 Mar 1;5(3):283-295.

(206) Muda M, Theodosiou A, Rodrigues N, Boschert U, Camps M, Gillieron C, et al. The dual specificity phosphatases M3/6 and MKP-3 are highly selective for inactivation of distinct mitogen-activated protein kinases. J Biol Chem 1996 Nov 1;271(44):27205-27208.

(207) Buday L, Warne PH, Downward J. Downregulation of the Ras activation pathway by MAP kinase phosphorylation of Sos. Oncogene 1995 Oct 5;11(7):1327-1331.

(208) Li X, Huang Y, Jiang J, Frank SJ. ERK-dependent threonine phosphorylation of EGF receptor modulates receptor downregulation and signaling. Cell Signal 2008 Nov;20(11):2145-2155.

(209) Dougherty MK, Muller J, Ritt DA, Zhou M, Zhou XZ, Copeland TD, et al. Regulation of Raf-1 by direct feedback phosphorylation. Mol Cell 2005 Jan 21;17(2):215-224.

(210) Catalanotti F, Reyes G, Jesenberger V, Galabova-Kovacs G, de Matos Simoes R, Carugo O, et al. A Mek1-Mek2 heterodimer determines the strength and duration of the Erk signal. Nat Struct Mol Biol 2009 Mar;16(3):294-303.

(211) Ekerot M, Stavridis MP, Delavaine L, Mitchell MP, Staples C, Owens DM, et al. Negative-feedback regulation of FGF signalling by DUSP6/MKP-3 is driven by ERK1/2 and mediated by Ets factor binding to a conserved site within the DUSP6/MKP-3 gene promoter. Biochem J 2008 Jun 1;412(2):287-298.

(212) McCubrey JA, Steelman LS, Chappell WH, Abrams SL, Franklin RA, Montalto G, et al.

Ras/Raf/MEK/ERK and PI3K/PTEN/Akt/mTOR Cascade Inhibitors: How Mutations Can Result in Therapy Resistance and How to Overcome Resistance. Oncotarget 2012 Oct;3(10):1068-1111.

(213) McCubrey JA, Steelman LS, Chappell WH, Abrams SL, Montalto G, Cervello M, et al. Mutations and Deregulation of Ras/Raf/MEK/ERK and PI3K/PTEN/Akt/mTOR Cascades. Oncotarget 2012 Sep;3(9):954-987.

(214) Schlaepfer DD, Hanks SK, Hunter T, van der Geer P. Integrin-mediated signal transduction linked to Ras pathway by GRB2 binding to focal adhesion kinase. Nature 1994 Dec 22-29;372(6508):786-791.

(215) Pullikuth AK, Catling AD. Scaffold mediated regulation of MAPK signaling and cytoskeletal dynamics: a perspective. Cell Signal 2007 Aug;19(8):1621-1632. 
(216) Zou C, Luo Q, Qin J, Shi Y, Yang L, Ju B, et al. Osteopontin Promotes Mesenchymal Stem Cell Migration and Lessens Cell Stiffness via Integrin beta1, FAK, and ERK Pathways. Cell Biochem Biophys 2012 Oct 20.

(217) Kaomongkolgit R, Manokawinchoke J, Sanchavanakit N, Pavasant P, Sumrejkanchanakij P. Fibronectin supports TNF-alpha-induced osteopontin expression through beta1 integrin and ERK in $\mathrm{HN}$ 22 cells. Arch Oral Biol 2010 Feb;55(2):101-107.

(218) Puissant A, Dufies M, Fenouille N, Ben Sahra I, Jacquel A, Robert G, et al. Imatinib triggers mesenchymal-like conversion of CML cells associated with increased aggressiveness. $\mathrm{J} \mathrm{Mol} \mathrm{Cell} \mathrm{Biol}$ 2012 Aug;4(4):207-220.

(219) Albanell J, Codony-Servat J, Rojo F, Del Campo JM, Sauleda S, Anido J, et al. Activated extracellular signal-regulated kinases: association with epidermal growth factor receptor/transforming growth factor alpha expression in head and neck squamous carcinoma and inhibition by anti-epidermal growth factor receptor treatments. Cancer Res 2001 Sep 1;61(17):6500-6510.

(220) Santarpia L, Lippman SM, El-Naggar AK. Targeting the MAPK-RAS-RAF signaling pathway in cancer therapy. Expert Opin Ther Targets 2012 Jan;16(1):103-119.

(221) Adjei AA, Cohen RB, Franklin W, Morris C, Wilson D, Molina JR, et al. Phase I pharmacokinetic and pharmacodynamic study of the oral, small-molecule mitogen-activated protein kinase kinase 1/2 inhibitor AZD6244 (ARRY-142886) in patients with advanced cancers. J Clin Oncol 2008 May 1;26(13):21392146.

(222) Hainsworth JD, Cebotaru CL, Kanarev V, Ciuleanu TE, Damyanov D, Stella P, et al. A phase II, open-label, randomized study to assess the efficacy and safety of AZD6244 (ARRY-142886) versus pemetrexed in patients with non-small cell lung cancer who have failed one or two prior chemotherapeutic regimens. J Thorac Oncol 2010 Oct;5(10):1630-1636.

(223) Bennouna J, Lang I, Valladares-Ayerbes M, Boer K, Adenis A, Escudero P, et al. A Phase II, openlabel, randomised study to assess the efficacy and safety of the MEK1/2 inhibitor AZD6244 (ARRY142886) versus capecitabine monotherapy in patients with colorectal cancer who have failed one or two prior chemotherapeutic regimens. Invest New Drugs 2011 Oct;29(5):1021-1028.

(224) Wilhelm SM, Carter C, Tang L, Wilkie D, McNabola A, Rong H, et al. BAY 43-9006 exhibits broad spectrum oral antitumor activity and targets the RAF/MEK/ERK pathway and receptor tyrosine kinases involved in tumor progression and angiogenesis. Cancer Res 2004 Oct 1;64(19):7099-7109.

(225) Keating GM, Santoro A. Sorafenib: a review of its use in advanced hepatocellular carcinoma. Drugs 2009;69(2):223-240.

(226) Rous P. A Sarcoma of the Fowl Transmissible by an Agent Separable from the Tumor Cells. J Exp Med 1911 Apr 1;13(4):397-411.

(227) Stehelin D, Varmus HE, Bishop JM, Vogt PK. DNA related to the transforming gene(s) of avian sarcoma viruses is present in normal avian DNA. Nature 1976 Mar 11;260(5547):170-173.

(228) Kim LC, Song L, Haura EB. Src kinases as therapeutic targets for cancer. Nat Rev Clin Oncol 2009 Oct;6(10):587-595.

(229) Guarino M. Src signaling in cancer invasion. J Cell Physiol 2010 Apr;223(1):14-26. 
(230) Xu W, Harrison SC, Eck MJ. Three-dimensional structure of the tyrosine kinase c-Src. Nature 1997 Feb 13;385(6617):595-602.

(231) Krueger JG, Garber EA, Goldberg AR, Hanafusa H. Changes in amino-terminal sequences of pp60src lead to decreased membrane association and decreased in vivo tumorigenicity. Cell 1982 Apr;28(4):889-896.

(232) Sefton BM, Trowbridge IS, Cooper JA, Scolnick EM. The transforming proteins of Rous sarcoma virus, Harvey sarcoma virus and Abelson virus contain tightly bound lipid. Cell 1982 Dec;31(2 Pt 1):465474.

(233) Waksman G, Shoelson SE, Pant N, Cowburn D, Kuriyan J. Binding of a high affinity phosphotyrosyl peptide to the $\mathrm{Src} \mathrm{SH} 2$ domain: crystal structures of the complexed and peptide-free forms. Cell $1993 \mathrm{Mar}$ 12;72(5):779-790.

(234) Chatzizacharias NA, Kouraklis GP, Giaginis CT, Theocharis SE. Clinical significance of Src expression and activity in human neoplasia. Histol Histopathol 2012 Jun;27(6):677-692.

(235) Masaki T, Okada M, Tokuda M, Shiratori Y, Hatase O, Shirai M, et al. Reduced C-terminal Src kinase (Csk) activities in hepatocellular carcinoma. Hepatology 1999 Feb;29(2):379-384.

(236) Cooper JA, Gould KL, Cartwright CA, Hunter T. Tyr527 is phosphorylated in pp60c-src: implications for regulation. Science 1986 Mar 21;231(4744):1431-1434.

(237) Yamaguchi $\mathrm{H}$, Hendrickson WA. Structural basis for activation of human lymphocyte kinase Lck upon tyrosine phosphorylation. Nature 1996 Dec 5;384(6608):484-489.

(238) Cooper JA, King CS. Dephosphorylation or antibody binding to the carboxy terminus stimulates pp60c-src. Mol Cell Biol 1986 Dec;6(12):4467-4477.

(239) Zheng XM, Wang Y, Pallen CJ. Cell transformation and activation of pp60c-src by overexpression of a protein tyrosine phosphatase. Nature 1992 Sep 24;359(6393):336-339.

(240) Bjorge JD, Pang A, Fujita DJ. Identification of protein-tyrosine phosphatase 1B as the major tyrosine phosphatase activity capable of dephosphorylating and activating $\mathrm{C}$-Src in several human breast cancer cell lines. J Biol Chem 2000 Dec 29;275(52):41439-41446.

(241) Jung EJ, Kim CW. Interaction between chicken protein tyrosine phosphatase 1 (CPTP1)-like rat protein phosphatase 1 (PTP1) and p60(v-src) in v-src-transformed Rat-1 fibroblasts. Exp Mol Med 2002 Dec 31;34(6):476-480.

(242) Schaller MD, Hildebrand JD, Shannon JD, Fox JW, Vines RR, Parsons JT. Autophosphorylation of the focal adhesion kinase, pp125FAK, directs SH2-dependent binding of pp60src. Mol Cell Biol 1994 Mar;14(3):1680-1688.

(243) Luttrell DK, Lee A, Lansing TJ, Crosby RM, Jung KD, Willard D, et al. Involvement of pp60c-src with two major signaling pathways in human breast cancer. Proc Natl Acad Sci U S A 1994 Jan 4;91(1):83-87.

(244) Mao W, Irby R, Coppola D, Fu L, Wloch M, Turner J, et al. Activation of c-Src by receptor tyrosine kinases in human colon cancer cells with high metastatic potential. Oncogene 1997 Dec 18;15(25):30833090. 
(245) Tice DA, Biscardi JS, Nickles AL, Parsons SJ. Mechanism of biological synergy between cellular Src and epidermal growth factor receptor. Proc Natl Acad Sci U S A 1999 Feb 16;96(4):1415-1420.

(246) Courtneidge SA, Fumagalli S, Koegl M, Superti-Furga G, Twamley-Stein GM. The Src family of protein tyrosine kinases: regulation and functions. Dev Suppl 1993:57-64.

(247) Landgren E, Blume-Jensen P, Courtneidge SA, Claesson-Welsh L. Fibroblast growth factor receptor-1 regulation of Src family kinases. Oncogene 1995 May 18;10(10):2027-2035.

(248) LaVallee TM, Prudovsky IA, McMahon GA, Hu X, Maciag T. Activation of the MAP kinase pathway by FGF-1 correlates with cell proliferation induction while activation of the Src pathway correlates with migration. J Cell Biol 1998 Jun 29;141(7):1647-1658.

(249) Muthuswamy SK, Siegel PM, Dankort DL, Webster MA, Muller WJ. Mammary tumors expressing the neu proto-oncogene possess elevated c-Src tyrosine kinase activity. Mol Cell Biol 1994 Jan;14(1):735-743.

(250) Rahimi N, Hung W, Tremblay E, Saulnier R, Elliott B. c-Src kinase activity is required for hepatocyte growth factor-induced motility and anchorage-independent growth of mammary carcinoma cells. J Biol Chem 1998 Dec 11;273(50):33714-33721.

(251) Yeo MG, Partridge MA, Ezratty EJ, Shen Q, Gundersen GG, Marcantonio EE. Src SH2 arginine 175 is required for cell motility: specific focal adhesion kinase targeting and focal adhesion assembly function. Mol Cell Biol 2006 Jun;26(12):4399-4409.

(252) Thomas JW, Ellis B, Boerner RJ, Knight WB, White GC,2nd, Schaller MD. SH2- and SH3-mediated interactions between focal adhesion kinase and Src. J Biol Chem 1998 Jan 2;273(1):577-583.

(253) Thomas JW, Cooley MA, Broome JM, Salgia R, Griffin JD, Lombardo CR, et al. The role of focal adhesion kinase binding in the regulation of tyrosine phosphorylation of paxillin. J Biol Chem $1999 \mathrm{Dec}$ 17;274(51):36684-36692.

(254) Hamasaki K, Mimura T, Morino N, Furuya H, Nakamoto T, Aizawa S, et al. Src kinase plays an essential role in integrin-mediated tyrosine phosphorylation of Crk-associated substrate p130Cas. Biochem Biophys Res Commun 1996 May 15;222(2):338-343.

(255) Noren NK, Arthur WT, Burridge K. Cadherin engagement inhibits RhoA via p190RhoGAP. J Biol Chem 2003 Apr 18;278(16):13615-13618.

(256) Arthur WT, Petch LA, Burridge K. Integrin engagement suppresses RhoA activity via a c-Srcdependent mechanism. Curr Biol 2000 Jun 15;10(12):719-722.

(257) Buchsbaum RJ. Rho activation at a glance. J Cell Sci 2007 Apr 1;120(Pt 7):1149-1152.

(258) Webb DJ, Donais K, Whitmore LA, Thomas SM, Turner CE, Parsons JT, et al. FAK-Src signalling through paxillin, ERK and MLCK regulates adhesion disassembly. Nat Cell Biol 2004 Feb;6(2):154-161.

(259) Klemke RL, Cai S, Giannini AL, Gallagher PJ, de Lanerolle P, Cheresh DA. Regulation of cell motility by mitogen-activated protein kinase. J Cell Biol 1997 Apr 21;137(2):481-492. 
(260) Hashimoto S, Hirose M, Hashimoto A, Morishige M, Yamada A, Hosaka H, et al. Targeting AMAP1 and cortactin binding bearing an atypical src homology 3/proline interface for prevention of breast cancer invasion and metastasis. Proc Natl Acad Sci U S A 2006 May 2;103(18):7036-7041.

(261) Sabe H, Onodera Y, Mazaki Y, Hashimoto S. ArfGAP family proteins in cell adhesion, migration and tumor invasion. Curr Opin Cell Biol 2006 Oct;18(5):558-564.

(262) Hashimoto S, Onodera Y, Hashimoto A, Tanaka M, Hamaguchi M, Yamada A, et al. Requirement for Arf6 in breast cancer invasive activities. Proc Natl Acad Sci U S A 2004 Apr 27;101(17):6647-6652.

(263) Nam JM, Onodera Y, Mazaki Y, Miyoshi H, Hashimoto S, Sabe H. CIN85, a Cbl-interacting protein, is a component of AMAP1-mediated breast cancer invasion machinery. EMBO J 2007 Feb 7;26(3):647656.

(264) Courtneidge SA. Cell migration and invasion in human disease: the Tks adaptor proteins. Biochem Soc Trans 2012 Feb;40(1):129-132.

(265) Diaz B, Courtneidge SA. Redox signaling at invasive microdomains in cancer cells. Free Radic Biol Med 2012 Jan 15;52(2):247-256.

(266) Buschman MD, Bromann PA, Cejudo-Martin P, Wen F, Pass I, Courtneidge SA. The novel adaptor protein Tks4 (SH3PXD2B) is required for functional podosome formation. Mol Biol Cell 2009 Mar;20(5):1302-1311.

(267) Diaz B, Shani G, Pass I, Anderson D, Quintavalle M, Courtneidge SA. Tks5-dependent, noxmediated generation of reactive oxygen species is necessary for invadopodia formation. Sci Signal 2009 Sep 15;2(88):ra53.

(268) Seals DF, Azucena EF,Jr, Pass I, Tesfay L, Gordon R, Woodrow M, et al. The adaptor protein Tks5/Fish is required for podosome formation and function, and for the protease-driven invasion of cancer cells. Cancer Cell 2005 Feb;7(2):155-165.

(269) Lai SY, Johnson FM. Defining the role of the JAK-STAT pathway in head and neck and thoracic malignancies: implications for future therapeutic approaches. Drug Resist Updat 2010 Jun;13(3):67-78.

(270) Rivat C, Le Floch N, Sabbah M, Teyrol I, Redeuilh G, Bruyneel E, et al. Synergistic cooperation between the AP-1 and LEF-1 transcription factors in activation of the matrilysin promoter by the src oncogene: implications in cellular invasion. FASEB J 2003 Sep;17(12):1721-1723.

(271) Hsia DA, Mitra SK, Hauck CR, Streblow DN, Nelson JA, llic D, et al. Differential regulation of cell motility and invasion by FAK. J Cell Biol 2003 Mar 3;160(5):753-767.

(272) Hu J, Mukhopadhyay A, Truesdell P, Chander H, Mukhopadhyay UK, Mak AS, et al. Cdc42interacting protein 4 is a Src substrate that regulates invadopodia and invasiveness of breast tumors by promoting MT1-MMP endocytosis. J Cell Sci 2011 May 15;124(Pt 10):1739-1751.

(273) Wu X, Gan B, Yoo Y, Guan JL. FAK-mediated src phosphorylation of endophilin A2 inhibits endocytosis of MT1-MMP and promotes ECM degradation. Dev Cell 2005 Aug;9(2):185-196.

(274) Jiang A, Lehti K, Wang X, Weiss SJ, Keski-Oja J, Pei D. Regulation of membrane-type matrix metalloproteinase 1 activity by dynamin-mediated endocytosis. Proc Natl Acad Sci U S A 2001 Nov 20;98(24):13693-13698. 
(275) Baldassarre M, Pompeo A, Beznoussenko G, Castaldi C, Cortellino S, McNiven MA, et al. Dynamin participates in focal extracellular matrix degradation by invasive cells. Mol Biol Cell 2003 Mar;14(3):10741084.

(276) Ahn S, Maudsley S, Luttrell LM, Lefkowitz RJ, Daaka Y. Src-mediated tyrosine phosphorylation of dynamin is required for beta2-adrenergic receptor internalization and mitogen-activated protein kinase signaling. J Biol Chem 1999 Jan 15;274(3):1185-1188.

(277) Ochoa GC, Slepnev VI, Neff L, Ringstad N, Takei K, Daniell L, et al. A functional link between dynamin and the actin cytoskeleton at podosomes. J Cell Biol 2000 Jul 24;150(2):377-389.

(278) Vindis C, Teli T, Cerretti DP, Turner CE, Huynh-Do U. EphB1-mediated cell migration requires the phosphorylation of paxillin at Tyr-31/Tyr-118. J Biol Chem 2004 Jul 2;279(27):27965-27970.

(279) Badowski C, Pawlak G, Grichine A, Chabadel A, Oddou C, Jurdic P, et al. Paxillin phosphorylation controls invadopodia/podosomes spatiotemporal organization. Mol Biol Cell 2008 Feb;19(2):633-645.

(280) Calle Y, Carragher NO, Thrasher AJ, Jones GE. Inhibition of calpain stabilises podosomes and impairs dendritic cell motility. J Cell Sci 2006 Jun 1;119(Pt 11):2375-2385.

(281) Cortesio CL, Chan KT, Perrin BJ, Burton NO, Zhang S, Zhang ZY, et al. Calpain 2 and PTP1B function in a novel pathway with Src to regulate invadopodia dynamics and breast cancer cell invasion. $J$ Cell Biol 2008 Mar 10;180(5):957-971.

(282) van Oijen MG, Rijksen G, ten Broek FW, Slootweg PJ. Overexpression of c-Src in areas of hyperproliferation in head and neck cancer, premalignant lesions and benign mucosal disorders. J Oral Pathol Med 1998 Apr;27(4):147-152.

(283) Aleshin A, Finn RS. SRC: a century of science brought to the clinic. Neoplasia 2010 Aug;12(8):599607.

(284) Dittmann K, Mayer C, Kehlbach R, Rodemann HP. Radiation-induced caveolin-1 associated EGFR internalization is linked with nuclear EGFR transport and activation of DNA-PK. Mol Cancer 2008 Sep 12;7:69.

(285) Elsberger B, Stewart B, Tatarov O, Edwards J. Is Src a viable target for treating solid tumours? Curr Cancer Drug Targets 2010 Nov;10(7):683-694.

(286) Brooks HD, Glisson BS, Bekele BN, Johnson FM, Ginsberg LE, El-Naggar A, et al. Phase 2 study of dasatinib in the treatment of head and neck squamous cell carcinoma. Cancer 2011 May 15;117(10):2112-2119.

(287) Johnson FM, Saigal B, Talpaz M, Donato NJ. Dasatinib (BMS-354825) tyrosine kinase inhibitor suppresses invasion and induces cell cycle arrest and apoptosis of head and neck squamous cell carcinoma and non-small cell lung cancer cells. Clin Cancer Res 2005 Oct 1;11(19 Pt 1):6924-6932.

(288) Dai Y, Siemann DW. BMS-777607, a small-molecule met kinase inhibitor, suppresses hepatocyte growth factor-stimulated prostate cancer metastatic phenotype in vitro. Mol Cancer Ther 2010 Jun;9(6):1554-1561.

(289) Rice L, Lepler S, Pampo C, Siemann DW. Impact of the SRC inhibitor dasatinib on the metastatic phenotype of human prostate cancer cells. Clin Exp Metastasis 2012 Feb;29(2):133-142. 
(290) Sanchez-Bailon MP, Calcabrini A, Gomez-Dominguez D, Morte B, Martin-Forero E, Gomez-Lopez $\mathrm{G}$, et al. Src kinases catalytic activity regulates proliferation, migration and invasiveness of MDA-MB-231 breast cancer cells. Cell Signal 2012 Jun;24(6):1276-1286.

(291) Pichot CS, Hartig SM, Xia L, Arvanitis C, Monisvais D, Lee FY, et al. Dasatinib synergizes with doxorubicin to block growth, migration, and invasion of breast cancer cells. Br J Cancer $2009 \mathrm{Jul}$ 7;101(1):38-47.

(292) Nautiyal J, Majumder P, Patel BB, Lee FY, Majumdar AP. Src inhibitor dasatinib inhibits growth of breast cancer cells by modulating EGFR signaling. Cancer Lett 2009 Oct 8;283(2):143-151.

(293) Mayer EL, Baurain JF, Sparano J, Strauss L, Campone M, Fumoleau P, et al. A phase 2 trial of dasatinib in patients with advanced HER2-positive and/or hormone receptor-positive breast cancer. Clin Cancer Res 2011 Nov 1;17(21):6897-6904.

(294) Finn RS, Bengala C, Ibrahim N, Roche H, Sparano J, Strauss LC, et al. Dasatinib as a single agent in triple-negative breast cancer: results of an open-label phase 2 study. Clin Cancer Res 2011 Nov 1;17(21):6905-6913.

(295) Herold Cl, Chadaram V, Peterson BL, Marcom PK, Hopkins J, Kimmick GG, et al. Phase II trial of dasatinib in patients with metastatic breast cancer using real-time pharmacodynamic tissue biomarkers of Src inhibition to escalate dosing. Clin Cancer Res 2011 Sep 15;17(18):6061-6070.

(296) Koppikar P, Choi SH, Egloff AM, Cai Q, Suzuki S, Freilino M, et al. Combined inhibition of c-Src and epidermal growth factor receptor abrogates growth and invasion of head and neck squamous cell carcinoma. Clin Cancer Res 2008 Jul 1;14(13):4284-4291.

(297) Ammer AG, Kelley LC, Hayes KE, Evans JV, Lopez-Skinner LA, Martin KH, et al. Saracatinib Impairs Head and Neck Squamous Cell Carcinoma Invasion by Disrupting Invadopodia Function. J Cancer Sci Ther 2009 Nov 30;1(2):52-61.

(298) Rajeshkumar NV, Tan AC, De Oliveira E, Womack C, Wombwell H, Morgan S, et al. Antitumor effects and biomarkers of activity of AZD0530, a Src inhibitor, in pancreatic cancer. Clin Cancer Res 2009 Jun 15;15(12):4138-4146.

(299) Chang YM, Bai L, Liu S, Yang JC, Kung HJ, Evans CP. Src family kinase oncogenic potential and pathways in prostate cancer as revealed by AZD0530. Oncogene 2008 Oct 23;27(49):6365-6375.

(300) Gangadhar TC, Clark JI, Karrison T, Gajewski TF. Phase II study of the Src kinase inhibitor saracatinib (AZD0530) in metastatic melanoma. Invest New Drugs 2012 Nov 15.

(301) Kruh GD, Perego R, Miki T, Aaronson SA. The complete coding sequence of arg defines the Abelson subfamily of cytoplasmic tyrosine kinases. Proc Natl Acad Sci U S A 1990 Aug;87(15):58025806.

(302) Hantschel O, Nagar B, Guettler S, Kretzschmar J, Dorey K, Kuriyan J, et al. A myristoyl/phosphotyrosine switch regulates c-Abl. Cell 2003 Mar 21;112(6):845-857.

(303) Pluk H, Dorey K, Superti-Furga G. Autoinhibition of c-Abl. Cell 2002 Jan 25;108(2):247-259. 
(304) Nagar B, Hantschel O, Seeliger M, Davies JM, Weis WI, Superti-Furga G, et al. Organization of the SH3-SH2 unit in active and inactive forms of the c-Abl tyrosine kinase. Mol Cell 2006 Mar 17;21(6):787798.

(305) Cujec TP, Medeiros PF, Hammond P, Rise C, Kreider BL. Selection of v-abl tyrosine kinase substrate sequences from randomized peptide and cellular proteomic libraries using mRNA display. Chem Biol 2002 Feb;9(2):253-264.

(306) Colicelli J. ABL tyrosine kinases: evolution of function, regulation, and specificity. Sci Signal 2010 Sep 14;3(139):re6.

(307) Antoku S, Saksela K, Rivera GM, Mayer BJ. A crucial role in cell spreading for the interaction of Abl PxxP motifs with Crk and Nck adaptors. J Cell Sci 2008 Sep 15;121(Pt 18):3071-3082.

(308) Lewis JM, Baskaran R, Taagepera S, Schwartz MA, Wang JY. Integrin regulation of c-Abl tyrosine kinase activity and cytoplasmic-nuclear transport. Proc Natl Acad Sci U S A 1996 Dec 24;93(26):1517415179.

(309) McWhirter JR, Wang JY. An actin-binding function contributes to transformation by the Bcr-Abl oncoprotein of Philadelphia chromosome-positive human leukemias. EMBO J 1993 Apr;12(4):1533-1546.

(310) Taagepera S, McDonald D, Loeb JE, Whitaker LL, McElroy AK, Wang JY, et al. Nuclearcytoplasmic shuttling of C-ABL tyrosine kinase. Proc Natl Acad Sci U S A 1998 Jun 23;95(13):7457-7462.

(311) Van Etten RA, Jackson PK, Baltimore D, Sanders MC, Matsudaira PT, Janmey PA. The COOH terminus of the c-Abl tyrosine kinase contains distinct F- and G-actin binding domains with bundling activity. J Cell Biol 1994 Feb;124(3):325-340.

(312) Miao YJ, Wang JY. Binding of A/T-rich DNA by three high mobility group-like domains in c-Abl tyrosine kinase. J Biol Chem 1996 Sep 13;271(37):22823-22830.

(313) Miller AL, Wang Y, Mooseker MS, Koleske AJ. The Abl-related gene (Arg) requires its F-actinmicrotubule cross-linking activity to regulate lamellipodial dynamics during fibroblast adhesion. J Cell Biol 2004 May 10;165(3):407-419.

(314) Wang Y, Miller AL, Mooseker MS, Koleske AJ. The Abl-related gene (Arg) nonreceptor tyrosine kinase uses two F-actin-binding domains to bundle F-actin. Proc Natl Acad Sci U S A 2001 Dec 18;98(26):14865-14870.

(315) Bradley WD, Koleske AJ. Regulation of cell migration and morphogenesis by Abl-family kinases: emerging mechanisms and physiological contexts. J Cell Sci 2009 Oct 1;122(Pt 19):3441-3454.

(316) Maiani E, Diederich M, Gonfloni S. DNA damage response: the emerging role of c-Abl as a regulatory switch? Biochem Pharmacol 2011 Nov 15;82(10):1269-1276.

(317) Nagar B, Hantschel O, Young MA, Scheffzek K, Veach D, Bornmann W, et al. Structural basis for the autoinhibition of c-Abl tyrosine kinase. Cell 2003 Mar 21;112(6):859-871.

(318) Woodring PJ, Litwack ED, O'Leary DD, Lucero GR, Wang JY, Hunter T. Modulation of the F-actin cytoskeleton by c-Abl tyrosine kinase in cell spreading and neurite extension. J Cell Biol 2002 Mar 4;156(5):879-892. 
(319) Brasher BB, Van Etten RA. c-Abl has high intrinsic tyrosine kinase activity that is stimulated by mutation of the Src homology 3 domain and by autophosphorylation at two distinct regulatory tyrosines. $J$ Biol Chem 2000 Nov 10;275(45):35631-35637.

(320) Tanis KQ, Veach D, Duewel HS, Bornmann WG, Koleske AJ. Two distinct phosphorylation pathways have additive effects on Abl family kinase activation. Mol Cell Biol 2003 Jun;23(11):3884-3896.

(321) Sun X, Majumder P, Shioya H, Wu F, Kumar S, Weichselbaum R, et al. Activation of the cytoplasmic c-Abl tyrosine kinase by reactive oxygen species. J Biol Chem 2000 Jun 9;275(23):1723717240.

(322) Kharbanda S, Ren R, Pandey P, Shafman TD, Feller SM, Weichselbaum RR, et al. Activation of the C-Abl tyrosine kinase in the stress response to DNA-damaging agents. Nature 1995 Aug 31;376(6543):785-788.

(323) Burton EA, Oliver TN, Pendergast AM. Abl kinases regulate actin comet tail elongation via an NWASP-dependent pathway. Mol Cell Biol 2005 Oct;25(20):8834-8843.

(324) Smith-Pearson PS, Greuber EK, Yogalingam G, Pendergast AM. Abl kinases are required for invadopodia formation and chemokine-induced invasion. J Biol Chem 2010 Dec 17;285(51):40201-40211.

(325) Ganguly SS, Fiore LS, Sims JT, Friend JW, Srinivasan D, Thacker MA, et al. c-Abl and Arg are activated in human primary melanomas, promote melanoma cell invasion via distinct pathways, and drive metastatic progression. Oncogene 2011 Sep 5.

(326) Michael M, Vehlow A, Navarro C, Krause M. c-Abl, Lamellipodin, and Ena/VASP proteins cooperate in dorsal ruffling of fibroblasts and axonal morphogenesis. Curr Biol 2010 May 11;20(9):783-791.

(327) Comer AR, Ahern-Djamali SM, Juang JL, Jackson PD, Hoffmann FM. Phosphorylation of Enabled by the Drosophila Abelson tyrosine kinase regulates the in vivo function and protein-protein interactions of Enabled. Mol Cell Biol 1998 Jan;18(1):152-160.

(328) Bradley WD, Hernandez SE, Settleman J, Koleske AJ. Integrin signaling through Arg activates p190RhoGAP by promoting its binding to p120RasGAP and recruitment to the membrane. Mol Biol Cell 2006 Nov;17(11):4827-4836.

(329) Hernandez SE, Settleman J, Koleske AJ. Adhesion-dependent regulation of p190RhoGAP in the developing brain by the Abl-related gene tyrosine kinase. Curr Biol 2004 Apr 20;14(8):691-696.

(330) Sini P, Cannas A, Koleske AJ, Di Fiore PP, Scita G. Abl-dependent tyrosine phosphorylation of Sos-1 mediates growth-factor-induced Rac activation. Nat Cell Biol 2004 Mar;6(3):268-274.

(331) Boureux A, Furstoss O, Simon V, Roche S. Abl tyrosine kinase regulates a Rac/JNK and a Rac/Nox pathway for DNA synthesis and Myc expression induced by growth factors. J Cell Sci 2005 Aug 15;118(Pt 16):3717-3726.

(332) Kain KH, Klemke RL. Inhibition of cell migration by Abl family tyrosine kinases through uncoupling of Crk-CAS complexes. J Biol Chem 2001 May 11;276(19):16185-16192.

(333) Feller SM, Knudsen B, Hanafusa H. c-Abl kinase regulates the protein binding activity of c-Crk. EMBO J 1994 May 15;13(10):2341-2351. 
(334) Vuori K, Hirai H, Aizawa S, Ruoslahti E. Introduction of p130cas signaling complex formation upon integrin-mediated cell adhesion: a role for Src family kinases. Mol Cell Biol 1996 Jun;16(6):2606-2613.

(335) Turner CE. Paxillin and focal adhesion signalling. Nat Cell Biol 2000 Dec;2(12):E231-6.

(336) Lewis JM, Schwartz MA. Integrins regulate the association and phosphorylation of paxillin by c-Abl. J Biol Chem 1998 Jun 5;273(23):14225-14230.

(337) Ren R, Ye ZS, Baltimore D. Abl protein-tyrosine kinase selects the Crk adapter as a substrate using SH3-binding sites. Genes Dev 1994 Apr 1;8(7):783-795.

(338) Cao C, Ren X, Kharbanda S, Koleske A, Prasad KV, Kufe D. The ARG tyrosine kinase interacts with Siva-1 in the apoptotic response to oxidative stress. J Biol Chem 2001 Apr 13;276(15):11465-11468.

(339) Sun X, Wu F, Datta R, Kharbanda S, Kufe D. Interaction between protein kinase C delta and the cAbl tyrosine kinase in the cellular response to oxidative stress. J Biol Chem 2000 Mar 17;275(11):74707473.

(340) Baskaran R, Wood LD, Whitaker LL, Canman CE, Morgan SE, Xu Y, et al. Ataxia telangiectasia mutant protein activates C-Abl tyrosine kinase in response to ionizing radiation. Nature 1997 May 29;387(6632):516-519.

(341) Shafman T, Khanna KK, Kedar P, Spring K, Kozlov S, Yen T, et al. Interaction between ATM protein and c-Abl in response to DNA damage. Nature 1997 May 29;387(6632):520-523.

(342) Wen ST, Jackson PK, Van Etten RA. The cytostatic function of c-Abl is controlled by multiple nuclear localization signals and requires the p53 and Rb tumor suppressor gene products. EMBO J 1996 Apr 1;15(7):1583-1595.

(343) Truong T, Sun G, Doorly M, Wang JY, Schwartz MA. Modulation of DNA damage-induced apoptosis by cell adhesion is independently mediated by p53 and c-Abl. Proc Natl Acad Sci U S A 2003 Sep 2;100(18):10281-10286.

(344) Goldberg Z, Vogt Sionov R, Berger M, Zwang Y, Perets R, Van Etten RA, et al. Tyrosine phosphorylation of Mdm2 by c-Abl: implications for p53 regulation. EMBO J 2002 Jul 15;21(14):37153727.

(345) Waning DL, Lehman JA, Batuello CN, Mayo LD. c-Abl phosphorylation of Mdm2 facilitates Mdm2Mdmx complex formation. J Biol Chem 2011 Jan 7;286(1):216-222.

(346) Yuan ZM, Huang Y, Ishiko T, Kharbanda S, Weichselbaum R, Kufe D. Regulation of DNA damageinduced apoptosis by the c-Abl tyrosine kinase. Proc Natl Acad Sci U S A 1997 Feb 18;94(4):1437-1440.

(347) Hu W, Feng Z, Levine AJ. The Regulation of Multiple p53 Stress Responses is Mediated through MDM2. Genes Cancer 2012 Mar;3(3-4):199-208.

(348) Wang X, Zeng L, Wang J, Chau JF, Lai KP, Jia D, et al. A positive role for C-Abl in Atm and Atr activation in DNA damage response. Cell Death Differ 2011 Jan;18(1):5-15.

(349) Wang H, Zhai L, Xu J, Joo HY, Jackson S, Erdjument-Bromage H, et al. Histone H3 and H4 ubiquitylation by the CUL4-DDB-ROC1 ubiquitin ligase facilitates cellular response to DNA damage. Mol Cell 2006 May 5;22(3):383-394. 
(350) Yoshida K, Komatsu K, Wang HG, Kufe D. c-Abl tyrosine kinase regulates the human Rad9 checkpoint protein in response to DNA damage. Mol Cell Biol 2002 May;22(10):3292-3300.

(351) Kharbanda S, Pandey P, Jin S, Inoue S, Bharti A, Yuan ZM, et al. Functional interaction between DNA-PK and c-Abl in response to DNA damage. Nature 1997 Apr 17;386(6626):732-735.

(352) Nagano K, Itagaki C, Izumi T, Nunomura K, Soda Y, Tani K, et al. Rb plays a role in survival of Abldependent human tumor cells as a downstream effector of Abl tyrosine kinase. Oncogene 2006 Jan 26;25(4):493-502.

(353) Gonfloni S. DNA damage stress response in germ cells: role of c-Abl and clinical implications. Oncogene 2010 Nov 25;29(47):6193-6202.

(354) Cong F, Spencer S, Cote JF, Wu Y, Tremblay ML, Lasky LA, et al. Cytoskeletal protein PSTPIP1 directs the PEST-type protein tyrosine phosphatase to the c-Abl kinase to mediate Abl dephosphorylation. Mol Cell 2000 Dec;6(6):1413-1423.

(355) Plattner R, Irvin BJ, Guo S, Blackburn K, Kazlauskas A, Abraham RT, et al. A new link between the c-Abl tyrosine kinase and phosphoinositide signalling through PLC-gamma1. Nat Cell Biol 2003

Apr;5(4):309-319.

(356) Echarri A, Pendergast AM. Activated c-Abl is degraded by the ubiquitin-dependent proteasome pathway. Curr Biol 2001 Nov 13;11(22):1759-1765.

(357) Soubeyran P, Barac A, Szymkiewicz I, Dikic I. Cbl-ArgBP2 complex mediates ubiquitination and degradation of c-Abl. Biochem J 2003 Feb 15;370(Pt 1):29-34.

(358) Podar K, Raab MS, Tonon G, Sattler M, Barila D, Zhang J, et al. Up-regulation of c-Jun inhibits proliferation and induces apoptosis via caspase-triggered c-Abl cleavage in human multiple myeloma. Cancer Res 2007 Feb 15;67(4):1680-1688.

(359) Ernst T, Hochhaus A. Chronic myeloid leukemia: clinical impact of BCR-ABL1 mutations and other lesions associated with disease progression. Semin Oncol 2012 Feb;39(1):58-66.

(360) Wang Y, Gu M, Mi Y, Qiu L, Bian S, Wang J. Clinical characteristics and outcomes of mixed phenotype acute leukemia with Philadelphia chromosome positive and/or bcr-abl positive in adult. Int $\mathrm{J}$ Hematol 2011 Dec;94(6):552-555.

(361) Srinivasan D, Plattner R. Activation of Abl tyrosine kinases promotes invasion of aggressive breast cancer cells. Cancer Res 2006 Jun 1;66(11):5648-5655.

(362) Srinivasan D, Sims JT, Plattner R. Aggressive breast cancer cells are dependent on activated Abl kinases for proliferation, anchorage-independent growth and survival. Oncogene $2008 \mathrm{Feb}$ 14;27(8):1095-1105.

(363) Lin J, Sun T, Ji L, Deng W, Roth J, Minna J, et al. Oncogenic activation of c-Abl in non-small cell lung cancer cells lacking FUS1 expression: inhibition of c-Abl by the tumor suppressor gene product Fus1. Oncogene 2007 Oct 25;26(49):6989-6996.

(364) Yanagawa T, Harada H, Iwasa S, Tabuchi K, Omura K, Suzuki H, et al. c-Abl expression in oral squamous cell carcinomas. Oral Oncol 2000 Jan;36(1):89-94. 
(365) Allington TM, Galliher-Beckley AJ, Schiemann WP. Activated Abl kinase inhibits oncogenic transforming growth factor-beta signaling and tumorigenesis in mammary tumors. FASEB J 2009 Dec;23(12):4231-4243.

(366) Frasca F, Vigneri P, Vella V, Vigneri R, Wang JY. Tyrosine kinase inhibitor STI571 enhances thyroid cancer cell motile response to Hepatocyte Growth Factor. Oncogene 2001 Jun 28;20(29):38453856.

(367) Druker BJ, Guilhot F, O'Brien SG, Gathmann I, Kantarjian H, Gattermann N, et al. Five-year followup of patients receiving imatinib for chronic myeloid leukemia. N Engl J Med 2006 Dec 7;355(23):24082417.

(368) Roychowdhury S, Talpaz M. Managing resistance in chronic myeloid leukemia. Blood Rev 2011 Nov;25(6):279-290.

(369) Ashman LK, Griffith R. Therapeutic targeting of c-KIT in cancer. Expert Opin Investig Drugs 2012 Nov 6.

(370) Tsao AS, Liu S, Fujimoto J, Wistuba II, Lee JJ, Marom EM, et al. Phase II trials of imatinib mesylate and docetaxel in patients with metastatic non-small cell lung cancer and head and neck squamous cell carcinoma. J Thorac Oncol 2011 Dec;6(12):2104-2111.

(371) Cristofanilli M, Morandi P, Krishnamurthy S, Reuben JM, Lee BN, Francis D, et al. Imatinib mesylate (Gleevec) in advanced breast cancer-expressing C-Kit or PDGFR-beta: clinical activity and biological correlations. Ann Oncol 2008 Oct;19(10):1713-1719.

(372) Modi S, Seidman AD, Dickler M, Moasser M, D'Andrea G, Moynahan ME, et al. A phase II trial of imatinib mesylate monotherapy in patients with metastatic breast cancer. Breast Cancer Res Treat 2005 Mar;90(2):157-163.

(373) Chew HK, Barlow WE, Albain K, Lew D, Gown A, Hayes DF, et al. A phase II study of imatinib mesylate and capecitabine in metastatic breast cancer: Southwest Oncology Group Study 0338. Clin Breast Cancer 2008 Dec;8(6):511-515.

(374) Lin AM, Rini BI, Weinberg V, Fong K, Ryan CJ, Rosenberg JE, et al. A phase II trial of imatinib mesylate in patients with biochemical relapse of prostate cancer after definitive local therapy. BJU Int 2006 Oct;98(4):763-769.

(375) Gharibo M, Patrick-Miller L, Zheng L, Guensch L, Juvidian P, Poplin E. A phase II trial of imatinib mesylate in patients with metastatic pancreatic cancer. Pancreas 2008 May;36(4):341-345.

(376) Chen J, Rocken C, Nitsche B, Hosius C, Gschaidmeier H, Kahl S, et al. The tyrosine kinase inhibitor imatinib fails to inhibit pancreatic cancer progression. Cancer Lett 2006 Feb 28;233(2):328-337.

(377) Ohoka Y, Takai Y. Isolation and characterization of cortactin isoforms and a novel cortactin-binding protein, CBP90. Genes Cells 1998 Sep;3(9):603-612.

(378) Katsube T, Takahisa M, Ueda R, Hashimoto N, Kobayashi M, Togashi S. Cortactin associates with the cell-cell junction protein ZO-1 in both Drosophila and mouse. J Biol Chem 1998 Nov 6;273(45):2967229677. 
(379) Hirakawa H, Shibata K, Nakayama T. Localization of cortactin is associated with colorectal cancer development. Int J Oncol 2009 Dec;35(6):1271-1276.

(380) Zhang S, Yu D. Targeting Src family kinases in anti-cancer therapies: turning promise into triumph. Trends Pharmacol Sci 2012 3;33(3):122-128.

(381) Shang X, Zhou YT, Low BC. Concerted regulation of cell dynamics by BNIP-2 and Cdc42GAP homology/Sec14p-like, proline-rich, and GTPase-activating protein domains of a novel Rho GTPaseactivating protein, BPGAP1. J Biol Chem 2003 Nov 14;278(46):45903-45914.

(382) Lua BL, Low BC. BPGAP1 interacts with cortactin and facilitates its translocation to cell periphery for enhanced cell migration. Mol Biol Cell 2004 Jun;15(6):2873-2883.

(383) Le Clainche C, Pauly BS, Zhang CX, Engqvist-Goldstein AE, Cunningham K, Drubin DG. A Hip1Rcortactin complex negatively regulates actin assembly associated with endocytosis. EMBO J $2007 \mathrm{Mar}$ 7;26(5):1199-1210.

(384) Tian L, Chen L, McClafferty H, Sailer CA, Ruth P, Knaus HG, et al. A noncanonical SH3 domain binding motif links BK channels to the actin cytoskeleton via the SH3 adapter cortactin. FASEB J 2006 Dec;20(14):2588-2590.

(385) Tian L, McClafferty H, Chen L, Shipston MJ. Reversible tyrosine protein phosphorylation regulates large conductance voltage- and calcium-activated potassium channels via cortactin. J Biol Chem 2008 Feb 8;283(6):3067-3076.

(386) Onodera Y, Hashimoto S, Hashimoto A, Morishige M, Mazaki Y, Yamada A, et al. Expression of AMAP1, an ArfGAP, provides novel targets to inhibit breast cancer invasive activities. EMBO J 2005 Mar 9;24(5):963-973.

(387) Randazzo PA, Andrade J, Miura K, Brown MT, Long YQ, Stauffer S, et al. The Arf GTPaseactivating protein ASAP1 regulates the actin cytoskeleton. Proc Natl Acad Sci U S A 2000 Apr 11;97(8):4011-4016.

(388) Inoue H, Ha VL, Prekeris R, Randazzo PA. Arf GTPase-activating protein ASAP1 interacts with Rab11 effector FIP3 and regulates pericentrosomal localization of transferrin receptor-positive recycling endosome. Mol Biol Cell 2008 Oct;19(10):4224-4237.

(389) Bharti S, Inoue H, Bharti K, Hirsch DS, Nie Z, Yoon HY, et al. Src-dependent phosphorylation of ASAP1 regulates podosomes. Mol Cell Biol 2007 Dec;27(23):8271-8283.

(390) Morishige M, Hashimoto S, Ogawa E, Toda Y, Kotani H, Hirose M, et al. GEP100 links epidermal growth factor receptor signalling to Arf6 activation to induce breast cancer invasion. Nat Cell Biol 2008 Jan;10(1):85-92.

(391) Kempiak SJ, Yamaguchi H, Sarmiento C, Sidani M, Ghosh M, Eddy RJ, et al. A neural WiskottAldrich Syndrome protein-mediated pathway for localized activation of actin polymerization that is regulated by cortactin. J Biol Chem 2005 Feb 18;280(7):5836-5842.

(392) Matas OB, Martinez-Menarguez JA, Egea G. Association of Cdc42/N-WASP/Arp2/3 signaling pathway with Golgi membranes. Traffic 2004 Nov;5(11):838-846. 
(393) Mizutani K, Miki H, He H, Maruta H, Takenawa T. Essential role of neural Wiskott-Aldrich syndrome protein in podosome formation and degradation of extracellular matrix in src-transformed fibroblasts. Cancer Res 2002 Feb 1;62(3):669-674.

(394) Henley JR, McNiven MA. Association of a dynamin-like protein with the Golgi apparatus in mammalian cells. J Cell Biol 1996 May;133(4):761-775.

(395) Jones SM, Howell KE, Henley JR, Cao H, McNiven MA. Role of dynamin in the formation of transport vesicles from the trans-Golgi network. Science 1998 Jan 23;279(5350):573-577.

(396) Cao H, Garcia F, McNiven MA. Differential distribution of dynamin isoforms in mammalian cells. Mol Biol Cell 1998 Sep;9(9):2595-2609.

(397) McNiven MA, Kim L, Krueger EW, Orth JD, Cao H, Wong TW. Regulated interactions between dynamin and the actin-binding protein cortactin modulate cell shape. J Cell Biol 2000 Oct 2;151(1):187198.

(398) Redecker P, Bockmann J, Bockers TM. Secretory granules of hypophyseal and pancreatic endocrine cells contain proteins of the neuronal postsynaptic density. Cell Tissue Res 2007 Apr;328(1):49-55.

(399) Naisbitt S, Kim E, Tu JC, Xiao B, Sala C, Valtschanoff J, et al. Shank, a novel family of postsynaptic density proteins that binds to the NMDA receptor/PSD-95/GKAP complex and cortactin. Neuron 1999 Jul;23(3):569-582.

(400) Du Y, Weed SA, Xiong WC, Marshall TD, Parsons JT. Identification of a novel cortactin SH3 domain-binding protein and its localization to growth cones of cultured neurons. Mol Cell Biol 1998 Oct;18(10):5838-5851.

(401) Hou P, Estrada L, Kinley AW, Parsons JT, Vojtek AB, Gorski JL. Fgd1, the Cdc42 GEF responsible for Faciogenital Dysplasia, directly interacts with cortactin and mAbp1 to modulate cell shape. Hum Mol Genet 2003 Aug 15;12(16):1981-1993.

(402) Estrada L, Caron E, Gorski JL. Fgd1, the Cdc42 guanine nucleotide exchange factor responsible for faciogenital dysplasia, is localized to the subcortical actin cytoskeleton and Golgi membrane. Hum Mol Genet 2001 Mar 1;10(5):485-495.

(403) Martinez-Quiles N, Rohatgi R, Anton IM, Medina M, Saville SP, Miki H, et al. WIP regulates NWASP-mediated actin polymerization and filopodium formation. Nat Cell Biol 2001 May;3(5):484-491.

(404) Dudek SM, Jacobson JR, Chiang ET, Birukov KG, Wang P, Zhan X, et al. Pulmonary endothelial cell barrier enhancement by sphingosine 1-phosphate: roles for cortactin and myosin light chain kinase. $J$ Biol Chem 2004 Jun 4;279(23):24692-24700.

(405) Dudek SM, Birukov KG, Zhan X, Garcia JG. Novel interaction of cortactin with endothelial cell myosin light chain kinase. Biochem Biophys Res Commun 2002 Nov 8;298(4):511-519.

(406) Zhao J, Singleton PA, Brown ME, Dudek SM, Garcia JG. Phosphotyrosine protein dynamics in cell membrane rafts of sphingosine-1-phosphate-stimulated human endothelium: role in barrier enhancement. Cell Signal 2009 Dec;21(12):1945-1960. 
(407) Garcia JG, Verin AD, Schaphorst K, Siddiqui R, Patterson CE, Csortos C, et al. Regulation of endothelial cell myosin light chain kinase by Rho, cortactin, and p60(src). Am J Physiol 1999 Jun;276(6 Pt 1):L989-98.

(408) Lin J, Liu J, Wang Y, Zhu J, Zhou K, Smith N, et al. Differential regulation of cortactin and N-WASPmediated actin polymerization by missing in metastasis (MIM) protein. Oncogene 2005 Mar 17;24(12):2059-2066.

(409) Gonzalez-Quevedo R, Shoffer M, Horng L, Oro AE. Receptor tyrosine phosphatase-dependent cytoskeletal remodeling by the hedgehog-responsive gene MIM/BEG4. J Cell Biol 2005 Jan 31;168(3):453-463.

(410) Bershteyn M, Atwood SX, Woo WM, Li M, Oro AE. MIM and cortactin antagonism regulates ciliogenesis and hedgehog signaling. Dev Cell 2010 Aug 17;19(2):270-283.

(411) Lynch DK, Winata SC, Lyons RJ, Hughes WE, Lehrbach GM, Wasinger V, et al. A Cortactin-CD2associated protein (CD2AP) complex provides a novel link between epidermal growth factor receptor endocytosis and the actin cytoskeleton. J Biol Chem 2003 Jun 13;278(24):21805-21813.

(412) Zhao J, Bruck S, Cemerski S, Zhang L, Butler B, Dani A, et al. CD2AP Links Cortactin and Capping Protein at the Cell Periphery to Facilitate Lamellipodia Formation. Mol Cell Biol 2012 Oct 22.

(413) Kelley LC, Weed SA. Cortactin is a substrate of activated Cdc42-associated kinase 1 (ACK1) during ligand-induced epidermal growth factor receptor downregulation. PLoS One 2012;7(8):e44363. 


\section{Study 1: Ableson Kinases Negatively Regulate Invadopodia Function and Invasion in Head and Neck Squamous Cell Carcinoma by Inhibiting an HB-EGF Autocrine Loop}

Karen E. Hayes, B.S., Elyse L. Walk, B.S., Amanda Gatesman Ammer, Ph.D., Laura C. Kelley ${ }^{1}$, Ph.D., Karen H. Martin, Ph.D. and Scott A. Weed", Ph.D.

Department of Neurobiology and Anatomy, Program in Cancer Cell Biology, Mary Babb Randolph Cancer Center, West Virginia University, Morgantown, West Virginia, 265069300, United States of America

${ }^{1}$ Present Address: Biology Department, Duke University, Durham, North Carolina, 27708, United States of America

Funding Support: National Institute of Health grants R01 DE014578, P20 RR16440 and the West Virginia University Mary Babb Randolph Cancer Center

Published in Oncogene 2012 Nov 12;1(2):adv. online pub. doi: 10.1038/onc.2012.513

Running title: Abl negatively regulates invadopodia function

"Corresponding author:

Scott A. Weed

West Virginia University

Mary Babb Randolph Cancer Center

Morgantown, WV 26506-9300

Phone: 304-293-3016

Fax: 304-293-4667

Email: scweed@hsc.wvu.edu

Keywords: Abl, imatinib mesylate, invadopodia, invasion, head and neck cancer, cortactin 


\section{Abstract}

Head and neck squamous cell carcinoma (HNSCC) has a proclivity for locoregional invasion. HNSCC mediates invasion in part through invadopodia-based proteolysis of the extracellular matrix (ECM). Activation of Src, Erk1/2, Abl and Arg downstream of epidermal growth factor receptor (EGFR) modulates invadopodia activity through phosphorylation of the actin regulatory protein cortactin. In MDA-MB-231 breast cancer cells, Abl and Arg function downstream of Src to phosphorylate cortactin, promoting invadopodia ECM degradation activity and thus assigning a pro-invasive role for Ableson kinases. We report that $\mathrm{Abl}$ kinases have an opposite, negative regulatory role in HNSCC where they suppress invadopodia and tumor invasion. Impairment of Abl expression or Abl kinase activity with imatinib mesylate enhanced HNSCC matrix degradation and $3 D$ collagen invasion, functions that were impaired in MDA-MB-231. HNSCC lines with elevated EGFR and Src activation did not contain increased Abl or Arg kinase activity, suggesting Src could bypass Abl/Arg to phosphorylate cortactin and promote invadopodia ECM degradation. Src transformed $\mathrm{Abl}^{-/ /} / \mathrm{Arg}^{-/-}$fibroblasts produced ECM degrading invadopodia containing pY421 cortactin, indicating that $\mathrm{Abl} / \mathrm{Arg}$ are dispensable for invadopodia function in this system. Imatinib treated HNSCC cells had increased EGFR, Erk1/2 and Src activation, enhancing cortactin pY421 and pS405/418 required for invadopodia function. Imatinib stimulated shedding of the EGFR ligand heparin-binding EGF-like growth factor (HB-EGF) from HNSCC cells, where soluble HB-EGF enhanced invadopodia ECM degradation in HNSCC but not in MDA-MB-231. HNSCC cells treated with inhibitors of the EGFR invadopodia pathway indicated that EGFR and $\mathrm{Src}$ are required for invadopodia function. Collectively our results indicate that Abl kinases negatively regulate HNSCC invasive processes through suppression of an HB-EGF autocrine loop responsible for activating a EGFR-Src-cortactin cascade, in contrast to the invasion promoting functions of $\mathrm{Abl}$ kinases in breast and other cancer types. Our results provide mechanistic support for recent failed HNSCC clinical trials utilizing imatinib. 


\section{Introduction}

HNSCC is an aggressive disease characterized by extensive locoregional invasion and cervical lymph node metastasis $(1,2)$. Overexpression of EGFR is common in HNSCC and correlates with enhanced invasion and nodal involvement (3-6). EGFR inhibition as adjuvant therapy in HNSCC increases survival, highlighting the importance of downstream EGFR signaling pathways in HNSCC progression (7). Downstream EGFR signaling cascades in HNSCC that promote invasion and metastasis utilize Src, Erk, $\mathrm{PI} 3$ kinase, Akt and STATs (8-11), supporting a role for EGFR-generated signals as important regulators of invasion promoting pathways in HNSCC.

Src kinase activation within the EGFR pathway is critical for driving tumor invasion $(12,13)$. Elevated Src expression and activity is frequently found in HNSCC and other tumor types, where it has become a focus for targeted therapeutic design $(14,15)$. While Src targeted drugs have been developed and demonstrate anti-invasive properties in preclinical studies (16-19), recent phase II trials demonstrate virtually no benefit for HNSCC patients with monotherapeutic Src inhibitor treatment $(19,20)$. While combination therapy with receptor tyrosine kinase inhibitors increases efficacy (21-23), a clearer mechanistic understanding of how Src-based signaling governs HNSCC invasion is needed for the development of improved therapeutic strategies.

In carcinomas, Src activation results in the formation of invadopodia, actin-rich membraneous protrusions responsible for extracellular matrix (ECM) proteolysis, allowing tumor cells to infiltrate the stroma and vasculature $(24,25)$. Src kinase activity regulates the cyclic development of non-degradative (pre-invadopodia) and active (mature) invadopodia $(24,25)$. Maturation of pre-invadopodia involves recruitment and activation of matrix metalloproteinase MMP-14 to initiate ECM degradation (26-28).

Invadopodia maturation also involves increased $\mathrm{F}$-actin polymerization responsible for driving plasma membrane protrusion $(24,25,29)$. A prominent component of the invadopodia F-actin core is cortactin, an F-actin binding protein that activates the actinrelated protein (Arp)2/3 complex to stimulate branched actin polymerization (30-33). Cortactin binds neuronal Wiskott-Aldrich Syndrome protein (N-WASp), a second 
activator of Arp2/3 complex following phosphorylation of cortactin S405 and S418 by Erk1/2 (33-35). Cortactin modulates shifting of pre- to mature invadopodia through phosphorylation of two Src-targeted tyrosine residues (Y421 and Y470 in humans) $(27,28,36)$. Y421 and Y470 phosphorylation results in SH2-dependent recruitment of the adaptor protein NCK1, which in turn binds and activates N-WASp to promote additional Arp2/3 activation (28,37-39). Along with tyrosine phosphorylation, cortactin domains that bind Arp2/3 and N-WASp are also required for invadopodia formation, collectively highlighting the importance of cortactin in invadopodia biogenesis and regulation $(28,40,41)$.

The Abelson kinases $\mathrm{Abl}$ and Arg regulate actin cytoskeletal remodeling during motility and invasion (42-44). While Abl regulation of leukemic tumorigenesis is well established $(45,46)$, Abl activity in solid tumors promotes multiple aspects of neoplastic progression, including increased invasion and metastasis (47-50). Activation of Abl and Arg downstream of EGFR and Src leads to direct cortactin phosphorylation at Y421 and Y470 responsible for invadopodia maturation in breast cancer and melanoma cell lines (51-55). In particular, Src-mediated activation of Arg and subsequent cortactin tyrosine phosphorylation has led to the proposal that Arg is the terminal kinase responsible for cortactin tyrosine phosphorylation required for invadopodia maturation $(51,56)$.

Since invasive HNSCC typically contains elevated EGFR and Src activity, we postulated that downstream activation of $\mathrm{Abl}$ kinases may regulate invadopodia through cortactin phosphorylation in this tumor type. Paradoxically, we show that elimination of Abl expression results in enhanced invadopodia-based gelantinase activity in multiple HNSCC cells lines but not in MDA-MB-231 cells. Inhibition of Abl family kinase activity with imatinib mesylate (STI571; Gleevac) in HNSCC cells resulted in enhanced invadopodia maturation and cell invasion, whereas these processes were impaired in MDA-MB-231. Analysis of EGFR signaling indicates that EGFR and Src are hyperactivated in HNSCC compared to MDA-MB-231 cells. Introduction of active Src into $\mathrm{Abl}^{-/} / \mathrm{Arg}^{-/}$cells induced invadopodia formation, ECM matrix degradation and cortactin tyrosine phosphorylation, suggesting that elevated Src activity can bypass the requirement for $\mathrm{Abl}$ or $\mathrm{Arg}$ in invadopodia maturation. Imatinib treatment of HNSCC 
cells resulted in dose-dependent activation of EGFR, $\operatorname{Src}$ and Erk1/2, resulting in elevated cortactin tyrosine and serine phosphorylation absent in treated MDA-MB-231 cells. Imatinib enhanced production and shedding of the EGFR ligand HB-EGF in HNSCC cells, where soluble HB-EGF stimulated HNSCC ECM degradation. Inhibition of Src and Abl kinases with the dual specificity drug saracatinib suppressed EGFR activation and ECM degradation in HNSCC, suggesting that Src is responsible for mediating the pro-invasive signals resultant from imatinib-mediated Abl family kinase inactivation. Our results indicate that in HNSCC Abl kinases serve to suppress invadopodia formation and tumor cell invasion by downregulating autocrine HB-EGF activation of the EGFR-Src-cortactin signaling pathway, in contrast to the pro-invasive function of $\mathrm{Abl}$ and $\mathrm{Arg}$ in breast and other solid tumors. These results suggest that $\mathrm{Abl}$ kinase function in cancer invasion is context dependent, providing molecular insight into the mechanism behind the recent failure of clinical trials with imatinib in HNSCC patients (57). 


\section{$\underline{\text { Results }}$}

\section{Abl expression suppresses invadopodia activity in HNSCC cells}

Since HNSCC cells form invadopodia $(36,58)$ and Abl kinases mediate invadopodia function in other tumor types $(51,53,55)$, we evaluated the role of Abl in HNSCC invadopodia formation and function. HA-tagged Abl localized within UMSCC1 invadopodia (Figure 1a) and in Src-expressing 1483 cells (Supplementary Figure 1a), implying a functional role. This was investigated by knockdown of Abl expression using RNA interference (RNAi). Stable UMSCC1 clones expressing an Abl-specific short hairpin RNA (shRNA2 and 3) reduced Abl expression by 65\% compared to controls (Ctl2 and 8) (Figure 1b). Expression of wild-type (WT) murine Abl in shRNA cells restored expression to endogenous levels (WT5 and 6). Abl knockdown resulted in a $44 \%$ increase in gelatin degradation compared to control (Ctl) and WT lines (Figure 1cd) without affecting the number of cells degrading ECM or the number of invadopodia per cell (Supplementary Figure 1b-c). Abl knockdown in MDA-MB-231 cells did not impact matrix proteolysis (Supplementary Figure 2a-c), in agreement with previous results (51). These data suggest that Abl expression negatively regulates invadopodia function in HNSCC cells, as opposed its invadopodia promoting role in other tumor cell types $(51,53,55)$.

\section{Imatinib treatment enhances HNSCC invadopodia activity}

To determine if Abl kinase activity was responsible for the negative regulatory effects on invadopodia function in HNSCC cells, OSC19 and UMSCC1 cells were treated with the Abl family kinase inhibitor imatinib mesylate. Analysis of Crk phosphorylation confirmed partial inhibition of Abl kinase activity in imatinib-treated lines (Figure 2a). Imatinib treatment resulted in a dose-dependent increase in OSC19 and UMSCC1 ECM degradation, demonstrating a net 2.5-3.0-fold enhancement observed at the highest tolerated concentration (15 $\mu \mathrm{M}$ ) (Figure 2b-c, 2e). In contrast, MDA-MB-231 cells treated with imatinib resulted in a $70 \%$ reduction in matrix proteolysis (Figure $2 \mathrm{~d}$-e). The effects on treated HNSCC and MDA-MB-231 cells is likely Abl family kinase specific, since the alternative imatinib target PDGFR is not expressed in these lines 
(Supplementary Figure 3). These data indicate that imatinib impairment of Abl family kinase activity in HNSCC relieves the inhibitory effect of Abl on invadopodia function, promoting ECM degradation.

\section{Imatinib treatment stimulates HNSCC invasion}

To determine if imatinib-enhanced HNSCC invadopodia activity corresponds with increased invasive behavior, 3D invasion assays were conducted using tumor spheroids embedded in collagen I. OSC19 and UMSCC1 cells treated with $10 \mu \mathrm{M}$ imatinib resulted in enhanced invasion, whereas invasion of imatinib-treated MDA-MB-231 cells was inhibited after $24 \mathrm{~h}$ (Figure 3a-c). Average invaded distances were increased by $100 \mu \mathrm{M}$ for OSC19 and $204 \mu \mathrm{M}$ for UMSCC 1 cells, while MDA-MB-231 invasion was suppressed by $47 \mu \mathrm{M}$ (Figure $3 \mathrm{~d}$ ). These results indicate that imatinib treatment has opposing effects on tumor cell invasion, enhancing HNSCC cell invasion while impairing the invasiveness of MDA-MB-231.

\section{Activated Src can bypass Abl and Arg to promote invadopodia formation}

To address the contrasting invasive roles of Abl family kinases in HNSCC and MDAMB-231 cells, protein levels and activation of known invadopodia kinases were evaluated. Western blotting demonstrated increased Abl expression in HNSCC lines compared to MDA-MB-231 cells, whereas Arg protein levels were equivalent (Figure 4a). In spite of increased Abl expression in HNSCC cells, Abl activation was not enhanced, as evidenced by similar Crk pY221 levels between HNSCC and MDA-MB231 cells. However, OSC19 and UMSCC1 consistently displayed increased EGFR and Src activity compared to MDA-MB-231 (Figure 4a).

The increased Src activation in HNSCC cells suggested that active Src might directly regulate invadopodia activity independent of $\mathrm{Abl}$ and $\mathrm{Arg}$. To test this, $\mathrm{Abl}^{-/ /} / \mathrm{Arg}^{-/-}$ mouse embryo fibroblasts (DKO) were used to simultaneously evaluate the role of $\mathrm{Abl}$ and Arg on invadopodia function. Lack of $\mathrm{Abl}$ and Arg expression in DKO cells was verified by immunoblotting (Supplementary Figure 4a). EGF stimulation of wild-type (WT) MEFs demonstrated a threefold increase in cortactin pY421 over non-stimulated controls, whereas stimulated DKO cells showed a 1.5 fold increase over basal levels 
(Figure $4 b$ ). While these results confirm that $\mathrm{Abl}$ and Arg contribute to cortactin tyrosine phosphorylation, they also indicate that additional cortactin tyrosine kinases are utilized downstream of EGFR. To determine if activated Src promotes invadopodia formation independent of $\mathrm{Abl}$ and Arg, WT and DKO cells transfected with activated Src (527F) were assayed for cortactin tyrosine phosphorylation, invadopodia formation and ECM proteolysis. DKO cells expressing 527F Src contained abundant invadopodia with pY421 cortactin that degraded ECM, similar to 527F Src-transformed WT cells (Figure 4c). The ability of DKO cells to degrade gelatin matrix was dependent on Src kinase activity, since kinase inactive Src (K295M) failed to promote matrix proteolysis (Figure $4 \mathrm{~d})$. Expression of $527 \mathrm{~F} \mathrm{Src}$ in DKO cells resulted in an $86 \%$ increase in matrix degradation area compared to $527 \mathrm{~F}$ Src-expressing WT cells (Figure 4d-e, Supplementary Figure 4b). The amount of gelatin degradation per cell area in DKO 527F Src cells was inhibited by $60 \%$ following re-expression of WT Abl (Figure $4 \mathrm{~d}-\mathrm{e}$ ), in agreement with increased matrix degradation by UMSCC1 cells with Abl knockdown (Figure 1c-d). Collectively these results indicate that Abl expression suppresses invadopodia activity in Src-transformed mouse embryo fibroblasts, and that Abl and Arg are not essential for cortactin tyrosine phosphorylation or invadopodia formation downstream of active Src.

\section{Imatinib treatment enhances activation of the EGFR-invadopodia signaling pathway in HNSCC}

To determine the basis for the differential regulation of invadopodia activity and invasion when Abl/Arg activity is suppressed in HNSCC and MDA-MB-231, cells treated with imatinib were evaluated for activation of EGFR and downstream invadopodia signaling components. Increased EGFR activation was observed in imatinib-treated OSC19 and UMSCC1 cells, whereas no increase was observed in MDA-MB-231 (Figure 5a, Supplementary Figure 5a). A corresponding activation pattern was found for Src and Erk. Imatinib ablated Crk pY221 phosphorylation in MDA-MB-231, indicating that Abl/Arg-based signaling was inhibited. Crk pY221 phosphorylation was partially impaired in imatinib-treated HNSCC lines (Figure 5a) and was further downregulated 
when combined with the Src inhibitor SU6656 (59) (Supplementary Figure 5b), suggesting that Crk is targeted by Src and Abl kinases in HNSCC.

Since Src and Erk regulate invadopodia in part by phosphorylating cortactin $(28,36,40)$, cortactin tyrosine and serine phosphorylation was evaluated in imatinib-treated HNSCC and MDA-MB-231 cells. The Erk-targeted cortactin residues S405 and S418 demonstrated elevated phosphorylation in imatinib-treated OSC19 and UMSCC1 cells, corresponding with increased Erk 1/2 activation, while treated MDA-MB-231 cells did not demonstrate a substantial increase in cortactin phosphorylation (Figure 5b). Imatinib stimulated cortactin Y421 phosphorylation in HNSCC cells (Figure 5c), likely due to increased Src activation (Figure 5a). These data support activation of the EGFRSrc/Erk-cortactin pathway by imatinib in HNSCC cells that can bypass Abl/Arg inhibition to promote invadopodia activity and HNSCC invasion.

\section{Imatinib stimulates HB-EGF synthesis and promotes HNSCC invadopodia activity}

Imatinib treatment of multiple HNSCC lines results in synthesis and secretion of the EGFR ligand HB-EGF, enhancing EGFR activity (60). Lysates from imatinib treated OSC19 and UMSCC1 cells contained increased HB-EGF compared to controls, whereas HB-EGF levels in MDA-MB-231 cells were unaltered (Figure 6a). Conditioned media from imatinib-treated HNSCC cells contained increased soluble HB-EGF at levels 2.5-3.0 fold higher than from MDA-MB-231 cells (Figure 6b). Addition of recombinant HB-EGF to cells at concentrations equivalent to HB-EGF levels in imatinib-treated conditioned media enhanced ECM degradation activity by $86 \%$ in OSC19 and $30 \%$ in UMSCC1 cells, but did not increase invadopodia activity in MDA-MB-231 cells (Figure $6 c-d)$. These results suggest that increased imatinib-induced HB-EGF expression and shedding by HNSCC cells produces an autocrine loop that stimulates EGFR activation responsible for enhancing invadopodia-mediated ECM proteolysis.

\section{Invadopodia ECM degradation promoted by impaired Abl kinase activity requires activation of EGFR and Src}

To further confirm that Src regulates HNSCC invadopodia downstream of EGFR apart from Abl kinases, pharmacological agents targeting EGFR, Src, or simultaneous Abl/Src 
inhibition were evaluated for their impact on gelatin degradation in HNSCC. UMSCC1 cells treated with the EGFR inhibitor gefitinib at $5 \mu \mathrm{M}$ reduced EGFR activation by $60 \%$ (Supplementary Figure 6a) and matrix degradation by 50\% (Figure 7a). Similar results were obtained with SU6656, where $15 \mu \mathrm{M}$ treatment resulted in a $65 \%$ decrease of the Src-targeted Y410 in p130CAS (Supplementary Figure $6 \mathrm{~b}$ ) and a 50\% reduction in matrix degradation (Figure 7b). Treatment of UMSCC1 cells with the dual Abl kinase/Src inhibitor saracatinib, $(61,62)$ at $1 \mu \mathrm{M}$ inhibited EGFR activity by $55 \%$, Src activity by $93 \%$, and Abl/Arg activity by $97 \%$ (Supplementary Figure 6c). ECM proteolysis was impaired by $80 \%$ (Figure 7c). Furthermore, the enhanced ECM degradation activity in UMSCC1 cells with Abl knockdown (Figure 1b-d) was abrogated with $10 \mu \mathrm{M}$ SU6656 (Figure 7d; Supplementary Figure 6d). These data indicate that the enhanced matrix degradation activity promoted by targeted Abl kinase inhibition in HNSCC requires intact EGFR-Src signaling. Similar results were obtained with saracatinib in OSC19 and MDA-MB-231 cells (Supplementary Figure 7a-c). 


\section{Discussion}

The effects of Abl-based signaling in solid tumors are currently controversial. Several studies have determined that Abl family kinase activity directly contributes to enhancing tumor proliferation, invasion and metastasis in breast and melanoma cell lines $(47,48,51,53,55)$. Abl family kinases also positively modulate tumorgenesis in gastric tumors (50) and non-small cell lung carcinoma (49). On the other hand, recent reports indicate that suppression of Abl kinase activity by imatinib increases breast tumor growth (86), invasion (63) and inhibits epithelial-to-mesenchymal transition (64). Imatinib also enhances thyroid cancer cell invasion (65). While these tumor stimulating findings have been attributed to use of mutationally modified Abl constructs or nonphysiological levels of imatinib (53), our results comparing HNSCC lines with MDA-MB231 cells indicate that $\mathrm{Abl}$ kinase inhibition by RNAi or clinically relevant imatinib concentrations yields opposite outcomes on tumor cell invasive events, suggesting that Abl kinases function to suppress HNSCC invasion by reducing invadopodia matrix degradation activity driven through the EGFR-Src-cortactin pathway.

Src activation is central to invadopodia formation and maturation, where phosphorylation of numerous downstream cytoskeletal proteins is required for invadopodia biogenesis and ECM proteolytic activity $(24,25)$. Cortactin phosphorylation downstream of $\mathrm{Src}$ is involved in all stages of invadopodia formation $(28,36,37,41)$. Src, $\mathrm{Abl}$ and Arg directly phosphorylate cortactin $(52,66,67)$, indicating that these kinases can promote cortactin-based Arp2/3 nucleation activity indirectly via NCK1 and NWASp. Src can also phosphorylate and activate Abl and Arg downstream of EGFR and other growth factor receptors $(54,68)$ and recent work has shown that removal of Abl or Arg by RNAi prevents invadopodia formation in Src transformed fibroblasts, MDA-MB231 breast cancer and melanoma cell lines $(51,53,55)$. While these studies indicate that $\mathrm{Abl}$ and $\mathrm{Arg}$ are the key cortactin kinases responsible for cortactin tyrosine phosphorylation in invadopodia (Figure 8a), we show that EGF stimulation of Abl/Argnull (DKO) fibroblasts enhances cortactin tyrosine phosphorylation, indicating that Src and/or other EGFR-activated cortactin targeting kinases phosphorylate cortactin apart from Abl or Arg. Src-transformed Abl/Arg-null fibroblasts retain the ability to degrade 
ECM and contain tyrosine phosphorylated cortactin within invadopodia, demonstrating that Abl family kinases are not essential in this system for invadopodia function driven by cortactin tyrosine phosphorylation. Abl re-expression in DKO cells impaired Srcgenerated ECM degradation, confirming an inhibitory role for Abl in invadopodia maturation similar to that observed in HNSCC cells. These results indicate that elevated levels of Src activity commonly present in HNSCC lines (36) or due to ectopic expression of active Src forms can circumvent the requirement for Abl or Arg in regulating invadopodia activity through cortactin phosphorylation (Figure 8b).

Imatinib-stimulated HNSCC invadopodia activity and invasion is likely due to increased activation of EGFR and associated downstream signaling, since imatinib treatment enhances EGFR, Src and Erk activation in HNSCC lines while having minimal impact on EGFR signaling in MDA-MB-231 cells. Imatinib treatment of HNSCC cells results in increased cortactin phosphorylation at Src-targeted Y421 and Erk-targeted S405/S418, phosphorylation events present within invadopodia required for ECM matrix degradation activity $(40,69,70)$. As in other HNSCC lines $(60)$, we observed that imatinib treatment increases synthesis and release of the EGFR ligand HB-EGF from OSC19 and UMSCC1 cells at concentrations 10-fold higher than MDA-MB-231 cells. Application of HB-EGF at imatinib-treated conditioned media concentrations enhanced invadopodia activity in HNSCC but not in MDA-MB-231 cells. This suggests that HNSCC cells lacking Abl or treated with imatinib generate an autocrine loop, where increased HBEGF synthesis and shedding in turn binds and activates EGFR to stimulate Src and Erk activation, leading to elevated cortactin phosphorylation and enhanced invadopodia ECM degradation (Figure 8b). HB-EGF induction of HNSCC invadopodia activity likely occurs in part through stimulation and secretion of MMP9 $(71,72)$, which localizes with UMSCC1 invadopodia at sites of ECM degradation (73). While our data are congruent with these findings, it is possible that other EGFR ligands may also be upregulated by Abl kinase suppression. The increased level of EGFR overexpression in HNSCC cells would render this tumor type more responsive to soluble HB-EGF than cell types that contain lower EGFR levels (e.g., MDA-MB-231), which may be the underlying reason for the differential response to Abl knockdown, imatinib and HB-EGF in our analyzed cell lines. Whether such a scenario applies to other EGFR overexpressing cancers 
and/or tumor types that display pro-invasive behavior in response to Abl kinase suppression will be important to determine.

How Abl kinase inhibition promotes HB-EGF synthesis and shedding in HNSCC is unclear. In addition to cytoskeletal regulation, Abl is a nuclear kinase and work in Ablnull and imatinib-treated fibroblasts indicates that $A b l$ functions to suppress NF-кB (nuclear factor-кB) activity through stabilization of the NF-кB regulator HDAC1 (74). Elevated NF-KB activity results in increased HB-EGF expression and EGFR activation (75), providing a link between Abl activation and negative regulation of HB-EGF expression. High HB-EGF expression is linked to poor clinical prognosis in HNSCC (76) and low Abl expression in HNSCC correlates with late stage tumors with poor outcome (77), supporting a potential connection between Abl activity and HB-EGF levels in driving HNSCC progression.

Pathway analysis with inhibitors of invadopodia signaling components confirmed that EGFR and Src activation in HNSCC is central to driving invadopodia-based ECM degradation. Simultaneous inhibition of $\mathrm{Src}$ and $\mathrm{Abl}$ kinases decreased matrix degradation by UMSCC1 and OSC19 cells, reinforcing the point that the elevated invadopodia activity in HNSCC resultant from Abl kinase inhibition requires concurrent Src activation. While preclinical studies on HNSCC lines has shown that saracatinib and the related inhibitor dasatinib impair cell invasion and display potent anti-tumor effects $(9,17)$, phase II trials in HNSCC patients with either drug as a single agent yielded no benefit in spite of apparent Src inhibition $(78,79)$. In contrast, a phase II trial with imatinib administered to HNSCC and NSCLC patients was closed early due to lack of efficacy and antagonistic effects, with a patient subset displaying a worse clinical outcome in response to imatinib (57). While not directly evaluated, these results support a role for Src inhibition in counteracting the pro-invasive effects of Abl kinase that would result in increased patient tumor progression. Although some response has been achieved with imatinib in solid tumors with combination approaches $(78,80-82)$, our results further emphasize the importance of careful patient selection and exclusion criteria for using imatinib or other Abl kinase inhibitors in HNSCC and other solid tumors that display similar characteristics. 
We have determined that $\mathrm{Abl}$ kinases have a context-dependent role in regulating invadopodia function and tumor invasion. In HNSCC cells Abl serves to suppress invadopodia ECM degradation and tumor invasion by preventing HB-EGF synthesis and extracellular shedding, where it is capable of activating the EGFR/Src/cortactin signaling pathway to accelerate invadopodia-based ECM degradation and tumor cell invasion. This is in contrast to MDA-MB-231 cells, a cell line commonly used to analyze invadopodia and invasive signaling, where Abl kinase inhibition prevents invadopodia activity and impairs invasiveness. These results stress the need for further mechanistic insight into the signaling processes that regulate the pro- and anti-oncogenic roles of Abl in solid tumors in order to prevent detrimental affects of imatinib treatment in ongoing and future patient trials. 


\section{Material and Methods}

\section{Plasmid constructs}

The lentiviral vector pLL5.0 (83) was used for Abl knockdown by subcloning a shRNA targeting human Abl oligonucleotide, (5'GCTCCGGGTCTTAGGCTAT3'; (84)) with Hpal and Xhol sites. For Abl knockdown-rescue experiments, the resulting vector was modified to encode a 6X-HA epitope tag using BamHI and Sbfl sites. Human Abl cDNA was PCR amplified from pMSCV-puro Abl (85) and subcloned into EcoRI and BamHI digested pLL5.0 6X-HA. Src-GFP constructs (WT, 527F, and 295M) were used as described (36).

\section{Cell culture, lentiviral infection and transfection}

UMSCC1, OSC19 and 1483 cells were maintained as described (36). $\mathrm{Abl}^{-1 /} / \mathrm{Arg}^{-/}$, wildtype MEFs, NIH3T3 and HEK 293T/17 cells were cultured as before (86). MDA-MB-231 cells were cultured in alpha Minimum Essential Media (Mediatech, Manassas, VA) supplemented with $10 \%$ fetal bovine serum (FBS; Hyclone, Logan UT), and $1 \%$ penicillin-streptomycin.

UMSCC1 cells stably infected with pLL5.0 6X-HA, pLL5.0 6X-HA Abl shRNA or pLL5.0 co-expressing Abl shRNA and HA-tagged Abl were generated by puromycin selection following standard methods. 1483, $\mathrm{Abl}^{-/ /} / \mathrm{Arg}^{-/-}$or wild-type MEF cells transiently expressing CMV-Src or HA-Abl constructs were transfected with the Nucleofector I device (Amaxa Biosystems, Berlin, Germany).

\section{Western blotting, antibodies and immunoprecipitation}

Western blotting of cell lysates was conducted as described (73). Antibodies used were: anti-Src clone GD11 (1:1000; EMD Millipore, Billerica, MA), anti-pY418 Src (1:1000; Invitrogen, Carlsbad, CA), anti-p130CAS (1:1000; BD Biosciences, San Jose, CA), anti-pY410 p130CAS (1:1000; Cell Signaling Technology, Danvers, MA), anticortactin clone 4F11 $(1 \mu \mathrm{g} / \mathrm{ml},(86))$, anti-pY421 cortactin (1:500; BD Biosciences), antipS405 cortactin (1:2000; (70)), anti-pS418 cortactin (1:500; (70)), anti-ERK1/2 clone C14 (1:1000; Santa Cruz Biotechnology, Santa Cruz, CA), anti-pERK1/2 (1:1000; Santa 
Cruz), anti- $\beta$-actin (1:10,000; EMD), anti-HA (1:200; Covance, Berkley, CA), anti-Crk (1:1000; BD), anti-pY221 Crk (1:1000; Cell Signaling), anti-PDGFR (1:200; Cell Signaling), anti-EGFR (1:1000, BD), anti-pY1068 EGFR (1:1000; BD), anti-Arg (1:500; EMD), anti-HB-EGF (1:200; EMD), and anti-Abl clone 8E9 (1:500; BD). Blots were quantified as described (73).

Immunoprecipitation was conducted from cells lysed in RIPA buffer (69). Clarified lysates $(250 \mu \mathrm{g})$ were incubated with $5 \mu \mathrm{g}$ anti-cortactin antibody $4 \mathrm{~F} 11$ for $2 \mathrm{~h}$ at $4{ }^{\circ} \mathrm{C}$. Immune complexes were captured by incubation with $30 \mu \mathrm{L}$ Protein $A / G$ beads (ThermoFisher Scientific, Rockford, IL) for $1 \mathrm{~h}$, washed with RIPA and analyzed by Western blotting.

\section{Invadopodia matrix degradation assays and fluorescence microscopy}

Cells were plated on Oregon Green 488-conjugated gelatin (Invitrogen, Grand Island, NY) coated coverslips $(27,87)$. In cases of inhibitor treatment, cells were allowed to attach for $1 \mathrm{~h}$, then serum starved for $12 \mathrm{~h}$ in the presence of $10 \mu \mathrm{M}$ GM6001 (Sigma) and either imatinib mesylate (LGM Pharmaceuticals, Boca Raton, FL), saracatnib (AstraZeneca, Alderley Park, Cheshire, United Kingdom), gefitinib (AstraZeneca) or SU6656 (EMD). Serum-free media was replaced with complete media containing $10 \%$ FBS and kinase inhibitors for $12 \mathrm{~h}$. Cells were rinsed in PBS, fixed in fresh $4 \%$ paraformaldehyde and labeled as described (36). Primary antibodies used were anticortactin clone 4F11 (1:500), anti-cortactin EP1922Y (1:500; Novus Biologicals, Littleton, CO), anti-cort-pY421 (1:500), anti-Src GD11 (1:500), anti-Abl clone 8E9 (1:200), anti-GFP (1:500; Invitrogen), and anti-HA (1:200). Primary antibodies were visualized using Alexa Fluor 405 and 647 conjugated goat anti-rabbit or anti-mouse secondary antibodies (1:2000; Invitrogen). F-actin was visualized using rhodamineconjugated phalloidin (1:500; Invitrogen). Cells were mounted with ProLong Gold (Invitrogen) and images were acquired with a Zeiss LSM510 confocal microscope using AIM software (Carl Zeiss Microlmaging, Thornwood, NY). Gelatin degradation was quantified as described previously (87). In brief, $\geq 90$ transiently transfected and $\geq 300$ lentiviral infected or inhibitor-treated cells evaluated for each condition. For therapeutic treatments and RNAi stable cell lines, the area of degradation and cell area was 
determined by analyzing the intensity of degraded gelatin or F-actin respectively in an entire field of view utilizing ImageJ software. For transient transfections, the area of degradation and cell area was determined by Image $\mathrm{J}$ software on an individual cell basis. The number of invadopodia per cell $(n \geq 50)$ and number of cells degrading matrix $(n \geq 100)$ were determined or each independent experiment $(n=\geq 3)(36)$.

\section{3-D spheroid invasion assays}

Cells were labeled with Vybrant ${ }^{\circledR}$ Dil (Invitrogen). 96 well plates were coated with 100 $\mu \mathrm{L}$ of $1.5 \%$ noble agar (BD Biosciences, Sparks, MD) in Dulbecco's PBS. $1 \times 10^{3}$ (OSC19), $5 \times 10^{3}$ (UMSCC1), or $2.5 \times 10^{3}$ (MDA-MB-231) labeled cells were plated into individual wells for $48 \mathrm{~h}$ to form spheroids. Two spheroids were transferred to a microcentrifuge tube and centrifuged at $1000 \times g$ for $3 \mathrm{~min}$. The media was aspirated and replaced with $500 \mu \mathrm{L}$ of $2 \mathrm{mg} / \mathrm{mL}$ rat tail collagen I (BD). The spheroid mixture was transferred to an individual well of 24 -well plate pre-coated with $400 \mu \mathrm{L}$ solidified 2 $\mathrm{mg} / \mathrm{mL}$ collagen I. Plates were incubated for $1 \mathrm{~h}$ at $37^{\circ} \mathrm{C}$ then overlayed with $1 \mathrm{~mL}$ of complete media. Spheroid invasion was visualized by fluorescence microscopy (Zeiss, Axiovert 200M) to establish the central z-axis $(0 \mathrm{~h})$ and imaged at 0 and $24 \mathrm{~h}$ by phase contrast microscopy. Spheroids were pretreated for $24 \mathrm{~h}$ and maintained in media with DMSO vehicle or $10 \mu \mathrm{M}$ imatinib. Maximal radial distances for invaded cells was calculated using Axiovision 4.6 software (Zeiss).

\section{HB-EGF ELISA assays}

HB-EGF specific enzyme-linked immunosorbent assay (ELISA) was performed according to the manufacturer's protocol (Abcam, Cambridge, MA). Cells were treated with imatinib $(10 \mu \mathrm{M})$ or DMSO for $12 \mathrm{~h}$, washed with PBS and incubated for $24 \mathrm{~h}$ in serum-free media with imatinib or DMSO. Conditioned media was concentrated to 500 $\mu \mathrm{L}$, and $100 \mu \mathrm{L}$ of media incubated overnight at $4^{\circ} \mathrm{C}$ in HB-EGF antibody-coated microplate strips. Absorbance values were obtained at $450 \mathrm{~nm}$ with a Biotek Synergy H1 Hybrid Reader (Winooski, VT). Standard curves were generated and results normalized to total cellular protein concentration for comparison across different cell lines. 


\section{Statistical analysis}

Differences in mean values between groups were evaluated using Students $t$-test and significance was determined at $P<0.05$. Scale bars represent confidence intervals (C.I.). 


\section{Conflict of Interest}

The authors declare they have no competing financial interests in relation to the work described. 


\section{Acknowledgements}

We thank Bruce Mayer (University of Connecticut) for Abl constructs, Anthony Koleske (Yale University) for $\mathrm{Abl}^{-1 /} / \mathrm{Arg}^{-1 /}$ fibroblasts, Silja Wessler (Paul-Ehrlich Institute) for the Abl shRNA construct, Elena Pugacheva (West Virginia University) for MDA-MB-231LN cells, Jim Bear (University of North Carolina) for pLL5.0 and advice on spheroid assay development. We thank Mark Auble and Barbara Frederick for technical assistance. Saracatinib and gefitinib were provided by AstraZeneca. Supported by NIH grants R01 DE014578, P20 RR16440 (to SAW) and the West Virginia University Mary Babb Randolph Cancer Center. The West Virginia University Microscopy Imaging Facility (supported by the Mary Babb Randolph Cancer, NIH grants P20 RR16440 and P30 RR032138/GM103488) is gratefully acknowledged. 


\section{$\underline{\text { References }}$}

(1) Pryor DI, Solomon B, Porceddu SV. The emerging era of personalized therapy in squamous cell carcinoma of the head and neck. Asia Pac J Clin Oncol 2011 Sep;7(3):236-251 .

(2) Leemans CR, Braakhuis BJ, Brakenhoff $\mathrm{RH}$. The molecular biology of head and neck cancer. Nat Rev Cancer 2011 Jan;11(1):9-22.

(3) Kalyankrishna S, Grandis JR. Epidermal growth factor receptor biology in head and neck cancer. J Clin Oncol 2006 Jun 10;24(17):2666-2672.

(4) Hama T, Yuza Y, Saito Y, O-uchi J, Kondo S, Okabe M, et al. Prognostic significance of epidermal growth factor receptor phosphorylation and mutation in head and neck squamous cell carcinoma. Oncologist 2009 Sep;14(9):900-908.

(5) Hama T, Yuza Y, Suda T, Saito Y, Norizoe C, Kato T, et al. Functional mutation analysis of EGFR family genes and corresponding lymph node metastases in head and neck squamous cell carcinoma. Clin Exp Metastasis 2012 Jan;29(1):19-25.

(6) Uribe P, Gonzalez S. Epidermal growth factor receptor (EGFR) and squamous cell carcinoma of the skin: Molecular bases for EGFR-targeted therapy. Pathology Research and Practice 2011 6/15;207(6):337-342.

(7) Fung C, Grandis JR. Emerging drugs to treat squamous cell carcinomas of the head and neck. Expert Opin Emerg Drugs 2010 Sep;15(3):355-373.

(8) Neiva KG, Zhang Z, Miyazawa M, Warner KA, Karl E, Nor JE. Cross talk initiated by endothelial cells enhances migration and inhibits anoikis of squamous cell carcinoma cells through STAT3/Akt/ERK signaling. Neoplasia 2009 Jun;11(6):583-593.

(9) Koppikar P, Choi SH, Egloff AM, Cai Q, Suzuki S, Freilino M, et al. Combined inhibition of c-Src and epidermal growth factor receptor abrogates growth and invasion of head and neck squamous cell carcinoma. Clin Cancer Res 2008 Jul 1;14(13):42844291.

(10) Yang JL, Qu XJ, Russell PJ, Goldstein D. Interferon-alpha promotes the antiproliferative effect of Erlotinib (OSI-774) on human colon cancer cell lines. Cancer Lett 2005 Jul 8;225(1):61-74. 
(11) Huang YT, Hwang JJ, Lee LT, Liebow C, Lee PP, Ke FC, et al. Inhibitory effects of a luteinizing hormone-releasing hormone agonist on basal and epidermal growth factorinduced cell proliferation and metastasis-associated properties in human epidermoid carcinoma A431 cells. Int J Cancer 2002 Jun 1;99(4):505-513.

(12) Guarino M. Src signaling in cancer invasion. J Cell Physiol 2010 Apr;223(1):14-26.

(13) Kim LC, Song L, Haura EB. Src kinases as therapeutic targets for cancer. Nat Rev Clin Oncol 2009 Oct;6(10):587-595.

(14) Summy JM, Gallick GE. Src family kinases in tumor progression and metastasis. Cancer Metastasis Rev 2003 Dec;22(4):337-358.

(15) Shor AC, Keschman EA, Lee FY, Muro-Cacho C, Letson GD, Trent JC, et al. Dasatinib inhibits migration and invasion in diverse human sarcoma cell lines and induces apoptosis in bone sarcoma cells dependent on SRC kinase for survival. Cancer Res 2007 Mar 15;67(6):2800-2808.

(16) Chen YS, Wu MJ, Huang CY, Lin SC, Chuang TH, Yu CC, et al. CD133/Src axis mediates tumor initiating property and epithelial-mesenchymal transition of head and neck cancer. PLoS One 2011;6(11):e28053.

(17) Johnson FM, Saigal B, Talpaz M, Donato NJ. Dasatinib (BMS-354825) tyrosine kinase inhibitor suppresses invasion and induces cell cycle arrest and apoptosis of head and neck squamous cell carcinoma and non-small cell lung cancer cells. Clin Cancer Res 2005 Oct 1;11(19 Pt 1):6924-6932.

(18) Sen B, Saigal B, Parikh N, Gallick G, Johnson FM. Sustained Src inhibition results in signal transducer and activator of transcription 3 (STAT3) activation and cancer cell survival via altered Janus-activated kinase-STAT3 binding. Cancer Res 2009 Mar 1;69(5):1958-1965.

(19) Zhang S, Yu D. Targeting Src family kinases in anti-cancer therapies: turning promise into triumph. Trends Pharmacol Sci 2012 3;33(3):122-128.

(20) Mayer EL, Krop IE. Advances in targeting SRC in the treatment of breast cancer and other solid malignancies. Clin Cancer Res 2010 Jul 15;16(14):3526-3532.

(21) Wheeler DL, lida M, Kruser TJ, Nechrebecki MM, Dunn EF, Armstrong EA, et al. Epidermal growth factor receptor cooperates with Src family kinases in acquired resistance to cetuximab. Cancer Biol Ther 2009 Apr;8(8):696-703. 
(22) Haura EB, Tanvetyanon T, Chiappori A, Williams C, Simon G, Antonia S, et al. Phase I/II study of the Src inhibitor dasatinib in combination with erlotinib in advanced non-small-cell lung cancer. J Clin Oncol 2010 Mar 10;28(8):1387-1394.

(23) Araujo JC, Mathew P, Armstrong AJ, Braud EL, Posadas E, Lonberg M, et al. Dasatinib combined with docetaxel for castration-resistant prostate cancer: results from a phase 1-2 study. Cancer 2012 Jan 1;118(1):63-71.

(24) Murphy DA, Courtneidge SA. The 'ins' and 'outs' of podosomes and invadopodia: characteristics, formation and function. Nat Rev Mol Cell Biol 2011 Jun 23;12(7):413426.

(25) Linder S, Wiesner C, Himmel M. Degrading devices: invadosomes in proteolytic cell invasion. Annu Rev Cell Dev Biol 2011 Nov 10;27:185-211.

(26) Poincloux R, Lizarraga F, Chavrier P. Matrix invasion by tumour cells: a focus on MT1-MMP trafficking to invadopodia. J Cell Sci 2009 Sep 1;122(Pt 17):3015-3024.

(27) Artym VV, Zhang Y, Seillier-Moiseiwitsch F, Yamada KM, Mueller SC. Dynamic interactions of cortactin and membrane type 1 matrix metalloproteinase at invadopodia: defining the stages of invadopodia formation and function. Cancer Res $2006 \mathrm{Mar}$ 15;66(6):3034-3043.

(28) Oser M, Yamaguchi H, Mader CC, Bravo-Cordero JJ, Arias M, Chen X, et al. Cortactin regulates cofilin and N-WASp activities to control the stages of invadopodium assembly and maturation. J Cell Biol 2009 Aug 24;186(4):571-587.

(29) Destaing O, Block MR, Planus E, Albiges-Rizo C. Invadosome regulation by adhesion signaling. Curr Opin Cell Biol 2011 Oct;23(5):597-606.

(30) Ren G, Crampton MS, Yap AS. Cortactin: Coordinating adhesion and the actin cytoskeleton at cellular protrusions. Cell Motil Cytoskeleton 2009 Oct;66(10):865-873.

(31) Buccione R, Caldieri G, Ayala I. Invadopodia: specialized tumor cell structures for the focal degradation of the extracellular matrix. Cancer Metastasis Rev 2009 Jun;28(12):137-149.

(32) Kirkbride KC, Sung BH, Sinha S, Weaver AM. Cortactin: a multifunctional regulator of cellular invasiveness. Cell Adh Migr 2011 Mar-Apr;5(2):187-198. 
(33) Weaver AM, Karginov AV, Kinley AW, Weed SA, Li Y, Parsons JT, et al. Cortactin promotes and stabilizes Arp2/3-induced actin filament network formation. Curr Biol 2001 Mar 6;11(5):370-374.

(34) Ammer AG, Weed SA. Cortactin branches out: roles in regulating protrusive actin dynamics. Cell Motil Cytoskeleton 2008 Sep;65(9):687-707.

(35) Martinez-Quiles N, Ho HY, Kirschner MW, Ramesh N, Geha RS. Erk/Src phosphorylation of cortactin acts as a switch on-switch off mechanism that controls its ability to activate N-WASP. Mol Cell Biol 2004 Jun;24(12):5269-5280.

(36) Kelley LC, Ammer AG, Hayes KE, Martin KH, Machida K, Jia L, et al. Oncogenic Src requires a wild-type counterpart to regulate invadopodia maturation. J Cell Sci 2010 Nov 15;123(Pt 22):3923-3932.

(37) Oser M, Mader CC, Gil-Henn H, Magalhaes M, Bravo-Cordero JJ, Koleske AJ, et al. Specific tyrosine phosphorylation sites on cortactin regulate Nck1-dependent actin polymerization in invadopodia. J Cell Sci 2010 Nov 1;123(Pt 21):3662-3673.

(38) Tehrani S, Tomasevic N, Weed S, Sakowicz R, Cooper JA. Src phosphorylation of cortactin enhances actin assembly. Proc Natl Acad Sci U S A 2007 Jul 17;104(29):11933-11938.

(39) Yamaguchi H, Lorenz M, Kempiak S, Sarmiento C, Coniglio S, Symons M, et al. Molecular mechanisms of invadopodium formation: the role of the N-WASP-Arp2/3 complex pathway and cofilin. J Cell Biol 2005 Jan 31;168(3):441-452.

(40) Ayala I, Baldassarre M, Giacchetti G, Caldieri G, Tete S, Luini A, et al. Multiple regulatory inputs converge on cortactin to control invadopodia biogenesis and extracellular matrix degradation. J Cell Sci 2008 Feb 1;121(Pt 3):369-378.

(41) Webb BA, Jia L, Eves R, Mak AS. Dissecting the functional domain requirements of cortactin in invadopodia formation. Eur J Cell Biol 2007 Apr;86(4):189-206.

(42) Colicelli J. ABL tyrosine kinases: evolution of function, regulation, and specificity. Sci Signal 2010 Sep 14;3(139):re6.

(43) Bradley WD, Koleske AJ. Regulation of cell migration and morphogenesis by Ablfamily kinases: emerging mechanisms and physiological contexts. J Cell Sci 2009 Oct 1;122(Pt 19):3441-3454. 
(44) Plattner R, Pendergast AM. Activation and signaling of the Abl tyrosine kinase: bidirectional link with phosphoinositide signaling. Cell Cycle 2003 Jul-Aug;2(4):273-274.

(45) Ernst T, Hochhaus A. Chronic myeloid leukemia: clinical impact of BCR-ABL1 mutations and other lesions associated with disease progression. Semin Oncol 2012 Feb;39(1):58-66.

(46) Wang Y, Gu M, Mi Y, Qiu L, Bian S, Wang J. Clinical characteristics and outcomes of mixed phenotype acute leukemia with Philadelphia chromosome positive and/or bcrabl positive in adult. Int J Hematol 2011 Dec;94(6):552-555.

(47) Srinivasan D, Plattner R. Activation of Abl tyrosine kinases promotes invasion of aggressive breast cancer cells. Cancer Res 2006 Jun 1;66(11):5648-5655.

(48) Srinivasan D, Sims JT, Plattner R. Aggressive breast cancer cells are dependent on activated $\mathrm{Abl}$ kinases for proliferation, anchorage-independent growth and survival. Oncogene 2008 Feb 14;27(8):1095-1105.

(49) Lin J, Sun T, Ji L, Deng W, Roth J, Minna J, et al. Oncogenic activation of c-Abl in non-small cell lung cancer cells lacking FUS1 expression: inhibition of c-Abl by the tumor suppressor gene product Fus1. Oncogene 2007 Oct 25;26(49):6989-6996.

(50) Furlan A, Stagni V, Hussain A, Richelme S, Conti F, Prodosmo A, et al. Abl interconnects oncogenic Met and p53 core pathways in cancer cells. Cell Death Differ 2011 Oct;18(10):1608-1616.

(51) Mader CC, Oser M, Magalhaes MA, Bravo-Cordero JJ, Condeelis J, Koleske AJ, et al. An EGFR-Src-Arg-cortactin pathway mediates functional maturation of invadopodia and breast cancer cell invasion. Cancer Res 2011 Mar 1;71(5):1730-1741.

(52) Lapetina S, Mader CC, Machida K, Mayer BJ, Koleske AJ. Arg interacts with cortactin to promote adhesion-dependent cell edge protrusion. J Cell Biol 2009 May 4;185(3):503-519.

(53) Ganguly SS, Fiore LS, Sims JT, Friend JW, Srinivasan D, Thacker MA, et al. c-Abl and Arg are activated in human primary melanomas, promote melanoma cell invasion via distinct pathways, and drive metastatic progression. Oncogene 2011 Sep 5.

(54) Plattner R, Kadlec L, DeMali KA, Kazlauskas A, Pendergast AM. c-Abl is activated by growth factors and $\mathrm{Src}$ family kinases and has a role in the cellular response to PDGF. Genes Dev 1999 Sep 15;13(18):2400-2411. 
(55) Smith-Pearson PS, Greuber EK, Yogalingam G, Pendergast AM. Abl kinases are required for invadopodia formation and chemokine-induced invasion. J Biol Chem 2010 Dec 17;285(51):40201-40211.

(56) Sibony-Benyamini H, Gil-Henn H. Invadopodia: The leading force. Eur J Cell Biol 2012 May 24.

(57) Tsao AS, Liu S, Fujimoto J, Wistuba II, Lee JJ, Marom EM, et al. Phase II trials of imatinib mesylate and docetaxel in patients with metastatic non-small cell lung cancer and head and neck squamous cell carcinoma. J Thorac Oncol 2011 Dec;6(12):21042111.

(58) Clark ES, Whigham AS, Yarbrough WG, Weaver AM. Cortactin is an essential regulator of matrix metalloproteinase secretion and extracellular matrix degradation in invadopodia. Cancer Res 2007 May 1;67(9):4227-4235.

(59) Blake RA, Broome MA, Liu X, Wu J, Gishizky M, Sun L, et al. SU6656, a selective src family kinase inhibitor, used to probe growth factor signaling. Mol Cell Biol 2000 Dec;20(23):9018-9027.

(60) Johnson FM, Saigal B, Donato NJ. Induction of heparin-binding EGF-like growth factor and activation of EGF receptor in imatinib mesylate-treated squamous carcinoma cells. J Cell Physiol 2005 Nov;205(2):218-227.

(61) Hennequin LF, Allen J, Breed J, Curwen J, Fennell M, Green TP, et al. N-(5-chloro1,3-benzodioxol-4-yl)-7-[2-(4-methylpiperazin-1-yl)ethoxy]-5- (tetrahydro-2H-pyran-4yloxy)quinazolin-4-amine, a novel, highly selective, orally available, dual-specific cSrc/Abl kinase inhibitor. J Med Chem 2006 Nov 2;49(22):6465-6488.

(62) Summy JM, Gallick GE. Treatment for advanced tumors: SRC reclaims center stage. Clin Cancer Res 2006 Mar 1;12(5):1398-1401.

(63) Noren NK, Foos G, Hauser CA, Pasquale EB. The EphB4 receptor suppresses breast cancer cell tumorigenicity through an Abl-Crk pathway. Nat Cell Biol 2006 Aug;8(8):815-825.

(64) Allington TM, Galliher-Beckley AJ, Schiemann WP. Activated Abl kinase inhibits oncogenic transforming growth factor-beta signaling and tumorigenesis in mammary tumors. FASEB J 2009 Dec;23(12):4231-4243. 
(65) Frasca F, Vigneri P, Vella V, Vigneri R, Wang JY. Tyrosine kinase inhibitor STI571 enhances thyroid cancer cell motile response to Hepatocyte Growth Factor. Oncogene 2001 Jun 28;20(29):3845-3856.

(66) Huang C, Liu J, Haudenschild CC, Zhan X. The role of tyrosine phosphorylation of cortactin in the locomotion of endothelial cells. J Biol Chem 1998 Oct 2;273(40):2577025776.

(67) Boyle SN, Michaud GA, Schweitzer B, Predki PF, Koleske AJ. A critical role for cortactin phosphorylation by Abl-family kinases in PDGF-induced dorsal-wave formation. Curr Biol 2007 Mar 6;17(5):445-451.

(68) Plattner R, Koleske AJ, Kazlauskas A, Pendergast AM. Bidirectional signaling links the Abelson kinases to the platelet-derived growth factor receptor. Mol Cell Biol 2004 Mar;24(6):2573-2583.

(69) Head JA, Jiang D, Li M, Zorn LJ, Schaefer EM, Parsons JT, et al. Cortactin tyrosine phosphorylation requires Rac1 activity and association with the cortical actin cytoskeleton. Mol Biol Cell 2003 Aug;14(8):3216-3229.

(70) Kelley LC, Hayes KE, Ammer AG, Martin KH, Weed SA. Cortactin phosphorylated by ERK1/2 localizes to sites of dynamic actin regulation and is required for carcinoma lamellipodia persistence. PLoS One 2010 Nov 4;5(11):e13847.

(71) O-charoenrat P, Modjtahedi H, Rhys-Evans P, Court WJ, Box GM, Eccles SA. Epidermal growth factor-like ligands differentially up-regulate matrix metalloproteinase 9 in head and neck squamous carcinoma cells. Cancer Res 2000 Feb 15;60(4):11211128.

(72) Ohnishi Y, Inoue H, Furukawa M, Kakudo K, Nozaki M. Heparin-binding epidermal growth factor-like growth factor is a potent regulator of invasion activity in oral squamous cell carcinoma. Oncol Rep 2012 Apr;27(4):954-958.

(73) Ammer AG, Kelley LC, Hayes KE, Evans JV, Lopez-Skinner LA, Martin KH, et al. Saracatinib Impairs Head and Neck Squamous Cell Carcinoma Invasion by Disrupting Invadopodia Function. J Cancer Sci Ther 2009 Nov 30;1(2):52-61.

(74) Liberatore RA, Goff SP, Nunes I. NF-kappaB activity is constitutively elevated in cAbl null fibroblasts. Proc Natl Acad Sci U S A 2009 Oct 20;106(42):17823-17828. 
(75) Wang F, Liu R, Lee SW, Sloss CM, Couget J, Cusack JC. Heparin-binding EGFlike growth factor is an early response gene to chemotherapy and contributes to chemotherapy resistance. Oncogene 2007 Mar 29;26(14):2006-2016.

(76) Hatakeyama $\mathrm{H}$, Cheng $\mathrm{H}$, Wirth $\mathrm{P}$, Counsell A, Marcrom SR, Wood CB, et al. Regulation of heparin-binding EGF-like growth factor by miR-212 and acquired cetuximab-resistance in head and neck squamous cell carcinoma. PLoS One $2010 \mathrm{Sep}$ 13;5(9):e12702.

(77) Yanagawa T, Harada H, Iwasa S, Tabuchi K, Omura K, Suzuki H, et al. c-Abl expression in oral squamous cell carcinomas. Oral Oncol 2000 Jan;36(1):89-94.

(78) Fury MG, Baxi S, Shen R, Kelly KW, Lipson BL, Carlson D, et al. Phase II study of saracatinib (AZD0530) for patients with recurrent or metastatic head and neck squamous cell carcinoma (HNSCC). Anticancer Res 2011 Jan;31(1):249-253.

(79) Brooks HD, Glisson BS, Bekele BN, Johnson FM, Ginsberg LE, El-Naggar A, et al. Phase 2 study of dasatinib in the treatment of head and neck squamous cell carcinoma. Cancer 2011 May 15;117(10):2112-2119.

(80) Montero JC, Seoane S, Ocana A, Pandiella A. Inhibition of SRC family kinases and receptor tyrosine kinases by dasatinib: possible combinations in solid tumors. Clin Cancer Res 2011 Sep 1;17(17):5546-5552.

(81) Lara PN,Jr, Longmate J, Evans CP, Quinn DI, Twardowski P, Chatta G, et al. A phase II trial of the Src-kinase inhibitor AZD0530 in patients with advanced castrationresistant prostate cancer: a California Cancer Consortium study. Anticancer Drugs 2009 Mar;20(3):179-184.

(82) Gucalp A, Sparano JA, Caravelli J, Santamauro J, Patil S, Abbruzzi A, et al. Phase II trial of saracatinib (AZD0530), an oral SRC-inhibitor for the treatment of patients with hormone receptor-negative metastatic breast cancer. Clin Breast Cancer 2011 Oct;11(5):306-311.

(83) Cai L, Makhov AM, Schafer DA, Bear JE. Coronin 1B antagonizes cortactin and remodels Arp2/3-containing actin branches in lamellipodia. Cell 2008 Sep 5;134(5):828842.

(84) Poppe M, Feller SM, Romer G, Wessler S. Phosphorylation of Helicobacter pylori CagA by c-Abl leads to cell motility. Oncogene 2007 May 24;26(24):3462-3472. 
(85) Antoku S, Saksela K, Rivera GM, Mayer BJ. A crucial role in cell spreading for the interaction of Abl PxxP motifs with Crk and Nck adaptors. J Cell Sci 2008 Sep 15;121(Pt 18):3071-3082.

(86) Rothschild BL, Shim AH, Ammer AG, Kelley LC, Irby KB, Head JA, et al. Cortactin overexpression regulates actin-related protein $2 / 3$ complex activity, motility, and invasion in carcinomas with chromosome 11q13 amplification. Cancer Res 2006 Aug 15;66(16):8017-8025.

(87) Martin KH, Hayes KE, Walk EL, Ammer AG, Markwell SM, Weed SA. Quantitative Measurement of Invadopodia-mediated Extracellular Matrix Proteolysis in Single and Multicellular Contexts. J Vis Exp 2012 Aug 27;(66). pii: 4119. doi(66):10.3791/4119. 


\section{Figure Legends}

Figure 1 Abl expression inhibits invadopodia activity in HNSCC cells. (a) UMSCC1 cells transfected with empty vector (HA-Ctl) or HA-tagged Abl (HA-Abl) were plated on Oregon Green 488-gelatin coated coverslips (pseudocolored white) and incubated for $24 \mathrm{~h}$. Cells were fixed and labeled with anti-HA (blue), anti-cortactin (green) and rhodamine-phalloidin (F-Actin; red). Boxed areas and corresponding insets denote regions of HA-Abl localization with invadopodia markers and regions of matrix degradation. (b) Western blot analysis of Abl expression. Cell lysates $(100 \mu \mathrm{g})$ from UMSCC1 control (Ctl), shRNA Abl knockdown (shRNA) and wild-type (WT) Abl rescued shRNA cells. Parallel blots were probed for $\beta$-actin to confirm equal protein loading across all lines. Numbers denote different independent clones. (c) Representative confocal images of gelatin matrix degradation assays conducted with the indicated UMSCC1 clonal cell lines described in (b). Cells were plated on Oregon Green 488gelatin coated coverslips (pseudocolored white) for $9 \mathrm{~h}$, fixed and labeled with an anticortactin antibody (green) and rhodamine-phalloidin (F-Actin; red). The amount of gelatin degradation per cell area from four independent experiments was quantified in (d). Data are represented as mean \pm C.I.; ${ }^{* \star} P \leq 0.01$. Scale bars: $10 \mu \mathrm{m}$ in (a), $30 \mu \mathrm{m}$ in (c).

Figure 2 Targeted inhibition of Abl family kinases with imatinib has divergent effects on invadopodia activity in HNSCC and breast cancer cells. (a) OSC19, UMSCC1 (HNSCC) and MDA-MB-231 (breast cancer) cells were treated with the indicated concentrations of imatinib (STI571) for $24 \mathrm{~h}$, lysed and $25 \mu \mathrm{g}$ of cell lysate analyzed by Western blotting with anti-pY221 Crk to determine drug efficacy. The ratio of pY221 Crk phosphorylation to total Crk levels determined by densitometry is displayed between blots. Blots are representative from three to four independent experiments for each cell line. (b) OSC19, (c) UMSCC1, and (d) MDA-MB-231 cells were plated on Oregon Green 488-gelatin coated coverslips (pseudocolored white) in complete media for $1 \mathrm{~h}$ to

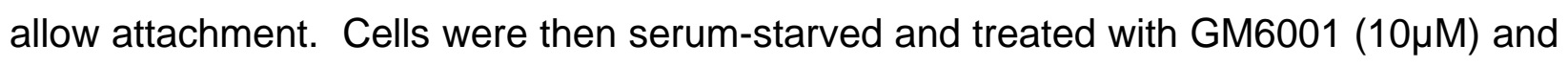
either imatinib (STI571) or DMSO vehicle $(0 \mu \mathrm{M})$ concomitantly for $12 \mathrm{~h}$. After GM6001 washout, cells were incubated with complete media containing $10 \%$ FBS for $12 \mathrm{~h}$ in the 
presence or absence of imatinib. Cells were fixed and labeled with an anti-cortactin antibody (green) and rhodamine-phalloidin (F-Actin; red) and visualized using confocal microscopy. Representative images showing cortactin/F-Actin labeling and gelatin degradation for each line and indicated experimental condition are shown. Insets denote areas of invadopodia activity indicated by the presence of overlapping cortactin/F-Actin puncta that co-localize with dark regions of Oregon Green 488-gelatin clearing. Scale bars: $20 \mu \mathrm{m}$. (e) Quantification of Oregon Green 488-gelatin degradation for OSC19, UMSCC1 and MDA-MB-231 cells treated with the indicated imatinib (STI571) concentrations. Data from 3 independent experiments for each experimental condition are represented as mean \pm C.I.; ${ }^{* \star} P \leq 0.01$.

Figure 3 Imatinib enhances HNSCC invasion through 3D collagen matrices. (a) OSC19, (b) UMSCC1 and (c) MDA-MB-231 tumor cell spheroids pre-treated with $10 \mu \mathrm{M}$ imatinib (STI571) or DMSO vehicle $(0 \mu \mathrm{M})$ for $24 \mathrm{~h}$ were embedded in collagen I $(0 \mathrm{~h})$. Spheroids were further incubated in complete media without $(0 \mu \mathrm{M})$ or with imatinib for an additional $24 \mathrm{~h}$ and invasion monitored by phase contrast microscopy. White circles indicate the maximum radial distance traveled by invaded cells. Scale bars: $200 \mu \mathrm{m}$. (d) Quantification of average invasive distance traveled by cells in each experimental condition ( $n \geq 12$ spheroids assayed per cell line and treatment). Data are represented as mean \pm C.I.; ${ }^{*} P \leq 0.01 ;{ }^{*} P \leq 0.05$.

Figure 4 Elevated Src activity bypasses Abl family kinase regulation of invadopodia function. (a) UMSCC1, OSC19 and MDA-MB-231 cells grown in complete media were analyzed by Western blotting of total cell lysates $(25-50 \mu \mathrm{g})$ for relative basal activation of EGFR, Src and Abl family kinases with the indicated phosphorylation-specific antibodies. Lysates were immunoblotted in parallel for total EGFR, Src, Crk, Abl and Arg protein levels. (b) $\mathrm{Abl}^{-/ /} / \mathrm{Arg}^{-/-}$double knockout (DKO) and wild-type (WT) mouse embryo fibroblasts (MEFs) were serum starved overnight, then stimulated with 100 $\mathrm{ng} / \mathrm{mL}$ EGF for $15 \mathrm{~min}$. Control and stimulated cells were lysed, and $50 \mu \mathrm{g}$ of total protein assayed by immunoblotting with phosphorylation-specific antibodies against cortactin pY421 (Cort pY421) and total cortactin. The ratio of pY421 cortactin relative to total cortactin levels for each cell type and treatment are shown. (c) WT and DKO cells 
transiently expressing constitutive active Src (527F) were plated on Oregon Green 488gelatin coated coverslips for $12 \mathrm{~h}$, fixed, and immunolabeled with anti-cortactin-pY421 (blue), anti-cortactin (green) and rhodamine-phalloidin (F-Actin; red). Cells and matrix were imaged by confocal microscopy. Insets show co-localization of pY421 cortactin within invadopodia at areas corresponding with clearing of Oregon Green 488-gelatin (pseudocolored white). DKO cells expressing GFP-tagged wild-type (WT), 527F, kinase inactive (K295M) Src, co-expressing 527F Src and wild-type (WT) Abl or wild-type (WT) MEFs expressing 527F Src were assayed for the percentage of cells degrading the ECM (d) and for normalized matrix degradation per cell area (e). Data are represented as mean \pm C.I. from 3 independent experiments. ${ }^{* *} P \leq 0.01$.

Figure 5 Imatinib activates the invadopodia kinase pathway in HNSCC cells. (a) OSC19, UMSCC1 and MDA-MB-231 cells grown in complete media were treated with DMSO vehicle $(0 \mu \mathrm{M})$ or the indicated imatinib (STI571) concentrations for $24 \mathrm{~h}$. Cells were lysed and $25-50 \mu \mathrm{g}$ of total cell protein were assayed by immunoblotting for activation of EGFR ( $p$ Y1068), Src (pY418), Erk (pErk) and Abl/Arg kinases (pY221 Crk). Parallel blots were probed for corresponding levels of each assayed protein. Ratios of phosphorylated/total protein are shown (represented as mean from $\geq 3$ independent experiments). All blots are representative images. (b) Western blot analysis of cortactin S405 and S418 phosphorylation from cells treated and prepared in (a) with the indicated phosphorylation-specific antibodies. A representative blot was stripped and reprobed with anti-cortactin to verify equal loading. (c) OSC19, UMSCC1 and MDAMB-231 cells treated as in (a) were lysed and cortactin was immunoprecipitated from $250 \mu \mathrm{g}$ of cell extract. Immune complexes were assayed by Western blotting for cortactin Y421 phosphorylation (anti-Cort-pY421). Parallel blots with anti-pan-cortactin were conducted to verify cortactin immunoprecipitation.

Figure 6 Imatinib induced HB-EGF stimulates HNSCC invadopodia ECM degradation. (a) OSC19, UMSCC1 and MDA-MB-231 cells treated with DMSO $(0 \mu \mathrm{M})$ or imatinib mesylate $(\mathrm{STI571}, 10 \mu \mathrm{M})$ for $24 \mathrm{~h}$ were lysed and $100 \mu \mathrm{g}$ cell protein assayed by Western blot analysis for HB-EGF. Bracketed HB-EGF shows different HB-EGF posttranslationally modified forms. Positive control (+ Ctl) recombinant HB-EGF ectodomain 
is denoted by an asterisk $\left({ }^{*}\right)$. A parallel blot was probed with anti- $\beta$-actin to confirm equal protein loading. (b) HB-EGF ELISA analysis of conditioned media from cells treated with the indicated imatinib (STI571) concentrations. Concentrations of cleaved, soluble HB-EGF were adjusted in accordance with total cellular protein levels after treatment from each cell line to allow cross-comparison. (c) Soluble HB-EGF promotes HNSCC invadopodia ECM degradation. OSC19, UMSCC1, and MDA-MB-231 cells were plated on Oregon Green 488-gelatin coated coverslips in complete media for $2 \mathrm{~h}$ to allow attachment and stimulated with recombinant $\operatorname{HB}-E G F(0,25$, or $250 \mathrm{pg} / \mathrm{mL})$ for 7 h. HB-EGF concentrations were calculated from average levels present in conditioned media of imatinib-treated cells. Cells were fixed and labeled with an anticortactin antibody (green) and rhodamine-phalloidin (F-Actin; red) and visualized using confocal microscopy. Insets denote areas of invadopodia activity. Scale bars: $20 \mu \mathrm{m}$. (d) Quantification of Oregon Green 488-gelatin degradation from OSC19, UMSCC1 and MDA-MB-231 cells treated with the indicated HB-EGF concentrations as in (c). Data are represented as mean \pm C.I.; ${ }^{*} P \leq 0.01 ;{ }^{*} P \leq 0.05$.

Figure 7 Targeted inhibition of the EGFR-Src pathway impairs HNSCC invadopodia activity. UMSCC1 cells treated with the indicated concentrations of gefitinib (a), SU6656 (b) and saracatinib (c) for $24 \mathrm{~h}$ were fixed and evaluated for Oregon Green 488-gelatin degradation by fluorescence microscopy with rhodamine-phalloidin and anticortactin antibodies. (d) UMSCC1 control (Ctl 8), shRNA Abl knockdown (shRNA 3) and Abl-rescued shRNA cells (WT 6) were treated with vehicle $(0 \mu \mathrm{M})$ or SU6656 (10 $\mu \mathrm{M})$ and assayed for effects on Oregon Green 488-gelatin proteolysis as above. Cells and matrix were imaged by confocal microscopy and the level of matrix degradation quantified for each treatment condition. Data are represented as mean \pm C.I.; ** $P \leq 0.01 ;{ }^{*} P \leq 0.05$.

Figure 8 Schematic diagram of EGFR-invadopodia signaling pathways altered by Abl inhibition in HNSCC. (a) The current invadopodia pathway depicting EGFR activation to cortactin phosphorylation as primarily determined in breast cancer cell lines. EGFR activation increases Src and Erk1/2 kinase activity, where Src stimulates Abl/Arg activation resulting in direct Abl/Arg cortactin tyrosine phosphorylation, while Erk1/2 
directly phosphorylates cortactin S405/418. (b) In HNSCC, inhibition of Abl/Arg results in increased activation of EGFR through elevated HB-EGF synthesis and shedding, resulting in autocrine EGFR hyperstimulation. This leads to elevated Src activity that bypasses Abl/Arg and directly phosphorylates cortactin. Erk1/2 activation is also increased under these conditions, where the combined effect of enhanced Src and Erk1/2 activation increases cortactin phosphorylation and correlates with elevated HNSCC-mediated ECM degradation and invasion. 
A

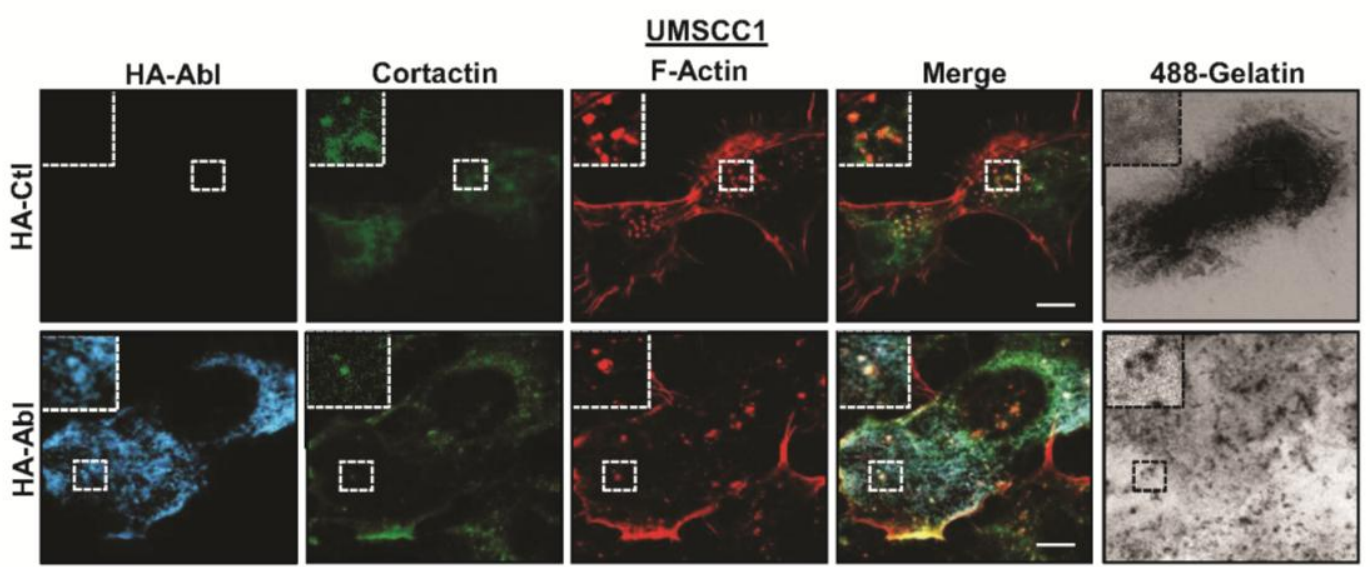

B

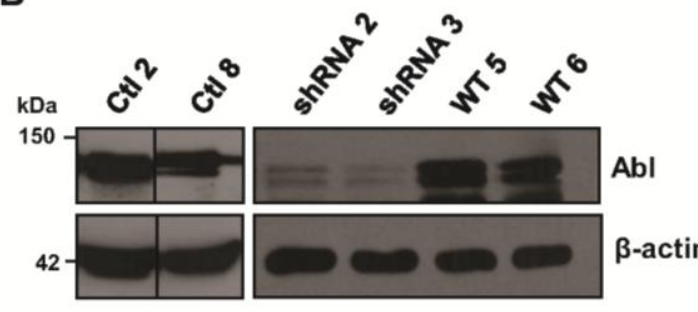

D

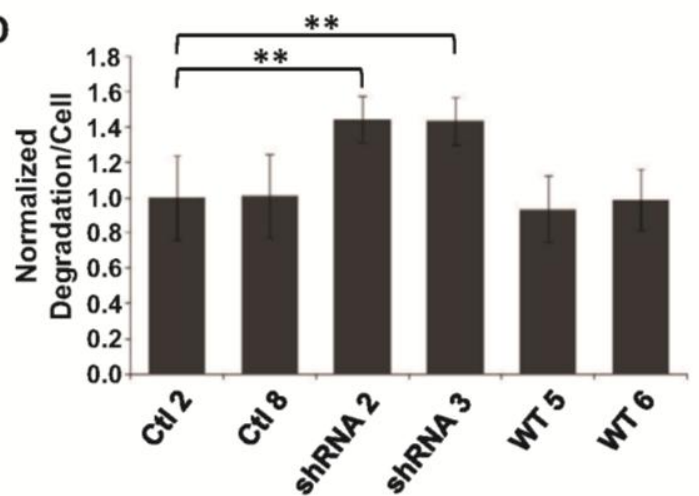

C

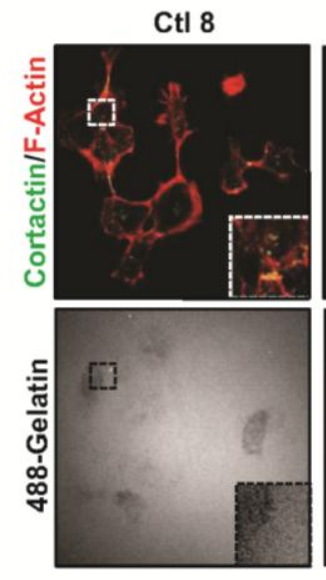

ShRNA 2

WT 5
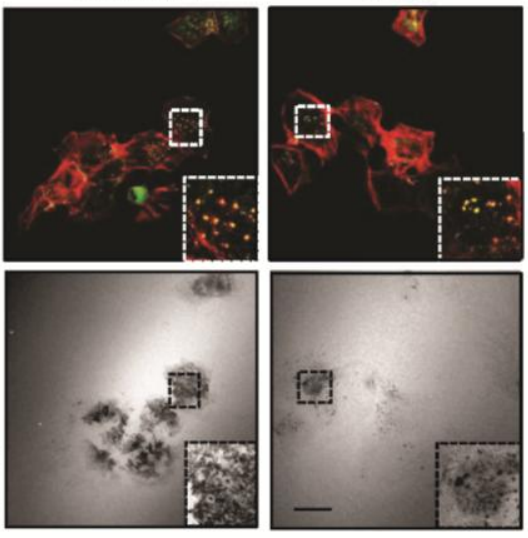

Hayes et al. Figure 1 
A

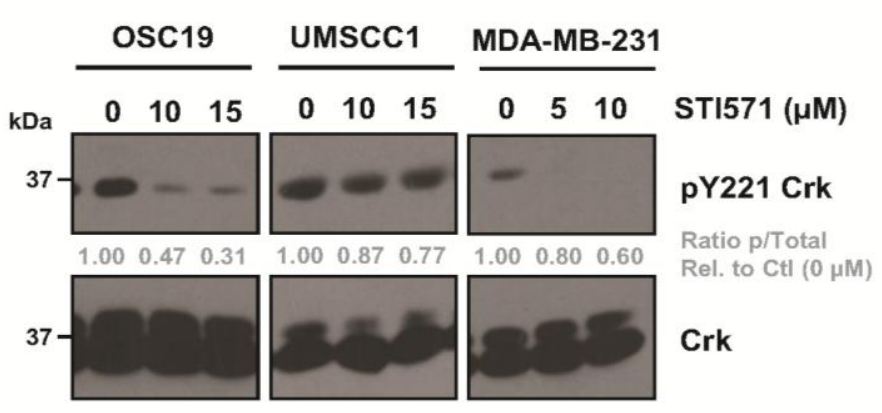

C

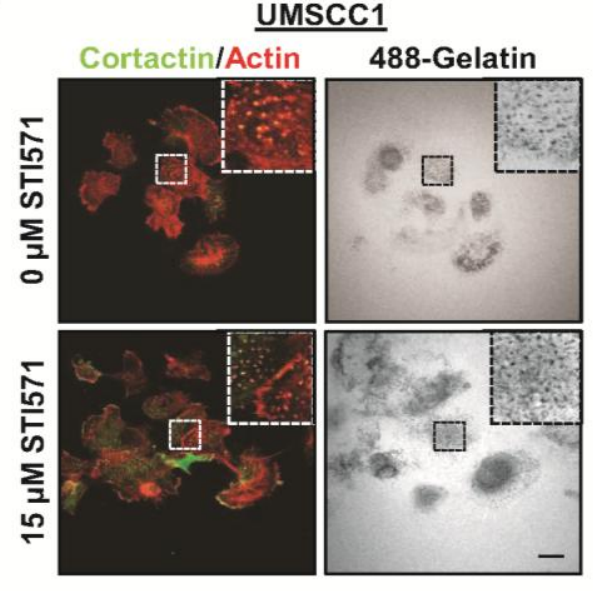

B

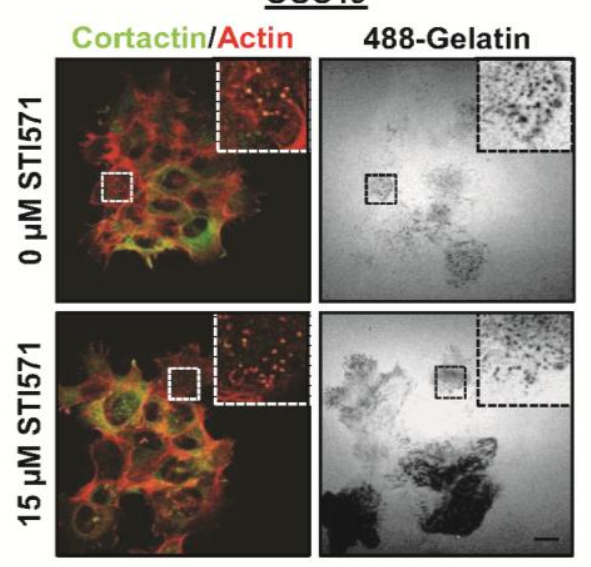

D

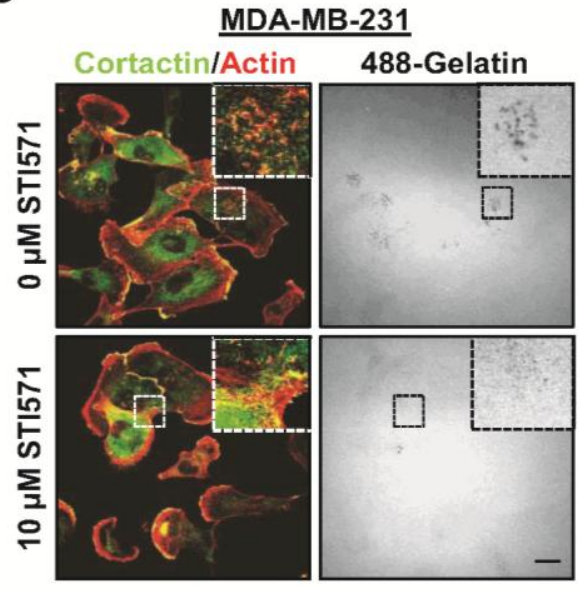

E

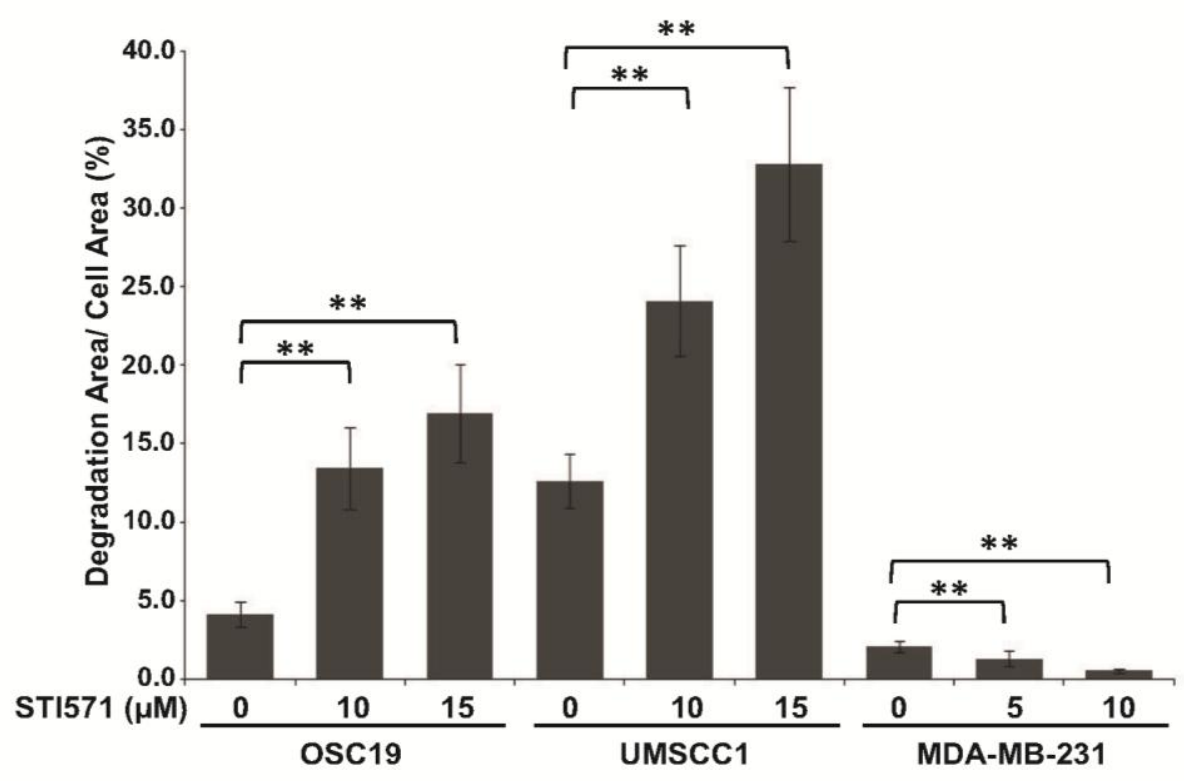

Hayes et al. Figure 2 
A

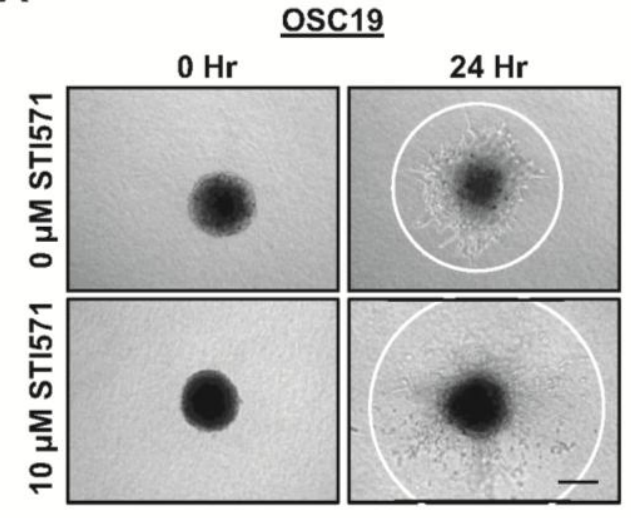

C

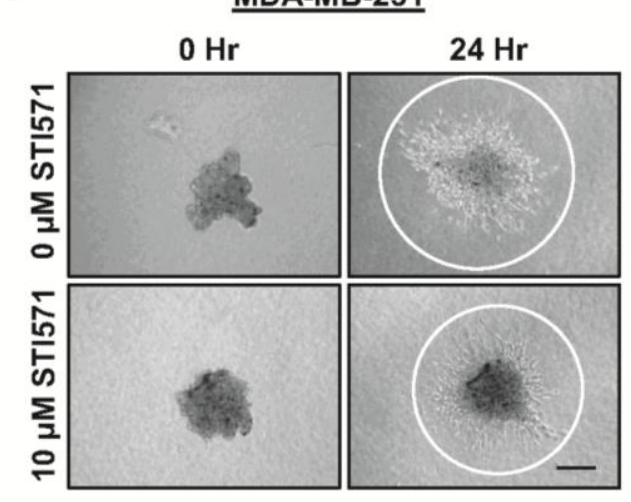

B

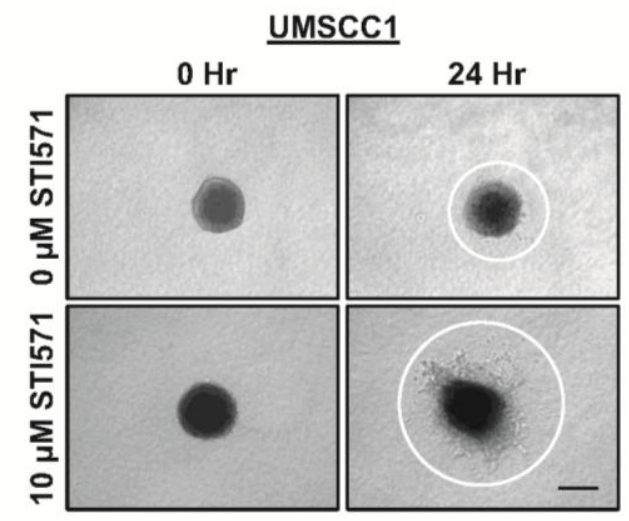

D

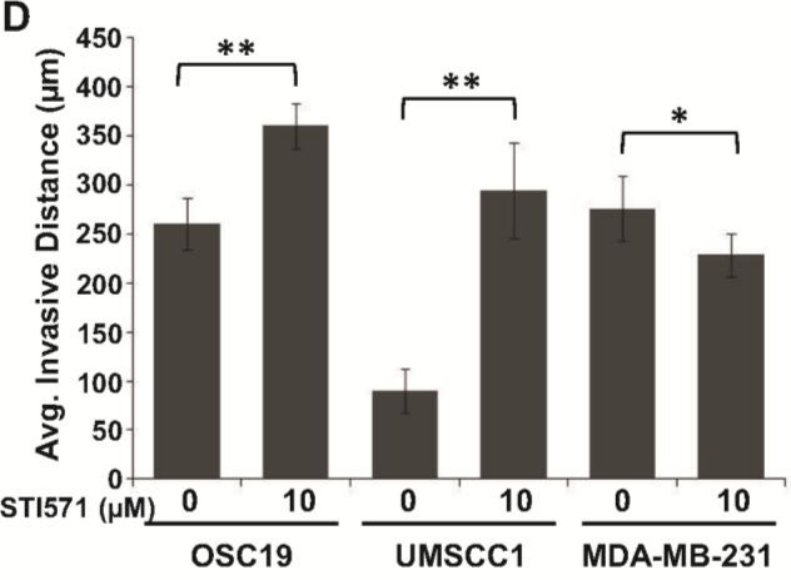

Hayes et al. Figure 3 
A

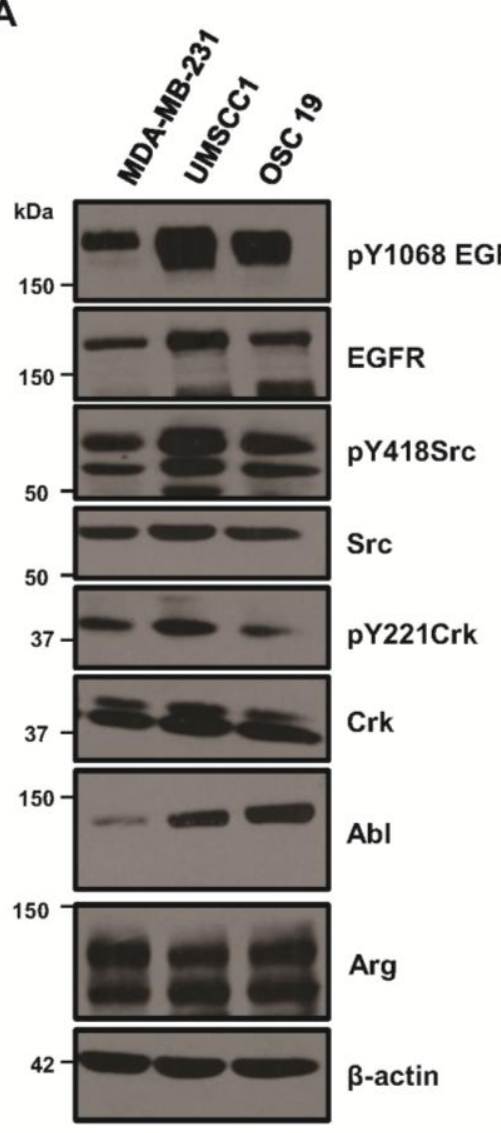

D

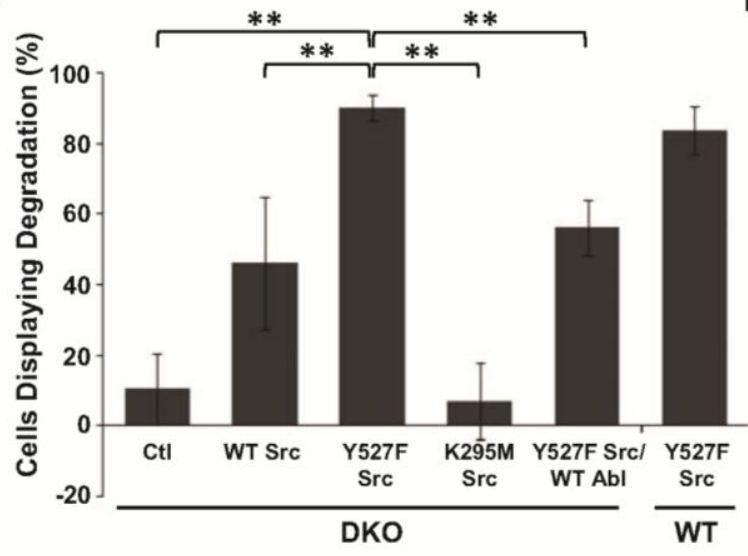

C
B

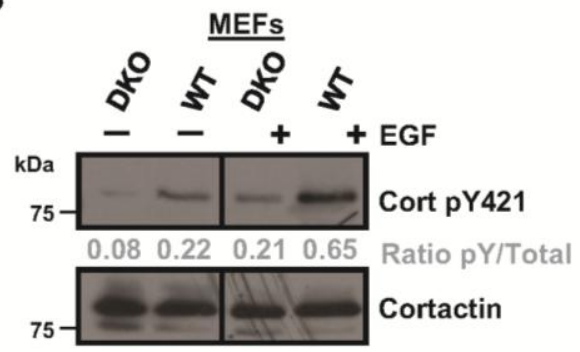

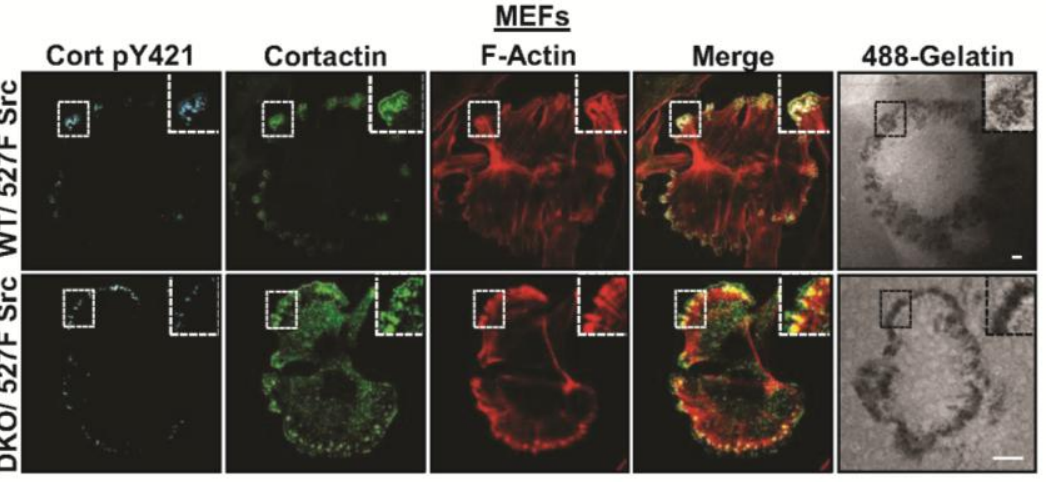

E

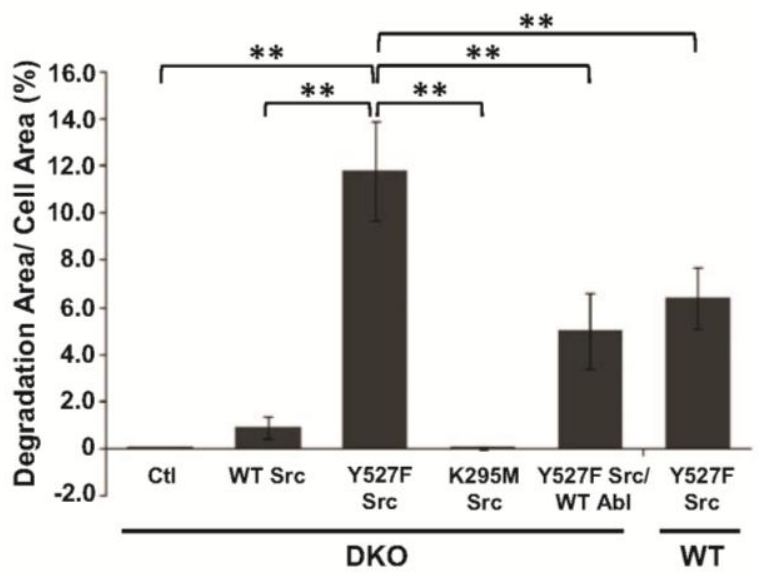

Hayes et al. Figure 4 
A
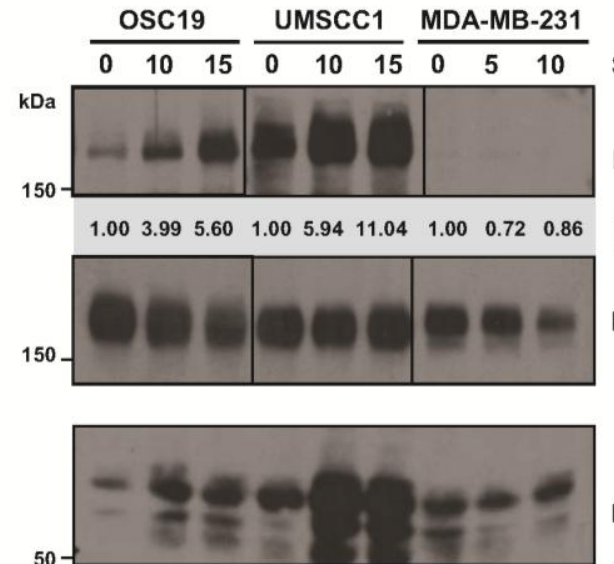

$\begin{array}{llllllllll}1.00 & 1.58 & 3.13 & 1.00 & 1.73 & 2.00 & 1.00 & 1.02 & 1.71\end{array}$

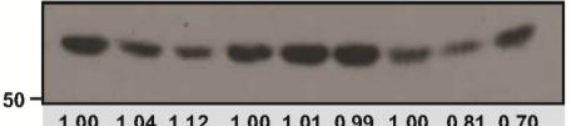

$\begin{array}{lllllllll}1.00 & 1.04 & 1.12 & 1.00 & 1.01 & 0.99 & 1.00 & 0.81 & 0.70\end{array}$

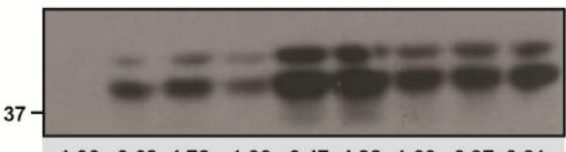

$\begin{array}{lllllllll}1.00 & 3.68 & 4.78 & 1.00 & 6.47 & 4.82 & 1.00 & 0.97 & 0.91\end{array}$
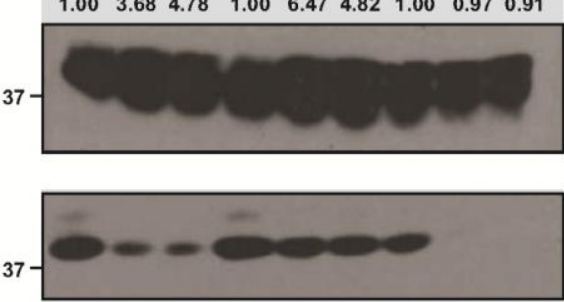

$\begin{array}{lllllllll}1.00 & 0.38 & 0.34 & 1.00 & 0.64 & 0.67 & 1.00 & 0.32 & 0.17\end{array}$
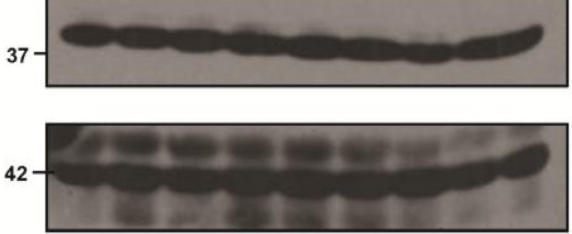

Erk

\section{pY221 Crk}

Ratio $\mathrm{p} /$ Total

Rel. to Ctl $(0 \mu \mathrm{M})$

Crk

$\beta$-actin
pErk

Ratio $p /$ Total Rel, to Ctl $(0 \mu \mathrm{M})$

B

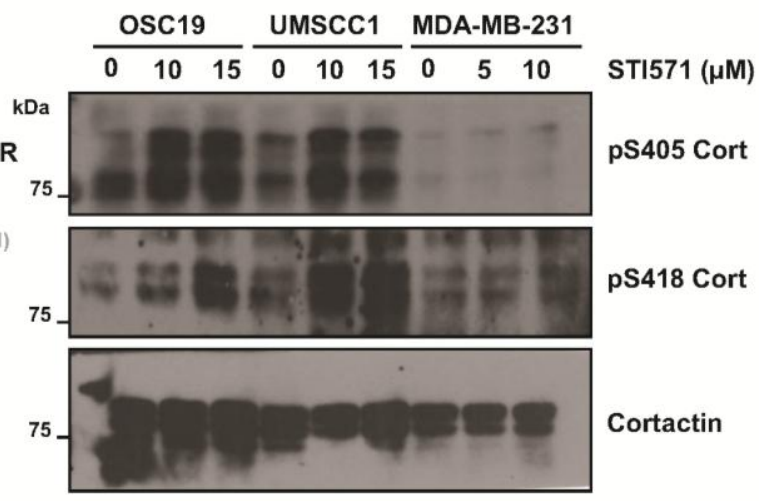

pY418 Src

Src C

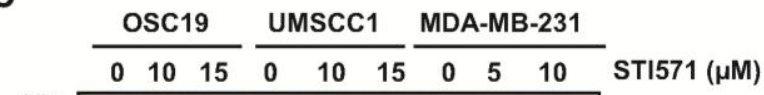

$\mathrm{kDa}$

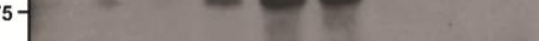

IP: Cortactin

IB: pY421 Cort

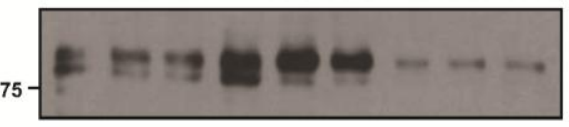

IP: Cortactin

IB: Cortactin

Hayes et al. Figure 5 
A

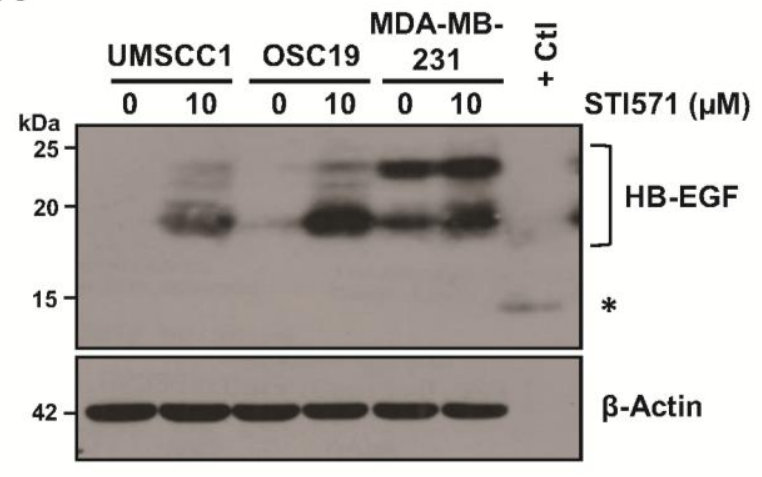

B

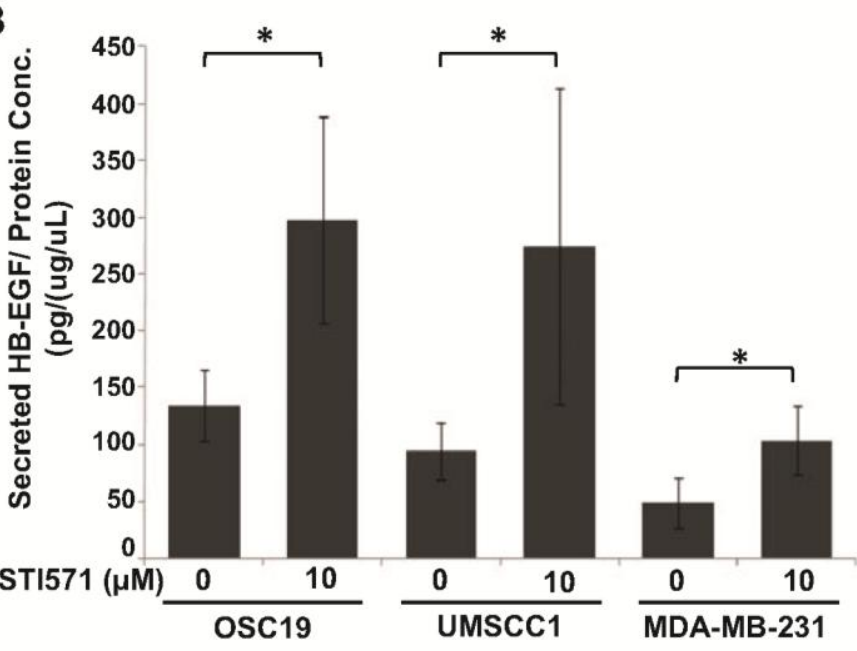

D

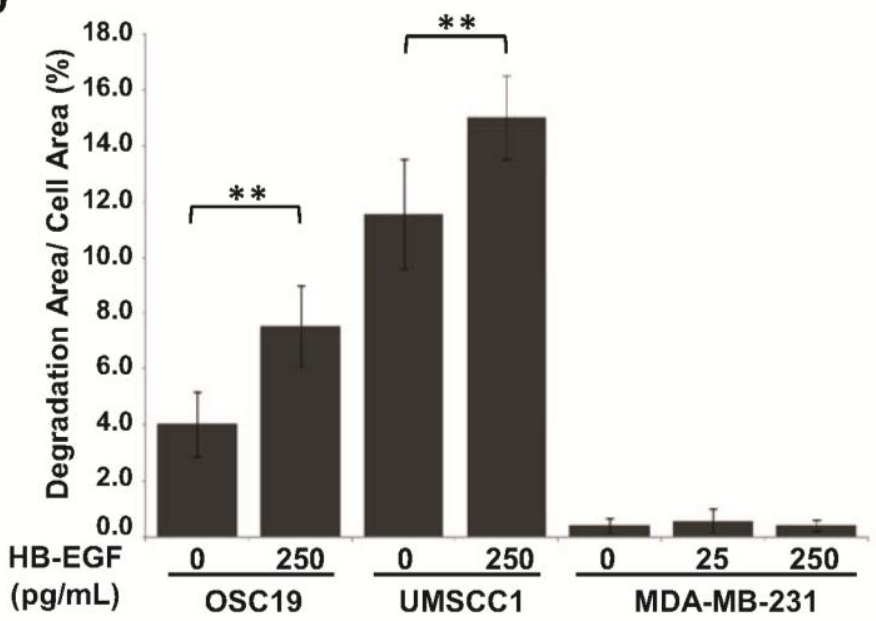

C
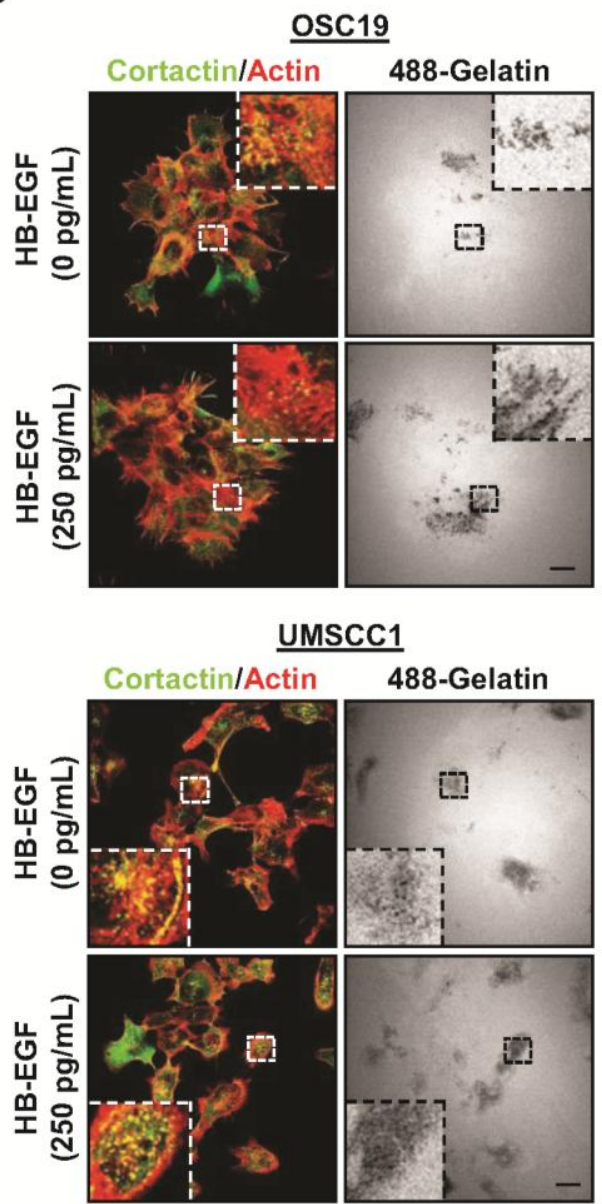

MDA-MB-231

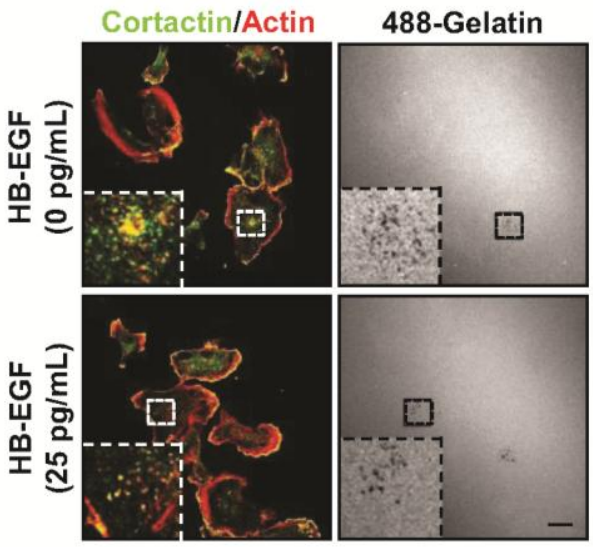

Hayes et al. Figure 6 
A

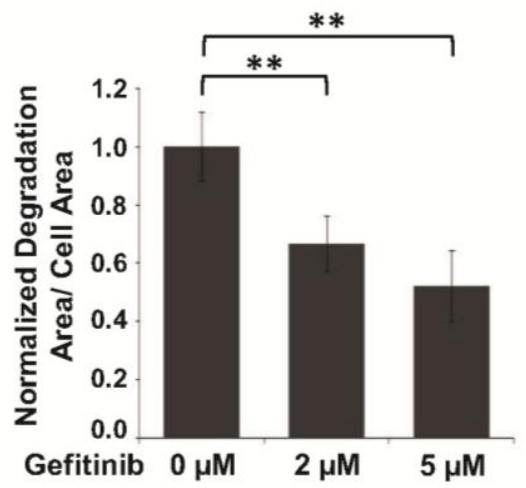

C

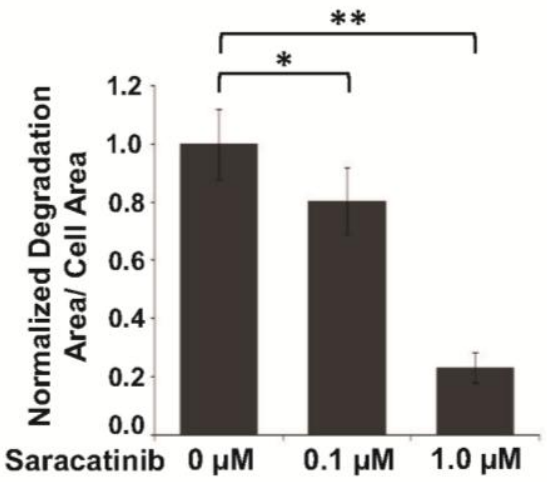

B

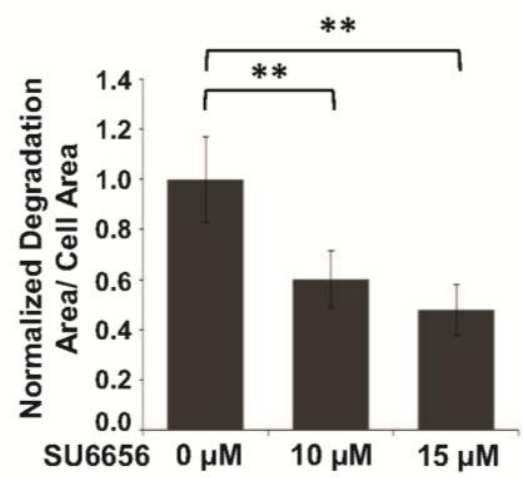

D

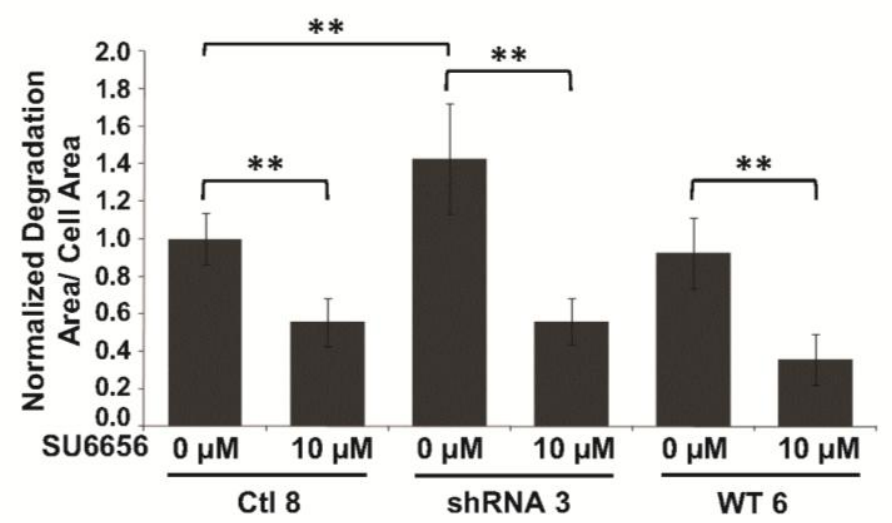

Hayes et al. Figure 7 
A

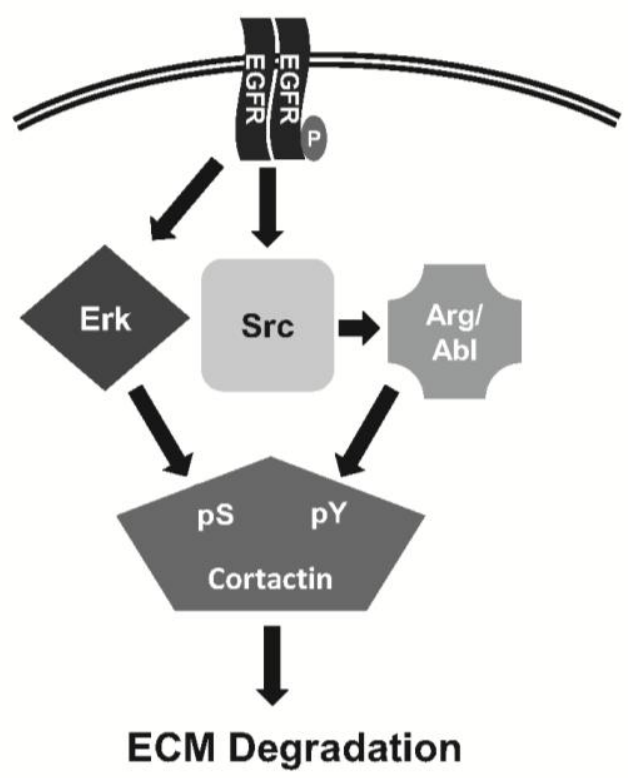

Hayes et al. Figure 8
B

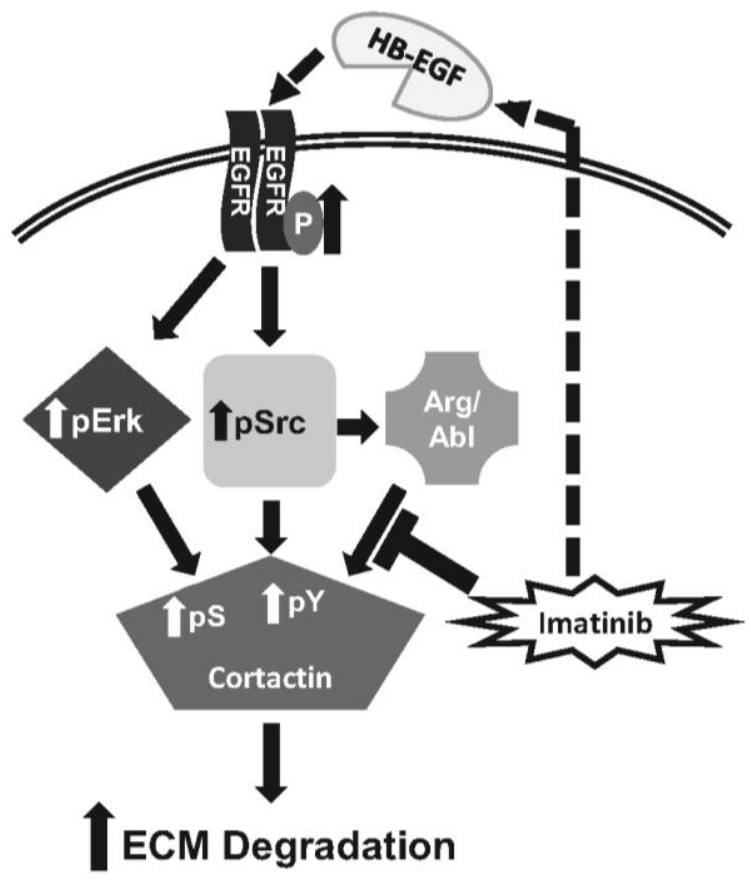




\section{Supplementary Figure Legends}

Supplementary Figure $1 \mathrm{Abl}$ knockdown does not impact the number of HNSCC invadopodia. (a) Src transformed 1483 cells transiently expressing empty vector (HACtl) or HA-tagged Abl (HA-Abl) are plated on Oregon Green 488-gelatin coated coverslips (pseudocolored white) for $24 \mathrm{~h}$, fixed and immunolabeled with anti-HA (blue), anti-cortactin (green), and rhodamine-phalloidin (F-Actin; red). Insets demonstrate areas of overlapping invadopodia marker localization with areas of gelatin clearing. Scale bars: $10 \mu \mathrm{m}$. Quantification of Abl knockdown on percentage of UMSCC1 cells degrading gelatin matrix (b) and invadopodia number per cell (c) from the assays conducted in Figure 1c. Data are represented as mean \pm C.I.

Supplementary Figure 2 Abl knockdown does not impact matrix degradation in MDAMB-231 cells. (a) MDA-MB-231 cells that were non-infected ( $\mathrm{NI}$ ) or infected with lentivirus containing control shRNA (Ctl), Abl specific (shRNA) or Abl shRNA with WT Abl re-expressed (WT) were plated on Oregon Green 488-gelatin coated coverslips (pseudocolored white) for $24 \mathrm{~h}$, fixed and labeled with anti-HA (blue), anti-cortactin (green) and rhodamine-phalloidin (F-Actin; red). Scale bars: $10 \mu \mathrm{m}$. (b) MDA-MB-231 cells infected as in (a) were lysed and $100 \mu \mathrm{g}$ of cell lysate analyzed for Abl expression of by Western blot analysis. Levels of $\beta$-actin were evaluated to verify equal protein loading. (c) Quantification of Oregon Green 488-gelatin degradation from MDA-MB-231 cells transfected as in (a). Error bars indicate mean percentages of gelatin degradation \pm C.I.

Supplementary Figure 3 Determination of PDGFR expression levels in OSC19, UMSCC1 and MDA-MB-231 cells. Total cell lysate $(150 \mu \mathrm{g})$ from each indicated line was assayed for PDGFRa expression by immunoblotting with anti-PDGFR a antibodies. $\mathrm{NIH} 3 \mathrm{~T} 3$ fibroblast lysate was included as a positive control. $\beta$-actin levels were assayed to verify equal protein loading.

Supplementary Figure $4 \mathrm{Abl}$ and Arg expression levels in wild-type (WT) and $\mathrm{Abl}^{-/-}$ ${ } \mathrm{Arg}^{-/-}$(DKO) mouse embryo fibroblasts (MEFs). (a) Western blot analysis of Abl and Arg protein levels in WT and DKO MEFs with anti-Abl and -Arg antibodies. $\beta$-actin 
levels were evaluated to validate equivalent protein loading. (b) DKO cells expressing GFP-tagged empty vector (Ctl), wild-type (WT), 527F, kinase inactive (K295M) Src, coexpressing 527F Src and wild-type (WT) Abl or wild-type (WT) MEFs expressing 527F Src were plated on Oregon Green 488-gelatin and assayed for invadopodia formation and matrix degradation for $12 \mathrm{~h}$. Cells were fixed and imaged by confocal microscopy for anti-GFP (3E6; blue) or anti-Abl (yellow), and co-labelled with anti-cortactin (green) and rhodamine-phalloidin (F-Actin; red). Boxed regions and insets denote comparable regions of invadopodia formation and gelatin degradation. Scale bars: $10 \mu \mathrm{m}$.

Supplementary Figure 5 Evaluation of imatinib on EGFR and Crk signaling. (a) Prolonged Western exposure of the EGFR pY1068 signal from the corresponding representative panel in Figure 5a showing EGFR activation. (b) UMSCC1 cells were treated with indicated concentration of DMSO $(0 \mu \mathrm{M})$, SU6656 and/or STI571 for $24 \mathrm{~h}$, lysed and assayed by Western blot analysis for Crk Y221 phosphorylation. Ratios of phosphorylated to total Crk levels are displayed. Parallel blots were probed for $\beta$-actin to confirm equal protein loading.

Supplementary Figure 6 Targeted inhibition of the EGFR-Src pathway. UMSCC1 cells treated with the indicated concentrations of gefitinib (a), SU6656 (b) and saracatinib (c) for $24 \mathrm{~h}$ were lysed and 25-50 $\mu \mathrm{g}$ total protein analyzed for drug efficacy by Western blot analysis with the indicated phosphorylation specific antibodies. (d) Western blot analysis of Src inhibition in UMSCC1 cells with manipulated Abl expression. Cell lysates $(50 \mu \mathrm{g})$ from UMSCC1 control (Ctl 8), shRNA Abl knockdown (shRNA 3) and Abl rescued shRNA cells (WT 6) treated with DMSO $(0 \mu \mathrm{M})$ or SU6656 $(10 \mu \mathrm{M})$ for $24 \mathrm{~h}$ were immunoblotted with anti-pY418Src and anti-Src. Parallel blots were probed for $\beta$-actin to confirm equal protein loading across all lines. Ratios of phosphorylated to total Src levels are shown.

Supplementary Figure 7 Saracatinib impairs invadopodia activity in HNSCC and MDA-MB-231 cells. Western blot analysis of 25-50 $\mu \mathrm{g}$ of protein from (a) OSC19 and (b) MDA-MB-231 cells treated with DMSO $(0 \mu \mathrm{M})$ or the indicated concentrations of saracatinib for $24 \mathrm{~h}$. Lysates were immunblotted for activation of EGFR (pY1068 EGFR), Src (pY418 Src) and Abl family kinases (pY221 Crk). Parallel blots were 
probed with antibodies against the non-phosphorylated protein forms as indicated. Ratios of phosphorylated/total protein relative to control for each analyzed protein and treatment condition are shown. (c) Effects of saracatinib on invadopodia activity. OSC19, UMSCC1 and MDA-MB-231 cells plated on Oregon Green 488-gelatin were treated with either DMSO $(0 \mu \mathrm{M})$ or the indicated saracatinib concentrations for $24 \mathrm{~h}$. Cells were fixed and labeled with rhodamine-phalloidin and anti-cortactin antibodies. Cells and gelatin matrix were and imaged by confocal microscopy. The percentage of gelatin degradation per cell area calculated relative to controls. Data are represented as mean \pm C.I.; ${ }^{*} P \leq 0.01 ;{ }^{*} P \leq 0.05$. 
A

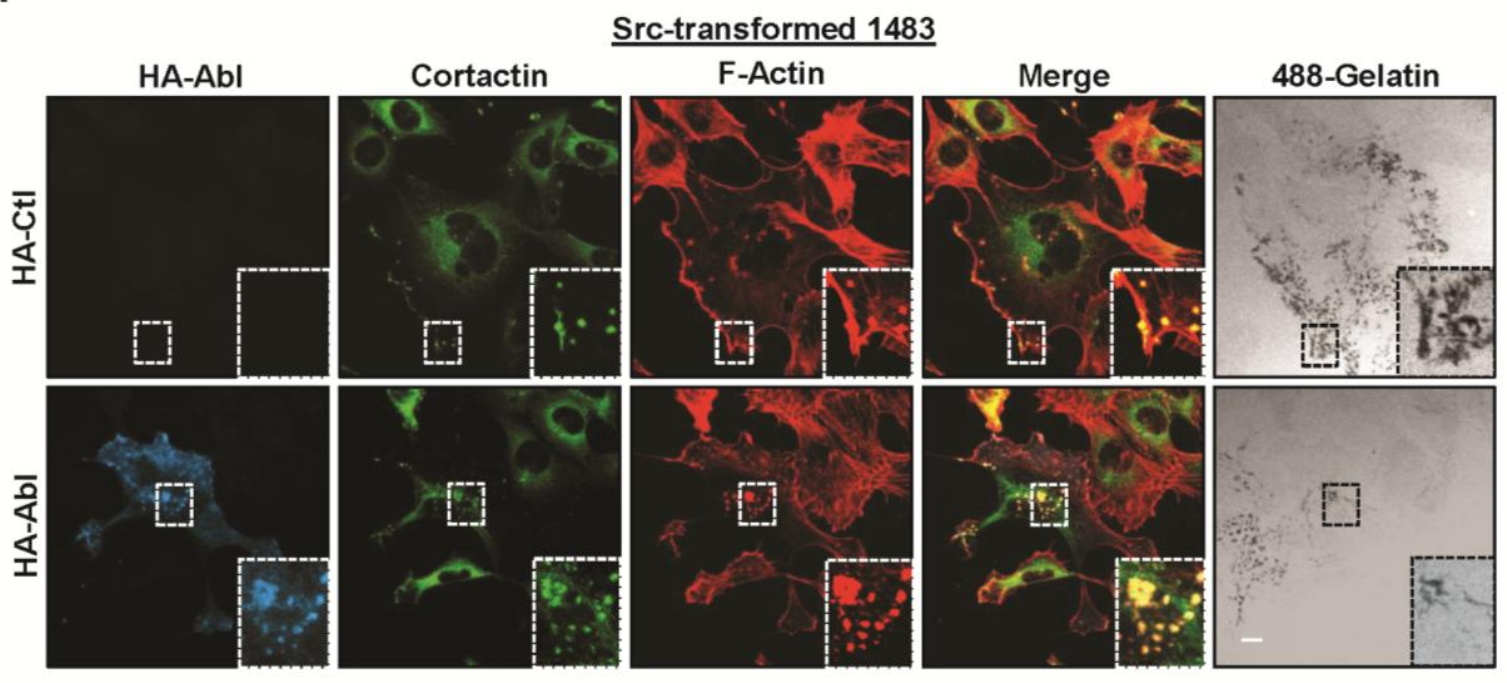

B

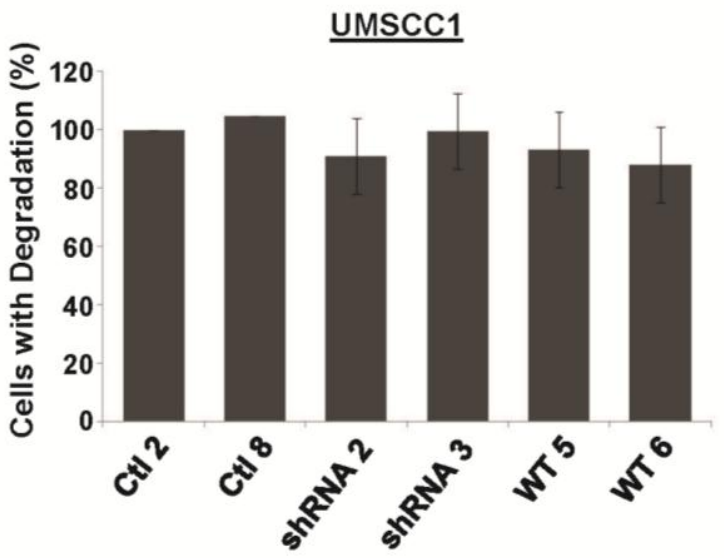

C

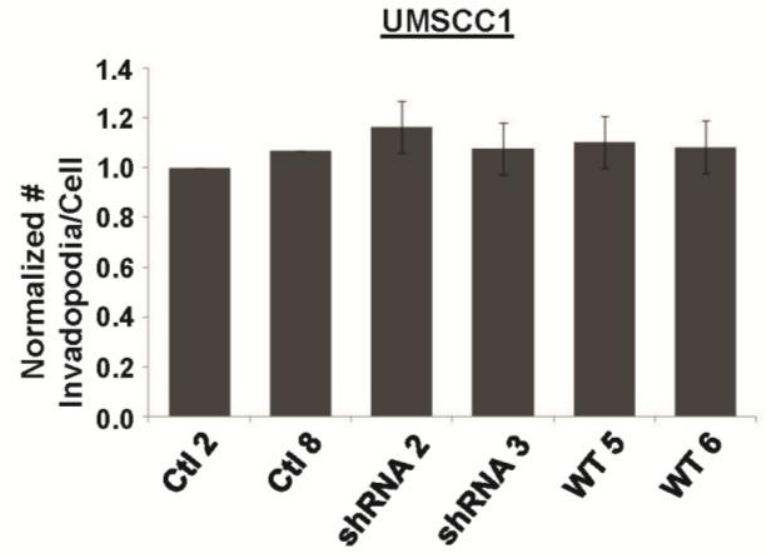

Hayes et al. Supplementary Figure 1 
A

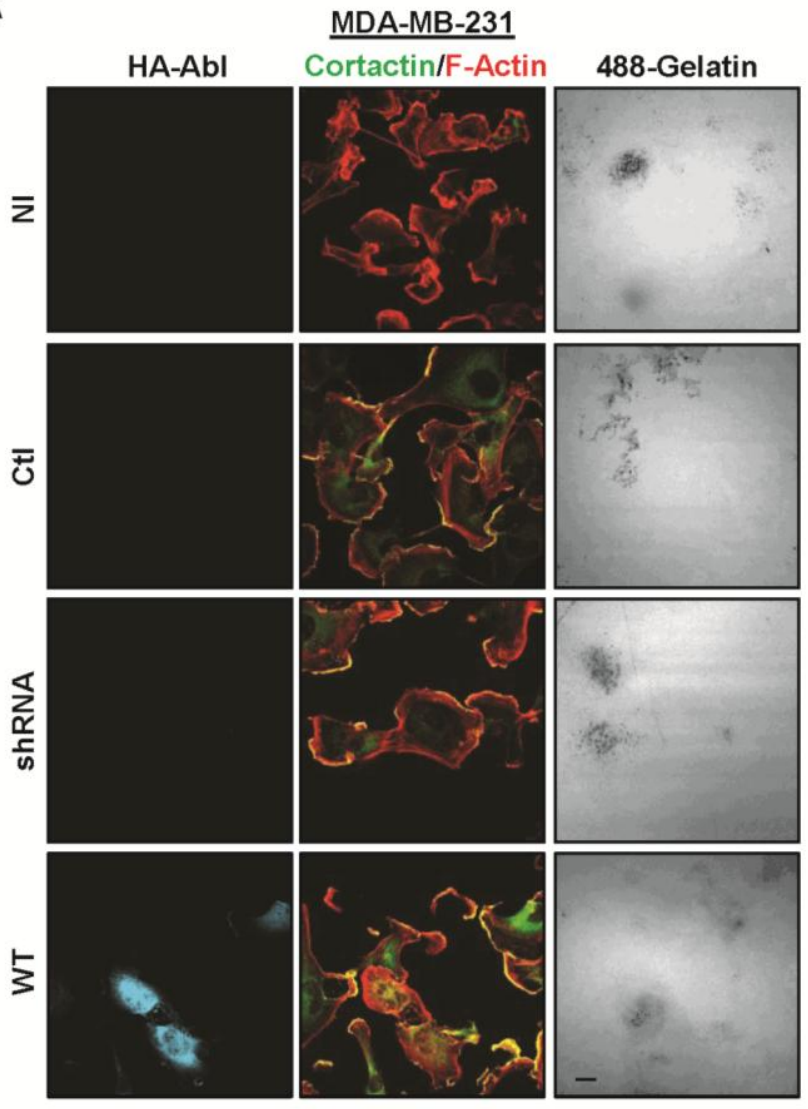

B

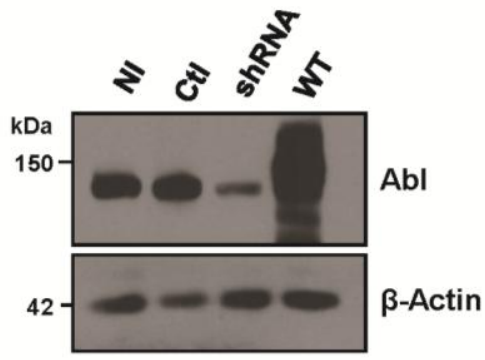

C

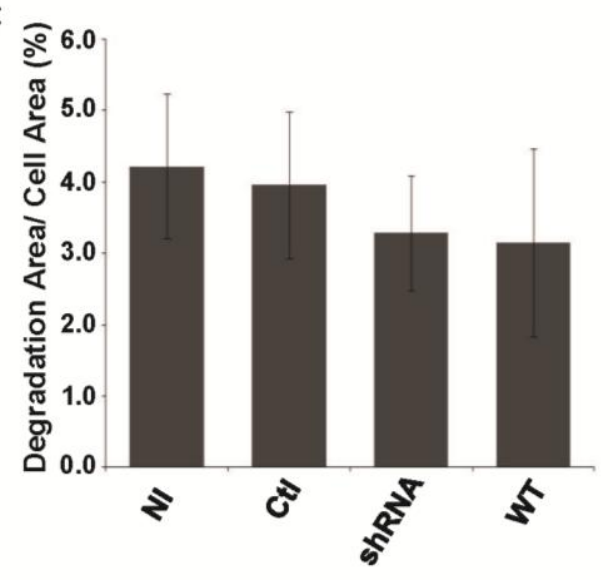

Hayes et al. Supplementary Figure 2 


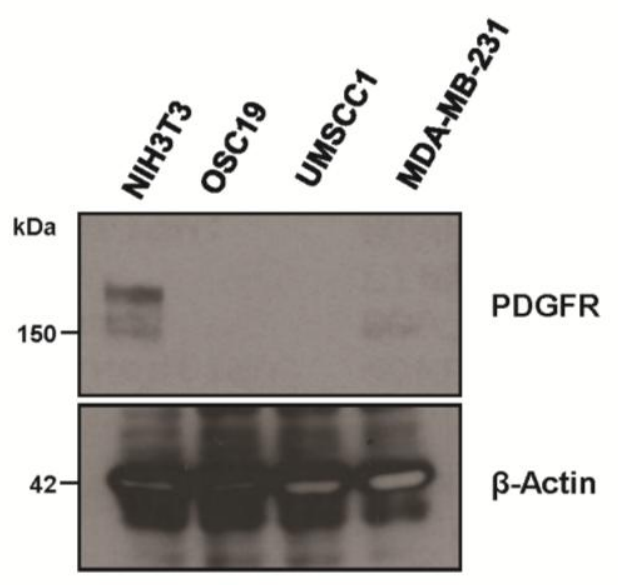

Hayes et al. Supplementary Figure 3 
A

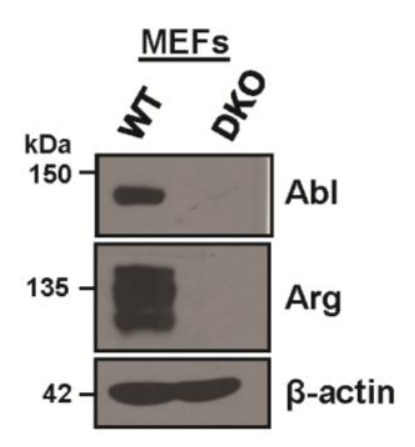

B

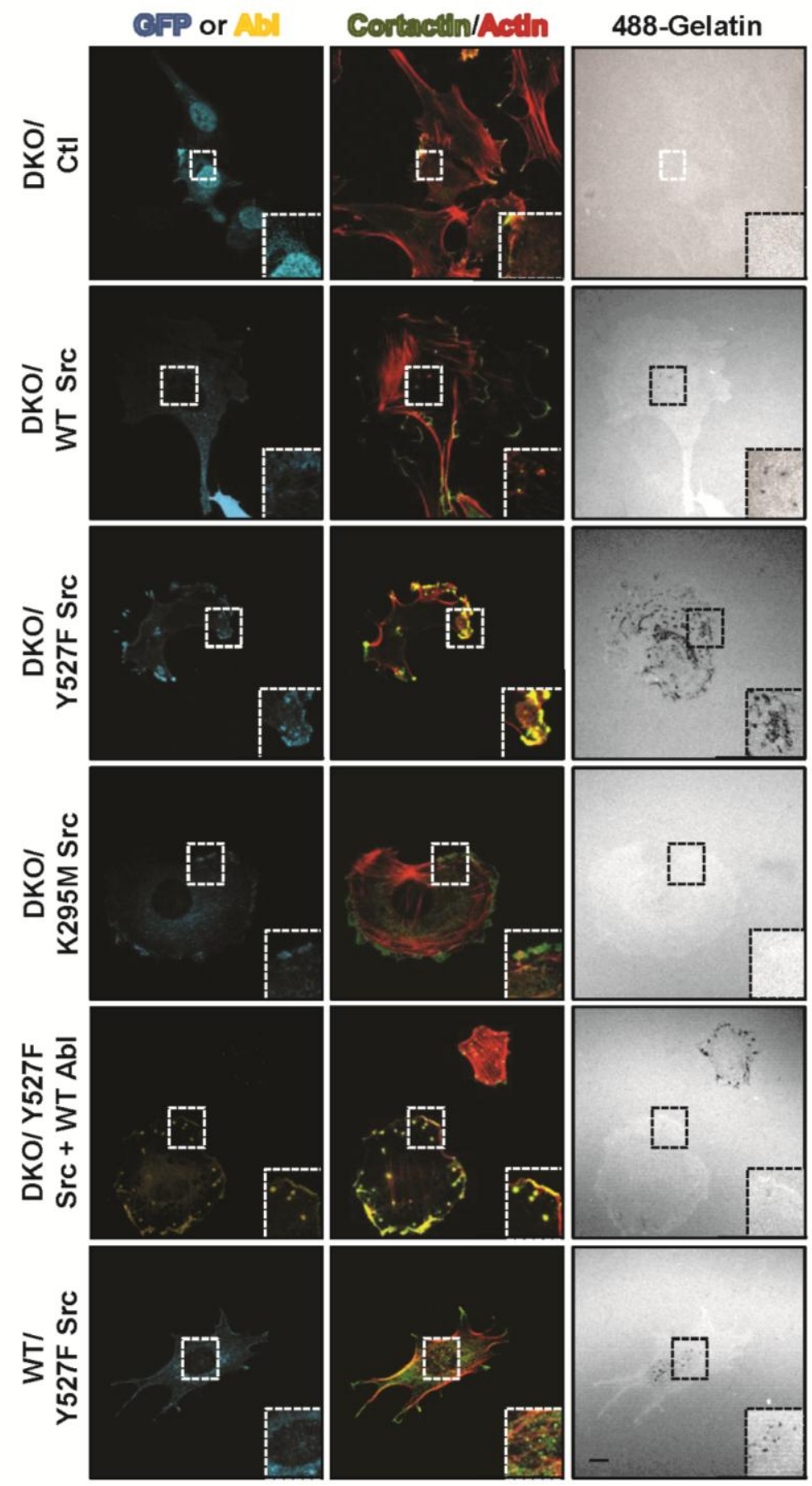

Hayes et al. Supplementary Figure 4 
A

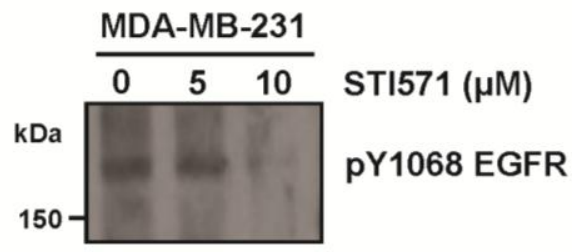

B

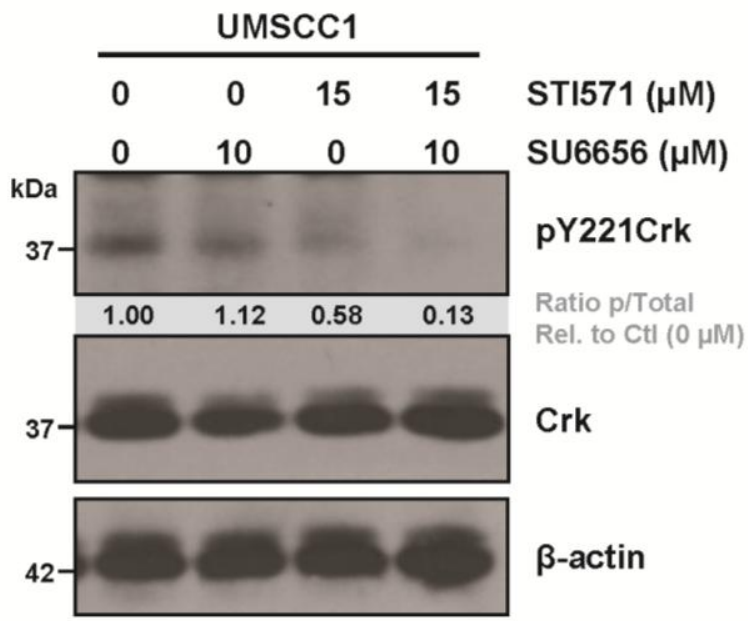

Hayes et al. Supplementary Figure 5 
A

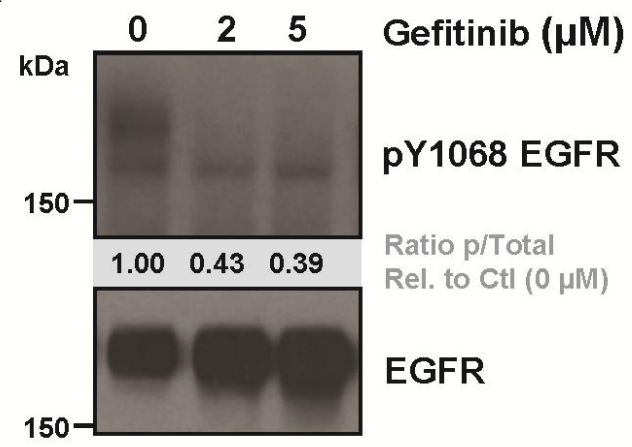

B

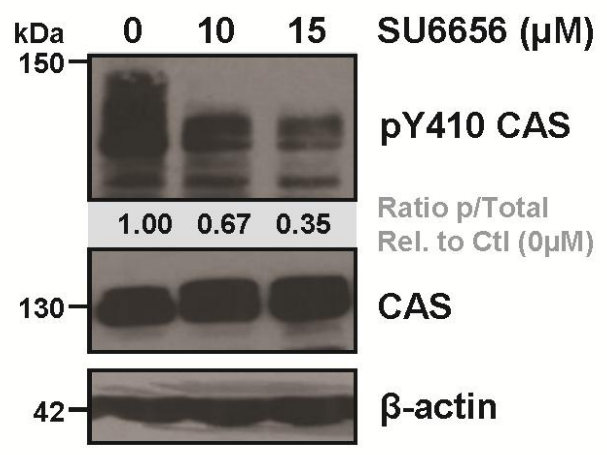

C
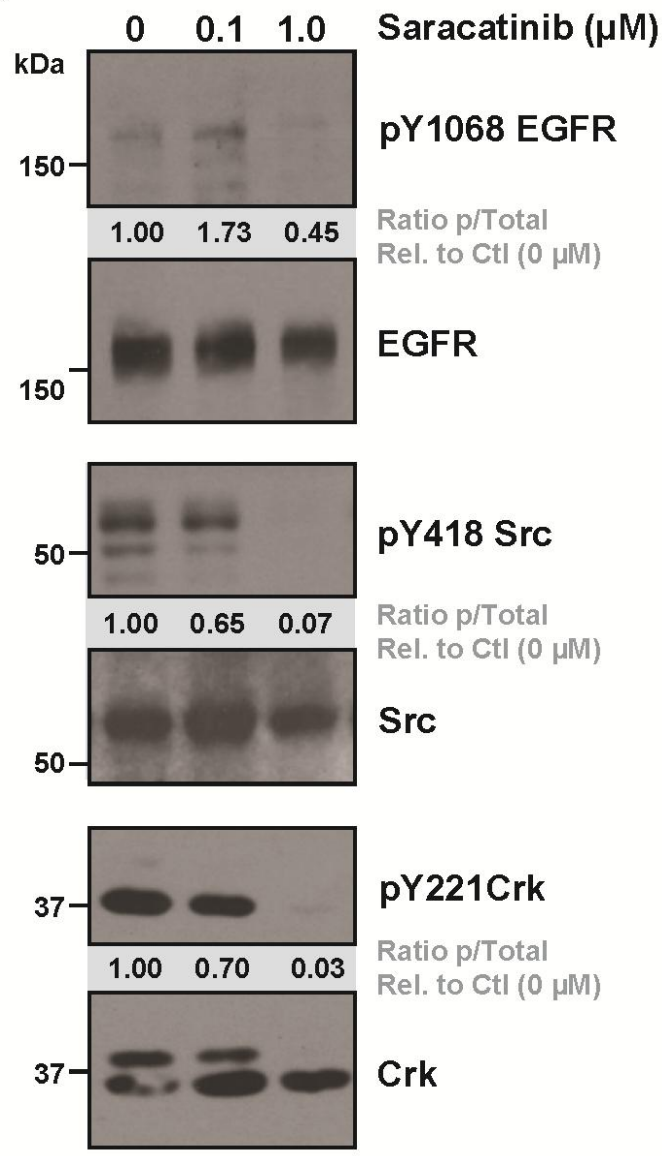

D

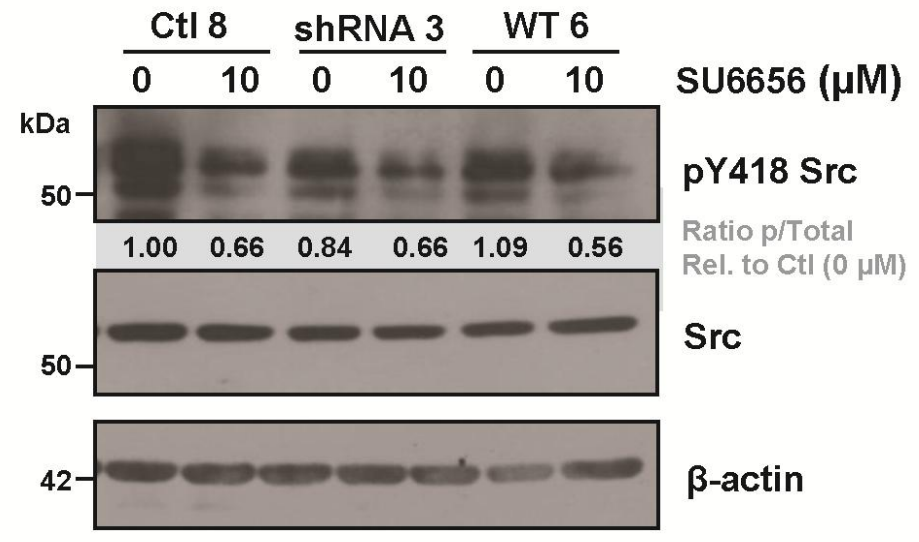

Hayes et al. Supplementary Figure 6 
A

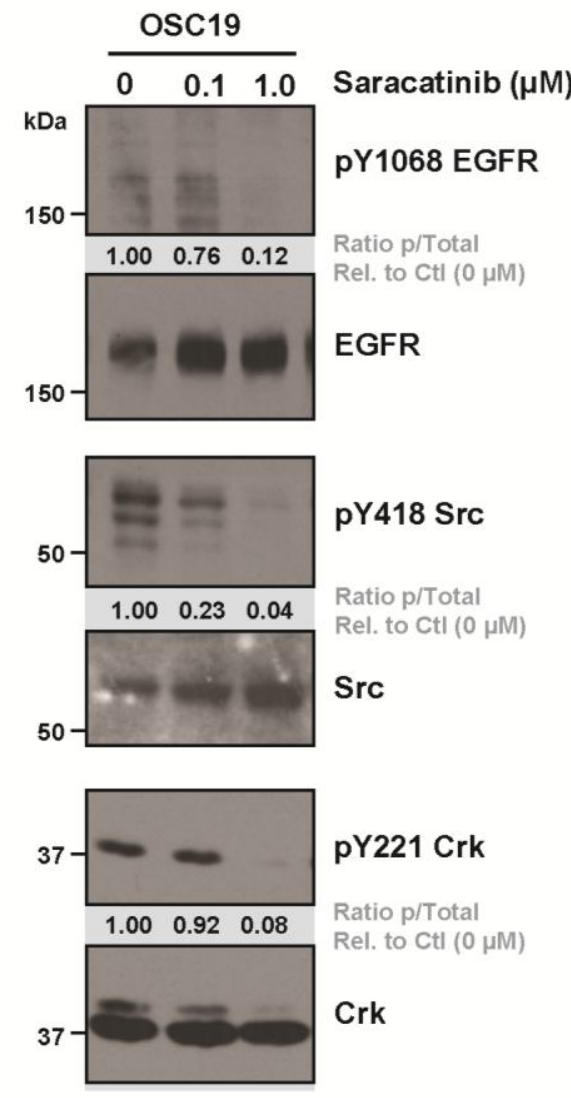

B

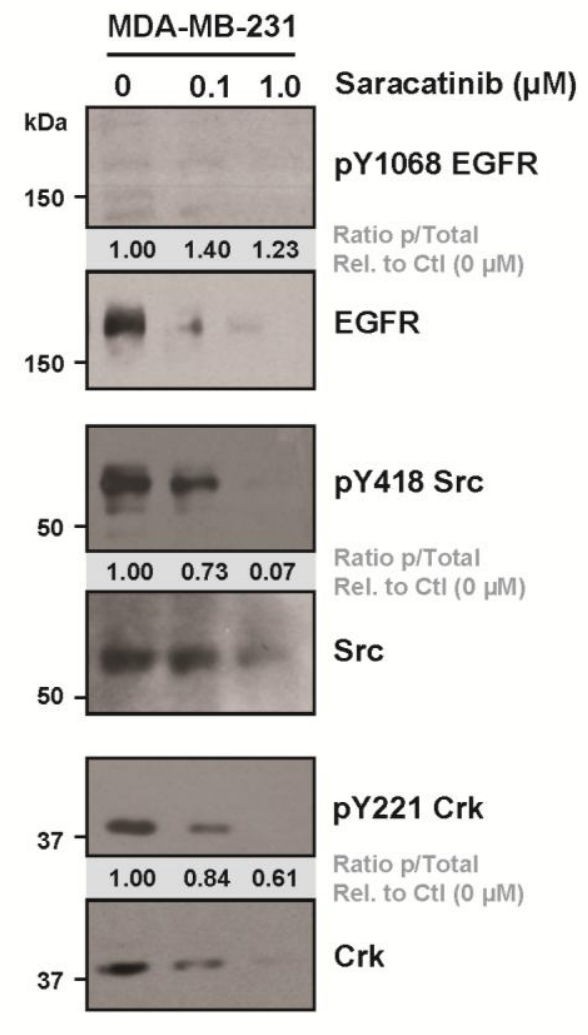

C

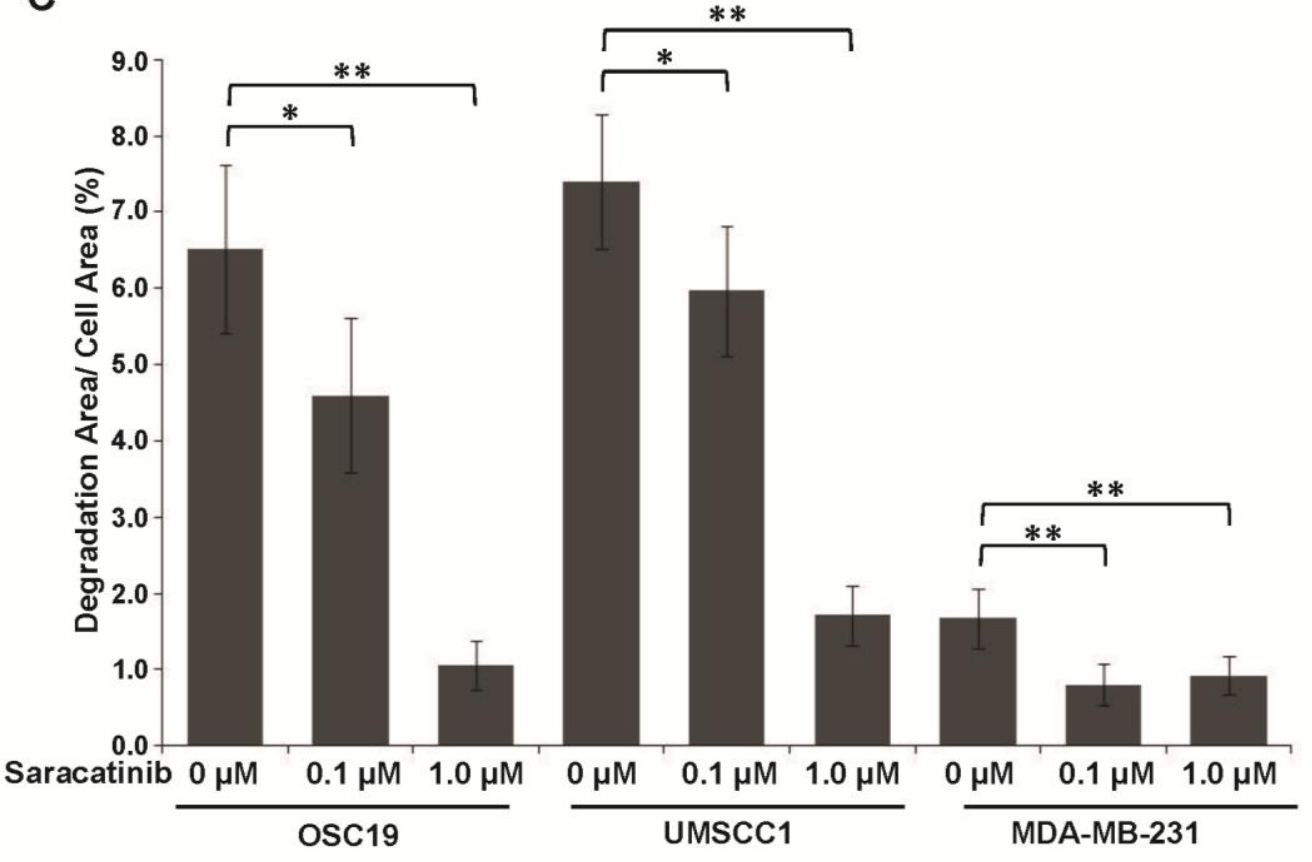

Hayes et al. Supplementary Figure 7 


\title{
Study 2: Saracatinib Impairs Head and Neck Squamous Cell Carcinoma Invasion by Disrupting Invadopodia Function
}

\author{
Amanda Gatesman Ammer ${ }^{1,4}$, Laura C. Kelley ${ }^{1,4}$, Karen E. Hayes ${ }^{1,4}$, Jason V. Evans ${ }^{1}$, \\ Lesly Ann Lopez-Skinner ${ }^{1,4}$, Karen H. Martin ${ }^{1}$, Barbara Frederick ${ }^{2,4}$, Brian L. \\ Rothschild ${ }^{2}$, DavidRaben ${ }^{2}$, Paul Elvin ${ }^{3}$, Tim P. Green ${ }^{3}$ and Scott A. Weed ${ }^{1}$
}

${ }^{1}$ Department of Neurobiology and Anatomy, Program in Cancer Cell Biology, Mary Babb Randolph Cancer Center, West Virginia University, Morgantown, West Virginia, 265069300 ,

${ }^{2}$ Department of Radiation Oncology, Anschutz Medical Campus, University of Colorado Denver, Aurora, CO, 80045 and 3 AstraZeneca Pharmaceuticals, Alderley Park, Cheshire, United Kingdom

Published in J Cancer Sci Ther. 2009 Nov 30;1(2):52-61.

Corresponding author:

Scott A. Weed

West Virginia University

Mary Babb Randolph Cancer Center

Morgantown, WV, 26508-9300

Phone: 304-293-3016

Fax: 304-293-4667

E-mail: sweed@hsc.wvu.edu

${ }^{4}$ Note: These authors contributed equally to this manuscript

Running title: Saracatinib inhibits invadopodia activity 


\section{Abstract}

Elevated Src kinase activity is linked to the progression of solid tumors, including head and neck squamous cell carcinoma (HNSCC). Src regulates HNSCC proliferation and tumor invasion, with the Src-targeted small molecule inhibitor saracatinib displaying potent anti-invasive effects in preclinical studies. However, the pro-invasive cellular mechanism(s) perturbed by saracatinib are unclear. The anti-proliferative and antiinvasive effects of saracatinib on HNSCC cell lines were therefore investigated in preclinical cell and mouse model systems. Saracatinib treatment inhibited growth, cell cycle progression and transwell Matrigel invasion in HNSCC cell lines. Dose-dependent decreases in Src activation and phosphorylation of the invasion-associated substrates focal adhesion kinase, p130 CAS and cortactin were also observed. While saracatinib did not significantly impact HNSCC tumor growth in a mouse orthotopic model of tongue squamous cell carcinoma, impaired perineural invasion and cervical lymph node metastasis was observed. Accordingly, saracatinib treatment displayed a dosedependent inhibitory effect on invadopodia formation, extracellular matrix degradation and matrix metalloprotease 9 activation. These results suggest that inhibition of Src kinase by saracatinib impairs the pro-invasive activity of HNSCC by inhibiting Src substrate phosphorylation important for invadopodia formation and associated matrix metalloprotease activity.

Keywords: Saracatinib, Src, Head and Neck cancer, invadopodia, invasion, MMP 


\section{Abbreviations}

CAS- Crk-associated substrate

c-Src- cellular Src kinase

ECM- extracellular matrix

FACS- fluorescence-activated cell sorting

FAK- focal adhesion kinase

FITC- fluorescein isothiocyanate

HNSCC- head and neck squamous cell carcinoma

IHC- immunohistochemistry

SFK- Src family kinase 


\section{Introduction}

Tumor cell invasion and metastasis is a compounding problem in cancer management, with therapeutic intervention of tumor invasion becoming recognized as an increasingly relevant clinical factor (Dolgin, 2009). Increased activation of the proto-oncogene c-Src (Src) has been established in enhancing tumor progression in human cancer and corresponds with poor clinical outcome (Irby \& Yeatman, 2000; Yeatman, 2004). Src is responsible for governing signaling pathways that regulate proliferation, angiogenesis, resistance to apoptosis, adhesion, motility and invasion (Summy \& Gallick, 2006). High Src expression and/or activity is observed in metastases, supporting a role for $\mathrm{Src}$ in tumor progression by enhancing tumor invasion and metastatic potential (Summy \& Gallick, 2003; Yeatman, 2004). Small molecules targeting Src kinase activity suppress proliferation, invasion and metastasis in preclinical settings (Summy \& Gallick, 2006), and are currently being evaluated in clinical trials (Kopetz et al., 2007).

Head and neck squamous cell carcinoma (HSNCC) is highly invasive, frequently metastasizing to cervical lymph nodes and corresponds with poor prognosis (Kramer et al., 2005). Src overexpression is common in HNSCC (van Oijen et al., 1998) and is activated following engagement of the epidermal growth factor receptor (EGFR), where it modulates HNSCC growth and invasion through several signaling pathways (Zhang et al., 2004). The small molecule Src kinase inhibitor dasatinib suppresses motility and invasion of HNSCC cells in vitro and in mouse xenografts models, corresponding with decreased Src activation and invasion-associated substrate phosphorylation (Johnson et al., 2005; Sen et al., 2009). Amplification and/or overexpression of Src substrates in HNSCC correlates with poor clinical outcome, potentially serving to magnify Src pathway effects on HNSCC invasion and metastasis (Kelley et al., 2008).

HNSCC invasion and metastatic spread is mediated in part by the action of matrix metalloproteases (MMPs), with MMP1, MMP2, MMP9 and MT1-MMP activity associated with poor outcome (Rosenthal \& Matrisian, 2006). MT1-MMP, MMP2 and MMP9 localize to invadopodia, actin-based ventral protrusions in invasive tumor cells that mediate focalized proteolysis of the extracellular matrix (ECM) (Linder, 2007; Weaver, 2006). Invadopodia formation is dependent on Src activity, which enhances 
MMP2 and MMP9 secretion (Hsia et al., 2003; Mueller et al., 1992), and matrix degradation in HNSCC cells (Clark et al., 2007). The collective localization and action of MMPs at invadopodia allows matrix remodeling to accommodate primary tumor growth and to allow dissemination of encapsulated tumor cells to local and distant sites (Gimona et al., 2008).

Saracatinib (AZD0530) is a recently developed anilinoquinazoline inhibitor designed to disrupt Src kinase activity (Hennequin et al., 2006; Summy \& Gallick, 2006). Saracatinib exhibits inhibitory effects on tumor growth in some model systems (Herynk et al., 2006), but several preclinical reports suggest that the primary anticancer effects of saracatinib are impaired tumor cell migration and invasion in HNSCC and other cancer types (Green et al., 2009; Koppikar et al., 2008; Nozawa et al., 2008). The anti-invasive effects of saracatinib are consistent with the effects of Src kinase inhibition in HNSCC by dasatinib, another Src-targeted inhibitor (Johnson et al., 2005). Saracatinib is currently being evaluated in phase $\mathrm{I} / \mathrm{I}$ clinical trials for efficacy against advanced stage HNSCC and other tumor types (Kopetz et al., 2007).

Although saracatinib and other Src inhibitors are effective anti-invasive compounds, a complete understanding of the how therapeutic Src inhibition perturbs tumor invasion at the cellular level is lacking. We show that saracatinib inhibited Src activation and phosphorylation of the invadopodia regulatory proteins focal adhesion kinase (FAK), p130 Crk-associated substrate (CAS) and cortactin in HNSCC cells. Saracatinib suppressed HNSCC growth and cell cycle progression in a subset of HNSCC cell lines. Administration of saracatinib to nude mice containing orthotopic HNSCC tongue tumors inhibited Src activity, cortactin phosphorylation, perineural invasion and lymph node metastasis. We also demonstrated that saracatinib prevented invadopodia formation and ECM degradation in invasive HNSCC cells, as well as secretion and activation of MMP9. Collectively these results suggest that saracatinib exhibits anti-tumor effects in HNSCC by inhibiting invasion through the prevention of invadopodia formation. The ability of saracatinib to prevent invadopodia-mediated ECM proteolysis reveals a cellular process perturbed by Src inhibitors that is likely utilized in the progression of HNSCC and other invasive carcinomas containing high Src activity. 


\section{Materials and Methods}

\section{Cell lines, antibodies and Western blotting}

HNSCC cell lines 1483, HN31, UMSCC 1, UMSCC19 and MSK 921 were maintained as described (Rothschild et al., 2006). Western blotting of cell lysates was conducted essentially as before (Rothschild et al., 2006). Western blotting of secreted MMP2 and 9 was conducted on conditioned media, with volumes adjusted to compensate for variations in cell numbers using dimethyl sulfoxide (DMSO)-treated cell numbers as controls.

Antibodies for immunoblotting included anti-Src (1:1000; Santa Cruz Biotechnology, Santa Cruz, CA, USA), anti-pY418 Src (1:1000; Invitrogen, Carlsbad, CA, USA), antip130CAS (1:1000; BD Biosciences, San Jose, CA, USA), anti-pY410 p130CAS (1:1000; Cell Signaling Technology, Danvers, MA, USA), anti-FAK (1:1000; BD Biosciences), anti-pY861 FAK (1:1000; Invitrogen) and anti-cortactin $(1 \mu \mathrm{g} / \mathrm{ml} ; 4 \mathrm{~F} 11$ (Rothschild et al., 2006)). For detection of human pY421 cortactin, a custom antibody was developed by 21st Century Biochemicals (Marlboro, MA, USA). Briefly, a synthetic cortactin peptide encompassing the sequence around tyrosine 421 (NH2VpYEDAASFKL-COOH) was synthesized, phosphorylated and injected into rabbits. Immune serum was passed through a column containing agarose beads coupled to the equivalent non-phosphorylated peptide, and then passed over a second agarose column containing a partially overlapping phosphorylated peptide $\left(\mathrm{NH}_{2}-\right.$ LPSSPVPYEDAA-COOH). Bound antibodies were eluted, concentrated and screened for specificity by Western blotting against recombinant cortactin mutant proteins harboring phenylalanine-tyrosine point mutations at tyrosine 421 (Fig. 1, Supplemental Material). Anti-ERK1/2 (Cell Signaling) and anti-pT202/pY204 ERK1/2 (Cell Signaling) were used at 1:1000. Anti-MMP2 (1:500; Millipore, Billerica, MA, USA) was used to detect cellular MMP2 levels. Secreted MMP2 was detected with antibody CA-4001 (1:100; Millipore). Cellular and secreted MMP9 was detected with monoclonal antibody 9D4.2 (1:100; Millipore). All Western blots were quantified by densitometry and ImageJ analysis, and band intensities determined relative to non-treated controls. 


\section{Cell proliferation and cell cycle progression assays}

For cell proliferation assays, 4,000 cells were seeded overnight and treated with 0-10 $\mu \mathrm{M}$ saracatinib (AstraZeneca, Cheshire, UK) for $5 \mathrm{~d}$. $100 \mu \mathrm{g}$ of 3-(4,5-dimethylthiazol-2yl)-2,5-diphenyltetrazolium bromide (Sigma-Aldrich, St. Louis, MO, USA) was added to each saracatinib treatment condition for $4 \mathrm{~h}$, cells were washed and the reduced dye extracted with a $75 \%$ isopropanol/2\% $\mathrm{HCl} / 23 \% \mathrm{H} 2 \mathrm{O}$ mixture. Dye absorbance was read at $490 \mathrm{~nm}$ with an automated plate reader.

Cell cycle distribution was determined by fluorescence activated cell sorting (FACS) as previously described (Frederick et al., 2007).

\section{Invasion assays}

BioCoat Matrigel invasion chambers (BD Biosciences) were rehydrated with serum-free DMEM media for 2 h. $1 \times 105$ cells suspended in serum-free media were plated in the chamber insert and incubated for $2 \mathrm{~h}$ to allow attachment. The media in the upper and lower chambers was replaced, with serum-free DMEM added to the upper chamber and DMEM containing $5 \%$ FBS added to the lower chamber to generate a chemotactic gradient. Increasing doses of saracatinib $(0-1 \mu \mathrm{M})$ of saracatinib as indicated (Fig. 1D) to the upper and lower chambers. Cells were allowed to invade for $12-24 \mathrm{~h}$ (depending on cell line), fixed with $10 \%$ buffered formalin phosphate (Fisher Scientific, Hanover Park, IL, USA) and rinsed with PBS. Non-invasive cells were removed from the interior of the chamber insert with a swab and the remaining cells were stained with $0.4 \%$ Crystal Violet solution (Fisher) for 15 min. Invasion was assessed by counting cells in four random 20X microscopic fields.

\section{Orthotopic xenograft assay of HNSCC invasion}

An in vivo mouse model of oral tongue squamous cell carcinoma was established as described (Myers et al., 2002) with minor modification using female athymic Foxn $1^{\text {nu/nu }}$ mice 4-5 weeks of age (Harlan Laboratories, Indianapolis, IN, USA). All animal procedures were conducted according to an approved protocol by the West Virginia University Animal Care and Use Committee. Anesthetized mice were injected with $2.5 \mathrm{x}$ 
$10^{4}$ UMSCC 1 cells suspended in DMEM into the anterior $\sim 1 / 3$ of the tongue. After $10 \mathrm{~d}$, treatment was initiated by daily oral gavage of $25 \mathrm{mg} / \mathrm{kg}$ saracatinib suspended in a sterile solution of $0.5 \%$ methyl cellulose/ $0.1 \%$ polysorbate 80 (Tween 80 , SigmaAldrich). Control animals were gavaged with the methylcellulose/Tween 80 vehicle. No overt difficulties were encountered when gavaging mice over time as the tumor size increased. Six mice were used for each treatment group. After $30 \mathrm{~d}$ of treatment, mice were euthanized by carbon dioxide inhalation and tumor volumes determined as described (Huang et al., 2002). Tongues, sublingual tissue containing the superficial cervical lymph nodes and the tracheoesophageal region, deep cervical and mediastinial lymph nodes, liver and lung were removed from each animal, rinsed, fixed and paraffin embedded for routine histological evaluation.

\section{Immunohistochemistry}

Human HNSCC cases were obtained from the West Virginia Tissue Bank and used under the approval of West Virginia University Institutional Review Board. Fivemicrometer sections from human HNSCC and mouse tissue blocks were processed and immunolabeled or hematoxylin and eosin (H\&E) stained using a Discovery XT automated staining system (Ventana Medical Systems, Tucson, AZ, USA). For immunohistochemistry, primary antibody conditions were: anti-total Src (Cell Signaling) 1:600 in Dako diluent (Dako, Carpinteria, CA, USA) for $1 \mathrm{~h}$, anti-pY416 Src family kinase (SFK) (Cell Signaling) 1:25 in phosphate saline solution (PSS) (Ventana) for 12 h, anti-total cortactin (Novus Biologicals, Littleton, CO, USA) 1:700 in PSS for $1 \mathrm{~h}$, antipY421 cortactin 1:50 in Tris-buffered saline containing 4\% BSA for $1 \mathrm{~h}$, and prediluted anti-cytokeratin 14 (Abcam, Cambridge, MA, USA) for $20 \mathrm{~min}$. Primary antibodies were detected using the Omnimap antibody horseradish peroxidase kit (Ventana) and slides were counterstained with hematoxylin. Images were acquired as described (Rothschild et al., 2006). For quantifying pY416 Src and pY421 cortactin staining intensities, brightfield images from at least 5 randomly selected images were captured on an Olympus ZX70 Provis microscope (Olympus, Center Valley, PA, USA) with a 20x/0.70 UPlanApo objective and an Optronics MicroFire 1600x1200 color CCD camera (Optronics Inc, Goleta, CA, USA) using the Stereolnvestigator imaging package 
(MBF Bioscience, Williston, VT) with the same camera settings and brightfield correction enabled to ensure even illumination across the image. Brown 3,3'diaminobenzidine (DAB) staining was separated from blue hematoxylin staining using the color deconvolution plug-in function of ImageJ $(\mathrm{NIH})$ as described (Park et al., 2008). The vector values for the $D A B$ staining were determined from ROls with brown staining $(R=0.425, G=0.600$ and $B=0.677$ for $p Y 421$ cortactin; $R=0.475, G=0.653$ and $\mathrm{B}=0.686$ for $\mathrm{pY} 416 \mathrm{Src}$ ). Brown images were inverted and intensities were measured inside the tumor tissue. The mean DAB intensities were averaged within the group to calculate ratios of phosphorylation-specific staining in treated vs. control tissues.

\section{Immunofluorescence labeling, confocal microscopy and image analysis}

UMSCC1 cells were plated on fluorescein isothiocyanate (FITC)-gelatin (Sigma) coated coverslips as described (Artym et al., 2006) for $2 \mathrm{~h}$. Cells were left untreated or treated with saracatinib for $6 \mathrm{~h}$, rinsed and fixed with $4 \%$ formaldehyde. Cells were permeabilized with $0.4 \%$ Triton-X/PBS for 4 minutes, then blocked in 5\% BSA/PBS for 1 h. To identify invadopodia, cells were incubated with rhodamine-conjugated phalloidin (1:1000; Invitrogen), anti-cortactin monoclonal antibody 4F11 $(1 \mu \mathrm{g} / \mathrm{ml})$ and polyclonal pTyr-100 (1:200; BD Biosciences) in 5\% BSA/PBS for $1 \mathrm{~h}$. After washing, cells were incubated in 5\% BSA/PBS containing AlexaFluor 405 goat anti-rabbit and AlexaFluor 647 goat anti-mouse secondary antibodies (Invitrogen) at 1:1000. Cells were rinsed and mounted in Fluoromount-G (SouthernBiotech, Birmingham, AL, USA).

For quantifying saracatinib effects on invadopodia incidence and matrix degradation, eight-bit $1024 \times 1024$ pixel confocal images were acquired with a Zeiss LSM510 confocal microscope using AIM software (Carl Zeiss Microlmaging, Thronwood, NY, USA). Images were scanned with a 63x/1.4 NA Oil Plan-Apochromat objective at 1.3x zoom, yielding a resolution of 10.14 pixels $/ \mu \mathrm{m}$. All images of the FITC-gelatin were taken with the same parameters (pinhole size, laser intensity and gain) so image intensity would be comparable between samples. For invadopodia formation, a minimum of six independent fields comprising $>50$ cells were analyzed for cells containing invadopodia compared to total cell number. For matrix degradation, cells were analyzed using ImageJ software. Actin images were adjusted to threshold values to include all cellular 
regions, and the resulting images were used to calculate total cell areas in $\mu \mathrm{m}^{2}$. For quantifying matrix degradation, FITC-gelatin images were inverted so that regions with increased degradation would yield higher intensity values, ensuring selection of all areas of matrix degradation. The integrated density was reported as the amount of degradation per total cell area. A minimum of 15 cells was analyzed for each saracatinib concentration.

\section{Gelatin zymography}

UMSCC1 and 1483 cells were plated overnight at $5 \times 10^{6}$ in complete media and were treated with saracatinib at increasing dosage for $24 \mathrm{~h}$, rinsed and incubated for $24 \mathrm{~h}$ in serum-free media containing the equivalent saracatinib dose. Cells were counted, and conditioned media collected and concentrated by ultrafiltration using Amicon Ultra-4 centrifugal filter devices with a 10kDa molecular weight cutoff (Millipore). Zymography was conducted as described (Clark et al., 2007) with minor modification. Conditioned media (35 $\mu \mathrm{l}$ ) was diluted in 2X non-reducing SDS-PAGE sample buffer and resolved on $8 \%$ SDS-PAGE gels containing $1 \mathrm{mg} / \mathrm{ml}$ bovine gelatin (Sigma). Aliquots of serum-free DMEM and DMEM containing 10\% FBS were used as negative and positive controls, respectively. MMP activity was renatured by washing gels in $2.5 \%$ Triton X-100 for 30 min, followed by washing gels in Developing Buffer (50 mM Tris, $0.2 \mathrm{M} \mathrm{NaCl}, 5 \mathrm{mM}$ $\mathrm{CaCl} 2,0.02 \% \mathrm{Brij} 35)$ for $30 \mathrm{~min}$ at room temperature. Gels were then incubated in renewed Developing buffer for $24 \mathrm{~h}$ at $37^{\circ} \mathrm{C}$ to allow MMP activity to proceed. Gels were stained with Coomassie Brilliant Blue R-250 (0.5\% w/v) for $30 \mathrm{~min}$, followed by destaining in water. Resulting gels were scanned using a FotoAnalyst Investigator (Fotodyne Inc, Hartland, WI, USA) and areas of gelatinase activity quantified using ImageJ. Results were adjusted relative to control DMSO treatment for gelatinase activity and cell counts for each treatment to compensate for variations in final cell numbers.

\section{Statistical analysis}

Differences in mean values between saracatinib treatment groups for invasion, invadopodia and gelatinase assays were evaluated using one-way ANOVA, followed by 
Student-Newman-Keuls post hoc testing. Differences were considered significant at $\mathrm{P}<0.05$, with all experimentation conducted at least in triplicate. 


\section{$\underline{\text { Results }}$}

\section{Saracatinib effects on HNSCC proliferation and invasion}

As a first step in our work, we characterized several HNSCC cell lines previously determined to have varying degrees of invasive and metastatic potential (Rothschild et al., 2006; Sano \& Myers, 2007; Yang et al., 2004) for their response to saracatinib. To determine the effect of saracatinib treatment on proliferation in these lines, growth inhibition was assessed by $5 \mathrm{~d}$ 3-(4,5-dimethylthiazol-2-yl)-2,5-diphenyltetrazolium bromide (MTT) assays for cells treated with increasing doses of saracatinib (Fig. 1A). The HN31 and UMSCC1 lines were sensitive to growth inhibition by saracatinib, as indicated by sub-micromolar $I_{50}$ values (Fig. 1A). In contrast, the 1483 line demonstrated a marked resistance to saracatinib, with an $\mathrm{IC}_{50}=7.60 \mu \mathrm{M}$. Corresponding effects on cell cycle progression are also observed, with increasing saracatinib concentrations resulting in enhanced $\mathrm{G}_{1}$ checkpoint arrest in HN31 and UMSCC1 lines determined by FACS analysis (Fig. 1B). Saracatinib treatment did not alter the percentage of cells undergoing $G_{2}-M$ transition for either line. Arrest of $G_{1}$ was not evident in 1483 cells treated with saracatinib concentrations up to $1 \mu \mathrm{M}$, in agreement with resistance of this line in proliferation analysis (Fig. 1A). Erk1/2 activation, a potent driver of mitogenesis, was impaired in HN31 and UMSCC1 cells at concentrations above $0.5 \mu \mathrm{M}$ as determined by Western blotting (Fig. 1C). Similar results were obtained for the invasive HNSCC lines MSK921 and UMSCC19 (data not shown). Erk1/2 activation was elevated in 1483 cells at doses up to $1 \mu \mathrm{M}$ (Fig. 1C), in agreement with the high $\mathrm{IC}_{50}$ value for this line (Fig. 1A). These data indicate that the HNSCC lines used in this study vary in their proliferative response to saracatinib treatment, and can be segregated into sensitive (HN31 and UMSCC1) and resistant (1483) populations.

Next we evaluated the effect of saracatinib on HNSCC invasion in vitro using modified Boyden chamber transwell assays. In the absence of saracatinib, UMSCC1 and HN31 cells displayed an invasive response to serum, averaging 4150 cells/aggregate field and 1719 cells/aggregate field, respectively (Fig. 1D). 1483 cells were weakly invasive, with an average of 243 cells/aggregate field (Fig. 1D). Increased concentrations of saracatinib resulted in dose-dependent inhibition of HNSCC invasion for all tested lines, 
with reduced invasion compared to control levels of $88 \%$ in UMSCC1, $70 \%$ in $\mathrm{HN} 31$, and $78 \%$ in 1483 cells at the highest evaluated dose $(1.0 \mu \mathrm{M})$ (Fig. 1D). These results indicate that saracatinib directly impacts the ability of HNSCC cell lines to invade in an in vitro setting.

\section{Saracatinib inhibits Src activation and substrate phosphorylation in HNSCC cells}

To determine the impact of saracatinib on Src activity and phosphorylation of invadopodia-related Src substrates, dose-dependence experiments were performed on HN31, UMSCC1 and 1483 cells (Fig. 2). Cell lines were treated with increasing doses of saracatinib for 24 hours, lysed and assessed for phosphorylation by immunoblotting using anti-phosphorylation site-specific antibodies to detect Src activation (pY418) and specific Src phosphorylation sites in downstream substrates (pY410 p130 CAS, pY421 cortactin and pY861 FAK). The phosphorylation of Src at Y418 was inhibited by saracatinib in all tested lines at concentrations between 0.5 and $1.0 \mu \mathrm{M}$ (Fig. 2). Phosphorylation of cortactin at tyrosine 421 and FAK at tyrosine 861 was also reduced within the same range of saracatinib concentrations (FAK phosphorylation in 1483 cells could not be evaluated due to the absence of detectable FAK expression in this line). While phosphorylation of tyrosine 410 in p130 CAS was inhibited within this same dose range in UMSCC1 cells, we observed that p130 CAS phosphorylation was consistently inhibited at lower dose ranges (0.01-0.05 $\mu \mathrm{M})$ in HN31 and 1483 cells (Fig. 2). Immunoblotting with antibodies against total p130 CAS, cortactin and FAK indicate that the expression levels of these proteins are somewhat reduced at high dose levels of saracatinib treatment $(0.5-1 \mu \mathrm{M})$, but not at levels accountable for the resultant decrease of tyrosine phosphorylation at the assayed sites $(0.1-0.5 \mu \mathrm{M})$.

Saracatinib inhibits Src activation, invasion and cervical lymph node metastasis in an orthotopic mouse model of oral squamous cell carcinoma

A mouse orthotopic model of tongue squamous cell carcinoma (Myers et al., 2002) that phenotypically mimics human HNSCC (Fig. 3) was utilized to evaluate the effects of saracatinib on HNSCC progression and invasion in an in vivo setting. Athymic mice with UMSCC1 tongue tumors were randomized and treated with either vehicle or daily 
with $25 \mathrm{mg} / \mathrm{kg}$ saracatinib. At the end of treatment (40d), mice from both groups had similar weight $(24 \mathrm{~g})$ and mean tumor volumes $\left(54.1 \mathrm{~mm}^{3} \pm 2.3 \mathrm{~mm}\right.$ for controls; $43.7 \mathrm{~mm}^{3} \pm 4.6 \mathrm{~mm}$ with saracatinib treatment). The modest impact of saracatinib on UMSCC1 tumor growth was not statistically significant. To determine the impact of saracatinib on Src activity, primary tumors were evaluated for Src and cortactin phosphorylation by immunohistochemistry. Active Src (determined by pY416 SFK staining) and pY421 cortactin labeling in UMSCC1 tumors displayed similar patterns compared to human HNSCC (Fig. 3A). Saracatinib treatment reduced the ratios of pY416 SFK and pY421 cortactin compared to control-treated mice (Figure 3A).

Human HNSCC often displays perineural invasion with regional lymph node involvement. Given the invasive nature of UMSCC1 cells (Fig. 1D), we evaluated the impact of saracatinib treatment on loco-regional tissue invasion and cervical lymph node metastasis in treated mice. Soft tissues from the submental space through the tracheoesophageal region were evaluated for perineural invasion and cervical lymph node metastasis by immunostaining for the epithelial marker cytokeratin 14 . Similar to human tumors, extensive perineural invasion and metastasis to the superficial cervical lymph nodes was evident in 5/6 control treated mice (Fig. 3B). Invasion and lymph node metastasis was found in 1/6 saracatinib-treated mice, and the remaining mice displayed a complete absence of cytokeratin-positive cells associated with nerves, sublingual glands, connective tissue or cervical lymph nodes (Fig. 3B). These data demonstrate that the in vivo ability of saracatinib to down-regulate Src activity and cortactin phosphorylation correlates with decreased invasion and local lymph node metastasis.

\section{Saracatinib inhibits invadopodia formation and matrix degradation in UMSCC1 cells}

UMSCC1 cells plated on fluorescently-labeled gelatin formed centrally localized ventral puncta enriched with cortactin, filamentous $(F)$-actin and phosphotyrosine (Fig. 4A, DMSO), three markers that define invadopodia (Bowden et al., 2006). Spontaneous invadopodia formation was observed in $51 \%$ of UMSCC1 cells by confocal microscopy at a given time, corresponding with focalized areas of matrix clearing (FITC-gelatin) 
(Fig. 4B, 0 $\mathrm{MM}$ dosage point). The gelatin matrix underneath UMSCC1 cells typically displayed degradation encompassing $\sim 53 \%$ of the overlying cell area, reflecting the action of invadopodia-associated MMP activity (Fig. 4C).

UMSCC1 cells plated on FITC-coated gelatin were treated with increasing concentrations of saracatinib, and invadopodia formation and matrix degradation was evaluated by confocal microscopy (Fig. 4). The number of cells containing invadopodia was significantly decreased with increasing saracatinib dosage, with $<2 \%$ of cells having formed invadopodia at concentrations at or above $0.5 \mu \mathrm{M}$ (Fig. 4B). Effects on matrix degradation were more pronounced, where increased saracatinib dosage resulted in similar incremental decreases in degradation (Fig. 4C). UMSCC1 cells treated with $1.0 \mu \mathrm{M}$ saracatinib did not contain invadopodia and were incapable of degrading matrix (Fig. 4A). These cells also lacked focal cortactin localization, had diminished phosphotyrosine levels at focal adhesions and contained disorganized Factin puncta on the ventral membrane surface where invadopodia typically form (Fig. $4 \mathrm{~A}$, arrowheads).

\section{Saracatinib inhibits MMP9 secretion from HNSCC cells}

In addition to MT1-MMP, secretion and activation of MMP2 and MMP9 at invadopodia has been reported to be partially responsible for the observed effects on matrix degradation (Linder, 2007). Src activity regulates MMP2 and MMP9 secretion in fibroblasts (Hsia et al., 2003). We therefore determined the effect of saracatinib on MMP secretion. Confocal immunofluorescence microscopy indicated that UMSCC1 cells have MMP9-containing vesicles localized to invadopodia at sites that correspond with gelatin degradation (Fig. 5A), indicating that MMP9 is concentrated in UMSCC1 invadopodia. To evaluate the impact of saracatinib on MMP2 and MMP9 secretion and activity in HNSCC cells, total cell lysates and conditioned media from saracatinibtreated 1483 and UMSCC1 cells were analyzed for the presence of cellular and secreted MMP2 and MMP9 by Western blotting (Fig. 5B). Saracatinib treatment resulted in modest decreases (up to 24\%) in cellular MMP2 levels at concentrations to 1 $\mu \mathrm{M}$, while cellular MMP9 levels demonstrated up to a two-fold increase under the same concentration range. Although secreted MMP2 was not detected in the media of either 
cell line, both lines secreted detectible amounts of MMP9 (Fig. 5B). Treatment of either line with saracatinib inhibited MMP9 secretion, and each line displayed differential drug sensitivity. Detectible MMP9 secretion from 1483 cells was largely absent at the lowest evaluated concentration $(0.01 \mu \mathrm{M})$ whereas secretion from the more invasive UMSCC1 line was inhibited at concentrations of $0.5 \mu \mathrm{M}$ and above (Fig. 5B). The secreted MMP9 from both lines displayed proteolytic activity when assayed by gelatin zymography (Fig. 5C). Saracatinib concentrations up to $0.1 \mu \mathrm{M}$ did not significantly affect MMP9 activity as determined by ANOVA analysis for both lines, although mean values for 1483 cells treated with these lower doses were consistently below control levels (Fig. 5C). Higher saracatinib concentrations $(0.5 \mu \mathrm{M}$ and $1.0 \mu \mathrm{M})$ reduced MMP9 activity to respective mean values of $39 \%$ and $25 \%$ for control levels in 1483 cells, and $22 \%$ and $12 \%$ in UMSCC1 cells (Fig. 5B,C). These data indicate that saracatinib treatment of HNSCC cells leads to selective inhibition of MMP9 secretion in 1483 and UMSCC1 cells, preventing efficient enzymatic degradation of extracellular matrix components. 


\section{Discussion}

The present study demonstrates that inhibition of HNSCC invasion in preclinical in vitro and in vivo settings by saracatinib directly corresponds to disruption of HNSCC invadopodia formation and function, identifying invadopodia as a potential downstream target of therapeutic Src kinase inhibition in HNSCC and other invasive human cancers. Invasive HNSCC presents a difficult problem in patient care, given the proximity of most tumors to multiple vital organ sites in the head and neck region. Disregulation of signaling pathways that promote and sustain invasion impinge on adhesion- and cytoskeletal-associated proteins. These proteins function in concert with MMPs to enable tumor cells to degrade and protrude through an encapsulating ECM, allowing movement into neighboring tissues. The ability of saracatinib to ablate invadopodia and the associated invasive behavior of HNSCC cells in mice provides further evidence for a direct link between invadopodia activity and tumor invasion, shedding light on the specific invasion-promoting cellular processes perturbed by Src kinase inhibition.

The HNSCC lines used in this study displayed differential responses to saracatinib in terms of anti-proliferate effects, with some lines (HN31 and UMSCC1) having submicromolar sensitivity, cell cycle inhibition and decreased ERK1/2 activity and others (1483) demonstrating resistance to the drug at concentrations up to $1 \mu \mathrm{M}$ (Fig. 1A-C). While a recent study reported $\mathrm{IC}_{50}$ saracatinib values near $1 \mu \mathrm{M}$ for five different HNSCC lines (Koppikar et al., 2008), our findings are in line with the wider range of $I_{50}$ values reported for different HNSCC lines treated with the non-related Src kinase inhibitor dasatinib (Johnson et al., 2005) as well as in other tumor cell types (Boyer et al., 2002; Johnson et al., 2005; Jones et al., 2002). These reports taken together with our data suggest that the HNSCC lines utilized in this study fall within the typical in vitro proliferative response profile to therapeutic Src inhibition. While the underlying compensatory mechanism for saracatinib resistance in 1483 cells is unknown, future expression profiling of resistant and sensitive lines may provide insight into the molecular nature of saracatinib resistance, as has been recently conducted for the EGFR inhibitor gefitinib in a variety of HNSCC lines (Frederick et al., 2007). 
Saracatinib treatment resulted in in vitro anti-invasive activity, impaired Src activation and tyrosine phosphorylation of FAK and p130 CAS in all analyzed HNSCC lines (Fig. 1D; Fig. 2). This is in accord with other studies on other HNSCC lines utilizing saracatinib or dasatinib as single agents (Johnson et al., 2005; Koppikar et al., 2008; Nozawa et al., 2008). Tyrosine phosphorylation of FAK and p130 CAS have been commonly utilized as downstream indicators for preclinical therapeutic anti-Src efficacy, since Src-mediated phosphorylation of these proteins are critical events in enabling tumor invasiveness (Brabek et al., 2005; Zhao \& Guan, 2009). In addition, we show that cortactin tyrosine phosphorylation was also reduced following saracatinib treatment (Fig. 2). Cortactin is a Src substrate commonly overexpressed in invasive HNSCC and regulates invadopodia formation (Rodrigo et al., 2000; Rothschild et al., 2006; Weaver, 2008). Cortactin phosphorylation is important for tumor cell motility and matrix degradation at invadopodia (Ayala et al., 2008; Oser et al., 2009; Rothschild et al., 2006). These results suggest that cortactin tyrosine phosphorylation status can serve as an additional downstream monitor of Src activity and invasive potential in HNSCC cells where Src kinase function is impaired.

While saracatinib was able to modestly decrease the size of in vivo UMSCC1 tongue tumors, the reduction in tumor size was not significant compared to untreated controls. UMSCC1 cell growth is inhibited by saracatinib in vitro (Fig. 1A), implying that microenvironmental factors such as inflammatory cytokines, growth factors, neoangiogenic and hypoxic aspects responsible for promoting and maintaining HNSCC growth (Pries \& Wollenberg, 2006; Timar et al., 2005) may partially circumvent the growth-inhibitory effects of saracatinib in UMSCC1 xenografts. On the other hand, saracatinib displays in vivo anti-invasive properties by potently inhibiting perineural invasion and cervical lymph node metastasis. Suppressed Src activation and cortactin tyrosine phosphorylation in primary tumors (Fig. 4A) supports this conclusion, as signaling through these proteins promotes invasion and metastatic spread (Weaver, 2008; Yeatman, 2004). Recent findings in a comparable xenograft system utilizing mice treated with dasatinib demonstrated reduced Src and FAK activity (Sen et al., 2009). EGFR overexpression is common in HNSCC, resulting in enhanced Src activity, cortactin phosphorylation and tumor invasiveness (Koppikar et al., 2008; Rothschild et 
al., 2006). Clinical EGFR inhibitors also display anti-invasive activity and impair invadopodia formation in preclinical settings (Huang et al., 2002; Yamaguchi et al., 2005; Yang et al., 2004), suggesting in light of our findings that inhibiting EGFR activity in HNSCC impairs Src activation and substrate phosphorylation required for invasion. This is supported by emerging rationale for dual targeting of EGFR and Src in treating advanced HNSCC (Egloff \& Grandis, 2008).

Elevated Src activity is necessary and essential for invadopodia formation (Chen et al., 1985; Chen et al., 1984). The ability of saracatinib to ablate invadopodia and associated matrix degradation demonstrates that a clinically utilized Src inhibitor disrupts a vital subcellular structure required for tumor invasion. Src-induced invadopodia formation in carcinoma cells first targets cortactin and F-actin formation at matrix adhesion sites, forming a core preinvadopodia complex. Recruitment of MT1MMP to preinvadopodia initiates matrix degradation and invadopodia maturation, with further maturation involving dissolution of the cortactin-F-actin complex, focal retention of MT1-MMP and continued proteolytic activity (Artym et al., 2006). While rudimentary invadopodia-like F-actin structures formed in UMSCC1 cells treated with inhibitory concentrations of saracatinib, they do not contain cortactin (Fig. 4A), which is essential for invadopodia formation (Artym et al., 2006), indicating that Src kinase activity is required for cortactin localization to invadopodia. Similar results have been shown through the use of kinase-inactive Src constructs (Bowden et al., 2006). Tyrosine phosphorylation of invadopodia proteins is strongly linked with the ability to degrade extracellular matrix (Bowden et al., 2006), with Src phosphorylation of cortactin (Ayala et al., 2008), paxillin (Badowski et al., 2008), and ASAP1 (Bharti et al., 2007) requisite for invadopodia formation and/or proteolytic activity. Src kinase inhibition therefore displays at least a two-fold effect on substrates in invadopodia by impairing proper preinvadopodia targeting and perturbing phosphotyrosine-based signaling dynamics involved in regulating invadopodia maturation and function. Tyrosine phosphorylation in peripheral focal adhesions was observed in UMSCC1 cells, indicating saracatinib may also perturb focal adhesion formation and/or function given the critical role for Src and related kinases in these structures (Frame, 2004). 
MMP activity is essential for HNSCC invadopodia formation and function (Clark et al., 2007). The impairment of MMP9 secretion and activation from HNSCC cells treated with saracatinib indicates that Src kinase activity is required for targeting and secretion of MMP9-containing vesicles at invadopodia. This is in agreement with observed effects of Src kinase inhibition on MMP9 secretion in other tumor types (CortesReynosa et al., 2008; Lee et al., 2005). The lack of apparent MMP2 secretion in the HNSCC lines used in our studies precluded evaluation of this metalloproteinase, and is likely characteristic to these lines since other HNSCC cells secrete MMP2 (Clark et al., 2007). Localization of transmembrane and secreted MMPs to invadopodia involves directed trafficking of vesicles emanating from the trans-Golgi network, where a dynamin-2-N-WASp-Arp2/3-cortactin complex has been implicated in coupling cortical actin regulation with invadopodia membrane dynamics (Buccione et al., 2004). Cortactin has been implicated as a key regulator of MT1-MMP surface expression and MMP2 and MMP9 secretion in HNSCC (Clark \& Weaver, 2008; Clark et al., 2007). While it is currently unknown how cortactin regulates the targeting of MMP-containing vesicles, Src-mediated phosphorylation may play a vital role since tyrosine phosphorylation of cortactin increases its binding to vesicle-associated proteins (Ammer \& Weed, 2008) and is required for efficient invadopodia-mediated ECM degradation (Ayala et al., 2008; Webb et al., 2007).

Our results indicate that disruption of Src activity by saracatinib impairs HNSCC cell invasion and lymph node metastasis by preventing invadopodia formation and function, identifying a cellular mechanism that may be universally impacted by Src inhibition in invasive carcinoma cells. In addition to Src and related kinases, saracatinib also inhibits Abl kinase, an activity that has been exploited to evaluate imatinib-resistant chronic myelogenous leukemia (CML) cases expressing the constitutively active BCR-Abl Philadelphia chromosome gene product (Gwanmesia et al., 2009). Along with the tumor-promoting activities in CML, elevated Abl kinase activity has been shown to be important in breast (Srinivasan \& Plattner, 2006) and non small cell lung cancer (Lin et al., 2007), indicating Abl kinase activity has a functional role in solid tumor progression (Lin \& Arlinghaus, 2008). Abl expression has been evaluated in oral squamous cell carcinoma and correlates with tumor stage (Yanagawa et al., 2000), suggesting that 
elevated Abl expression may contribute to HNSCC progression. While a mechanism pertaining to Abl function in solid tumor invasion or invadopodia function has not been reported, Abl does bind and phosphorylate cortactin, (Boyle et al., 2007), raising the potential for Abl kinase to play a role in cortactin-based invadopodia function in HNSCC and other invasive Abl-expressing solid tumors. Such a role for Abl in regulating tumor invasion would also likely be impaired by saracatinib and other dual Src/Abl inhibitory compounds.

In addition to carcinoma invadopodia, Src-mediated processes in non-cancerous cell types involved in promoting invasion may also be impacted by Src family kinase inhibition. The motility and protease remodeling ability of tumor stromal fibroblasts involved in enabling collective HNSCC invasion could be affected by impairing Src family kinases in vivo (Gaggioli et al., 2007), as well as the proinvasive properties of tumor-associated macrophages (Condeelis \& Pollard, 2006). The ability of saracatinib and other Src inhibitors to impair functions of different cellular types that propagate tumor invasion provides the opportunity for the future discovery of additional Src-based cellular mechanisms utilized during tumor progression. 


\section{Acknowledgements}

We thank J. Myers and M. Younes (Department of Head and Neck Surgery, University of Texas M.D. Anderson Cancer Center, Houston, TX) for UMSCC1 cells and related help, P. Turner and K. Secrest of the West Virginia University Department of Pathology Tissue Bank for histochemistry services. The contributions of the West Virginia University Microscopic Imaging Facility, Mary Babb Randolph Cancer Center are gratefully acknowledged. This work was supported by a grant from AstraZeneca to DR, BF and SAW and by NIH grants R01 DE014578 and P20 RR16440 to SAW. LCK was supported in part by the West Virginia University Office of Research and Graduate Education. 


\section{References}

Ammer, A.G. \& Weed, S.A. (2008). Cortactin branches out: roles in regulating protrusive actin dynamics. Cell Motil Cytoskeleton, 65, 687-707.

Artym, V.V., Zhang, Y., Seillier-Moiseiwitsch, F., Yamada, K.M. \& Mueller, S.C. (2006). Dynamic interactions of cortactin and membrane type 1 matrix metalloproteinase at invadopodia: defining the stages of invadopodia formation and function. Cancer Res, 66, 3034-43.

Ayala, I., Baldassarre, M., Giacchetti, G., Caldieri, G., Tete, S., Luini, A. \& Buccione, R. (2008). Multiple regulatory inputs converge on cortactin to control invadopodia biogenesis and extracellular matrix degradation. J Cell Sci.

Badowski, C., Pawlak, G., Grichine, A., Chabadel, A., Oddou, C., Jurdic, P., Pfaff, M., Albiges-Rizo, C. \& Block, M.R. (2008). Paxillin phosphorylation controls Invadopodia/podosomes spatiotemporal organization. Mol Biol Cell, 19, 633-45.

Bharti, S., Inoue, H., Bharti, K., Hirsch, D.S., Nie, Z., Yoon, H.Y., Artym, V., Yamada, K.M., Mueller, S.C., Barr, V.A. \& Randazzo, P.A. (2007). Src-dependent phosphorylation of ASAP1 regulates podosomes. Mol Cell Biol, 27, 8271-83.

Bowden, E.T., Onikoyi, E., Slack, R., Myoui, A., Yoneda, T., Yamada, K.M. \& Mueller, S.C. (2006). Co-localization of cortactin and phosphotyrosine identifies active invadopodia in human breast cancer cells. Exp Cell Res, 312, 1240-53.

Boyer, B., Bourgeois, Y. \& Poupon, M.F. (2002). Src kinase contributes to the metastatic spread of carcinoma cells. Oncogene, 21, 2347-56.

Boyle, S.N., Michaud, G.A., Schweitzer, B., Predki, P.F. \& Koleske, A.J. (2007). A critical role for cortactin phosphorylation by Abl-family kinases in PDGF-induced dorsal-wave formation. Curr Biol, 17, 445-51.

Brabek, J., Constancio, S.S., Siesser, P.F., Shin, N.Y., Pozzi, A. \& Hanks, S.K. (2005). Crk-associated substrate tyrosine phosphorylation sites are critical for invasion and metastasis of SRC-transformed cells. Mol Cancer Res, 3, 307-15.

Buccione, R., Orth, J.D. \& McNiven, M.A. (2004). Foot and mouth: podosomes, invadopodia and circular dorsal ruffles. Nat Rev Mol Cell Biol, 5, 647-57. 
Chen, W.T., Chen, J.M., Parsons, S.J. \& Parsons, J.T. (1985). Local degradation of fibronectin at sites of expression of the transforming gene product pp60src. Nature, 316, 156-8.

Chen, W.T., Olden, K., Bernard, B.A. \& Chu, F.F. (1984). Expression of transformationassociated protease(s) that degrade fibronectin at cell contact sites. J Cell Biol, 98, 1546-55.

Clark, E.S. \& Weaver, A.M. (2008). A new role for cortactin in invadopodia: Regulation of protease secretion. Eur J Cell Biol,

Clark, E.S., Whigham, A.S., Yarbrough, W.G. \& Weaver, A.M. (2007). Cortactin is an essential regulator of matrix metalloproteinase secretion and extracellular matrix degradation in invadopodia. Cancer Res, 67, 4227-35.

Condeelis, J. \& Pollard, J.W. (2006). Macrophages: obligate partners for tumor cell migration, invasion, and metastasis. Cell, 124, 263-6.

Cortes-Reynosa, P., Robledo, T., Macias-Silva, M., Wu, S.V. \& Salazar, E.P. (2008). Src kinase regulates metalloproteinase- 9 secretion induced by type IV collagen in MCF-7 human breast cancer cells. Matrix Biol, 27, 220-31.

Dolgin, E. (2009). Cancer metastasis scrutinized. Nature, 461, 854-5.

Egloff, A.M. \& Grandis, J.R. (2008). Targeting epidermal growth factor receptor and SRC pathways in head and neck cancer. Semin Oncol, 35, 286-97.

Frame, M.C. (2004). Newest findings on the oldest oncogene; how activated src does it. J Cell Sci, 117, 989-98.

Frederick, B.A., Helfrich, B.A., Coldren, C.D., Zheng, D., Chan, D., Bunn, P.A., Jr. \& Raben, D. (2007). Epithelial to mesenchymal transition predicts gefitinib resistance in cell lines of head and neck squamous cell carcinoma and non-small cell lung carcinoma. Mol Cancer Ther, 6, 1683-91.

Gaggioli, C., Hooper, S., Hidalgo-Carcedo, C., Grosse, R., Marshall, J.F., Harrington, K. \& Sahai, E. (2007). Fibroblast-led collective invasion of carcinoma cells with differing roles for RhoGTPases in leading and following cells. Nat Cell Biol, 9, 1392-400. 
Gimona, M., Buccione, R., Courtneidge, S.A. \& Linder, S. (2008). Assembly and biological role of podosomes and invadopodia. Curr Opin Cell Biol.

Green, T.P., Fennell, M., Whittaker, R., Curwen, J., Jacobs, V., Allen, J., Logie, A., Hargreaves, J., Hickinson, D.M., Wilkinson, R.W., Elvin, P., Boyer, B., Carragher, N., Ple, P.A., Bermingham, A., Holdgate, G.A., Ward, W.H., Hennequin, L.F., Davies, B.R. \& Costello, G.F. (2009). Preclinical anticancer activity of the potent, oral Src inhibitor AZD0530. Mol Oncol.

Gwanmesia, P.M., Romanski, A., Schwarz, K., Bacic, B., Ruthardt, M. \& Ottmann, O.G. (2009). The effect of the dual Src/Abl kinase inhibitor AZD0530 on Philadelphia positive leukaemia cell lines. BMC Cancer, 9, 53.

Hennequin, L.F., Allen, J., Breed, J., Curwen, J., Fennell, M., Green, T.P., Lambert-van der Brempt, C., Morgentin, R., Norman, R.A., Olivier, A., Otterbein, L., Ple, P.A., Warin, N. \& Costello, G. (2006). N-(5-chloro-1,3-benzodioxol-4-yl)-7-[2-(4methylpiperazin-1-yl)ethoxy]-5- (tetrahydro-2H-pyran-4-yloxy)quinazolin-4-amine, a novel, highly selective, orally available, dual-specific c-Src/Abl kinase inhibitor. J Med Chem, 49, 6465-88.

Herynk, M.H., Beyer, A.R., Cui, Y., Weiss, H., Anderson, E., Green, T.P. \& Fuqua, S.A. (2006). Cooperative action of tamoxifen and c-Src inhibition in preventing the growth of estrogen receptor-positive human breast cancer cells. Mol Cancer Ther, 5, 3023-31.

Hsia, D.A., Mitra, S.K., Hauck, C.R., Streblow, D.N., Nelson, J.A., llic, D., Huang, S., Li, E., Nemerow, G.R., Leng, J., Spencer, K.S., Cheresh, D.A. \& Schlaepfer, D.D. (2003). Differential regulation of cell motility and invasion by FAK. J Cell Biol, $160,753-67$.

Huang, S.M., Li, J. \& Harari, P.M. (2002). Molecular inhibition of angiogenesis and metastatic potential in human squamous cell carcinomas after epidermal growth factor receptor blockade. Mol Cancer Ther, 1, 507-14.

Irby, R.B. \& Yeatman, T.J. (2000). Role of Src expression and activation in human cancer. Oncogene, 19, 5636-42.

Johnson, F.M., Saigal, B., Talpaz, M. \& Donato, N.J. (2005). Dasatinib (BMS-354825) tyrosine kinase inhibitor suppresses invasion and induces cell cycle arrest and apoptosis of head and neck squamous cell carcinoma and non-small cell lung cancer cells. Clin Cancer Res, 11, 6924-32. 
Jones, R.J., Avizienyte, E., Wyke, A.W., Owens, D.W., Brunton, V.G. \& Frame, M.C. (2002). Elevated $c$-Src is linked to altered cell-matrix adhesion rather than proliferation in KM12C human colorectal cancer cells. Br J Cancer, 87, 1128-35.

Kelley, L.C., Shahab, S. \& Weed, S.A. (2008). Actin cytoskeletal mediators of motility and invasion amplified and overexpressed in head and neck cancer. Clin Exp Metastasis.

Kopetz, S., Shah, A.N. \& Gallick, G.E. (2007). Src continues aging: current and future clinical directions. Clin Cancer Res, 13, 7232-6.

Koppikar, P., Choi, S.H., Egloff, A.M., Cai, Q., Suzuki, S., Freilino, M., Nozawa, H., Thomas, S.M., Gooding, W.E., Siegfried, J.M. \& Grandis, J.R. (2008). Combined inhibition of c-Src and epidermal growth factor receptor abrogates growth and invasion of head and neck squamous cell carcinoma. Clin Cancer Res, 14, 428491.

Kramer, R.H., Shen, X. \& Zhou, H. (2005). Tumor cell invasion and survival in head and neck cancer. Cancer Metastasis Rev, 24, 35-45.

Lee, J.C., Maa, M.C., Yu, H.S., Wang, J.H., Yen, C.K., Wang, S.T., Chen, Y.J., Liu, Y., Jin, Y.T. \& Leu, T.H. (2005). Butyrate regulates the expression of C-Src and focal adhesion kinase and inhibits cell invasion of human colon cancer cells. Mol Carcinog, 43, 207-14.

Lin, J. \& Arlinghaus, R. (2008). Activated c-Abl tyrosine kinase in malignant solid tumors. Oncogene, 27, 4385-91.

Lin, J., Sun, T., Ji, L., Deng, W., Roth, J., Minna, J. \& Arlinghaus, R. (2007). Oncogenic activation of c-Abl in non-small cell lung cancer cells lacking FUS1 expression: inhibition of c-Abl by the tumor suppressor gene product Fus1. Oncogene, 26, 6989-96.

Linder, S. (2007). The matrix corroded: podosomes and invadopodia in extracellular matrix degradation. Trends Cell Biol, 17, 107-17.

Mueller, S.C., Yeh, Y. \& Chen, W.T. (1992). Tyrosine phosphorylation of membrane proteins mediates cellular invasion by transformed cells. J Cell Biol, 119, 130925. 
Myers, J.N., Holsinger, F.C., Jasser, S.A., Bekele, B.N. \& Fidler, I.J. (2002). An orthotopic nude mouse model of oral tongue squamous cell carcinoma. Clin Cancer Res, 8, 293-8.

Nozawa, H., Howell, G., Suzuki, S., Zhang, Q., Qi, Y., Klein-Seetharaman, J., Wells, A., Grandis, J.R. \& Thomas, S.M. (2008). Combined inhibition of PLC\{gamma\}-1 and c-Src abrogates epidermal growth factor receptor-mediated head and neck squamous cell carcinoma invasion. Clin Cancer Res, 14, 4336-44.

Oser, M., Yamaguchi, H., Mader, C.C., Bravo-Cordero, J.J., Arias, M., Chen, X., Desmarais, V., van Rheenen, J., Koleske, A.J. \& Condeelis, J. (2009). Cortactin regulates cofilin and N-WASp activities to control the stages of invadopodium assembly and maturation. $J$ Cell Biol, 186, 571-87.

Park, S.I., Zhang, J., Phillips, K.A., Araujo, J.C., Najjar, A.M., Volgin, A.Y., Gelovani, J.G., Kim, S.J., Wang, Z. \& Gallick, G.E. (2008). Targeting SRC family kinases inhibits growth and lymph node metastases of prostate cancer in an orthotopic nude mouse model. Cancer Res, 68, 3323-33.

Pries, R. \& Wollenberg, B. (2006). Cytokines in head and neck cancer. Cytokine Growth Factor Rev, 17, 141-6.

Rodrigo, J.P., Garcia, L.A., Ramos, S., Lazo, P.S. \& Suarez, C. (2000). EMS1 gene amplification correlates with poor prognosis in squamous cell carcinomas of the head and neck. Clin Cancer Res, 6, 3177-82.

Rosenthal, E.L. \& Matrisian, L.M. (2006). Matrix metalloproteases in head and neck cancer. Head Neck, 28, 639-48.

Rothschild, B.L., Shim, A.H., Ammer, A.G., Kelley, L.C., Irby, K.B., Head, J.A., Chen, L., Varella-Garcia, M., Sacks, P.G., Frederick, B., Raben, D. \& Weed, S.A. (2006). Cortactin overexpression regulates actin-related protein $2 / 3$ complex activity, motility, and invasion in carcinomas with chromosome 11 q13 amplification. Cancer Res, 66, 8017-25.

Sano, D. \& Myers, J.N. (2007). Metastasis of squamous cell carcinoma of the oral tongue. Cancer Metastasis Rev, 26, 645-62.

Sen, B., Saigal, B., Parikh, N., Gallick, G. \& Johnson, F.M. (2009). Sustained Src inhibition results in signal transducer and activator of transcription 3 (STAT3) 
activation and cancer cell survival via altered Janus-activated kinase-STAT3 binding. Cancer Res, 69, 1958-65.

Srinivasan, D. \& Plattner, R. (2006). Activation of Abl tyrosine kinases promotes invasion of aggressive breast cancer cells. Cancer Res, 66, 5648-55.

Summy, J.M. \& Gallick, G.E. (2003). Src family kinases in tumor progression and metastasis. Cancer Metastasis Rev, 22, 337-58.

Summy, J.M. \& Gallick, G.E. (2006). Treatment for advanced tumors: SRC reclaims center stage. Clin Cancer Res, 12, 1398-401.

Timar, J., Csuka, O., Remenar, E., Repassy, G. \& Kasler, M. (2005). Progression of head and neck squamous cell cancer. Cancer Metastasis Rev, 24, 107-27.

van Oijen, M.G., Rijksen, G., ten Broek, F.W. \& Slootweg, P.J. (1998). Overexpression of $\mathrm{c}$-Src in areas of hyperproliferation in head and neck cancer, premalignant lesions and benign mucosal disorders. J Oral Pathol Med, 27, 147-52.

Weaver, A.M. (2006). Invadopodia: specialized cell structures for cancer invasion. Clin Exp Metastasis, 23, 97-105.

Weaver, A.M. (2008). Cortactin in tumor invasiveness. Cancer Lett, 265, 157-66.

Webb, B.A., Jia, L., Eves, R. \& Mak, A.S. (2007). Dissecting the functional domain requirements of cortactin in invadopodia formation. Eur J Cell Biol, 86, 189-206.

Yamaguchi, H., Lorenz, M., Kempiak, S., Sarmiento, C., Coniglio, S., Symons, M., Segall, J., Eddy, R., Miki, H., Takenawa, T. \& Condeelis, J. (2005). Molecular mechanisms of invadopodium formation: the role of the N-WASP-Arp2/3 complex pathway and cofilin. J Cell Biol, 168, 441-52.

Yanagawa, T., Harada, H., Iwasa, S., Tabuchi, K., Omura, K., Suzuki, H., Yusa, H., Yamagata, K., Onizawa, K., Ishii, T. \& Yoshida, H. (2000). c-Abl expression in oral squamous cell carcinomas. Oral Oncol, 36, 89-94.

Yang, Z., Bagheri-Yarmand, R., Wang, R.A., Adam, L., Papadimitrakopoulou, V.V., Clayman, G.L., El-Naggar, A., Lotan, R., Barnes, C.J., Hong, W.K. \& Kumar, R. (2004). The epidermal growth factor receptor tyrosine kinase inhibitor ZD1839 
(Iressa) suppresses c-Src and Pak1 pathways and invasiveness of human cancer cells. Clin Cancer Res, 10, 658-67.

Yeatman, T.J. (2004). A renaissance for SRC. Nat Rev Cancer, 4, 470-80.

Zhang, Q., Thomas, S.M., Xi, S., Smithgall, T.E., Siegfried, J.M., Kamens, J., Gooding, W.E. \& Grandis, J.R. (2004). SRC family kinases mediate epidermal growth factor receptor ligand cleavage, proliferation, and invasion of head and neck cancer cells. Cancer Res, 64, 6166-73.

Zhao, J. \& Guan, J.L. (2009). Signal transduction by focal adhesion kinase in cancer. Cancer Metastasis Rev, 28, 35-49. 


\section{Figure Legends}

Figure 1 Effects of saracatinib on HNSCC proliferation, cell cycle progression, Erk1/2 activation and in vitro invasion. A. $\mathrm{IC}_{50}$ values for cell growth determined by 5 day MTT assays for the indicated HNSCC lines treated with 0-10 $\mu \mathrm{M}$ saracatinib. Mean values are shown for each line from three independent assays. B. Impact of saracatinib on HNSCC cell cycle progression. HNSCC cell lines were treated with the indicated amounts of saracatinib for 24 hours, fixed labeled with propidium iodide to assess DNA content, and analyzed for cell cycle status by fluorescence-activated cell sorting. Results show the average percentage of cells in each cell cycle phase as indicated on the left. Bars, SD of two independent experiments. C. Effects of saracatinib on Erk1/2 activity. HNSCC cells were treated with saracatinib for 24 hours at the indicated doses, lysed and analyzed by Western blotting with phosphorylation-specific ( $\mathrm{EErk1/2)}$ and total Erk1/2 antibodies. Blots shown are representative of three different experiments, with indicated band intensities shown relative to no treatment $(0 \mu \mathrm{M})$ for each cell line. $\mathbf{D}$. Saracatinib inhibits in vitro HNSCC invasion. HNSCC cells $\left(1 \times 10^{5}\right)$ were plated in Matrigel-coated transwells alone or with increasing concentrations of saracatinib. After $2 \mathrm{~h}$, invasion was stimulated with 5\% FBS and cells were allowed to invade for $12 \mathrm{~h}$ (UMSCC1) or $24 \mathrm{~h}$ (HN31 and 1483). Invaded cells were quantified by brightfield microscopy. Bars, SEM of three independent experiments.

Figure 2 Saracatinib inhibits Src activity and downstream Src substrate phosphorylation in HNSCC cell lines. HN31, UMSCC1 and 1483 cells were treated with DMSO vehicle or saracatinib $(0.01-1 \mu \mathrm{M})$ for $24 \mathrm{~h}$. Cells were lysed and total protein amounts were analyzed by Western blotting with total or phosphorylation site-specific antibodies for Src and the indicated substrates. Blots shown are representative of at least four independent experiments, with band intensities for each substrate quantified relative to the untreated $(0 \mu \mathrm{M})$ condition for each cell line.

Figure 3 Saracatinib inhibits Src activity, perineural invasion and cervical lymph node metastasis in orthotopic UMSCC1 tongue tumors. A. UMSCC1 tongue tumors from representative control-treated or saracatinib-treated mice were sectioned and stained with hematoxylin and eosin (H\&E) or by $\mathrm{IHC}$ with the indicated antibodies (left). A case 
of human HNSCC was evaluated in parallel as a positive control. The pY416 SFK and pY421 cortactin ratios from saracatinib treated to control levels are indicated. Bars, 100 $\mu \mathrm{m}$. B. Locoregional invasion and lymph node metastasis is inhibited by saracatinib. Submental and associated tracheoesophageal tissue from control treated and saracatinib treated mice was immunostained for cytokeratin 14 to detect cells of epithelial origin. Magnified regions containing a single sublingual nerve and superficial cervical lymph node are shown for clarity. Inset shows a magnified cortical region of superficial cervical lymph nodes from control and saracatinib treated mice. Arrowheads denote metastasized UMSCC1 cells. N; sublingual nerve, ED; excretory duct. Bars 100 $\mu \mathrm{m}$; inset, $50 \mu \mathrm{m}$.

Figure 4 Saracatinib inhibits invadopodia formation and ECM degradation. A. Representative images of UMSCC1 cells treated with different saracatinib concentrations. UMSCC1 cells plated on FITC-gelatin coated coverslips (pseudocolored white) for $2 \mathrm{~h}$ were treated with saracatinib as indicated (left) for $6 \mathrm{~h}$. Cells were labeled to visualize F-actin (red), cortactin (green) and phosphotyrosine (blue). Arrows denote invadopodia and corresponding colocalized areas of focal matrix degradation with invadopodia components. Treatment with $1.0 \mu \mathrm{M}$ saracatinib resulted in F-actin aggregates lacking cortactin but accumulated at cytoplasmic sites where invadopodia typically occur (arrowheads). Bar, $10 \mu \mathrm{m}$. B. UMSCC1 cells treated with increasing concentrations of saracatinib were stained as in $\mathbf{A}$ and quantified to determine the percentage of cells that produced functional invadopodia, presented as the mean \pm SEM. All treatment groups were significantly different from each another based on a one-way ANOVA $(p<0.05)$ except 0 and $0.01 \mu \mathrm{M}$, and 0.5 and $1.0 \mu \mathrm{M}$ pairs. C. Saracatinib decreases the ability of UNSCC1 cells to degrade ECM. The percentage of gelatin degradation per cell area for the cell population analyzed in $B$ is shown with the mean \pm SEM.

Figure 5 MMP9 secretion and ECM degradation activity in HNSCC cells is blocked by saracatinib. A. Localization of MMP9-containing vesicles in UMSCC1 invadopodia. Top: UMSCC1 cells plated on FITC-coated gelatin coverslips for $2 \mathrm{~h}$ were fixed and labeled with antibodies against cortactin and MMP9. The merged image indicates 
areas of cortactin and MMP9 co-localization (yellow; white arrows) that correspond with sites of focal gelatin degradation (black arrows). Bar; $10 \mu \mathrm{m}$, Asterisk; regions of global matrix degradation due to secreted protease activity. Bottom: Magnified view of indicated Top region. B. Inhibition of MMP9 secretion by saracatinib. Total cell lysates (cell) and aliquots of normalized conditioned media containing secreted MMPs (sec) from 1483 and UMSCC1 cells treated with increasing doses of saracatinib (bottom) were assayed for the presence of MMP2 and MMP9 by immunoblotting. Band intensities relative to control $(0 \mu \mathrm{M})$ are shown for each treatment condition; secreted MMP2 was not detected and therefore not quantified. C. Gelatin zymography of MMP9 activity. Representative zymograms from conditioned media of 1483 or UMSCC1 cells cultured with the indicated saracatinib concentrations (bottom). DMEM was used as a negative control (M), DMEM containing 10\% FBS (FBS) was used as a positive control for zymography. Graphs, densitometric analysis of MMP9 zymography. Percentage of MMP9 gelatin clearing is represented and the mean \pm SEM for each cell line from three independent experiments. 
A

$\mathrm{IC}_{50}$ Values for

HNSCC Lines

HN31 $0.74 \mu \mathrm{M}$

UMSCC1 $0.46 \mu \mathrm{M}$

$1483 \quad 7.60 \mu \mathrm{M}$
B

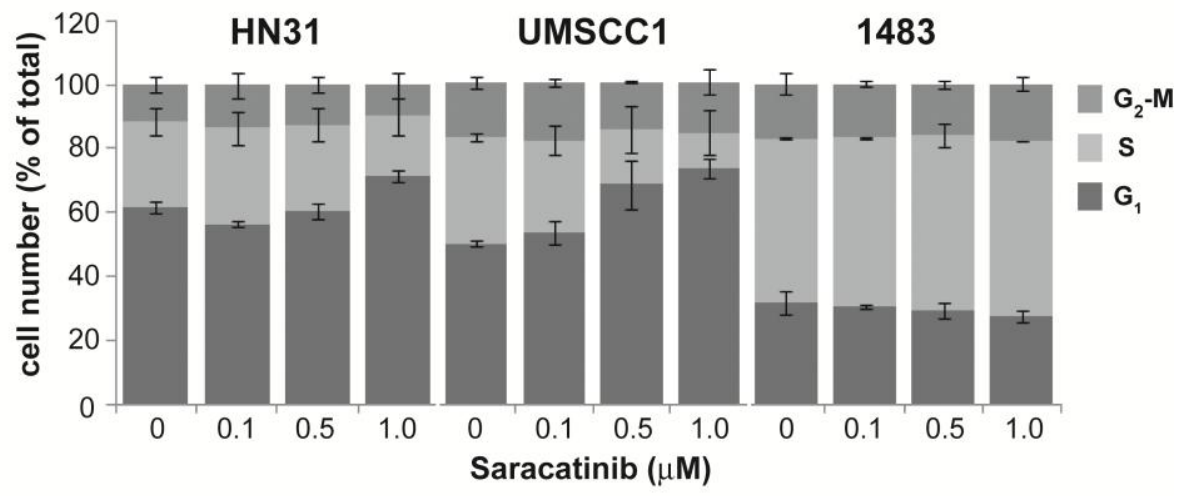

C

$\begin{array}{lll}\text { HN31 UMSCC1 } & 1483\end{array}$

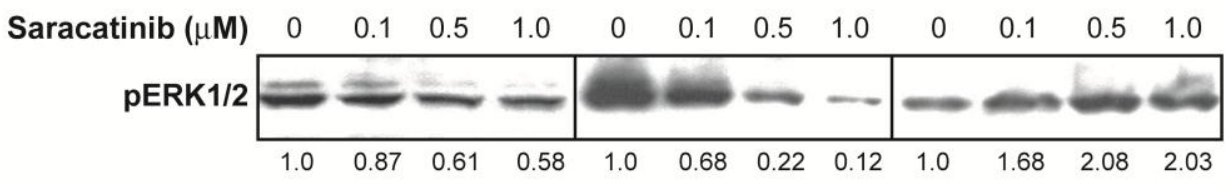

ERK1/2

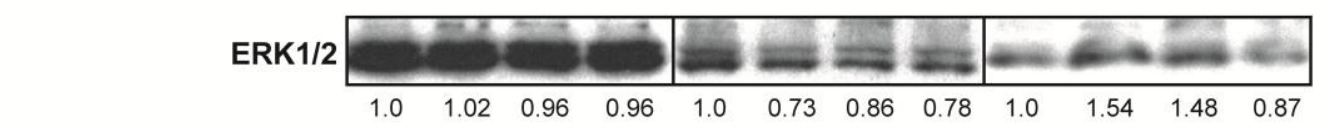

D

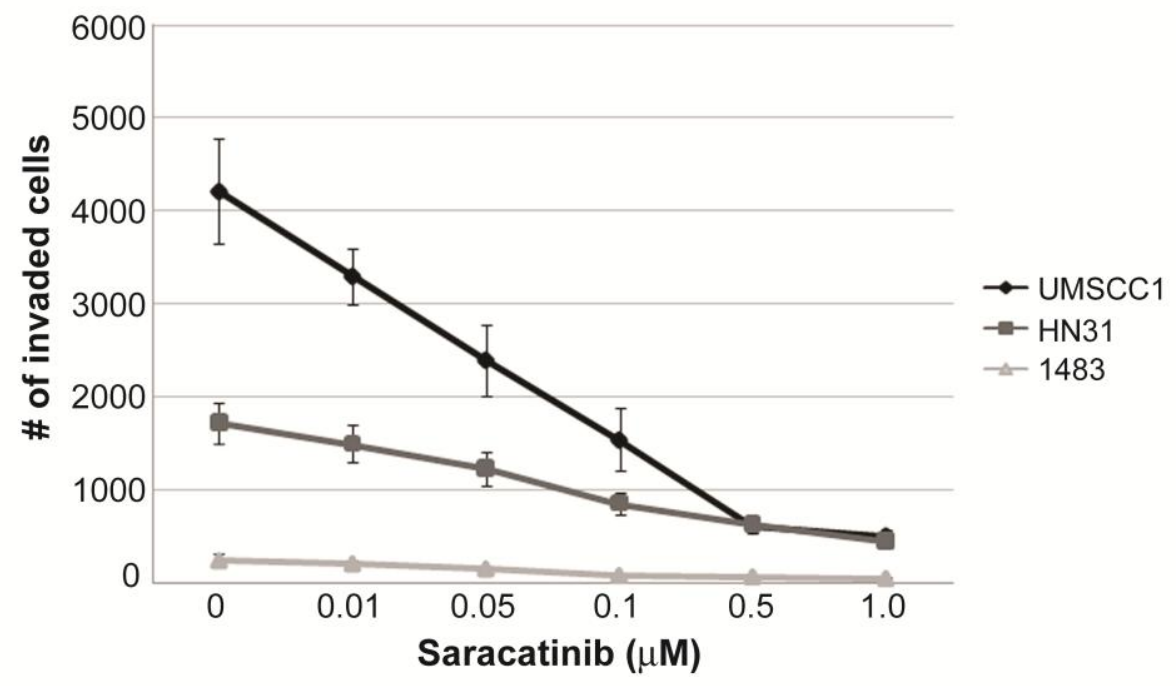

Ammer et al. Figure 1 


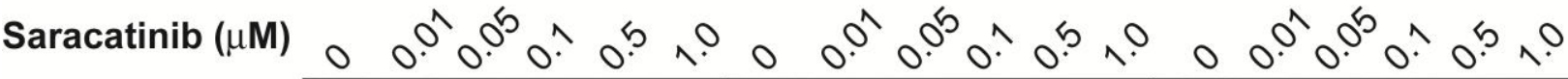

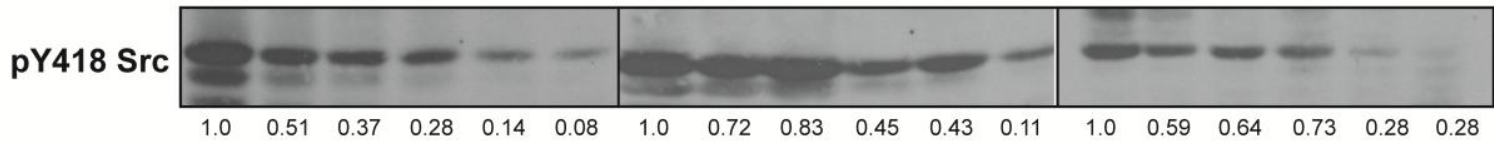

Src
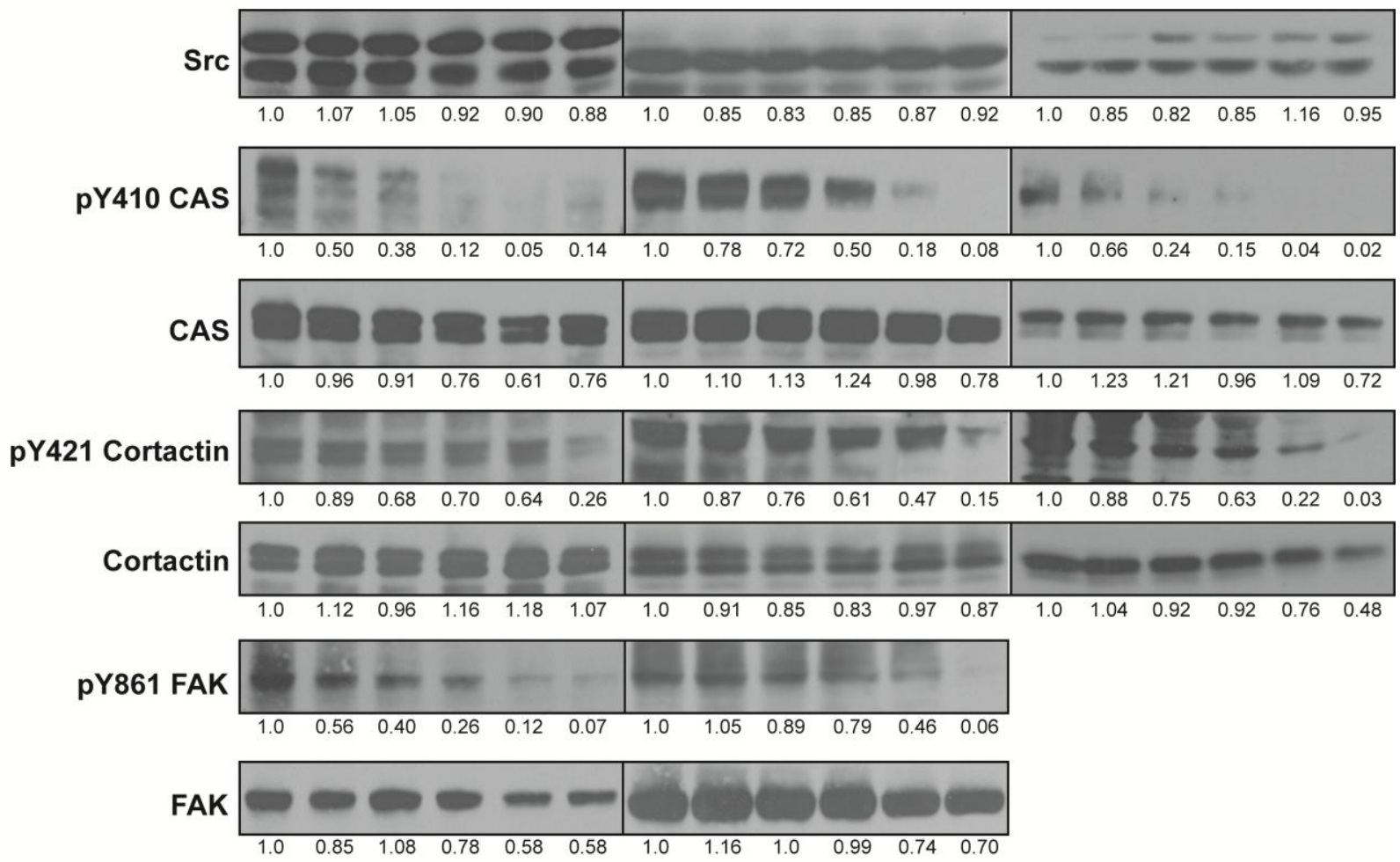

Ammer et al. Figure 2 


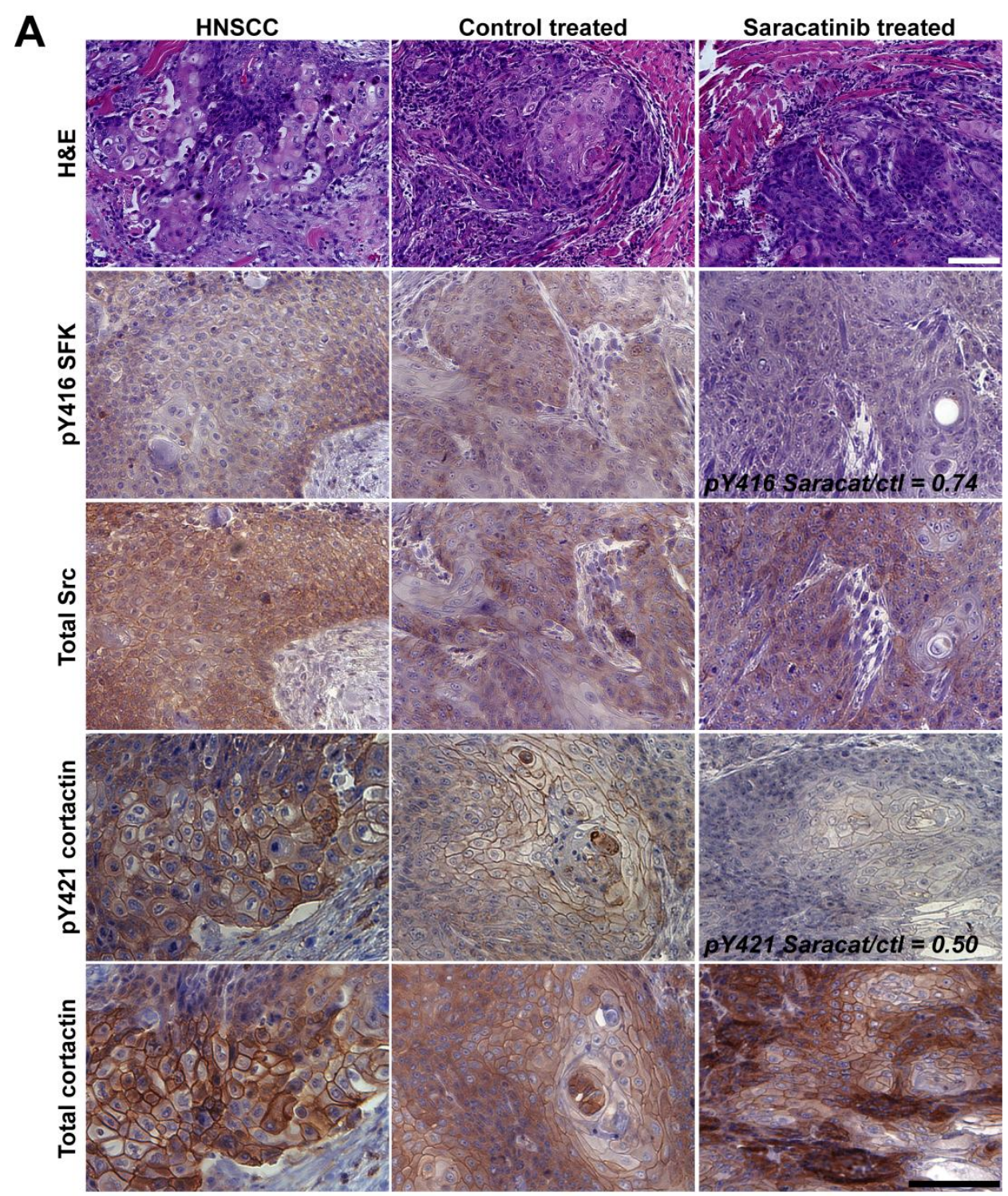

B
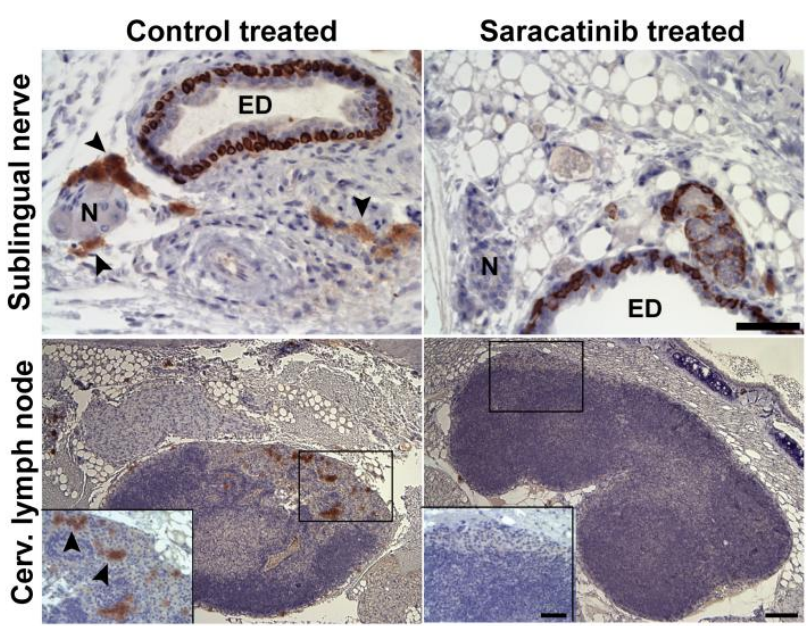

Ammer et al. Figure 3 

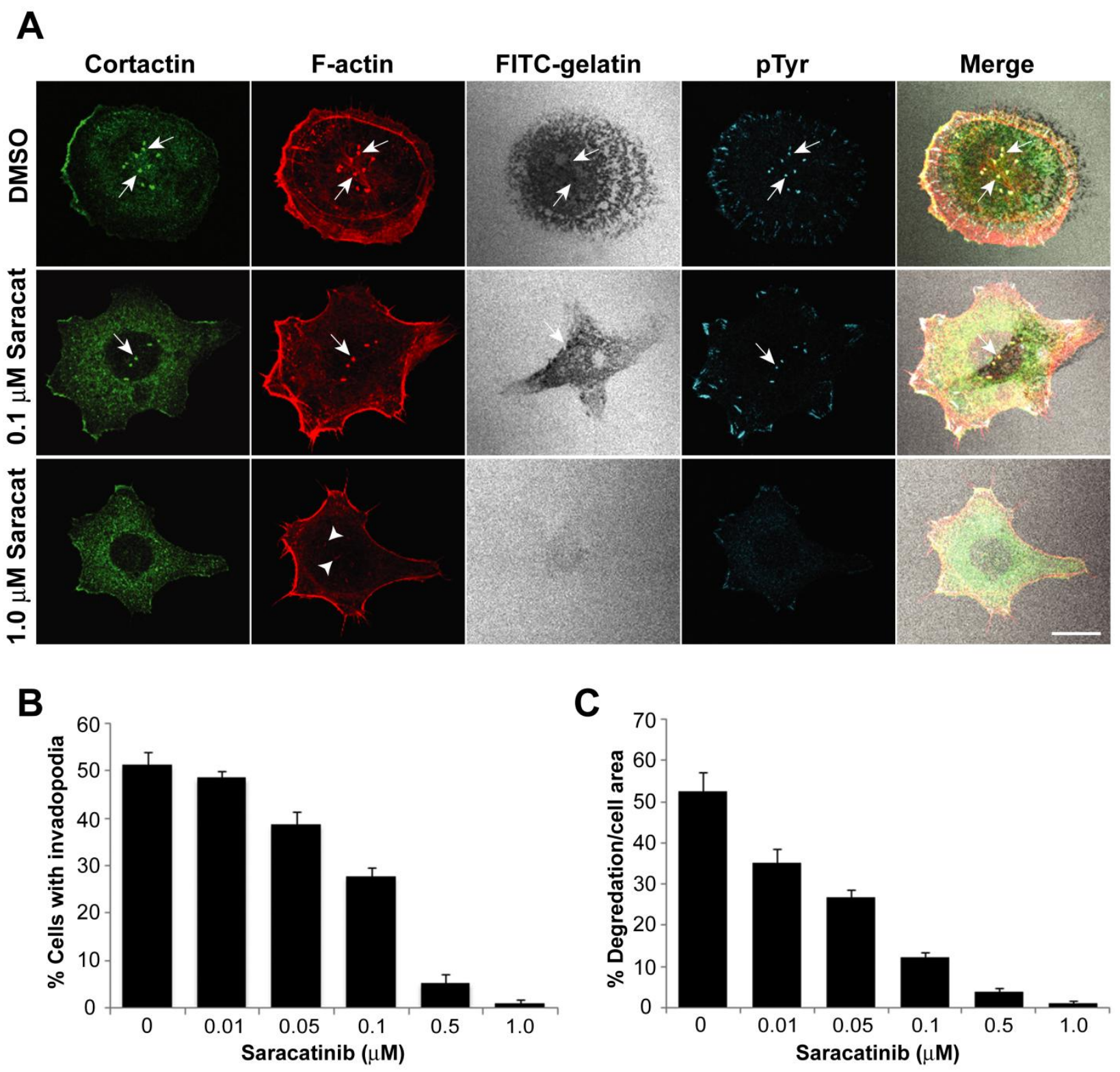

Ammer et al. Figure 4 
A

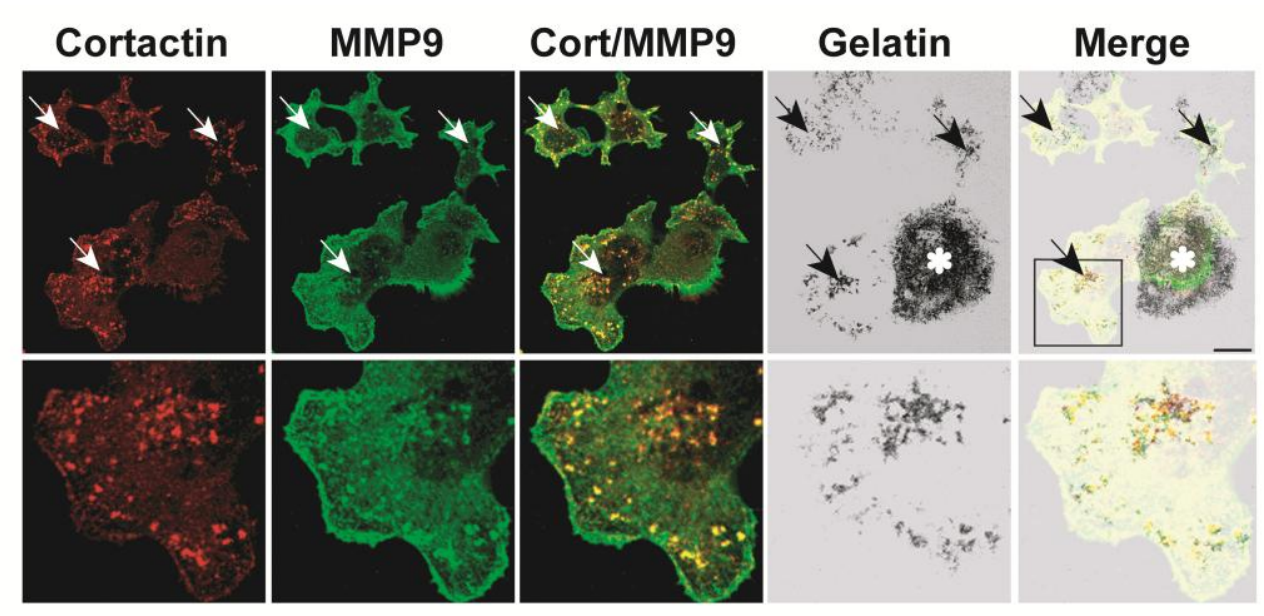

B

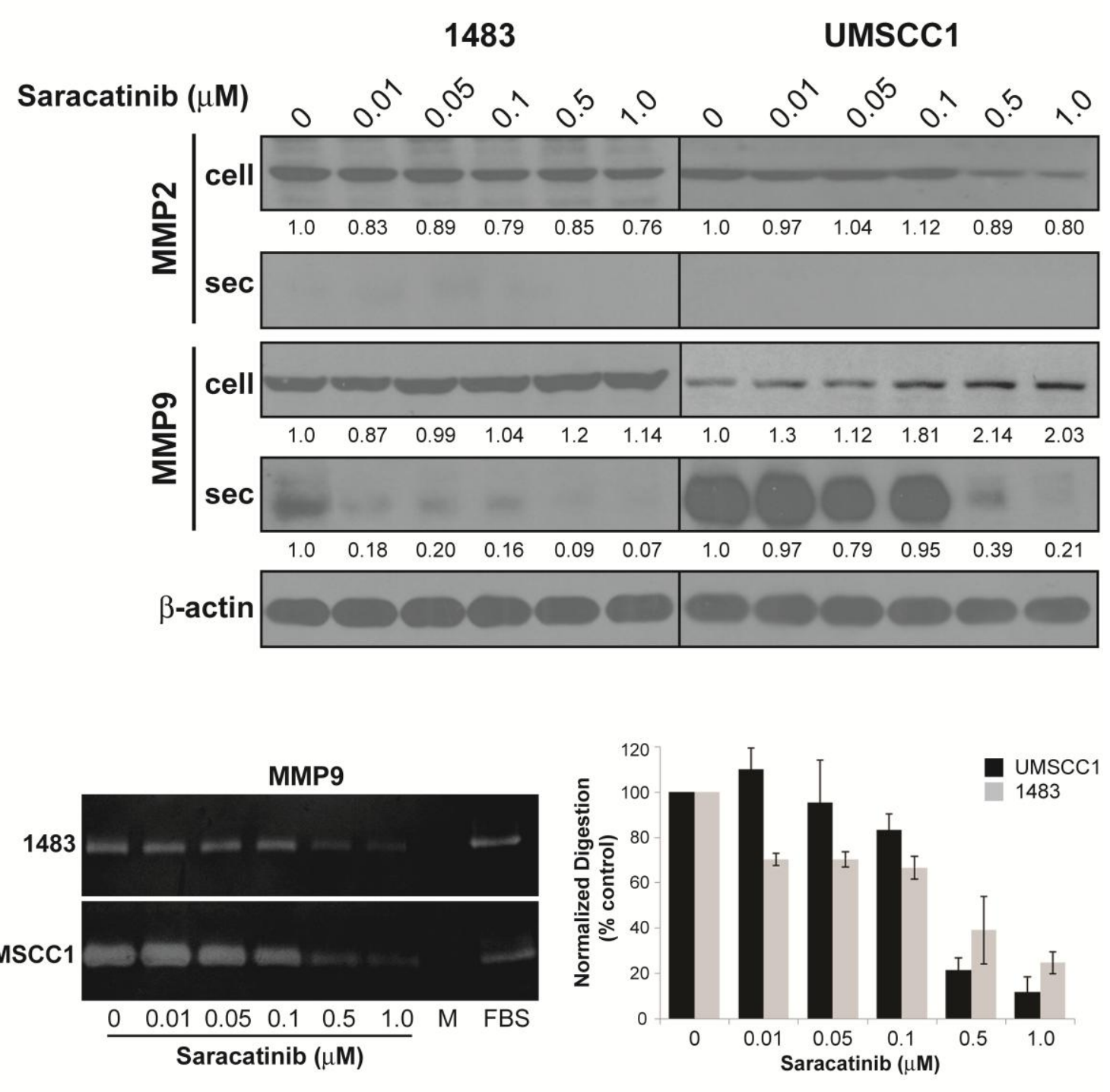

Ammer et al. Figure 5 


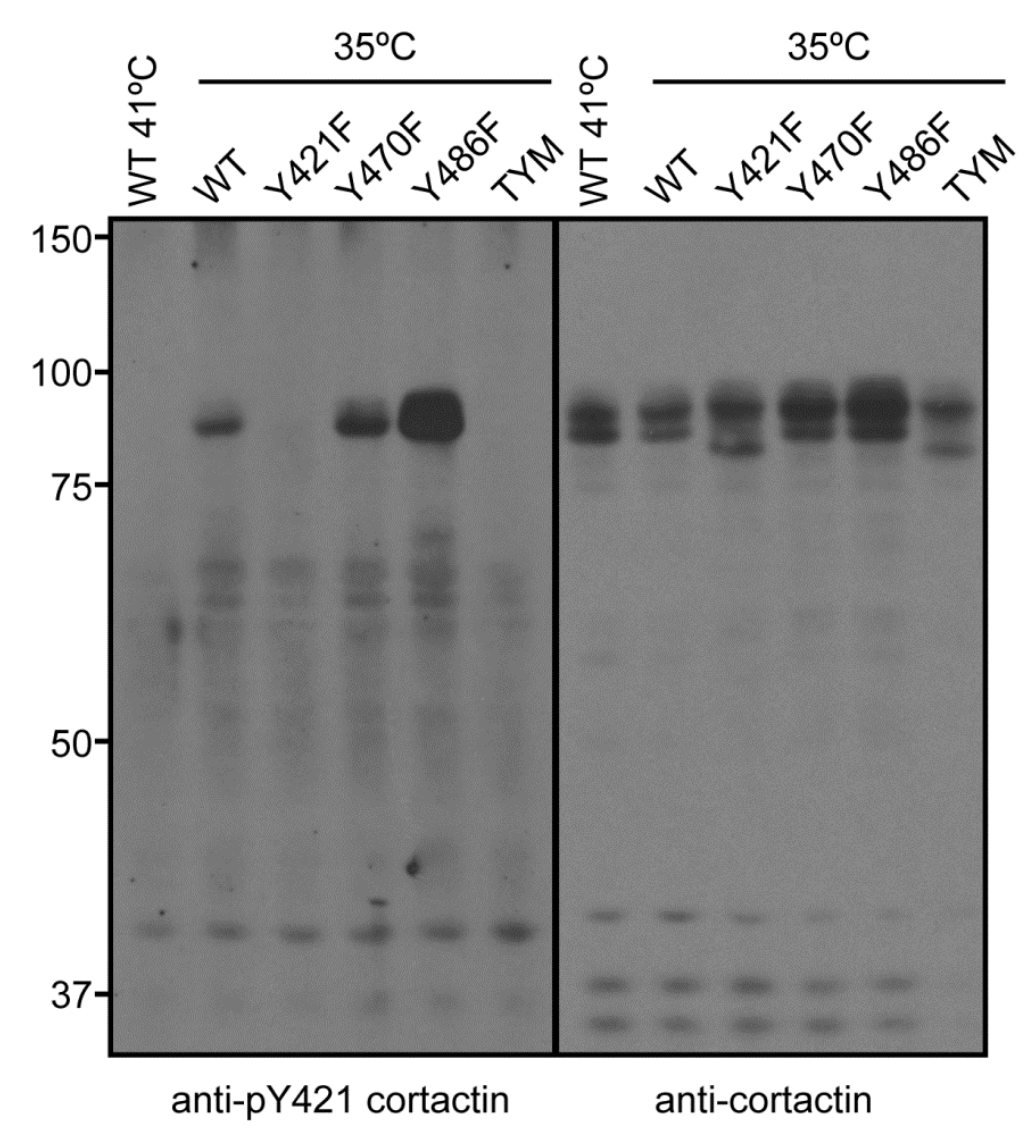

Ammer et al. Supplementary Figure 1

\section{Supplementary Figure Legends}

Supplementary Figure 1 Specificity of human anti-pY421 cortactin antibody. SYF fibroblasts lacking Src, Yes and Fyn were cotransfected with a the temperaturesensitive Src allele La29 along with expression vectors encoding recombinant wild-type human cortactin (WT) or cortactin mutants containing tyrosine-phenylalanine substitutions at the indicated Src-targeted codons and held at the non-permissive temperature $\left(41^{\circ}\right)$ or switched to the permissive temperature $\left(35^{\circ}\right)$. TYM; triple tyrosinephenylalanine cortactin mutant lacking all three Src-targeted sites. Cells were lysed and analyzed by Western blot analysis with anti-pY421 cortactin (left). The blot was stripped and reprobed with anti-cortactin monoclonal antibody 4F11 (right). The position of molecular weight markers is noted on the left in kilodaltons. 


\title{
Study 3: Cortactin Phosphorylated by ERK1/2 Localizes to Sites of Dynamic Actin Regulation and is Required for Carcinoma Lamellipodia Persistence
}

\author{
Laura C. Kelley ${ }^{1,2}$, Karen E. Hayes ${ }^{1,2}$, Amanda Gatesman Ammer ${ }^{1,2}$, Karen H. Martin ${ }^{1}$ \\ and Scott A. Weed ${ }^{1 *}$ \\ ${ }^{1}$ Department of Neurobiology and Anatomy, Program in Cancer Cell Biology, Mary Babb \\ Randolph Cancer Center, West Virginia University, Morgantown, West Virginia, United \\ States of America
}

Published in Plos One 2010 Nov;5(11):e1384, 1-13.

${ }^{*}$ Corresponding author:

Scott A. Weed

West Virginia University

Mary Babb Randolph Cancer Center

Morgantown, WV 26506-9300

Phone: 304-293-3016

Fax: 304-293-4667

Email: sweed@hsc.wvu.edu

${ }^{2}$ Note: These authors contributed equally to this manuscript

Running title: ERK1/2 regulates cortactin 


\section{Abstract}

\section{Background}

Tumor cell motility and invasion is governed by dynamic regulation of the cortical actin cytoskeleton. The actin-binding protein cortactin is commonly upregulated in multiple cancer types and is associated with increased invasion and metastasis. Cortactin regulates actin nucleation through the actin related protein (Arp)2/3 complex, stabilizing the cortical actin cytoskeleton. Cortactin is regulated by multiple phosphorylation events, including phosphorylation of $\mathrm{S} 405$ and $\mathrm{S} 418$ by extracellular regulated kinase (ERK)1/2. ERK1/2 phosphorylation of cortactin has emerged as an important positive regulatory event, enabling cortactin to bind and activate the Arp2/3 regulator neuronal Wiskott-Aldrich syndrome protein (N-WASp), promoting actin polymerization and enhancing cell migration and tumor cell invasiveness.

\section{Methodology/Principal Findings}

In this report we have developed phosphorylation-specific antibodies against cortactin phosphorylated at S405 and S418 to analyze the subcellular localization of this cortactin form in tumor cells and patient samples by microscopy. We evaluated the interplay between cortactin S405 and S418 phosphorylation with cortactin tyrosine phosphorylation in regulating cortactin conformational forms by Western blotting.

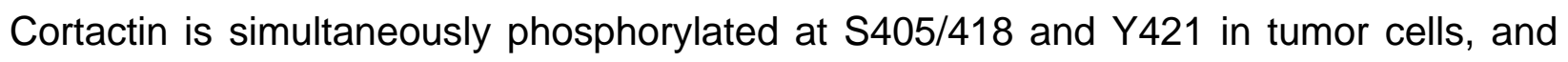
through the use of point mutant constructs we determined that serine and tyrosine phosphorylation events lack any co-dependency. Expression of S405/418 phosphorylation-null constructs impaired carcinoma motility and adhesion, and also inhibited lamellipodia persistence monitored by live cell imaging.

\section{Conclusions/Significance}

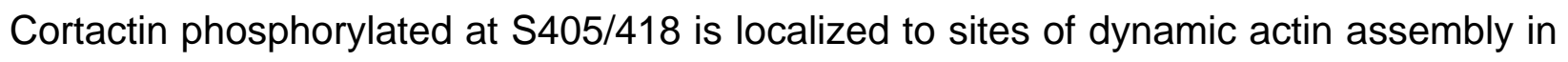
tumor cells. Concurrent phosphorylation of cortactin by ERK1/2 and tyrosine kinases enables cells with the ability to regulate actin dynamics through N-WASp and other effector proteins by synchronizing upstream regulatory pathways, confirming cortactin 
as an important node in actin-based signal transduction. Reduced lamellipodia persistence in cells with S405/418A expression identifies an essential motility-based process reliant on ERK $1 / 2$ signaling, providing additional understanding as to how this vital signaling pathway impacts tumor cell migration. 


\section{Introduction}

Tumor cell motility and invasion is a central problem in cancer that is paramount in contributing to metastasis [1]. Tumor cells move through successive series of coordinated and integrated stages, with formation of protrusive membranous structures including filopodia, invadopodia and lamellipodia required for initiation and maintenance of invasion and migration [2,3,4,5]. Central to the movement of most carcinoma cell types undergoing single or collective migration is the production of lamellipodia at the leading edge of the cell. Lamellipodia are planar protrusive extensions of the plasma membrane produced by motile cells in two- and three-dimensional settings [6]. Lamellipodia extension drives cell migration through integrin-based adhesion with the underlying substratum, providing the necessary traction for contractile-based translocation of the cell body to generate productive movement [7]. It is generally accepted that dynamic regulation of the cortical actin cytoskeleton through cycles of actin polymerization and depolymerization are responsible for generating the propulsive force needed for lamellipodia extension [8].

The actin cytoskeleton within lamellipodia is governed by the activity of numerous actinbinding proteins. One element central to the formation of lamellipodia actin networks is activation of the actin-related (Arp) 2/3 complex, which nucleates filamentous ( $F-$-) actin polymerization within lamellipodia [9]. Arp2/3 complex binds to the sides of pre-existing F-actin, where upon activation the Arp2 and Arp3 subunits mimic the fast growing ("barbed" or "+") end of an actin filament, allowing for the rapid addition of actin monomers to the complex and subsequent filament extension [4]. The resulting Arp2/3F-actin networks comprise an organized branched array of F-actin filaments that contribute to lamellipodia extension, with Arp2/3 localized at filament branch points [8,9]. Arp2/3 branch points are metastable, allowing for rapid breakdown of Arp2/3-Factin networks by filament debranching [10]. Debranched F-actin filaments are further disassembled through the severing activity of members of the actin depolymerizing factor/cofilin family, which ultimately dissolve F-actin filaments to promote depolymerization, recycling actin monomers for additional rounds of polymerization and lamellipodia extension [11]. 
Arp2/3 activation is controlled by the activity of several actin nucleation promoting factors (NPFs). The best characterized NPFs to date are members of the WiskottAldrich syndrome protein (WASp) family, which include the WASp and WAVE protein subgroups $[12,13]$. In many cell types, regulation of Arp2/3 activity by the WASp proteins N-WASp and WAVE2 are largely responsible for generating the actin network used for creating and regulating lamellipodia, filopodia and invadopodia [4,12,14], making these NPFs critical mediators of cell motility and invasion.

Another well-characterized NPF independent of the WASp protein family is the cortical actin-binding protein cortactin [15,16]. Cortactin directly binds Arp2/3 complex $[17,18]$ and activates Arp2/3 complex nucleation activity in vitro, albeit at a lesser degree than WASp-family proteins $[18,19]$. In addition to actin nucleation, a unique function of cortactin is its ability to prevent spontaneous debranching of Arp2/3-F-actin networks by simultaneous binding to Arp2/3 and F-actin, prolonging the lifetime of branched filaments [19]. While the biochemical features of cortactin seem to point to a straightforward role in lamellipodia actin regulation, studies of cortactin function in lamellipodia have proven controversial, suggesting to a more complex role in cell migration. For instance, RNA interference studies have yielded conflicting results in regards to lamellipodia dynamics, with cortactin knockdown resulting in decreased lamellipodia stability and reduced persistence [20,21,22], whereas similar studies in different cell types suggest cortactin downregulation increases the length of extending lamellipodia [23]. Furthermore, recent analysis of fibroblast lamellipodia dynamics in cortactin-/- cells indicates that cortactin does not play a role in directly regulating lamellipodia protrusion or Arp2/3-based actin dynamics, but rather is important in mediating upstream activation of the small GTPases Rac1 and Cdc42, which in turn regulate WAVE2 and N-WASp activity [24]. While these reported discrepancies regarding cortactin function in lamellipodia have yet to be fully reconciled, it is clear that cortactin is an important regulator for normal and tumor cell migration in many cell systems [25,26]. An unambiguous role for cortactin has been shown in invadopodia, where removal of cortactin by RNA interference ablates invadopodia formation in multiple invasive tumor cell types $[27,28,29]$. 
Besides regulating Arp2/3-based cortical actin networks by direct interactions, cortactin also functions as a key mediator in several kinase-based signal transduction cascades that serve to indirectly govern Arp2/3 activity and subsequent cell movement. Cortactin is a well-defined target for Src kinase [30], phosphorylating human cortactin on tyrosine residues Y421, Y470 and Y486 within the proline-rich (PR) carboxyl-terminal domain [31]. Several other tyrosine kinases target these residues [32,33,34,35], indicating that they collectively form a "hot spot" region as a point of convergence for multiple signaling pathways. Cortactin phosphorylated at tyrosines 421,470 and/or 486 creates Src homology $(\mathrm{SH}) 2$ docking sites for several phosphorylating kinases, as well as the adaptor proteins Crk [36] and Nck1 [37]. In the case of Nck1, Nck1/cortactin complexes interact with N-WASp or WASp interacting protein through the Nck1 SH3 domain to stimulate Arp2/3-dependent actin nucleation [37], which in cooperation with Arg kinase has been recently demonstrated to be important for regulating lamellipodia protrusion and leading edge adhesion formation [38]. The cortactin/Nck1 complex is also required to stimulate actin polymerization essential for invadopodia function in invasive breast cancer cells [39]. These studies are in agreement with the localization of tyrosine phosphorylated cortactin within lamellipodia and invadopodia [40], lending mechanistic insight into the long recognized pro-migratory and pro-invasive properties associated with cortactin tyrosine phosphorylation $[29,31,41,42]$.

In addition to tyrosine phosphorylation, cortactin is a target for multiple serine/threonine kinases [43]. Stimulation of tumor cells with epidermal growth factor (EGF) results in increased serine/threonine phosphorylation of serine residues 405 and 418 within the PR domain, coincident with a characteristic shift in cortactin electrophoretic mobility from $80 \mathrm{kDa}$ to $85 \mathrm{kDa}$ in SDS-PAGE [44,45]. The mobility shift and phosphorylation of S405/S418 are impaired by pharmacologic inhibition of mitogen activated protein/extracellular signal regulated kinase kinase (MEK)1/2, and direct biochemical evidence indicates that the MEK effector kinases ERK1/2 directly phosphorylate cortactin at these sites [45]. Phosphorylation of S405/S418 enhances binding of the cortactin carboxyl-terminal SH3 domain to N-WASp and activates N-WASp NPF activity, indicating a functional role in stimulating Arp2/3-mediated actin dynamics independent of tyrosine phosphorylation [46]. This is supported by studies expressing 
phosphorylation-null and phosphomimetic point mutant constructs in cells, suggesting that S405/S418 phosphorylation plays a critical role in regulating cellular actin polymerization necessary to promote cell motility [47] and invadopodia function [29]. In addition, p21 activated kinase 1 (PAK1) phosphorylates cortactin at S405/S418, serving to stimulate N-WASp activity required for clathrin-independent endocytosis [48]. While studies to date implicate a positive regulatory function for cortactin S405/418 phosphorylation in promoting N-WASp-mediated Arp2/3 actin structures, the subcellular localization of phosphorylated S405/418 cortactin, as well as the precise role S405/418 phosphorylation plays in regulating lamellipodia dynamics have not been evaluated.

In this study, we have generated site-specific antibodies against phosphorylated cortactin S405 and S418 to determine the spatial and temporal localization of cortactin in dynamic actin structures and human tumors, and to evaluate signaling interplay between cortactin tyrosine and serine phosphorylation events. We also determined the effects of S405/418 cortactin phosphorylation on EGF-induced cell migration, adhesion and lamellipodia dynamics in carcinoma cells. 


\section{Materials and Methods}

\section{DNA Constructs and siRNA}

For Myc-tagged human cortactin expression constructs, the wild-type human cortactin cDNA subcloned into pcDNA FLAG2AB [49] was used as a template for producing point mutants by site-directed mutagenesis (QuickChange; Stratagene, La Jolla, CA). Codon alterations in human cortactin were: S405A, S418A, S405A/S418A, Y421F, Y470F, Y486F, Y421F/Y470F/Y486F and W492K. Cortactin cDNAs were amplified by PCR as BamHI-EcoRI fragments and subcloned into BamHI-EcoRI digested pRK5Myc [50]. Murine GFP-tagged expression constructs were produced using pcDNA3FLAG2AB wild-type murine cortactin [17] as the template for mutagenesis, then subcloned as EcoRI-Kpnl PCR fragments into pAcGFP-C1 (Clontech, Mountain View, CA). The temperature-sensitive vSrc LA29 construct was previously described [51]. mCherry- $\beta$ actin was obtained from D. Schafer (University of Virginia), with the parent construct produced by R. Tsien (University of California, San Diego). Small interfering (si)RNA targeting rodent cortactin (5'-GCTTCGAGAGAATGTCTTC-3') was purchased from Thermo Scientific (Waltham, MA)

\section{Cell lines and Transfection}

The HNSCC cell lines 1483 [52], UMSCC1 and UMSCC2 [53] were maintained as described [41]. SYF cells were obtained from the American Type Culture Collection (Manassas, VA) and maintained according to the supplied protocol. The rat mammary adneocarcinoma line MTLn3 was maintained in aMEM supplemented with $10 \%$ fetal bovine serum, $1 \% \mathrm{~L}$-glutamine and $1 \%$ penicillin-streptomycin. Transient transfections were conducted with $3 \times 106$ cells and $2 \mu \mathrm{g}$ of plasmid construct or siRNA using the Nucleofector I device (Amaxa Biosystems, Berlin, Germany).

\section{Antibodies}

Antibodies against phosphorylated serine 405 (pS405) and serine 418 (pS418) of human cortactin were produced by $21^{\text {st }}$ Century Biochemicals (Marlboro, MA). Synthetic phosphorylated cortactin peptides containing the sequences $\mathrm{NH}_{2}$ - 
KTQTPPV[pS]PAPQPTC-COOH (cortactin pS405) and $\mathrm{NH}_{2}$-TEERLPS[pS]PV-COOH (cortactin pS418) were produced, conjugated to keyhole limpet cyanine and injected into rabbits. Immune serum was screened by enzyme-linked immunosorbent assay against the appropriate phosphorylated cortactin peptide coupled to bovine serum albumin. High-titer bleeds were identified for each peptide, and immune serum was passed two successive times through chromatography columns containing agarose beads coupled to the equivalent non-phosphorylated peptide. The flow through material for each peptide was subsequently passed twice through chromatography columns containing beads conjugated to the matched phosphorylated cortactin peptide. After extensive washing, bound antibodies for each phosphorylation site were eluted, concentrated and screened for specificity by Western blotting against recombinant cortactin mutant proteins harboring alanine-serine point mutations at serine 405 or 418 , respectively (Fig. 1A). The anti-pS405 and anti-pS418 cortactin antibodies are currently available through Protea Biosciences (Morgantown, WV). Anti-cortactin (4F11) was used as described [41]. Anti-pY421 cortactin and anti-pY418 Src were from Invitrogen (Carlsbad, CA). Anti-ERK1/2 and pERK1/2 were from Cell Signaling (Danvers, MA). Anti-Myc epitope tag (4A6) was from Millipore (Billerica, MA). Anti-GFP (JL-8) was fromClontech (Mountain View, CA) and anti- $\beta$-actin was from EMD4Biosciences (San Diego, CA).

\section{Western blotting and Immunoprecipitation}

Western blotting was conducted as described [41]. Primary antibody dilutions used were: anti-pS405 cortactin (1:4000), anti-pS418 cortactin (1:500), anti-cortactin (1:1000), anti-pY-421 cortactin (1:2000), anti-ERK1/2 (1:2000), anti-pERK (1:2000), anti-pY418 Src, anti-GFP (1:1000) and anti- $\beta$-actin (1:5000). Immunoprecipitations were performed as described [40] using $5 \mu \mathrm{g}$ of precipitating antibody captured with $40 \mu \mathrm{l}$ of a $50 \%$ Protein $A / G$ bead slurry (Thermo Fisher Scientific, Pittsburgh, PA). In some cases cells were treated with selumetinib (AZD6244; ARRY-142886) or saracatinib (AZD0530) for $24 \mathrm{~h}$ prior to immuoprecipitation and Western blotting analysis. 


\section{Microscopy}

UMSCC2 cells were plated on fibronectin-coated coverslips $(10 \mu \mathrm{g} / \mathrm{ml}$; Sigma, St Louis, $\mathrm{MO}$ ) and allowed to attach before serum starvation for $16 \mathrm{~h}$. Cells were stimulated with 100ng/ml EGF (Millipore) for $1 \mathrm{~h}$ before fixation. UMSCC1 cells plated on FITC-gelatin (Sigma) for $8 \mathrm{~h}$ were processed for confocal microscopy using Zeiss LSM 510 Meta system (Thornwood, NY) as described [49]. Anti-pS418 cortactin was used at 1:1000, 4F11 at 1:500 and rhodamine-conjugated phalloidin at 1:1000 (Invitrogen, Carlsbad, $\mathrm{CA})$.

For immunohistochemistry, HNSCC tissue blocks were obtained from the West Virginia University Tissue Bank and used under approval of the West Virginia University Institutional Review Board. Five-micrometer sections from formalin-fixed, paraffinembedded blocks were processed for immunostaining using the Discovery XT automated staining system (Ventana, Tucon AZ). Briefly, after deparaffinization and antigen retrieval, sections were incubated with monoclonal rabbit anti-cortactin (Novus, Littleton, CO) at 1:2000, anti-pS418 cortactin at 1:25 and anti-pERK1/2 at 1:100 dilutions. All primary antibodies were incubated in Dako diluent (Dako, Carpinteria, CA) for $1 \mathrm{~h}$. Primary antibodies were detected with the Omnimap antibody horseradish peroxidase kit (Ventana). Slides were counterstained with hematoxylin and postcounterstained with bluing reagent (Ventana). Images were visualized with an Olympus AX70 microscope and captured using the MicroBrightfield system (Williston, VT).

Live cell imaging was conducted using MTLn3 cells starved for $3 \mathrm{~h}$ with serum-free media prior to stimulation with $100 \mathrm{ng} / \mathrm{ml}$ EGF. Cells were plated on delta-T glass bottom dishes (Fisher) coated with $10 \mu \mathrm{g} / \mathrm{ml}$ fibronectin (Sigma). Immediately following EGF addition, cells were imaged by differential interference contrast using a Nikon TE2000 inverted microscope equipped with a Roper CoolSNAP HQ charge-coupled device camera (Photometrics, Tucson, AZ). Images were captured every $5 \mathrm{~s}$ for $15 \mathrm{~min}$ (181 total frames). A Nikon LiveScan SFC swept field microscope was used for imaging cells expressing mCherry-actin. In all cases, GFP-cortactin expressing cells were identified by fluorescence microcopy prior to imaging. Kymograms were produced by 
extracting 1 pixel-width strips from each movie frame at points of initial and maximal lamellipodia extension, and assembled using ImageJ (v1.40).

\section{Electric Cell Substrate Impedance Sensing}

To assay cell motility and adhesion, $5 \times 10^{5}$ cells were plated into 8-well electric cell substrate impedance sensing dishes (ECIS; Applied Biophysics, Troy, NY). For motility measurements, cells were allowed to adhere overnight on 8W1E dishes to form a monolayer. Adhesion was assayed immediately after plating cells onto 8W10E dishes. Measurements were conducted for $24 \mathrm{~h}$ at $45 \mathrm{kHz}$, with reading taken at $1 \mathrm{~min}$ intervals. Cells treated with selumetinib were serum starved $24 \mathrm{~h}$ in the presence of drug prior to ECIS.

\section{Statistical Analysis}

Differences in mean groups for migration, adhesion and kymography between control and treated groups were evaluated using one way ANOVA, followed by StudentNewman-Keuls post hoc testing. All differences were considered significant at $p<0.05$. A minimum of three experimental groups were used for all analyses. 


\section{$\underline{\text { Results }}$}

\section{Localization of pS418 cortactin with dynamic cortical actin structures}

We developed antibodies specific to phosphoserine 405 (pS405) and phosphoserine 418 (pS418) of human cortactin to facilitate analysis of these sites. To validate antibody specificity, epitope (Myc)-tagged cortactin constructs containing wild-type (WT) cortactin, cortactin with individual serine to alanine mutations at serine 405 (S405A), 418 (S418A) or with both mutated in tandem (S405,418A) were produced and transfected into 1483 cells. Total cell lysates were blotted with anti-pS405 or antipS418 antibodies (Figure 1A). The anti-pS405 antibody recognized the WT and S418A cortactin variants, failing to blot constructs containing the S405A mutation. Conversely, anti-pS418 blotted WT and S405A, failing to recognize cortactin constructs with S418A mutations. All cortactin variants were recognized by an anti-cortactin monoclonal antibody (Figure 1A), indicating equivalent expression of the assayed constructs. These results indicate that the anti-pS405 and anti-pS418 antibodies specifically recognize their cognate phosphorylated cortactin epitope, and that no interdependence exists between phosphorylation of cortactin S405 and S418.

To determine the subcellular localization of serine phosphorylated cortactin, we conducted indirect immunofluoresence studies on cells producing lamellipodia and invadopodia, two actin-based structures that depend in part on N-WASp activity. While the anti-pS405 antibody yielded non-specific staining in our hands (data not shown), anti-pS418 specifically labeled lamellipodia and cytoplasmic puncta (presumably vesicles) in UMSCC2 cells. In cells with a motile phenotype, anti-pS418 localized with cortactin and F-actin in these regions (Figure 1B, top row). Labeling of UMSCC1 cells plated on FITC-coated gelatin matrix with anti-pS418 indicated specific localization to a subset of invadopodia that coincided with cortactin, F-actin and areas of gelatin clearing indicative of matrix metalloproteinase mediated invadopodia activity (Figure 1B, middle and bottom rows). 
In solid human tumors, cortactin and cortactin phosphorylated on tyrosine 421 (pY421) localizes to invasive tumor fronts and to cell-cell junctions $[41,49]$. To determine the location of pS418 cortactin in tumor tissue, head and neck squamous cell carcinoma (HNSCC) cases were sectioned and stained with anti-pS418 (Figure 1C). Cortactin pS418 was abundant in HNSCC cell cytoplasm and was enriched in areas of cell-cell contact, displaying a pattern similar to sections labeled with a total cortactin antibody (Cort). These tumor regions also contained activated ERK1/2, as evidenced by pronounced cytoplasmic and nuclear staining of phosphorylated ERK1/2 in serial sections (Figure 1C).

\section{Growth factor mediated phosphorylation of cortactin S405/418 is MEK dependent}

Previous biochemical work has implicated chemical inhibition of MEK and subsequent blocking of ERK1/2 activation as a major pathway responsible for cortactin S405/418 phosphorylation [45]. To further evaluate the role of the MEK-ERK1/2 pathway on cortactin phosphorylation, we utilized the anti-pS405 and pS418 cortactin antibodies to directly test the effects of MEK inhibition on cortactin pS405/418. Western blot analysis of cell extracts from EGF- and serum-stimulated UMSCC1 cells with anti-pS405 and pS418 antibodies displayed similar phosphorylation kinetics of S405 and S418, with phosphorylation of both sites first evident $10 \mathrm{~min}$ after stimulation (Figure 2A) and remaining phosphorylated up to $2 \mathrm{~h}$ (data not shown). Treatment of UMSCC1 or 1483 cells with the small molecule MEK inhibitor selumetinib [54] reduced EGF-stimulated cortactin S405/418 phosphorylation in a dose-dependent manner, where near elimination of phosphorylation at both serine residues occurred at doses $\geq 1 \mu \mathrm{M}$ (Figure 2B). ERK1/2 activity was also reduced under similar dose conditions, although complete ablation of ERK1/2 phosphorylation was observed at doses $\geq 5 \mu \mathrm{M}$ (Figure $2 \mathrm{~B})$. These data suggest that the MEK-ERK pathway is largely responsible for growthfactor induced cortactin S405/418 phosphorylation in HNSCC cells, in agreement with previous findings in other cell types [45]. 
The $80 \mathrm{kDa}$ to $85 \mathrm{kDa}$ cortactin conformational shift is associated with serine and tyrosine phosphorylation

Based on sequence analysis, the largest and most prominent cortactin isoform (cortactin "A" or "SV1") encodes a $61.5 \mathrm{kDa}$ protein $[55,56]$. This cortactin form frequently migrates as an $80 / 85 \mathrm{kDa}$ doublet in SDS-PAGE $[30,57]$ that has been attributed to conformational alterations within the polypeptide chain [15,45]. Shifting from the $80 \mathrm{kDa}$ to $85 \mathrm{kDa}$ form is seen in response to EGF, with the resulting $85 \mathrm{kDa}$ band associated with $5405 / 418$ phosphorylation [44,45]. To directly assess the presence of $\mathrm{pS} 405 / 418$ in the two cortactin conformational isomers, serum-starved UMSCC2 (Figure 3A) and 1483 (Figure 3B) cells were stimulated with EGF and the cortactin forms in cell lysates were analyzed at successive time points with anti-pS405 and anti-pS418 antibodies. S405/418 phosphorylation was maintained in the $85 \mathrm{kDa}$ cortactin form in both cell lines following serum starvation, despite of the lack of ERK1/2 activity ( $0 \mathrm{~min}$, Figure $3 \mathrm{~A}$ and Figure $3 \mathrm{~B}$ ). EGF stimulation resulted in complete conversion of the $80 \mathrm{kDa}$ to the $85 \mathrm{kDa}$ cortactin form by $1 \mathrm{~h}$ after EGF treatment in both cell lines (Figure 3A and Figure 3B). Cortactin pS405 and pS418 was observed primarily in the $85 \mathrm{kDa}$ form and increased at both sites during the entire time course, whereas ERK1/2 activity peaked at $15 \mathrm{~min}$ and rapidly declined afterwards (Figure 3A and Figure 3B). Interestingly, the phosphorylation of S405 was also associated with an increase appearance of cortactin degradation in UMSCC2 cells (Figure $3 \mathrm{~A}$ ). It is uncertain whether these products represent increased overall cortactin degradation, or if the net cortactin degradation is constant but is selectively identified by the pS405 antibody in response to EGF treatment and phosphorylation. EGF-induced Src activation and cortactin pY421 phosphorylation was sustained throughout the entire time course in UMSCC2 cells (Figure 3A), indicating that cortactin can be simultaneously phosphorylated by ERK $1 / 2$ and Src or potentially other EGF-stimulated cortactin tyrosine kinases. Pretreatment of UMSCC2 cells with the Src family kinase inhibitor saracatinib at $10 \mu \mathrm{M}$ or selumetinib at $1 \mu \mathrm{M}$ completely impaired the cortactin shift from $80 \mathrm{kDa}$ to $85 \mathrm{kDa}$ (Figure $3 \mathrm{C}$ ). The exclusive presence of pS405 and pS418 in the EGF-induced $85 \mathrm{kDa}$ cortactin form, as well as the ability of MEK inhibition to impair the cortactin shift is consistent with results obtained from previous work [45]. Our 
results also identified EGF-induced Src-mediated phosphorylation of cortactin at tyrosine 421 as a necessary mediator of the cortactin shift.

\section{Cortactin serine phosphorylation in vivo is independent from tyrosine phosphorylation}

EGF treatment of UMSCC2 cells resulted in phosphorylation of cortactin S405/418 and cortactin pY421 (Figure 3A). A previous in vitro study evaluating the impact of cortactin phosphorylation on N-WASp activation determined that $S 405 / 418$ phosphorylation by ERK1/2 enables the cortactin SH3 domain to stimulate N-WASp Arp2/3 activation, while Src phosphorylation downregulates N-WASp activity and counteracts the effects of S405/418 phosphorylation [46]. This proposed "on-off switch" postulates that cortactin serine and tyrosine phosphorylation are mutually exclusive events governing the ability of cortactin to regulate N-WASp activity and downstream actin reorganization [58]. Using the available antibodies reactive against cortactin pS405 and pY421, we sought to determine if these two different classes of phosphorylation events are interdependent in any manner. Cortactin depleted SYF fibroblasts (null for the Src, Yes and Fyn kinases) were co-transfected with the temperature-sensitive vSrc construct $t s$ La29-GFP [51] to activate the Src and ERK1/2 signaling pathways, along with constructs encoding wild-type cortactin or the following Myc-tagged cortactin mutants: Y421F, Y470F, Y486F, Y421/Y470/Y486F (TPM), S405A, S418A, S405/418A (Figure 4A). A W492K cortactin mutant was also included, as this mutant abolishes the ability of the cortactin $\mathrm{SH} 3$ domain to interact with corresponding SH3 binding proteins [59]. After shifting to $35^{\circ} \mathrm{C}$ for $2 \mathrm{~h}$ to activate tsLa29-GFP, the serine and tyrosine cortactin mutants were analyzed for phosphorylation at Y421 and S405 by SDS-PAGE and Western blotting (Figure 4B). Mutations to S405 and S418 alone and in combination did not impact the ability of these constructs to be phosphorylated on Y421, as indicated by their recognition with the anti-pY421 antibody (Figure 4B). Similarly, mutations to Y421, Y470, and Y486, alone and in combination (TYM) did not affect the ability of these constructs to be phosphorylated on S405. These data indicate that cortactin is simultaneously phosphorylated at S405 and Y421 downstream of vSrc activation, suggesting in this system that phospho-regulation of cortactin $\mathrm{SH} 3$ domain function is 
not governed in vivo by the serine-tyrosine "on-off switch" mechanism proposed from previous in vitro experimentation $[46,58]$.

\section{S405/418 phosphorylation is required for efficient tumor cell motility and adhesion}

To evaluate the role of cortactin S405/418 phosphorylation on carcinoma cell migration, 1483 and UMSCC1 cells were treated with selumetinib and assayed for effects on motility by ECIS (Figure 5). Selumetinib treatment impaired the motility of both cell types in a dose-dependent manner, corresponding to the observed decreases in S405/418 phosphorylation (Figure 2B). Since MEK inhibition likely impaired the phosphorylation of other proteins involved in motility in addition to cortactin, we directly assessed the impact of cortactin S405/418 phosphorylation on cell migration using phosphorylation-null cortactin expression constructs. MTLn3 rat mammary adneocarcinoma cells were initially transfected with a siRNA targeted against rodent cortactin, followed by transfection with GFP-tagged human wild-type (WT), S405A, S418A and S405/418A cortactin constructs. Cortactin siRNA reduced endogenous cortactin levels to $>90 \%$, having no impact on expression of the human GFP-labeled variants (Figure 6A). MTLn3 cells with cortactin knockdown (si) displayed a $29 \%$ reduction in motility compared to control (Ctl) (Figure 6B). Expression of wild-type human GFP-cortactin (WT) led to a 2-fold increase in motility, presumably due to increased expression of this variant over endogenous (Ctl) levels (Figure 6A). Expression of S405A, S418A or S405,418A cortactin resulted in an $49 \%$ average decrease in cell migration for each cortactin mutant, indicating that phosphorylation of S405 and S418 are both vital in maintaining optimal carcinoma cell motility (Figure 6B). Since lamellipodia formation is required for detached cells to adhere to the ECM, we conducted ECIS assays to determine the effects of cortactin S405/418 phosphorylation on cell adhesion. MTLn3 cells lacking cortactin expression (si) exhibited a 50\% decrease in cell adhesion compared to control (Ctl) cells. Expression of wild type (WT) GFP-cortactin restored adhesion to levels similar to Ctl, whereas expression of S405A, S418A or S405/418A cortactin mutants all reduced adhesion to levels $42-58 \%$ of Ctl, failing to restore adhesion to levels above cortactin si cells (Figure $6 \mathrm{C}$ ). These results 
suggest that $\mathbf{S} 405 / 418$ phosphorylation is critical for carcinoma cell motility and adhesion, representing an important pro-migratory substrate targeted by the MEKERK $1 / 2$ pathway.

\section{Cortactin S405/418 phosphorylation is required for carcinoma cell lamellipodia persistence}

Given the localization of pS418 cortactin within lamellipodia (Figure 1B) and the effects of cortactin S405/418A expression on cell motility, we evaluated the impact of cortactin S405/418 phosphorylation on lamellipodia dynamics using live-cell imaging and kymographic analysis. Serum-starved MTLn3 cells expressing mCherry- $\beta$-actin and containing endogenous cortactin knockdown alone (si), rescued with human GFP- wildtype cortactin (si+WT) or with GFP-cortactin S405/418A (si+S405,418) were stimulated with EGF for $15 \mathrm{~min}$. Lamellipodia dynamics were monitored by time lapse video microscopy (Figures S1-S4) and assayed by kymography (Figure 7A). EGF-stimulated MTLn3 cells produced an initial dominant lamellipodia that reached maximal extension between 1.5 and $3 \mathrm{~min}$, and retracted to the point of origin between $5-7 \mathrm{~min}[60,61]$. Control MTLn3 cells containing mCherry- $\beta$-actin displayed similar extension-retraction kinetics when assayed by kymography (Figure 7B and Figure S1). While no differences were observed in lamellipodia protrusion rates in any of the assayed cellular conditions (Figure 7A), cortactin knockdown (si) increased lamellipodia extension by an average of $5.8 \mu \mathrm{m}$ over the maximum extension length observed in control cells (Figure 7A and B). Lamellipodia formed in cortactin si cells failed to effectively retract lamellipodia, demonstrating a $\sim 2$-fold increase in average lamellipodia persistence over control levels (Figure 7A and Figure S2). These results are consistent with the observed increase in lamellipodia extension and persistence observed when MTLn3 cells contact EGFcoated bead matrices [23]. These effects are fully rescued to control levels upon expression of WT GFP-cortactin (si+WT; Figure 7A and B). Although expression of GFP-cortactin S405/418A in cortactin si cells did not impact EGF-induced lamellipodia extension, average lamellipodia persistence was reduced by $46 \%$, from $195 \mathrm{sec}$ in $\mathrm{si}+\mathrm{WT}$ cells to $106 \mathrm{sec}$ in si+405,418 cells (Figure 7A). The lamellipodia in si+405,418 cells displayed series of multiple short extensions and retractions, had enhanced ruffling 
and appeared more labile than control or si+WT cells (Figure 7B: Figures S1 and S2 compared to Figure S4). These results suggest that S405/418 phosphorylation is vital in regulating lamellipodia actin dynamics responsible for proper protrusive behavior. 


\section{Discussion}

While the effects of cortactin phosphorylation at S405 and S418 by ERK1/2 have been studied at the biochemical and functional level in several systems [29,45,46,47], the spatial and temporal evaluation of S405 and $\$ 418$ phosphorylation have been hampered due to the lack of suitable reagents to directly study these sites in cellular and tissue contexts. Our development of anti-pS405 and anti-p418 cortactin antibodies has allowed us to examine the localization and signaling pathways regulating these cortactin phosphorylation events. These antibodies, coupled with the use of phosphorylation-null mutant constructs, allowed us to validate and extend previous findings implicating these sites in the regulation of carcinoma cell motility and associated lamellipodia dynamics.

The localization of pS418 cortactin in carcinoma lamellipodia and invadopodia is consistent with the defined and emerging roles cortactin plays in regulating actin dynamics within these structures [26,62]. To date, all studies designed to evaluate the cellular effects of pS405/418 phosphorylation have relied on the use of phosphorylation null or phosphomimetic (S405/418D) constructs. In pancreatic tumor cells, S405/418A and S405/418D both promote lamellipodia protrusion over control levels, whereas S405/418A inhibits and S405/418D promotes cell motility [47]. While the ability of S405/418A to promote lamellipodia protrusion in these studies is unclear, the remaining results are consistent with an activating role for $\$ 405 / 418$ phosphorylation in lamellipodia dynamics and motility. Similar results were obtained in the analysis of S405/418 on invadopodia function, with S405/418A expression impairing and S405/418D promoting ECM degradation activity [29]. Phosphorylation of cortactin S418 within lamellipodia and invadopodia (Figure 1B) supports these results. Precisely where

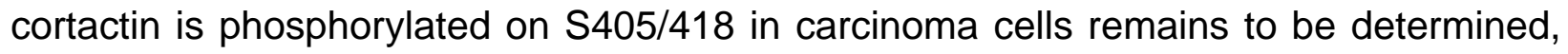
although the phosphorylating kinases ERK1/2 and PAK1 have been localized within lamellipodia $[63,64]$ and invadopodia [65]. This could suggest that cortactin is initially localized to lamellipodia or invadopodia, where it is subsequently phosphorylated on S405/418 when associated with the cortical actin networks within these structures. An 
analogous mechanism is employed for cortactin tyrosine phosphorylation within lamellipodia [40].

In HNSCC and several other tumor types, cortactin is present in the cytoplasm and is enriched at cell-cell junctions $[41,66,67]$. The localization of pS418 cortactin at regions of HNSCC cellular contact within tumors resembles the localization pattern of pY421 cortactin in this tumor type [68]. The staining pattern of cortactin and its tyrosine phosphorylated form is reminiscent of that found in two-dimensional epithelial monolayers, where cortactin has been shown to be essential for Arp2/3-mediated actin remodeling resultant from E-cadherin homoligation and subsequent Src activity $[69,70]$. While the presence of pS418 cortactin at these sites suggests additional functional roles for cortactin in E-cadherin-mediated actin regulation within tumors, whether or not cortactin S405/418 phosphorylation impacts elements of E-cadherin-based regulation of solid tumor behavior (such as tumor cell cohesion, motility or dissemination) remains to be examined.

Selumetinib inhibition of cortactin S405/S418 phosphorylation is consistent with results obtained with non-clinical MEK inhibitors [29,45], reinforcing the MEK-ERK1/2 pathway as the main signaling route responsible for phosphorylating these cortactin sites in tumor cells. This is supported by direct phosphorylation of cortactin by ERK1/2 in vitro [45] along with our data demonstrating concomitant downregulation of active ERK1/2 resultant of selumetinib treatment. In addition to MEK, PAK1 has recently been shown to phosphorylate cortactin at S405/418, regulating N-WASp actin dynamics responsible for clathrin- and caveolin-independent endocytosis [48]. PAK1 is activated primarily by binding to active Cdc42 or Rac1 [71], although alternative modes of activation have also been described [72]. Activated PAK1 also binds and activates MEK, stimulating ERK1/2 activation [73]. Since MEK inhibition largely ablates $S 405 / 418$ phosphorylation

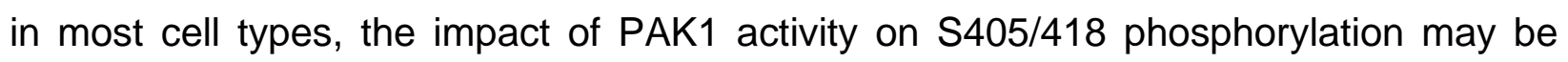
context dependent, with direct PAK1 phosphorylation of cortactin S405/418 regulating actin polymerization required for endosomal trafficking, while MEK-mediated phosphorylation (activated by Raf or other MEK activators) may be primarily responsible for governing motility-based actin dynamics. In addition, the related kinase PAK3 
phosphorylates cortactin at S113, an event that downregulates the ability of cortactin to bind F-actin and is important in modulating invadopodia function [29,74]. While our understanding regarding the interrelationship and regulation between PAK and MEK in governing cortactin S405/418 phosphorylation is currently incomplete, it is clear that the PAK-MEK-ERK1/2 signaling nexus impinges at multiple levels on cortactin to regulate actin dynamics involved in several membrane-based cellular processes.

Consistent with other reports [44,45], we observed the MEK-dependent EGF-induced shifting of cortactin from the $80 \mathrm{kDa}$ to $85 \mathrm{kDa}$ form by Western blotting. Direct analysis with anti-pS405 and anti-pS418 antibodies indicates that the $85 \mathrm{kDa}$ form is almost exclusively phosphorylated on these residues, as was determined by ${ }^{32} \mathrm{P}$ labeling and tryptic peptide analysis [44,45]. The shift in cortactin $M_{\mathrm{r}}$ is not attributable to bulk addition of phosphate, since phosphatase treatment of cortactin immunoprecipitates from EGF-treated cells failed to reconvert the $85 \mathrm{kDa}$ form to $80 \mathrm{kDa}$ (data not shown). While the distinct $80 \mathrm{kDa}$ and $85 \mathrm{kDa}$ bands represent different post-translationally modified cortactin forms associated with pS405/418 phosphorylation, mutations at these sites have no effect on $80 / 85 \mathrm{kDa}$ cortactin ratios, with the S405/418A mutant displaying a similar cortactin electrophoretic pattern to wild type cortactin (Figure 4). This suggests that $S 405 / 418$ cortactin phosphorylation, while associated with the shift from 80 to $85 \mathrm{kDa}$, is not necessary for generation of the $85 \mathrm{kDa}$ cortactin form. This is supported by the presence of $80 \mathrm{kDa}$ and $85 \mathrm{kDa}$ cortactin forms produced in kinase-free systems $[30,75]$ and by the existence of a single $85 \mathrm{kDa}$ form when analyzed by urea denaturing SDS-PAGE [75].

The lack of detailed structural data for cortactin derived by nuclear magnetic resonance spectroscopy or X-ray crystallography has hindered the field in understanding conformational changes cortactin undertakes in response to post-translational modifying events. The existence of cortactin in a "closed" versus "open" form regulated by S405/418 phosphorylation has been proposed to explain the observed 80 to $85 \mathrm{kDa}$ shift [45]. Support for this is derived from biochemical studies on N-WASp activation by the cortactin SH3 domain, where S405/418 phosphorylation enhances N-WASp activation and Arp2/3 actin nucleation activity [46]. These studies propose that the "closed" 
cortactin form undergoes an autoinhibitory conformational state where the carboxyl terminal helical proline-rich (HP) domain containing S405 and S418 is altered to render the SH3 domain inaccessible to binding N-WASp or other proteins. Phosphorylation of S405/418 results in liberating the SH3 domain, where it in turn is capable of binding and stimulating N-WASp activation. Expression of cortactin S405/418D phosphomimetic forms in cells increases branched actin networks in actin tails associated with cytoplasmic vesicles, providing support for this model in promoting cellular actin polymerization [47]. Initial assessments of cortactin structure by rotary shadow electron microscopy revealed cortactin to exist as a rod shaped monomer $220 \AA$ in length [76]. However, a recent biophysical analysis utilizing chemical crosslinking and small angle $x$ ray scattering suggests that cortactin exists in a more globular form, with the carboxyl terminal HP and $\mathrm{SH} 3$ domains folding back onto the amino terminal actin binding region [77]. Such a structure would support a "closed" conformation, although "open" structures were not observed, nor were the effects of ERK1/2 phosphorylation evaluated. Additional evidence for an inhibitory function of the amino terminus can be inferred from the ability of the cortactin carboxyl terminal domain to promote N-WASPdependent cell motility as effectively as wild type cortactin [78], as well as the prevalence of the $85 \mathrm{kDa}$ form in invasive colorectal cancer [66].

In the ERK-Src "switch" model proposed for cortactin regulation, cortactin phosphorylation by Src at Y421, 470 and 486 serves to downregulate N-WASp activity promoted by $\mathrm{S} 405 / 418$ phosphorylation [46]. This model therefore suggests that serine and tyrosine phosphorylation of cortactin function in a reciprocal manner to govern $\mathrm{N}$ WASp activation [58]. Our data with site-specific phosphorylation antibodies on lysates from EGF-stimulated cells indicates that S405/418 and Y421 are co-phosphorylated, and analysis of point mutant cortactin constructs does not indicate a reciprocal influence between cortactin serine and tyrosine phosphorylation events. These data suggest that cortactin function is not exclusively regulated by a serine-tyrosine "switch" mechanism. This view is additionally reinforced by the presence of pS418 and pY421 cortactin within lamellipodia and invadopodia. While our data do not rule out scenarios where such a mechanism may be employed at the cellular level, they are consistent with biochemical and cellular evidence indicating that tyrosine phosphorylation promotes N-WASp activity 
through binding of the adaptor Nck1 [37,39], a component that was not present in the original assays where the "switch" mechanism was defined. The ability of cortactin to be simultaneously phosphorylated at $\mathrm{S} 405 / 418$ and $\mathrm{Y} 421 / 470 / 486$ may therefore provide cells with the ability to fine-tune the level of N-WASp activation and subsequent actin remodeling in response to diverse upstream stimulatory input that triggers motility and invasion.

Consistent with the mechanistic descriptions above, inhibition of carcinoma cell motility by MEK inhibition and S405/418A expression indicates that S405/418 cortactin phosphorylation is important in promoting and maintaining cell migration. While similar results were observed in wound healing assays with pancreatic cancer cells [47], our work extends these findings by evaluating the effects of pS405/418 on lamellipodia dynamics. The inability of MTLn3 cells expressing S405/418A cortactin to maintain EGF-stimulated dominant lamellipodia persistence implies that the actin networks within these cells fail to maintain proper Arp2/3 nucleation, or are more labile following lamellipodia extension. While N-WASp activation and Arp2/3-mediated actin polymerization resultant of cortactin $\mathrm{SH} 3$ domain binding has been shown to be important in governing motility in multiple cell types [47,78], a detailed study of EGFinduced lamellipodia protrusion in this cell type has recently shown that WAVE2 and formin proteins, not N-WASp, are responsible for lamellipodia protrusion [14]. These results would therefore rule out a role for direct N-WASp activation by pS405/418 cortactin in MTLn3 lamellipodia extension. In addition to N-WASp, the cortactin SH3 domain interacts with several other proteins that have the potential to directly or indirectly regulate lamellipodia actin dynamics (reviewed in [62]). In particular, cortactin binds and activates the $\mathrm{Dbl}$ family guanine nucleotide exchange factor faciogenital dysplasia protein 1 (FGD1) [79,80], a potent activator of Cdc42 [50]. Cdc42 activity is required for localization of WAVE2 and its activator IRSp53 to the cell membrane, where it mediates lamellipodia extension [81]. FGD1 also activates the MEK-ERK1/2 pathway [50], allowing the potential of a positive feedback loop in stimulating cortactin S405/418 phosphorylation through continuous cortactin SH3-mediated FGD1 activity. FGD1 binding represents just one possible cortactin $\mathrm{SH} 3$ domain ligand with the capability to influence WAVE2 localization and lamellipodia dynamics. Whether such an 
FGD1-based regulatory circuit or other modes of potential pS405/418 cortactin regulation of WAVE2 activity exist in MTLn3 cells remains to be confirmed.

Previous studies on lamellipodia dynamics in other cells types indicate that cortactin removal decreases lamellipodia persistence, which can be rescued by re-expression of a cortactin amino terminal fragment lacking the carboxyl terminal region [20], eliminating contributions from pS405/418 in this system. These results differ from our work in MTLn3 cells, where cortactin removal results in enhanced persistence that can be rescued by re-expression of wild type cortactin. It is likely that these observed differences are due to a combination of different cell types, chemotactic cues, and analysis of dominant, initial lamellipodia versus steady-state lamellipodia dynamics [62]. Interestingly, inhibition of ERK1/2 signaling during macrophage lamellipodia extension results in decreased lamellipodia stability, with similar kymograph profiles to EGFstimulated MTLn3 cells with S405/418A expression [64]. These studies provide supporting evidence for our observations.

Through the use of phosphorylation-specific antibodies, we have analyzed the localization of cortactin pS405 and pS418 in tumor cells and tissue, as well as the signaling pathways regulating pS405/418 phosphorylation. Through the use of these reagents, we have been able to validate and further clarify the role of pS405/418 in cortactin-based signaling. Our functional studies of carcinoma motility and lamellipodia dynamics with phosphorylation-null constructs have shed additional light on the role these phosphorylation events play in regulating lamellipodia function involved in tumor cell movement. 


\section{Acknowledgements}

We thank LA Lopez-Skinner for technical assistance with site-directed mutagenesis and migration assays, R. Mooney (University of Rochester) for the MTLn3 cell line, P Smith, T Green and P Elvin at AstraZeneca for selumetinib and saracatinib, P. Turner and K. Secrest of the West Virginia University Department of Pathology Tissue Bank for histochemistry services, and A. Kapus (University of Toronto) for sharing technical information. The contributions of the West Virginia University Microscope Imaging Facility (supported by NIH grant P20 RR016440), Mary Babb Randolph Cancer Center are also gratefully acknowledged. 


\section{Author Contributions}

Conceived and designed the experiments: LCK, KEH, AGA, SAW. Performed the experiments: LCK, KEH, AGA. Acquired and analyzed the data: LCK, KEH, AGA, KHM. Contributed reagents/materials/analysis tools: KHM, SAW. Wrote the paper: LCK, KEH, AGA, SAW. 


\section{$\underline{\text { References }}$}

1. Yilmaz M, Christofori G (2010) Mechanisms of Motility in Metastasizing Cells. Mol Cancer Res.

2. Yamaguchi $\mathrm{H}$, Condeelis $\mathrm{J}$ (2006) Regulation of the actin cytoskeleton in cancer cell migration and invasion. Biochim Biophys Acta.

3. Olson MF, Sahai E (2009) The actin cytoskeleton in cancer cell motility. Clin Exp Metastasis 26: 273-287.

4. Insall RH, Machesky LM (2009) Actin dynamics at the leading edge: from simple machinery to complex networks. Dev Cell 17: 310-322.

5. Machesky LM (2008) Lamellipodia and filopodia in metastasis and invasion. FEBS Lett 582: 2102-2111.

6. Small JV, Stradal T, Vignal E, Rottner K (2002) The lamellipodium: where motility begins. Trends Cell Biol 12: 112-120.

7. Le Clainche C, Carlier MF (2008) Regulation of actin assembly associated with protrusion and adhesion in cell migration. Physiol Rev 88: 489-513.

8. Pollard TD, Borisy GG (2003) Cellular motility driven by assembly and disassembly of actin filaments. Cell 112: 453-465.

9. Pollard TD (2007) Regulation of actin filament assembly by Arp2/3 complex and formins. Annu Rev Biophys Biomol Struct 36: 451-477.

10. Blanchoin L, Pollard TD, Mullins RD (2000) Interactions of ADF/cofilin, Arp2/3 complex, capping protein and profilin in remodeling of branched actin filament networks. Curr Biol 10: 1273-1282.

11. Bernstein BW, Bamburg JR (2010) ADF/Cofilin: a functional node in cell biology. Trends Cell Biol.

12. Takenawa T, Suetsugu S (2007) The WASP-WAVE protein network: connecting the membrane to the cytoskeleton. Nat Rev Mol Cell Biol 8: 37-48. 
13. Pollitt AY, Insall RH (2009) WASP and SCAR/WAVE proteins: the drivers of actin assembly. J Cell Sci 122: 2575-2578.

14. Sarmiento C, Wang W, Dovas A, Yamaguchi H, Sidani M, et al. (2008) WASP family members and formin proteins coordinate regulation of cell protrusions in carcinoma cells. J Cell Biol 180: 1245-1260.

15. Weed SA, Parsons JT (2001) Cortactin: coupling membrane dynamics to cortical actin assembly. Oncogene 20: 6418-6434.

16. Daly RJ (2004) Cortactin signalling and dynamic actin networks. Biochem J 382: 1325.

17. Weed SA, Karginov AV, Schafer DA, Weaver AM, Kinley AW, et al. (2000) Cortactin localization to sites of actin assembly in lamellipodia requires interactions with $\mathrm{F}$ actin and the Arp2/3 complex. J Cell Biol 151: 29-40.

18. Uruno T, Liu J, Zhang P, Fan Y, Egile C, et al. (2001) Activation of Arp2/3 complexmediated actin polymerization by cortactin. Nat Cell Biol 3: 259-266.

19. Weaver AM, Karginov AV, Kinley AW, Weed SA, Li Y, et al. (2001) Cortactin promotes and stabilizes Arp2/3-induced actin filament network formation. Curr Biol 11: 370-374.

20. Bryce NS, Clark ES, Leysath JL, Currie JD, Webb DJ, et al. (2005) Cortactin promotes cell motility by enhancing lamellipodial persistence. Curr Biol 15: 12761285.

21. Boguslavsky S, Grosheva I, Landau E, Shtutman M, Cohen M, et al. (2007) p120 catenin regulates lamellipodial dynamics and cell adhesion in cooperation with cortactin. Proc Natl Acad Sci U S A 104: 10882-10887.

22. Gallet C, Rosa JP, Habib A, Lebret M, Levy-Toledano S, et al. (1999) Tyrosine phosphorylation of cortactin associated with Syk accompanies thromboxane analogue-induced platelet shape change. J Biol Chem 274: 23610-23616.

23. Kempiak SJ, Yamaguchi H, Sarmiento C, Sidani M, Ghosh M, et al. (2005) A neural Wiskott-Aldrich Syndrome protein-mediated pathway for localized activation of actin polymerization that is regulated by cortactin. J Biol Chem 280: 5836-5842. 
24. Lai FP, Szczodrak M, Oelkers JM, Ladwein M, Acconcia F, et al. (2009) Cortactin Promotes Migration and PDGF-induced Actin Reorganization by Signaling to Rho-GTPases. Mol Biol Cell.

25. Cosen-Binker LI, Kapus A (2006) Cortactin: the gray eminence of the cytoskeleton. Physiology (Bethesda) 21: 352-361.

26. Weaver AM (2008) Cortactin in tumor invasiveness. Cancer Lett 265: 157-166.

27. Artym VV, Zhang Y, Seillier-Moiseiwitsch F, Yamada KM, Mueller SC (2006) Dynamic interactions of cortactin and membrane type 1 matrix metalloproteinase at invadopodia: defining the stages of invadopodia formation and function. Cancer Res 66: 3034-3043.

28. Clark ES, Whigham AS, Yarbrough WG, Weaver AM (2007) Cortactin is an essential regulator of matrix metalloproteinase secretion and extracellular matrix degradation in invadopodia. Cancer Res 67: 4227-4235.

29. Ayala I, Baldassarre M, Giacchetti G, Caldieri G, Tete S, et al. (2008) Multiple regulatory inputs converge on cortactin to control invadopodia biogenesis and extracellular matrix degradation. J Cell Sci.

30. Wu H, Reynolds AB, Kanner SB, Vines RR, Parsons JT (1991) Identification and characterization of a novel cytoskeleton-associated pp60src substrate. Mol Cell Biol 11: 5113-5124.

31. Huang C, Liu J, Haudenschild CC, Zhan X (1998) The role of tyrosine phosphorylation of cortactin in the locomotion of endothelial cells. J Biol Chem 273: $25770-25776$.

32. Kapus A, Di Ciano C, Sun J, Zhan X, Kim L, et al. (2000) Cell volume-dependent phosphorylation of proteins of the cortical cytoskeleton and cell-cell contact sites. The role of Fyn and FER kinases. J Biol Chem 275: 32289-32298.

33. Crostella L, Lidder S, Williams R, Skouteris GG (2001) Hepatocyte Growth Factor/scatter factor-induces phosphorylation of cortactin in A431 cells in a Src kinase-independent manner. Oncogene 20: 3735-3745.

34. Huang J, Asawa T, Takato T, Sakai R (2003) Cooperative roles of Fyn and cortactin in cell migration of metastatic murine melanoma. J Biol Chem 278: 48367-48376. 
35. Boyle SN, Michaud GA, Schweitzer B, Predki PF, Koleske AJ (2007) A critical role for cortactin phosphorylation by Abl-family kinases in PDGF-induced dorsal-wave formation. Curr Biol 17: 445-451. 169

36. Bougneres L, Girardin SE, Weed SA, Karginov AV, Olivo-Marin JC, et al. (2004) Cortactin and Crk cooperate to trigger actin polymerization during Shigella invasion of epithelial cells. J Cell Biol 166: 225-235.

37. Tehrani S, Tomasevic N, Weed S, Sakowicz R, Cooper JA (2007) Src phosphorylation of cortactin enhances actin assembly. Proc Natl Acad Sci U S A 104: 11933-11938.

38. Lapetina S, Mader CC, Machida K, Mayer BJ, Koleske AJ (2009) Arg interacts with cortactin to promote adhesion-dependent cell edge protrusion. J Cell Biol 185: 503-519.

39. Oser M, Yamaguchi H, Mader CC, Bravo-Cordero JJ, Arias M, et al. (2009) Cortactin regulates cofilin and N-WASp activities to control the stages of invadopodium assembly and maturation. J Cell Biol 186: 571-587.

40. Head JA, Jiang D, Li M, Zorn LJ, Schaefer EM, et al. (2003) Cortactin tyrosine phosphorylation requires Rac1 activity and association with the cortical actin cytoskeleton. Mol Biol Cell 14: 3216-3229.

41. Rothschild BL, Shim AH, Ammer AG, Kelley LC, Irby KB, et al. (2006) Cortactin overexpression regulates actin-related protein $2 / 3$ complex activity, motility, and invasion in carcinomas with chromosome 11 q13 amplification. Cancer Res 66: 8017-8025.

42. Li Y, Tondravi M, Liu J, Smith E, Haudenschild CC, et al. (2001) Cortactin potentiates bone metastasis of breast cancer cells. Cancer Res 61: 6906-6911.

43. Martin KH, Jeffery ED, Grigera PR, Shabanowitz J, Hunt DF, et al. (2006) Cortactin phosphorylation sites mapped by mass spectrometry. J Cell Sci 119: 2851-2853.

44. van Damme $H$, Brok $H$, Schuuring-Scholtes $E$, Schuuring $E$ (1997) The redistribution of cortactin into cell-matrix contact sites in human carcinoma cells with $11 \mathrm{q} 13$ amplification is associated with both overexpression and posttranslational modification. J Biol Chem 272: 7374-7380. 
45. Campbell DH, Sutherland RL, Daly RJ (1999) Signaling pathways and structural domains required for phosphorylation of EMS1/cortactin. Cancer Res 59: 53765385 .

46. Martinez-Quiles N, Ho HY, Kirschner MW, Ramesh N, Geha RS (2004) Erk/Src phosphorylation of cortactin acts as a switch on-switch off mechanism that controls its ability to activate N-WASP. Mol Cell Biol 24: 5269-5280.

47. Kruchten AE, Krueger EW, Wang Y, McNiven MA (2008) Distinct phospho-forms of cortactin differentially regulate actin polymerization and focal adhesions. Am J Physiol Cell Physiol 295: C1113-1122.

48. Grassart A, Meas-Yedid V, Dufour A, Olivo-Marin JC, Dautry-Varsat A, et al. (2010) Pak1 phosphorylation enhances cortactin-N-WASP interaction in clathrincaveolin-independent endocytosis. Traffic.

49. Ammer AG, Kelley LC, Hayes KE, Evans JV, Lopez-Skinner LA, et al. (2009) Saracatinib impairs head and neck squamous cell carcinoma invasion by disrupting invadopodia function. J Cancer Sci Therapy 1: 052-061.

50. Olson MF, Pasteris NG, Gorski JL, Hall A (1996) Faciogenital dysplasia protein (FGD1) and Vav, two related proteins required for normal embryonic development, are upstream regulators of Rho GTPases. Curr Biol 6: 1628-1633.

51. Walker VG, Ammer A, Cao Z, Clump AC, Jiang BH, et al. (2007) PI3K activation is required for PMA-directed activation of cSrc by AFAP-110. Am J Physiol Cell Physiol 293: C119-132.

52. Sacks PG, Parnes SM, Gallick GE, Mansouri Z, Lichtner R, et al. (1988) Establishment and characterization of two new squamous cell carcinoma cell lines derived from tumors of the head and neck. Cancer Res 48: 2858-2866.

53. Krause CJ, Carey TE, Ott RW, Hurbis C, McClatchey KD, et al. (1981) Human squamous cell carcinoma. Establishment and characterization of new permanent cell lines. Arch Otolaryngol 107: 703-710.

54. Dry JR, Pavey S, Pratilas CA, Harbron C, Runswick S, et al. (2010) Transcriptional pathway signatures predict MEK addiction and response to selumetinib (AZD6244). Cancer Res 70: 2264-2273. 
55. Ohoka $Y$, Takai $Y(1998)$ Isolation and characterization of cortactin isoforms and a novel cortactin-binding protein, CBP90. Genes Cells 3: 603-612.

56. van Rossum AG, de Graaf JH, Schuuring-Scholtes E, Kluin PM, Fan YX, et al. (2003) Alternative splicing of the actin binding domain of human cortactin affects cell migration. J Biol Chem 278: 45672-45679.

57. Schuuring E, Verhoeven E, Litvinov S, Michalides RJ (1993) The product of the EMS1 gene, amplified and overexpressed in human carcinomas, is homologous to a v-src substrate and is located in cell-substratum contact sites. Mol Cell Biol 13: 2891-2898.

58. Lua BL, Low BC (2005) Cortactin phosphorylation as a switch for actin cytoskeletal network and cell dynamics control. FEBS Lett 579: 577-585.

59. Du Y, Weed SA, Xiong WC, Marshall TD, Parsons JT (1998) Identification of a novel cortactin SH3 domain-binding protein and its localization to growth cones of cultured neurons. Mol Cell Biol 18: 5838-5851.

60. Bailly M, Condeelis JS, Segall JE (1998) Chemoattractant-induced lamellipod extension. Microsc Res Tech 43: 433-443.

61. Segall JE, Tyerech S, Boselli L, Masseling S, Helft J, et al. (1996) EGF stimulates lamellipod extension in metastatic mammary adenocarcinoma cells by an actindependent mechanism. Clin Exp Metastasis 14: 61-72.

62. Ammer AG, Weed SA (2008) Cortactin branches out: roles in regulating protrusive actin dynamics. Cell Motil Cytoskeleton 65: 687-707.

63. Sells MA, Boyd JT, Chernoff $J$ (1999) p21-activated kinase 1 (Pak1) regulates cell motility in mammalian fibroblasts. J Cell Biol 145: 837-849.

64. Smith SD, Jaffer ZM, Chernoff J, Ridley AJ (2008) PAK1-mediated activation of ERK1/2 regulates lamellipodial dynamics. J Cell Sci 121: 3729-3736.

65. Furmaniak-Kazmierczak E, Crawley SW, Carter RL, Maurice DH, Cote GP (2007) Formation of extracellular matrix-digesting invadopodia by primary aortic smooth muscle cells. Circ Res 100: 1328-1336. 
66. Zhang LH, Tian B, Diao LR, Xiong YY, Tian SF, et al. (2006) Dominant expression of $85-\mathrm{kDa}$ form of cortactin in colorectal cancer. J Cancer Res Clin Oncol 132: 113-120.

67. Xu XZ, Garcia MV, Li TY, Khor LY, Gajapathy RS, et al. (2010) Cytoskeleton alterations in melanoma: aberrant expression of cortactin, an actin-binding adapter protein, correlates with melanocytic tumor progression. Mod Pathol 23: 187-196.

68. Ammer AG, Kelley LC, Hayes KE, Evans JV, Lopez-Skinner LA, et al. (2009) Saracatinib Impairs Head and Neck Squamous Cell Carcinoma Invasion by Disrupting Invadopodia Function. J Cancer Sci Ther 1: 52-61.

69. Helwani FM, Kovacs EM, Paterson AD, Verma S, Ali RG, et al. (2004) Cortactin is necessary for $\mathrm{E}$-cadherin-mediated contact formation and actin reorganization. $\mathrm{J}$ Cell Biol 164: 899-910.

70. Ren G, Helwani FM, Verma S, McLachlan RW, Weed SA, et al. (2009) Cortactin is a functional target of E-cadherin-activated Src family kinases in MCF7 epithelial monolayers. J Biol Chem 284: 18913-18922.

71. Manser E, Leung T, Salihuddin H, Zhao ZS, Lim L (1994) A brain serine/threonine protein kinase activated by Cdc42 and Rac1. Nature 367: 40-46.

72. Molli PR, Li DQ, Murray BW, Rayala SK, Kumar R (2009) PAK signaling in oncogenesis. Oncogene 28: 2545-2555.

73. Eblen ST, Slack JK, Weber MJ, Catling AD (2002) Rac-PAK signaling stimulates extracellular signal-regulated kinase (ERK) activation by regulating formation of MEK1-ERK complexes. Mol Cell Biol 22: 6023-6033.

74. Webb BA, Zhou S, Eves R, Shen L, Jia L, et al. (2006) Phosphorylation of cortactin by p21-activated kinase. Arch Biochem Biophys 456: 183-193.

75. Huang C, Ni Y, Wang T, Gao Y, Haudenschild CC, et al. (1997) Down-regulation of the filamentous actin cross-linking activity of cortactin by Src-mediated tyrosine phosphorylation. J Biol Chem 272: 13911-13915.

76. Weaver AM, Heuser JE, Karginov AV, Lee WL, Parsons JT, et al. (2002) Interaction of cortactin and N-WASp with Arp2/3 complex. Curr Biol 12: 1270-1278. 
77. Cowieson NP, King G, Cookson D, Ross I, Huber T, et al. (2008) Cortactin adopts a globular conformation and bundles actin into sheets. J Biol Chem 283: 1618716193.

78. Kowalski JR, Egile C, Gil S, Snapper SB, Li R, et al. (2005) Cortactin regulates cell migration through activation of N-WASP. J Cell Sci 118: 79-87.

79. Hou P, Estrada L, Kinley AW, Parsons JT, Vojtek AB, et al. (2003) Fgd1, the Cdc42 GEF responsible for Faciogenital Dysplasia, directly interacts with cortactin and mAbp1 to modulate cell shape. Hum Mol Genet 12: 1981-1993.

80. Kim K, Hou P, Gorski JL, Cooper JA (2004) Effect of Fgd1 on cortactin in Arp2/3 complex-mediated actin assembly. Biochemistry 43: 2422-2427.

81. El-Sibai M, Nalbant P, Pang H, Flinn RJ, Sarmiento C, et al. (2007) Cdc42 is required for EGF-stimulated protrusion and motility in MTLn3 carcinoma cells. J Cell Sci 120: 3465-3474. 


\section{Figure Legends}

Figure 1 Specificity and validation of pS405 and pS418 phospho-specific cortactin antibodies. (A) Phospho-specific recognition of anti-cortactin pS405 and pS418 antibodies. Clarified lysates $(50 \mu \mathrm{g})$ from 1483 cells transfected with Myc-tagged wildtype cortactin (WT), Myc-cortactin S405A, Myc-cortactin S418A or Myc-cortactin S405A,S418A point mutants were immunoblotted with affinity purified anti-Cort-pS418 (left) and anti-Cort-pS405 (right) antibodies. (B) Localization of pS418 cortactin in areas of motile and invasive actin dynamics. UMSCC2 cells (top row) were serum starved for $16 \mathrm{~h}$ prior to stimulation with $100 \mathrm{ng} / \mathrm{ml} \mathrm{EGF}$ for $1 \mathrm{~h}$ to induce lamellipodia formation, while UMSCC1 cells (middle row) were plated on FITC-conjugated gelatin coated coverslips (pseudocolored white) for $6 \mathrm{~h}$ to promote invadopodia formation. Cells were fixed, permeablized, and labeled with TRITC-phalloidin (Actin), anti-cortactin (Cort) and anti-cortactin-pS418 antibodies. Arrows denote localization of pS418 cortactin with total cortactin and F-actin in lamellipodia (top) and to invadopodia (middle) coinciding with areas of active matrix degradation. Bottom panels are magnified views of the indicated cellular region. Bars, $10 \mu \mathrm{m}$. (C) Localization of pS418 cortactin in HNSCC tumor tissue. Serial sections from a patient with invasive HNSCC were processed for immunohistochemistry with control IgG (Ctl), pS418 cortactin (pS418), total cortactin (Cort) and phospho-ERK1/2 (pERK) antibodies. Sections were counterstained with hematoxylin. Arrowheads indicate areas of peripheral pS418 cortactin and total cortactin enrichment. Bar, $100 \mu \mathrm{m}$.

Figure 2 Growth factor-stimulated Erk 1/2 activation mediates phosphorylation of cortactin at serine 405 and 418. (A) Growth factor-induced phosphorylation of cortactin S405 and S418. Serum starved UMSCC1 cells were stimulated with EGF (left) or FBS (right) for the indicated times. Cells were lysed and analyzed by Western blotting with anti-Cort-pS418 and anti-Cort-pS405 antibodies. Blots were stripped and reprobed with a pan-cortactin antibody to confirm equal loading (bottom). (B) Pharmacologic MEK inhibition inhibits cortactin S405 and S418 phosphorylation. UMSSC1 (left) and 1483 (right) cells were serum starved in the presence of the indicated selumetinib concentrations prior to stimulation with EGF for $20 \mathrm{~min}$. Cortactin immunoprecipitated 
from cell extracts was assayed by Western blotting with anti-Cort- pS418 and anti-CortpS405 antibodies. Blots were stripped and reprobed with pan- cortactin antibody as in (A) (bottom panels). Selumetinib efficacy was verified by the blotting of lyastes from selected timepoints with phospho-ERK1/2 (pERK1/2) and pan ERK1/2 antibodies (bottom). All blots are representative images from 3-4 independent experiments.

Figure 3 EGF-induced conversion of cortactin from $80 \mathrm{kDa}$ to $85 \mathrm{kDa}$ is impaired by Src and MEK1/2 inhibition. EGF induces the p80kDa to p85kDa shift in HNSCC cells. Serum starved UMSCC2 (A) and 1483 (B) cells were treated with 100ng/ml EGF for the indicated times. Clarified lysates were assayed by Western blotting with anti- cortactin, anti-Cort-pS418, anti-cort-pS405, anti-Cort-pY421, anti-Src-pY418, anti-pErk1/2 and total Erk1/2 antibodies as indicated. Red bars denote the position of the $85 \mathrm{kDa}$ cortactin form; black bars denote the $80 \mathrm{kDa}$ form. (C) Inhibition of Src and MEK1/2 kinase activity inhibits the cortactin "shift". UMSCC2 cells were treated with vehicle (DMSO), saracatinib, or selumetinib for $16 \mathrm{~h}$ in serum free media. Cells were stimulated with $100 \mathrm{ng} / \mathrm{ml}$ EGF for $1 \mathrm{~h}$, lysed and analyzed by Western blot analysis with an anticortactin antibody.

Figure 4 Cortactin tyrosine and serine phosphorylation resultant of v-Src activation are not interdependent. (A) Schematic diagram of the cortactin point mutant constructs assayed for phosphorylation. Mutated codons are denoted on the left and displayed with the corresponding mutant amino acid at the appropriate position within cortactin in red. (B) Murine fibroblasts lacking endogenous Src, Yes and Fyn (SYF) were transfected with murine-specific cortactin siRNA and cultured for $48 \mathrm{~h}$ to deplete endogenous cortactin. Cells were subsequently co-transected with the temperaturesensitive v-Src construct La29 (tsLa29) and wild-type or the indicated myc- tagged human cortactin point-mutant constructs at $41^{\circ} \mathrm{C}$ (non-permissive temperature). TPM; triple point mutant consisting of $\mathrm{Y}-\mathrm{F}$ mutations at positions 421,470 and 486 . After transfection, cells were cultured at $41^{\circ} \mathrm{C}$, then shifted to $35^{\circ} \mathrm{C}$ (permissive temperature) for $2 \mathrm{~h}$ to promote $\mathrm{v}$-Src activation. Recombinant cortactin proteins were assayed by immunoblotting with anti-cortactin-pY421, anti-cortactin-pS405, anti-myc, anti-cortactin, and anti- $\beta$-actin antibodies. Note that the inability of cortactin to be phosphorylated on 
Y421 does not impact its ability to be phosphorylated on S405, nor does lack of S405 phosphorylation impact Y421 phosphorylation.

Figure 5 Targeted inhibition of MEK1/2 inhibits HNSCC cell motility. 1483 and UMSCC1 cells $\left(5 \times 10^{5}\right)$ were starved for $24 \mathrm{~h}$ in the presence of vehicle (DMSO) or increasing concentrations of selumetinib as indicated. Cells were assayed for motility by electric substrate impedance sensing (ECIS) following stimulation with complete media containing the matched selumetinib concentration for $24 \mathrm{~h}$. Data is displayed as slope values calculated from the linear part of ECIS tracings. Bars represent mean \pm SE. *, $p<0.05$ compared to DMSO treated control cells.

Figure 6 Cortactin phosphorylation at serine 405 and 418 regulates carcinoma cell migration and adhesion. (A) Expression of GFP-cortactin constructs in MTLn3 cells. MTLn3 cells were transfected with murine-specific cortactin siRNA ( $\mathrm{Si}$ ) for $48 \mathrm{~h}$ to silence endogenous cortactin expression. Cells were subsequently transfected with the indicated human GFP-tagged cortactin wild-type and the various Erk1/2 phosphorylation-null point mutant constructs. Following transfection, cell lysates were immunoblotted with anti-cortactin, anti-GFP and anti- $\beta$-actin antibodies. Solid arrowheads indicate the position of GFP-tagged cortactin variants; open arrowheads denote the position of endogenous cortactin. (B) Serine 405 and 418 phosphorylation is required for efficient carcinoma cell motility. MTLn3 cells transfected as in (A) were analyzed for cell migration by ECIS. Cell impedance versus time plots for each transfected line are shown on the left; slope values calculated from the linear region of each plot are displayed on the right. (C) Carcinoma cell spreading requires phosphorylation of cortactin S405 and S418. Transfected MTLn3 cells were plated, with rates of spreading were monitored by ECIS tracings over time left. Slope values from the linear regions are shown on the right. Bars represent mean \pm SE for 3 independent experiments. ${ }^{*}, \mathrm{P}<0.05$ compared to control (ctl) cells.

Figure 7 Cortactin phosphorylation at serine 405 and 418 is required for lamellipodia persistence. (A) Kymographic analysis of MTLn3 lamellipodia. Serum starved MTLn3 cells (Ctl) or cells transfected with the indicated cortactin siRNA and cortactin constructs were monitored for dominant lamellipodia formation by live cell imaging following EGF 
stimulation. Quantification of lamellipodia protrusion rates, length of extension, and time of lamellipodia persistence are shown for each experimental condition. $\geq 10$ cells were analyzed for each group from $\geq 3$ independent experiments. (B) Representative kymograms of each cell type. Kymograms were constructed from 1-pixel wide lines drawn from the initial leading edge and in the direction of the dominant lamellipodia. Cells were visualized by fluorescent microscopy using mCherry- $\beta$-actin as the lamellipodia marker. Images were captured every $5 \mathrm{sec}$ for a period of $15 \mathrm{~min}$. Black lines denote the baseline position of the leading edge prior to EGF stimulation. Bar; 5 $\mu \mathrm{m}$. 


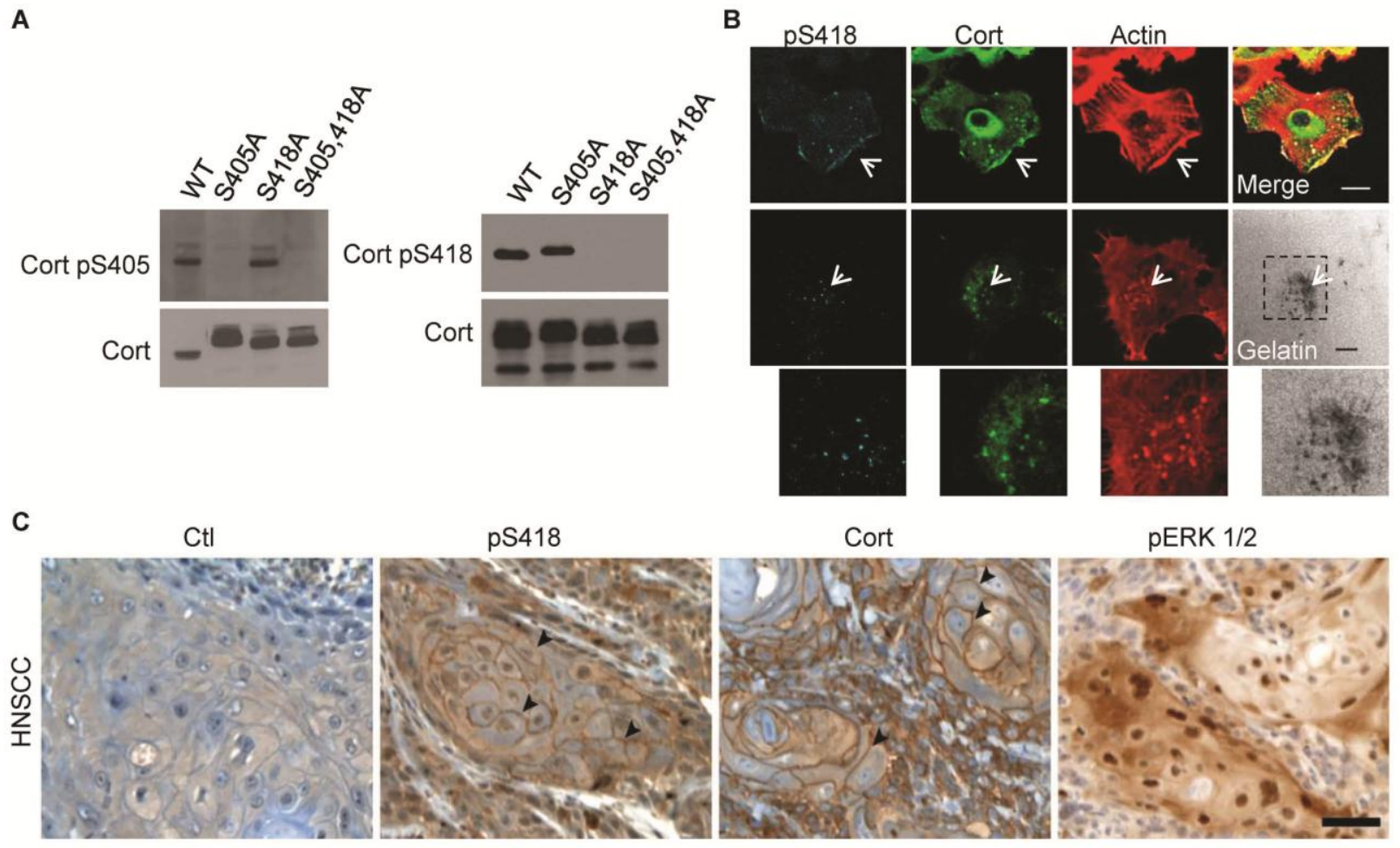

Kelley et al., Fig 1 
A

UMSCC 1

UMSCC1

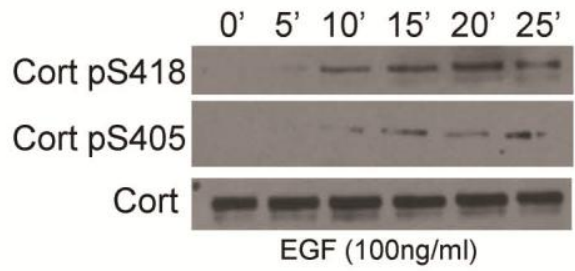

O' 5' $10^{\prime} \quad 15^{\prime} \quad 20^{\prime} \quad 25$

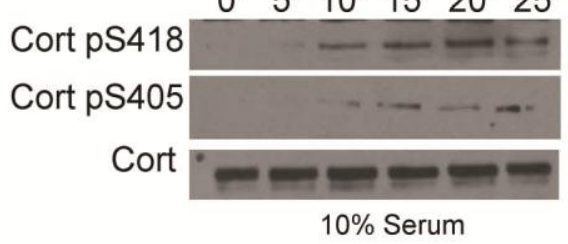

B
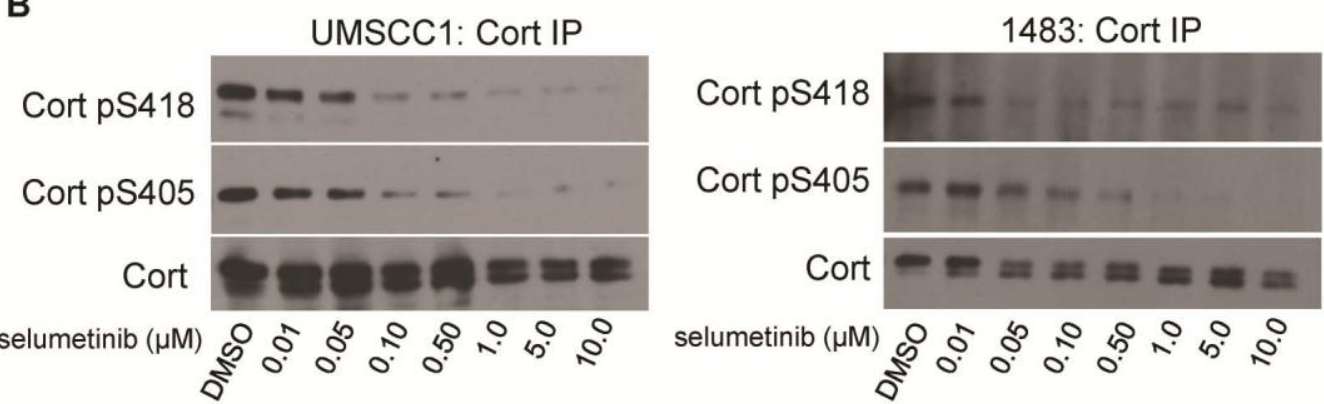

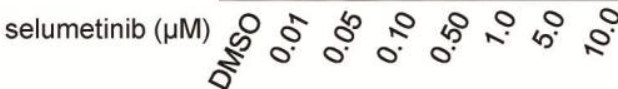

UMSCC1 : Input

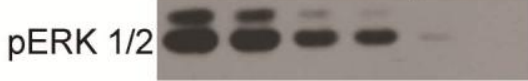

ERK 1/2

selumetinib $(\mu \mathrm{M}) \begin{gathered}5 \\ 5\end{gathered}$

1483: Input

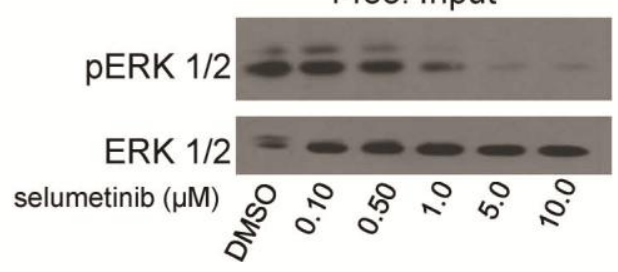

Kelley et al., Fig 2 


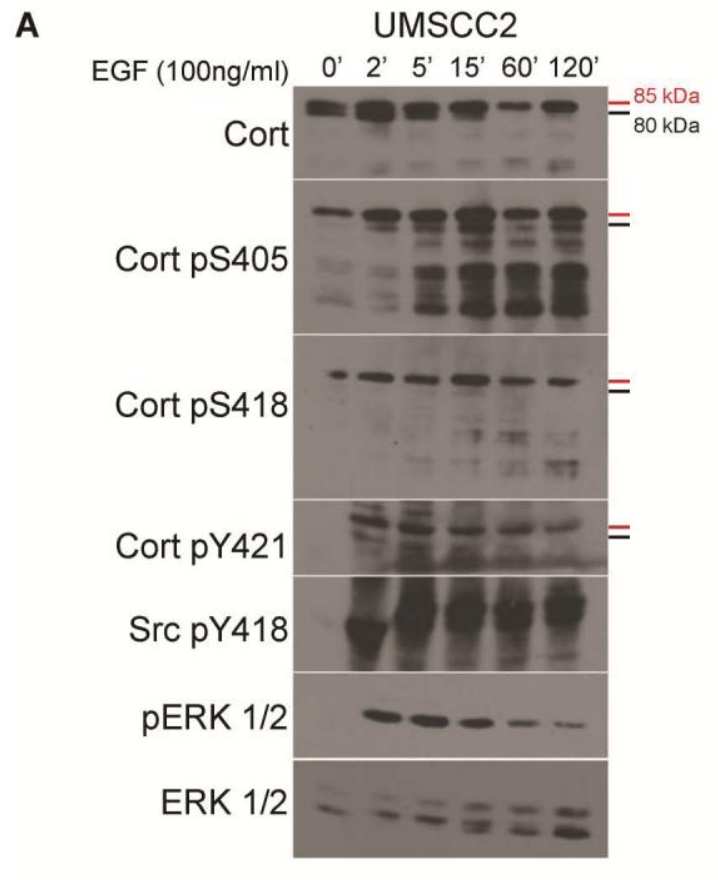

B 1483
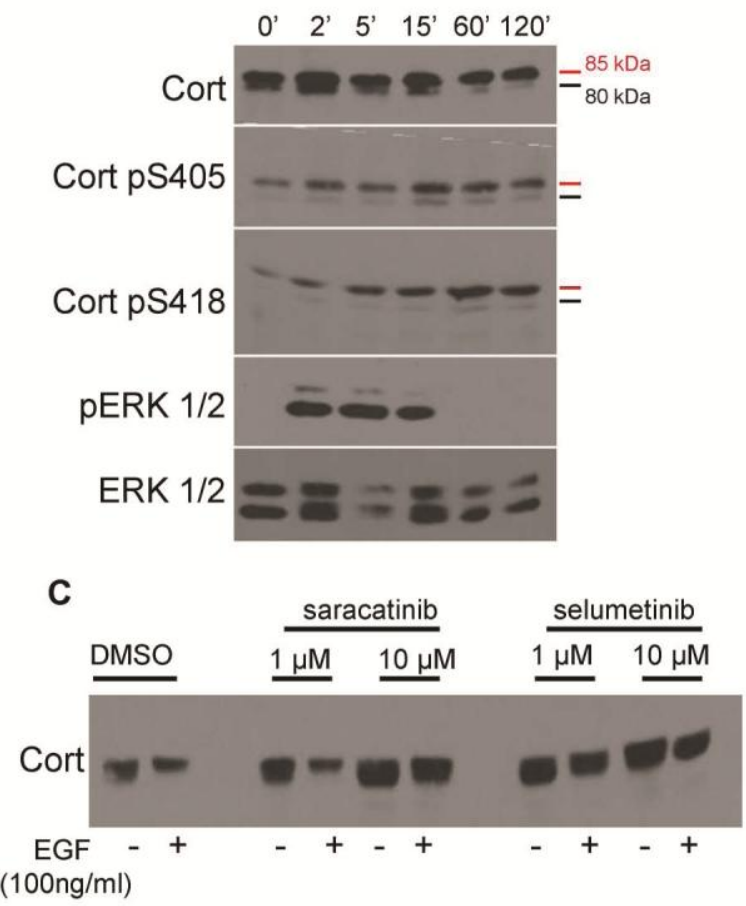

Kelley et al., Fig 3 
A

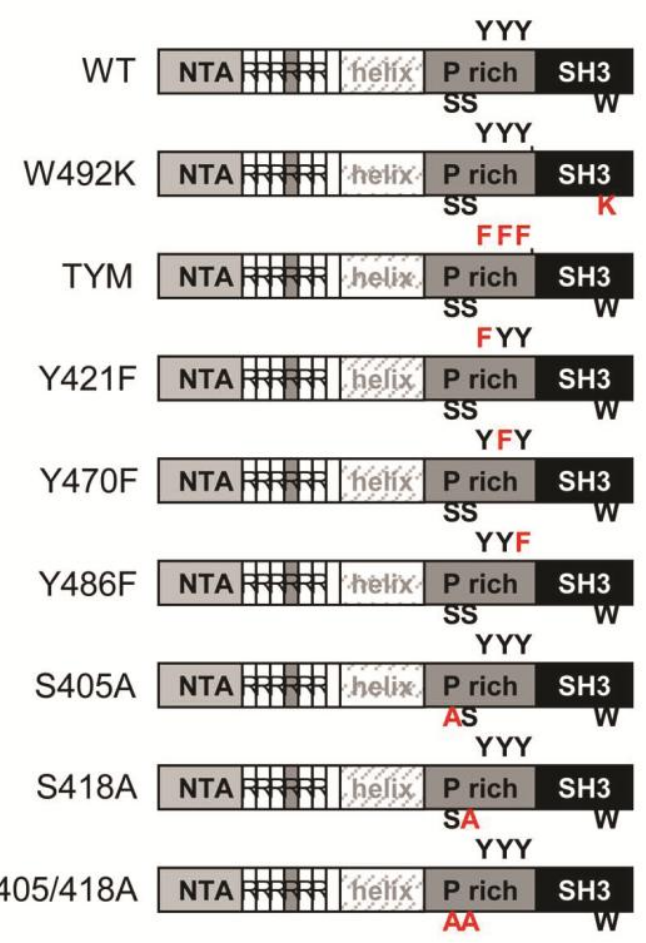

B

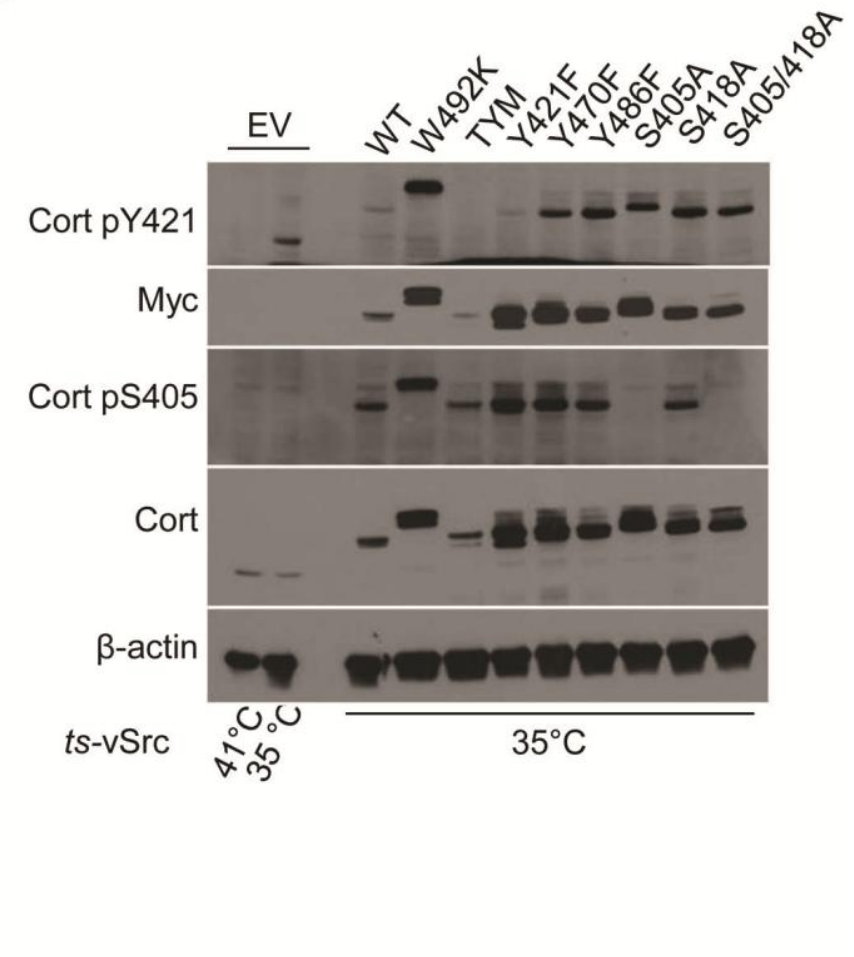

Kelley et al., Fig 4 

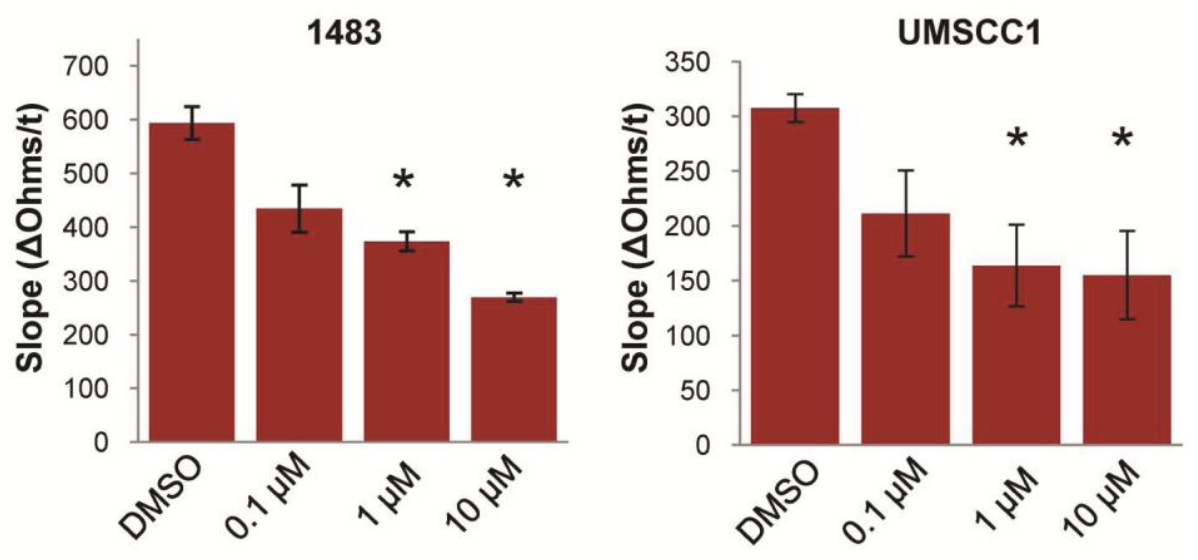

Kelley et al., Fig 5 


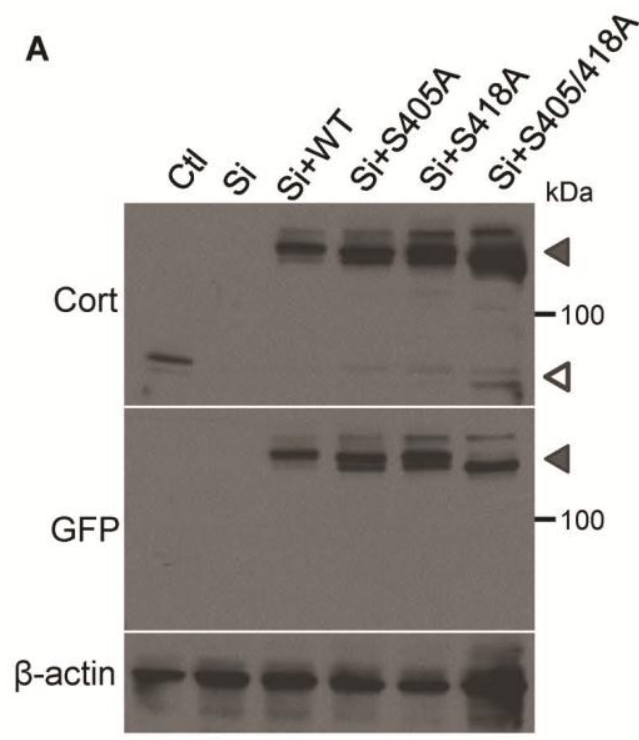

B
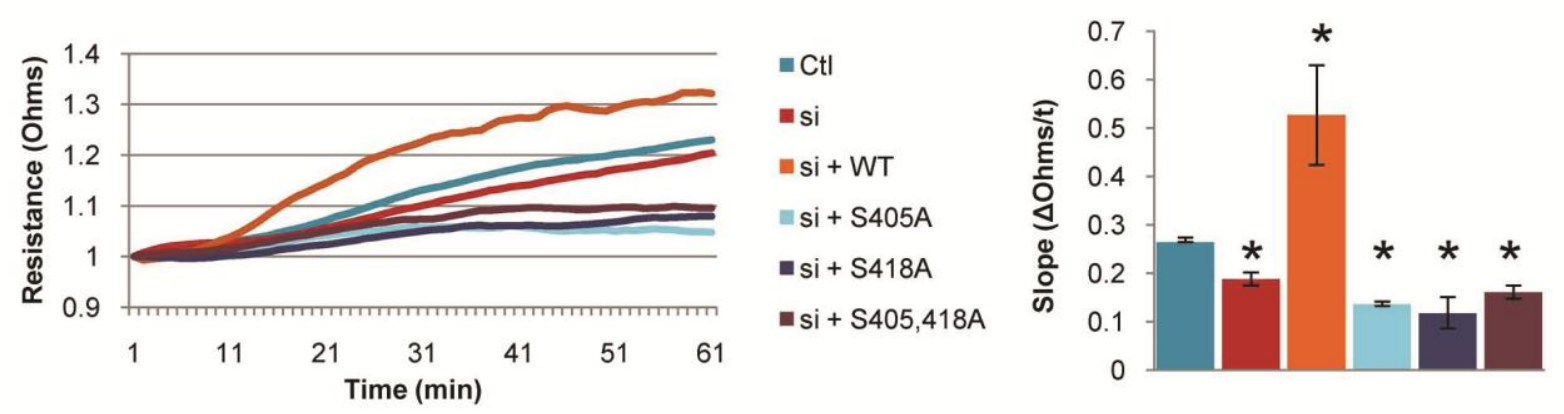

C

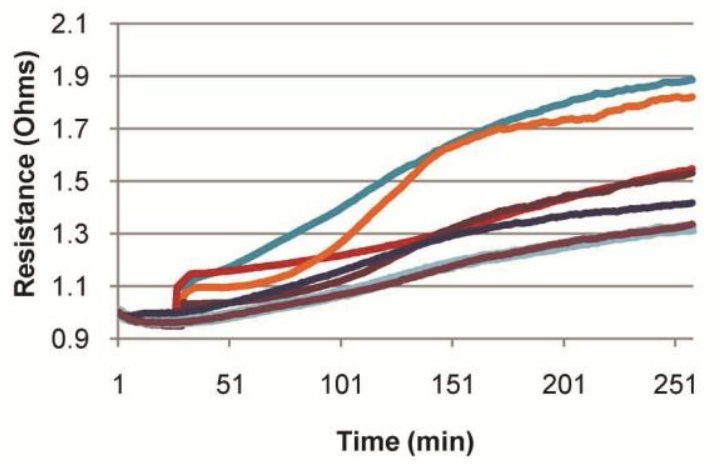

- Ctl

- si

vi + WT

si + S405A

= $\mathrm{si}+\mathrm{S} 418 \mathrm{~A}$

n $\mathrm{si}+\mathrm{S} 405,418 \mathrm{~A}$

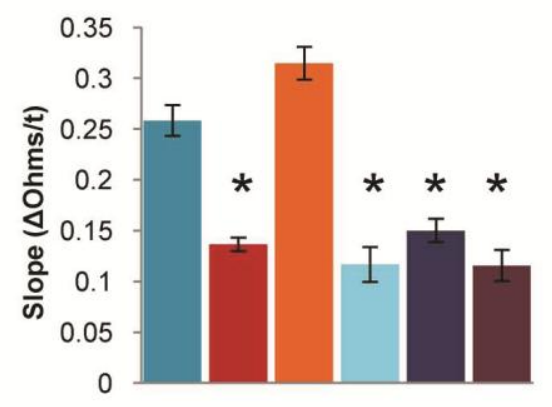

Kelley et al., Fig 6 
A
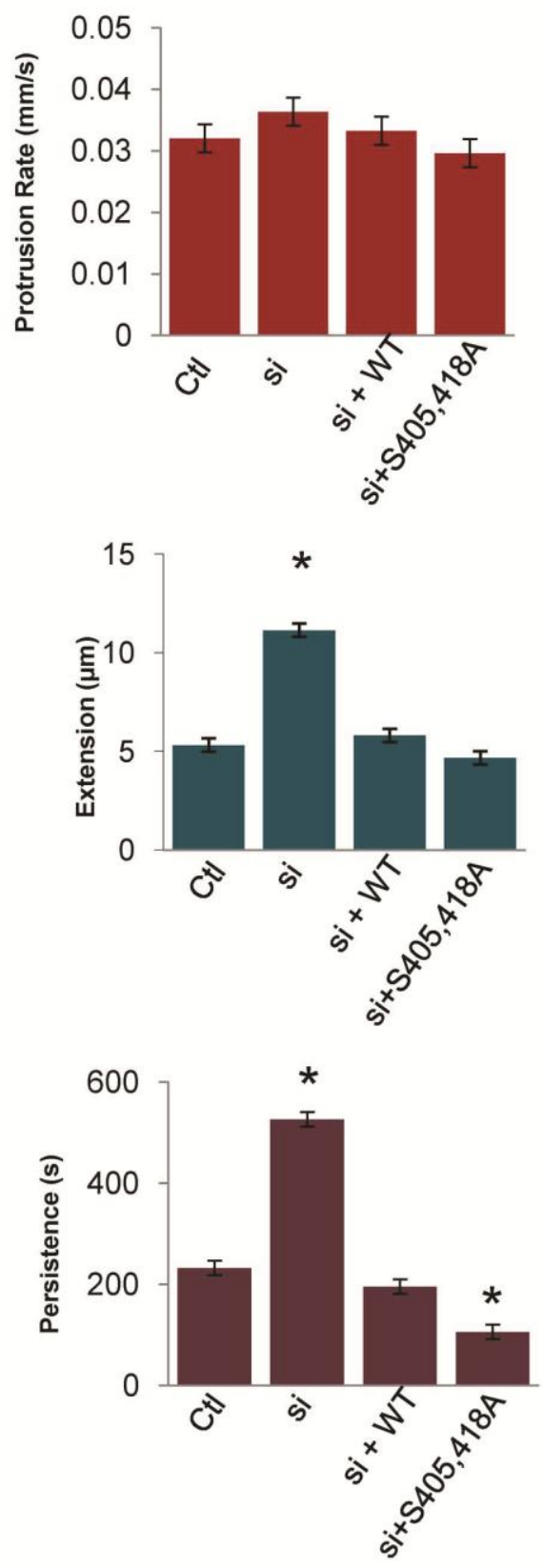

B

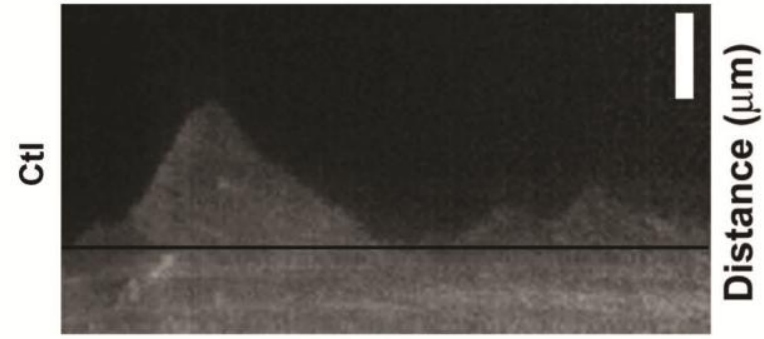

Time

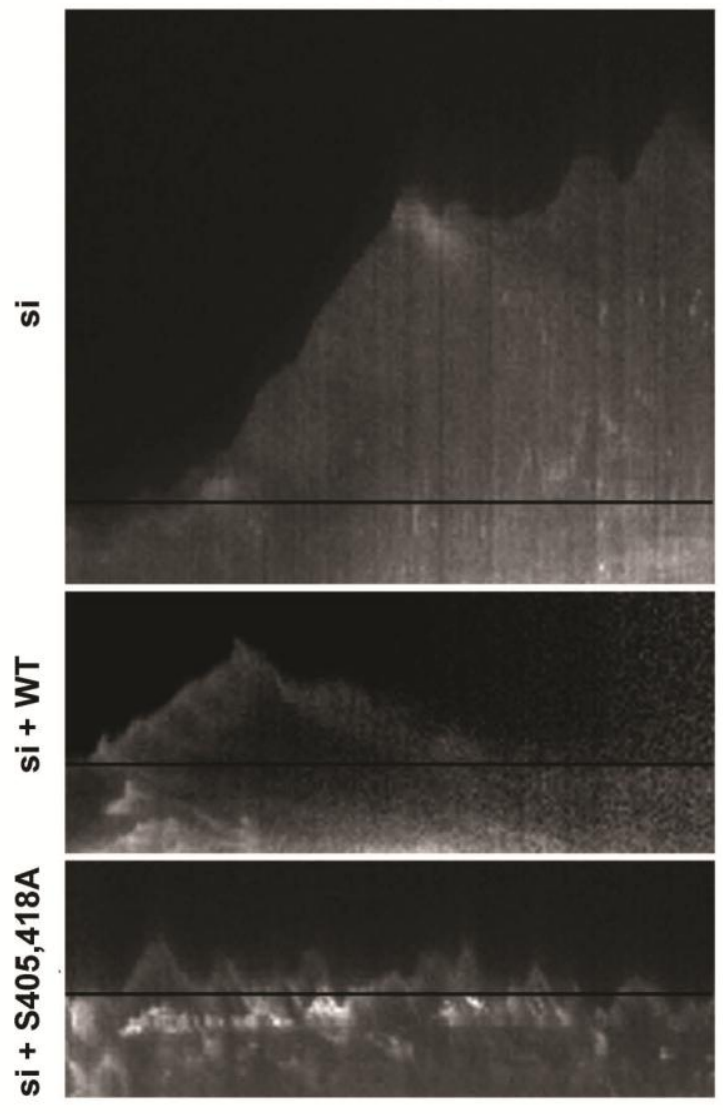

Kelley et al., Fig 7 


\section{General Discussion}

The successful treatment of CML and GIST patients with imatinib makes it an attractive chemotherapeutic agent for solid tumor types with elevated Abl activity (1-5). Unfortunately, single agent imatinib phase I and II trials have demonstrated little benefit for breast or pancreatic cancer patients. Furthermore, imatinib treatment of prostate, NSCLC, and HNSCC patients in clinical trials had deleterious effects, resulting in enhanced tumor progression (6-10,10-12). The poor response rate for chemotherapy targeting Abl in solid tumors may be attributed to the consequential oncogenic effects of Abl inhibition in these specific diseases.

On one hand, Abl family kinases enhance tumor proliferation and invasion in breast, melanoma, gastric and NSCLC (1,1-5,13-16). Conversely, Abl family kinases have also been shown to inhibit TGF $\beta$ induced EMT, tumor growth, and/or motility in breast cancer. Moreover, thyroid cancer cells treated with imatinib results in enhanced cell migration. In vivo, imatinib treatment increases breast cancer tumor size in xenograft mouse models, and; in vitro analysis of imatinib treated MA-11 breast cancer cells demonstrated enhanced migration, elevated Erk 1/2 and Wnt signaling (17-20). Study 1 provides additional insight into the divergent roles of $\mathrm{Abl}$ in tumor invasion by showing that $\mathrm{Abl}$ regulates invasive signaling in a tumor type specific manner (21). This work confirmed the published pro-invasive Abl family kinases activity in breast cancer invadopodia formation and 3-D collagen invasion utilizing the common MDA-MB-231 cell line $(4,5)$, as well as demonstrating an opposing role for Abl in HNSCC invasion where Abl functions an invasion suppressor.

Study 1 demonstrates elevated basal activation of Src and EGFR in HNSCC compared to MDA-MB-231 cells. Elevated Src activation in HNSCC can regulate ECM degradation and invasion by bypassing Abl family kinase regulation, contrary to the proposed EGFR/Src/Arg/Cortactin-mediated regulation of invadopodia maturation published by the Koleske group (4). In HNSCC, Src activity is responsible for the increased ECM-degradative activity of invadopodia induced by inhibition of Abl kinase activation by imatinib or RNAi. The regulatory role of Src in HNSCC was further examined in Study 2. The efficacy of the dual Src/Abl inhibitor saracatinib was 
demonstrated in HNSCC cell lines by Western blot analysis of Src substrate phosphorylation sites in FAK, p130Cas and cortactin. Study 2 confirmed the regulatory role of Src by the inhibition of invadopodia formation and activity, matrigel invasion and metastasis in vivo. Interestingly, analysis of CML cell lines resistant to the Abl-specific inhibitor nilotinib with overexpression of the Src-family member Lyn regained sensitivity to nilotinib when Lyn was removed by $\operatorname{RNAi}(22,23)$. Similar results were observed with imatinib-resistant human GIST xenograph models, where resistant tumors had integrinmediated elevated Src and Lyn activation (24). Collectively, these reports and the studies herein indicate that elevated Src kinase activity supersedes any pro- or antiinvasive role governed by Abl. In addition, these studies suggest the activation of Src resultant from Abl inhibition observed in Study 1 is not specific to HNSCC.

To determine the molecular mechanism for imatinib-mediated activation of Src in HNSCC and subsequent enhanced invadopodia proteolytic activity and invasion, we examined the activation of EGFR and Erk 1/2, two key components of invadopodia regulation. In Study 1, EGFR and Erk 1/2 activity were elevated in imatinib-treated HNSCC, but not in MDA-MB-231 cells. The increased Erk1/2 phosphorylation achieved with Abl family kinase inhibition is contrary to current dogma, where the RAS/RAF/MEK/Erk signaling pathway is activated by either BCR-Abl or integrinmediated c-Abl activity (25-27). Unexpectedly, increased phosphorylation of Erk $1 / 2$ is frequently observed in imatinib-resistant CML cells and other tumor types. CD34+,BCR$\mathrm{Abl}+$ progenitor leukemic cells treated with imatinib or nilotinib results in enhanced Erk 1/2 activation (28). Imatinib also stimulates elevated phosphorylation of Erk 1/2, PI3K/Akt, and Stat3 in PDGFR $\beta$ expressing glioblastoma cells (29). Conversely, imatinib treated colon adenocarcinoma cells display apoptosis and disrupted F-actin cytoskeletal networks (30). Such studies further highlight the need for better insight into the mechanisms utilized by tumors to acquire imatinib or nilotinib resistance if these compounds are to be considered for widespread use in solid tumors, as well as for current treatment of CML and GIST patients where imatinib and nilotinib forms an important part of the standard of care for these individuals. 
Along these lines, Packer et. al. have examined all known components of the RAS/RAF/MEK/Erk1/2 signaling pathway in an imatinib-resistant CML cell line, determining that imatinib induces activation of RAF and MEK in addition to Erk1/2. Cells lines from solid tumors were compared that contained constitutive inactivation of this pathway due to upstream KRAS mutation. When treated with imatinib, nilotinib or dasatinib, the RAF isoform BRAF was inhibited, but the related form CRAF was partially activated by heterodimerization with inactive BRAF, presenting a possible mechanism for imatinib activation of Erk1/2 (31). This is supported by the observation of CRAF hyperactivation in melanoma and thyroid cancer patients containing inactivating BRAF V600E mutations (32). MDA-MB-231 cells contain an activating KRAS mutation that hyperactivates KRAS/RAF/MEK/Erk1/2 and potentially diminishes the effect of imatinibinduced CRAF activation. This creates a plausible explanation for divergent effects observed with imatinib treatment between our HNSCC cell lines (UMSCC1 and OSC19) and MDA-MB-231. However, this mechanism does not explain the increased ECM degradation observed in UMSCC1 cells with Abl knocked-down seen in Study 1.

Elevated expression and secretion of HB-EGF in imatinib-treated HNSCC cell lines was shown by our laboratory (Study 1 ) and others (33), presenting an alternate mechanism for imatinib-mediated induction of EGFR, Src, and Erk 1/2 activity. Nevertheless, HBEGF is likely not the only cytokine up-regulated in HNSCC with imatinib treatment. Imatinib exposure enhanced ephrin and osteonectin in BCR-Abl+ leukemic cell lines $(34,35)$.

Cortactin is overexpressed in HNSCC and other cancers and correlates with poor prognosis (36-40). Elevated cortactin tyrosine phosphorylation is associated with increased invasion and metastasis (13,14,21,41-46). Cortactin is also phosphorylated by Erk $1 / 2$ at serine sites 405 and 418 , but little was known of the effects of cortactin serine phosphorylation on invasion and metastasis (47). Martinez-Quiles et al demonstrated cortactin serine phosphorylation increased N-WASp binding to the $\mathrm{SH} 3$ domain of cortactin, enhancing cortactin-mediated activation of Arp 2/3 actin polymerization (48). Study 3 further elucidated the effect of cortactin phosphorylation by Erk 1/2 on cancer cell motility and lamellipodia function. Serine 405/418 
phosphorylation resulted in increased adhesion and motility with in EGF-stimulated HNSCC cells. This motility enhancement can be attributed in part to an increase in lamellipodia persistence. Our laboratory (Study 3) and others have shown that cortactin serine phosphorylation and interaction with p120 catenin are essential for lamellipodia persistence and lamellipodial-mediated ECM recycling (49,50). Cortactin serine phosphorylation by Erk $1 / 2$ also modulates invadopodia formation and proteolytic activity (51). These results collectively support a role for Erk 1/2 phosphorylation of cortactin in regulating motility structures utilized for amoeboid, mesenchymal and collective cell motility and invasion (52).

Imatinib treatment enhances Erk 1/2, Src, and/or EGFR phosphorylation in HNSCC, $\mathrm{CML}$ and other tumor types. Our laboratory and others have demonstrated that imatinib can increase or decrease invasion and metastasis by activating or inhibiting key components of these processes- namely Erk1/2, Src, and EGFR. Abl appears to be a key component in mediating these divergent effects and can function as an anti- or prooncogenic protein dependent on cellular context. The regulating mechanism(s) that modulate the dichotomous roles of Abl in tumor motility are currently unknown. An understanding of this complex process is crucial as the use of imatinib expands to solid tumors in the clinic in order to prevent detrimental effects to patients undergoing imatinib treatment.

Imatinib is the first successful targeted kinase inhibitor that moved from the bench to the clinic, ushering in the era of modern targeted therapeutic intervention in cancer. The efficacy of these targeted therapies has been mixed, from the success of imatinib to the puzzling lack of response with dasatinib in phase II and III trials where preclinical work showed great promise. Why certain targets are more effective than others when therapeutically inhibited is not fully understood, nor are the compensatory mechanisms tumor cells utilize to overcome targeted therapies in developing drug resistance.

Drug resistance has been attributed to three main reasons; the heterogeneity of tumors that enable a subset of tumors cells (likely tumor initiating stem cells) to survive chemotherapeutic agents, the complexity of the microenvironment (cellular and noncellular) providing a protective niche for the tumor cells; and tumor cell plasticity (53). 
Tumor plasticity allows the tumor to reversibly change and adapt their phenotype to intrinsic and external cues without permanently altering genomic DNA. EMT confers stem cell like properties and chemoresistance in tumor cells. Transcription factors such as Zeb1, Twist, and Snail are key components that regulate EMT and are current areas of intense focus in efforts to reverse molecular and phenotypic elements of transformation (54). However, it is clear that EMT is not the sole factor regulating tumor plasticity (55), as our work and others indicates that adaptive kinase responses play important roles in mediating drug resistance $(21,28,29)$. Duncan et. al. utilized an innovative multiplexed inhibitor beads (MIBs) system that binds over $60 \%$ of the kinase kinome to examine the rapid kinome reprogramming induced by MEK inhibitor treatment of triple negative breast cancer (56). MIBs are generated by immobilizing several broad kinase inhibitors to sepharose beads. MIBs selectively bind active kinases due to the general mechanism utilized by kinase inhibitors, blocking the ATP binding sites; this allows the monitoring of altered activity of over a hundred kinases simultaneously (56). Our work demonstrates kinase "reprogramming" within hours after drug treatment enhances HNSCC metastasis and invasion making imatinib an interesting candidate for this process. The dichotomous functions of $\mathrm{Abl}$ in different cancer types suggest that it may play a key regulatory role in governing tumor plasticity responsible for facilitating drug resistance. 


\section{$\underline{\text { References }}$}

(1) Srinivasan D, Plattner R. Activation of Abl tyrosine kinases promotes invasion of aggressive breast cancer cells. Cancer Res 2006 Jun 1;66(11):5648-5655.

(2) Srinivasan D, Sims JT, Plattner R. Aggressive breast cancer cells are dependent on activated Abl kinases for proliferation, anchorage-independent growth and survival. Oncogene 2008 Feb 14;27(8):1095-1105.

(3) Ganguly SS, Fiore LS, Sims JT, Friend JW, Srinivasan D, Thacker MA, et al. c-Abl and Arg are activated in human primary melanomas, promote melanoma cell invasion via distinct pathways, and drive metastatic progression. Oncogene 2011 Sep 5.

(4) Mader CC, Oser M, Magalhaes MA, Bravo-Cordero JJ, Condeelis J, Koleske AJ, et al. An EGFR-Src-Arg-cortactin pathway mediates functional maturation of invadopodia and breast cancer cell invasion. Cancer Res 2011 Mar 1;71(5):1730-1741.

(5) Smith-Pearson PS, Greuber EK, Yogalingam G, Pendergast AM. Abl kinases are required for invadopodia formation and chemokine-induced invasion. J Biol Chem 2010 Dec 17;285(51):40201-40211.

(6) Tsao AS, Liu S, Fujimoto J, Wistuba II, Lee JJ, Marom EM, et al. Phase II trials of imatinib mesylate and docetaxel in patients with metastatic non-small cell lung cancer and head and neck squamous cell carcinoma. J Thorac Oncol 2011 Dec;6(12):21042111.

(7) Cristofanilli M, Morandi P, Krishnamurthy S, Reuben JM, Lee BN, Francis D, et al. Imatinib mesylate (Gleevec) in advanced breast cancer-expressing C-Kit or PDGFRbeta: clinical activity and biological correlations. Ann Oncol 2008 Oct;19(10):1713-1719.

(8) Modi S, Seidman AD, Dickler M, Moasser M, D'Andrea G, Moynahan ME, et al. A phase II trial of imatinib mesylate monotherapy in patients with metastatic breast cancer. Breast Cancer Res Treat 2005 Mar;90(2):157-163.

(9) Chew HK, Barlow WE, Albain K, Lew D, Gown A, Hayes DF, et al. A phase II study of imatinib mesylate and capecitabine in metastatic breast cancer: Southwest Oncology Group Study 0338. Clin Breast Cancer 2008 Dec;8(6):511-515.

(10) Lin AM, Rini BI, Weinberg V, Fong K, Ryan CJ, Rosenberg JE, et al. A phase II trial of imatinib mesylate in patients with biochemical relapse of prostate cancer after definitive local therapy. BJU Int 2006 Oct;98(4):763-769. 
(11) Gharibo M, Patrick-Miller L, Zheng L, Guensch L, Juvidian P, Poplin E. A phase II trial of imatinib mesylate in patients with metastatic pancreatic cancer. Pancreas 2008 May;36(4):341-345.

(12) Chen J, Rocken C, Nitsche B, Hosius C, Gschaidmeier H, Kahl S, et al. The tyrosine kinase inhibitor imatinib fails to inhibit pancreatic cancer progression. Cancer Lett 2006 Feb 28;233(2):328-337.

(13) Lapetina S, Mader CC, Machida K, Mayer BJ, Koleske AJ. Arg interacts with cortactin to promote adhesion-dependent cell edge protrusion. J Cell Biol 2009 May 4;185(3):503-519.

(14) Boyle SN, Michaud GA, Schweitzer B, Predki PF, Koleske AJ. A critical role for cortactin phosphorylation by Abl-family kinases in PDGF-induced dorsal-wave formation. Curr Biol 2007 Mar 6;17(5):445-451.

(15) Srinivasan D, Kaetzel DM, Plattner R. Reciprocal regulation of Abl and receptor tyrosine kinases. Cell Signal 2009 Jul;21(7):1143-1150.

(16) Lin J, Sun T, Ji L, Deng W, Roth J, Minna J, et al. Oncogenic activation of c-Abl in non-small cell lung cancer cells lacking FUS1 expression: inhibition of c-Abl by the tumor suppressor gene product Fus1. Oncogene 2007 Oct 25;26(49):6989-6996.

(17) Frasca F, Vigneri P, Vella V, Vigneri R, Wang JY. Tyrosine kinase inhibitor STI571 enhances thyroid cancer cell motile response to Hepatocyte Growth Factor. Oncogene 2001 Jun 28;20(29):3845-3856.

(18) Allington TM, Galliher-Beckley AJ, Schiemann WP. Activated Abl kinase inhibits oncogenic transforming growth factor-beta signaling and tumorigenesis in mammary tumors. FASEB J 2009 Dec;23(12):4231-4243.

(19) Noren NK, Foos G, Hauser CA, Pasquale EB. The EphB4 receptor suppresses breast cancer cell tumorigenicity through an Abl-Crk pathway. Nat Cell Biol 2006 Aug;8(8):815-825.

(20) Rappa G, Anzanello F, Lorico A. Imatinib mesylate enhances the malignant behavior of human breast carcinoma cells. Cancer Chemother Pharmacol 2011 Apr;67(4):919-926.

(21) Hayes KE, Walk EL, Ammer AG, Kelley LC, Martin KH, Weed SA. Ableson kinases negatively regulate invadopodia function and invasion in head and neck squamous cell carcinoma by inhibiting an HB-EGF autocrine loop. Oncogene 2012 Nov 12. 
(22) Mahon FX, Hayette S, Lagarde V, Belloc F, Turcq B, Nicolini F, et al. Evidence that resistance to nilotinib may be due to BCR-ABL, Pgp, or Src kinase overexpression. Cancer Res 2008 Dec 1;68(23):9809-9816.

(23) Gioia R, Leroy C, Drullion C, Lagarde V, Etienne G, Dulucq S, et al. Quantitative phosphoproteomics revealed interplay between Syk and Lyn in the resistance to nilotinib in chronic myeloid leukemia cells. Blood 2011 Aug 25;118(8):2211-2221 .

(24) Rossi F, Yozgat Y, de Stanchina E, Veach D, Clarkson B, Manova K, et al. Imatinib upregulates compensatory integrin signaling in a mouse model of gastrointestinal stromal tumor and is more effective when combined with dasatinib. Mol Cancer Res 2010 Sep;8(9):1271-1283.

(25) Renshaw MW, Lewis JM, Schwartz MA. The c-Abl tyrosine kinase contributes to the transient activation of MAP kinase in cells plated on fibronectin. Oncogene 2000 Jun 29;19(28):3216-3219.

(26) Renshaw MW, Lea-Chou E, Wang JY. Rac is required for v-Abl tyrosine kinase to activate mitogenesis. Curr Biol 1996 Jan 1;6(1):76-83.

(27) Goga A, McLaughlin J, Afar DE, Saffran DC, Witte ON. Alternative signals to RAS for hematopoietic transformation by the BCR-ABL oncogene. Cell 1995 Sep 22;82(6):981-988.

(28) Hartel N, Klag T, Hanfstein B, Mueller MC, Schenk T, Erben P, et al. Enhanced ABL-inhibitor-induced MAPK-activation in T315I-BCR-ABL-expressing cells: a potential mechanism of altered leukemogenicity. J Cancer Res Clin Oncol 2012 Feb;138(2):203212.

(29) Dong Y, Jia L, Wang X, Tan X, Xu J, Deng Z, et al. Selective inhibition of PDGFR by imatinib elicits the sustained activation of ERK and downstream receptor signaling in malignant glioma cells. Int J Oncol 2011 Feb;38(2):555-569.

(30) Popow-Wozniak A, Wozniakowska A, Kaczmarek L, Malicka-Blaszkiewicz M, Nowak D. Apoptotic effect of imatinib on human colon adenocarcinoma cells: influence on actin cytoskeleton organization and cell migration. Eur J Pharmacol 2011 Sep 30;667(1-3):66-73.

(31) Packer LM, Rana S, Hayward R, O'Hare T, Eide CA, Rebocho A, et al. Nilotinib and MEK inhibitors induce synthetic lethality through paradoxical activation of RAF in drug-resistant chronic myeloid leukemia. Cancer Cell 2011 Dec 13;20(6):715-727.

(32) Santarpia L, Lippman SM, El-Naggar AK. Targeting the MAPK-RAS-RAF signaling pathway in cancer therapy. Expert Opin Ther Targets 2012 Jan;16(1):103-119. 
(33) Johnson FM, Saigal B, Donato NJ. Induction of heparin-binding EGF-like growth factor and activation of EGF receptor in imatinib mesylate-treated squamous carcinoma cells. J Cell Physiol 2005 Nov;205(2):218-227.

(34) Suzuki M, Abe A, Imagama S, Nomura Y, Tanizaki R, Minami Y, et al. BCR-ABLindependent and RAS / MAPK pathway-dependent form of imatinib resistance in $\mathrm{Ph}$ positive acute lymphoblastic leukemia cell line with activation of EphB4. Eur J Haematol 2010 Mar;84(3):229-238.

(35) Fenouille N, Puissant A, Dufies M, Robert G, Jacquel A, Ohanna M, et al. Persistent activation of the Fyn/ERK kinase signaling axis mediates imatinib resistance in chronic myelogenous leukemia cells through upregulation of intracellular SPARC. Cancer Res 2010 Dec 1;70(23):9659-9670.

(36) Meredith SD, Levine PA, Burns JA, Gaffey MJ, Boyd JC, Weiss LM, et al. Chromosome 11q13 amplification in head and neck squamous cell carcinoma. Association with poor prognosis. Arch Otolaryngol Head Neck Surg 1995 Jul;121(7):790-794.

(37) Sugahara K, Michikawa Y, Ishikawa K, Shoji Y, Iwakawa M, Shibahara T, et al. Combination effects of distinct cores in 11 q13 amplification region on cervical lymph node metastasis of oral squamous cell carcinoma. Int J Oncol 2011 Oct;39(4):761-769.

(38) Buday L, Downward J. Roles of cortactin in tumor pathogenesis. Biochim Biophys Acta 2007 Jun;1775(2):263-273.

(39) Hui R, Ball JR, Macmillan RD, Kenny FS, Prall OW, Campbell DH, et al. EMS1 gene expression in primary breast cancer: relationship to cyclin D1 and oestrogen receptor expression and patient survival. Oncogene 1998 Aug 27;17(8):1053-1059.

(40) Li Y, Tondravi M, Liu J, Smith E, Haudenschild CC, Kaczmarek M, et al. Cortactin potentiates bone metastasis of breast cancer cells. Cancer Res 2001 Sep 15;61(18):6906-6911.

(41) Kelley LC, Ammer AG, Hayes KE, Martin KH, Machida K, Jia L, et al. Oncogenic Src requires a wild-type counterpart to regulate invadopodia maturation. J Cell Sci 2010 Nov 15;123(Pt 22):3923-3932.

(42) Oser M, Mader CC, Gil-Henn H, Magalhaes M, Bravo-Cordero JJ, Koleske AJ, et al. Specific tyrosine phosphorylation sites on cortactin regulate Nck1-dependent actin polymerization in invadopodia. J Cell Sci 2010 Nov 1;123(Pt 21):3662-3673. 
(43) Oser M, Yamaguchi H, Mader CC, Bravo-Cordero JJ, Arias M, Chen X, et al. Cortactin regulates cofilin and N-WASp activities to control the stages of invadopodium assembly and maturation. J Cell Biol 2009 Aug 24;186(4):571-587.

(44) Head JA, Jiang D, Li M, Zorn LJ, Schaefer EM, Parsons JT, et al. Cortactin tyrosine phosphorylation requires Rac1 activity and association with the cortical actin cytoskeleton. Mol Biol Cell 2003 Aug;14(8):3216-3229.

(45) Kruchten AE, Krueger EW, Wang Y, McNiven MA. Distinct phospho-forms of cortactin differentially regulate actin polymerization and focal adhesions. Am J Physiol Cell Physiol 2008 Nov;295(5):C1113-22.

(46) Kowalski JR, Egile C, Gil S, Snapper SB, Li R, Thomas SM. Cortactin regulates cell migration through activation of N-WASP. J Cell Sci 2005 Jan 1;118(Pt 1):79-87.

(47) Campbell DH, Sutherland RL, Daly RJ. Signaling pathways and structural domains required for phosphorylation of EMS1/cortactin. Cancer Res 1999 Oct 15;59(20):53765385 .

(48) Martinez-Quiles N, Ho HY, Kirschner MW, Ramesh N, Geha RS. Erk/Src phosphorylation of cortactin acts as a switch on-switch off mechanism that controls its ability to activate N-WASP. Mol Cell Biol 2004 Jun;24(12):5269-5280.

(49) Boguslavsky S, Grosheva I, Landau E, Shtutman M, Cohen M, Arnold K, et al. P120 Catenin Regulates Lamellipodial Dynamics and Cell Adhesion in Cooperation with Cortactin. Proc Natl Acad Sci U S A 2007 Jun 26;104(26):10882-10887.

(50) Kelley LC, Hayes KE, Ammer AG, Martin KH, Weed SA. Cortactin phosphorylated by ERK $1 / 2$ localizes to sites of dynamic actin regulation and is required for carcinoma lamellipodia persistence. PLoS One 2010 Nov 4;5(11):e13847.

(51) Ayala I, Baldassarre M, Giacchetti G, Caldieri G, Tete S, Luini A, et al. Multiple regulatory inputs converge on cortactin to control invadopodia biogenesis and extracellular matrix degradation. J Cell Sci 2008 Feb 1;121(Pt 3):369-378.

(52) Friedl P, Sahai E, Weiss S, Yamada KM. New dimensions in cell migration. Nat Rev Mol Cell Biol 2012 Nov;13(11):743-747.

(53) Saunders NA, Simpson F, Thompson EW, Hill MM, Endo-Munoz L, Leggatt G, et al. Role of intratumoural heterogeneity in cancer drug resistance: molecular and clinical perspectives. EMBO Mol Med 2012 Aug;4(8):675-684.

(54) Nieto MA. The ins and outs of the epithelial to mesenchymal transition in health and disease. Annu Rev Cell Dev Biol 2011 Nov 10;27:347-376. 
(55) Thompson EW, Haviv I. The social aspects of EMT-MET plasticity. Nat Med 2011 Sep 7;17(9):1048-1049.

(56) Duncan JS, Whittle MC, Nakamura K, Abell AN, Midland AA, Zawistowski JS, et al. Dynamic reprogramming of the kinome in response to targeted MEK inhibition in triplenegative breast cancer. Cell 2012 Apr 13;149(2):307-321. 
Appendix 


\title{
Video Article \\ Quantitative Measurement of Invadopodia-mediated Extracellular Matrix Proteolysis in Single and Multicellular Contexts
}

\author{
Karen H. Martin, Karen E. Hayes, Elyse L. Walk, Amanda Gatesman Ammer, Steven M. Markwell, Scott A. Weed \\ Department of Neurobiology and Anatomy, Program in Cancer Cell Biology, Mary Babb Randolph Cancer Center, West Virginia University \\ Correspondence to: Scott A. Weed at scweed@hsc.wvu.edu
}

URL: http://www.jove.com/video/4119/

DOI: $10.3791 / 4119$

Keywords: Cellular Biology, Issue 66, Cancer Biology, Anatomy, Molecular Biology, Biochemistry, invadopodia, extracellular matrix, gelatin, confocal microscopy, quantification, oregon green

Date Published: $8 / 27 / 2012$

Citation: Martin, K.H., Hayes, K.E., Walk, E.L., Ammer, A.G., Markwell, S.M., Weed, S.A. Quantitative Measurement of Invadopodia-mediated Extracellular Matrix Proteolysis in Single and Multicellular Contexts. J. Vis. Exp. (66), e4119 10.3791/4119, DOI : 10.3791/4119 (2012).

\section{Abstract}

Cellular invasion into local tissues is a process important in development and homeostasis. Malregulated invasion and subsequent cel movement is characteristic of multiple pathological processes, including inflammation, cardiovascular disease and tumor cell metastasis ${ }^{1}$ Focalized proteolytic degradation of extracellular matrix (ECM) components in the epithelial or endothelial basement membrane is a critical step in initiating cellular invasion. In tumor cells, extensive in vitro analysis has determined that ECM degradation is accomplished by ventral actin-rich membrane protrusive structures termed invadopodia ${ }^{2,3}$. Invadopodia form in close apposition to the ECM, where they moderate ECM breakdown through the action of matrix metalloproteinases (MMPs). The ability of tumor cells to form invadopodia directly correlates with the ability to invade into local stroma and associated vascular components ${ }^{3}$

Visualization of invadopodia-mediated ECM degradation of cells by fluorescent microscopy using dye-labeled matrix proteins coated onto glass coverslips has emerged as the most prevalent technique for evaluating the degree of matrix proteolysis and cellular invasive potential ${ }^{4.5}$. Here we describe a version of the standard method for generating fluorescently-labeled glass coverslips utilizing a commercially available Oregon Green-488 gelatin conjugate. This method is easily scaled to rapidly produce large numbers of coated coverslips. We show some of the common microscopic artifacts that are often encountered during this procedure and how these can be avoided. Finally, we describe standardized methods using readily available computer software to allow quantification of labeled gelatin matrix degradation mediated by individual cells and by entire cellular populations. The described procedures provide the ability to accurately and reproducibly monitor invadopodia activity, and can also serve as a platform for evaluating the efficacy of modulating protein expression or testing of anti-invasive compounds on extracellular matrix degradation in single and multicellular settings.

\section{Video Link}

The video component of this article can be found at http://www.jove.com/video/4119/

\section{Protocol}

\section{Production of Oregon Green 488-gelatin Coated Coverslips}

1. Prepare an unlabeled $5 \%(\mathrm{w} / \mathrm{w})$ stock gelatin/sucrose solution by adding $1.25 \mathrm{~g}$ gelatin and $1.25 \mathrm{~g}$ sucrose in PBS to a final volume of $50 \mathrm{ml}$. Warm the stock gelatin solution to $37^{\circ} \mathrm{C}$ and ensure it is entirely melted before use. Store the final mixture at $4{ }^{\circ} \mathrm{C}$.

2. Clean $13 \mathrm{~mm}$ diameter \#1 glass coverslips by placing an individual coverslip into each well of a 24 well plastic tissue culture plate. Add $500 \mu$ of $20 \%$ nitric acid to each well and incubate for $30 \mathrm{~min}$. Aspirate the nitric acid solution and wash coverslips three times with deionized water.

3. Coat coverslips with $500 \mu \mathrm{l}$ of $50 \mu \mathrm{g} / \mathrm{ml}$ poly-L-lysine (prepared from $0.1 \%$ stock solution and diluted in deionized water) to each well for 20 min at room temperature. Aspirate the solution and wash three times with PBS. Poly-L-lysine coating facilitates even coating and bonding of the overlying labeled gelatin.

4. Add $500 \mu \mathrm{l}$ of $0.5 \%$ glutaraldehyde (made fresh before use) to each well and incubate the 24 well plates on ice for 15 min. Aspirate and wash three times with cold PBS. Be sure to remove all traces of PBS prior to gelatin coating. Keep plates on ice during all washes until gelatin is added.

5. Reconstitute the Oregon Green 488-conjugated gelatin as per manufacturer's protocol and warm it and the unlabeled $5 \%$ gelatin/sucrose solution from (1.1) to $37^{\circ} \mathrm{C}$. Dilute one part Oregon Green 488 gelatin into eight parts of unlabeled gelatin/sucrose (i.e.; $500 \mu$ l of Oregon Green 488 gelatin into $4 \mathrm{ml}$ of $5 \%$ gelatin mixture). Pipet $100 \mu \mathrm{l}$ of the diluted 488 -gelatin mixture (kept at $37^{\circ} \mathrm{C}$ ) onto each coverslip, using enough gelatin to coat the coverslip without manual spreading (which can lead to uneven coverslip coating as shown in Figure $3 \mathbf{B}$ ). It is important to keep the diluted 488 -gelatin mixture at $37^{\circ} \mathrm{C}$ during the coating procedure to prevent premature solidification. From this step forward the coverslips should be kept in the dark as much as possible to avoid potential photobleaching. Other ECM proteins conjugated to different fluorophores can be substituted for Oregon Green 488 gelatin (see Discussion). 
6. Once all coverslips are coated in a single plate, hold the 24 well plate at an angle and remove excess gelatin from each well by vacuum aspiration. Incubate coated coverslips in the dark for $10 \mathrm{~min}$ at room temperature.

7. Wash the coverslips three times with PBS, then add $500 \mu \mathrm{l}$ of freshly made $5 \mathrm{mg} / \mathrm{ml}$ sodium borohydride $\left(\mathrm{NaBH}_{4}\right)$ for $15 \mathrm{~min}$ at room temperature to reduce and inactivate residual glutaraldehyde. Sodium borohydride is effervescent, and small bubbles will be evident on and around each coverslip.

8. Remove the $\mathrm{NaBH}_{4}$ solution by vacuum aspiration with a quick sweeping motion around the outside of each well. Take care not to pick up any floating coverslips that became detached from the bottom of the tissue culture plate during $\mathrm{NaBH}_{4}$ treatment. Detached coverslips that float to the top may be gently pushed back down to the well bottom, but care must be taken to avoid damaging the protein coating. Wash each well three times with PBS and then incubate coverslips in $70 \%$ ethanol for $30 \mathrm{~min}$ at room temperature.

9. Using sterile technique, transfer the coverslip-containing plates to a type IIA/B cell culture laminar flow hood and rinse coverslips three times with sterile PBS. At this point coverslips can be stored in PBS protected from light at $4{ }^{\circ} \mathrm{C}$ for at least two months.

10. Transfer coverslips to be used for degradation assays to an empty well of a new 24 well plate by careful removal using a sterile needle and forceps. Equilibrate coverslips for 1-24 hr with complete media appropriate to the specific cell type being assayed. Care must be taken not to invert the coverslip or scratch the gelatin coating (see Figure $3 \mathbf{B}$ ).

\section{Plating and Processing of Cells on Oregon Green 488-gelatin Coated Coverslips to Assay ECM Degradation}

1. Seed $3-5 \times 10^{4}$ cells onto a coverslip within each well of the 24 well plate.

2. Conduct a time course study to determine optimal times required for invadopodia degradation activity for the particular cell line/type of interest. Most invasive cells require a time between 4-24 $\mathrm{h}$ for degradation to become apparent, although this range can vary widely and should be empirically determined. To synchronize invadopodia activity, cells can be treated with MMP inhibitors (e.g., GM 6001) for a desired time period, then wash out the inhibitor to allow invadopodia activity to proceed (for example, see ${ }^{6}$ ).

3. Rinse coverslips three times with PBS, then fix cells with $500 \mu \mathrm{l}$ of $10 \%$ buffered formalin phosphate for 15 min. Rinse three times with PBS and permeabilize for 4 min with $0.4 \%$ Triton X-100 in PBS. Rinse three times with PBS to remove the Triton X-100.

4. Label cells using any standard protocol for immunofluorescence staining (see ${ }^{7}$ for example) by co-labeling cells with fluorescent conjugated phalloidin to visualize actin filaments ( $\mathrm{F}$-actin) and for a known marker protein that localizes to invadopodia (e.g; cortactin ${ }^{5}, \mathrm{TKS}^{8}{ }^{2}$ or $\mathrm{N}$ WASp $^{9}$ ).Remember to avoid using 488-labeled secondary antibodies or GFP-labeled proteins if using Oregon Green 488 or FITC-labeled gelatin to prevent signal interference.

5. Mount stained coverslips onto glass microscope slides by carefully inverting the coverslip and placing it on a drop of ProLong Gold antifade or similar reagent.

6. To assess matrix degradation, image cells in appropriate channels using a conventional fluorescent or confocal microscope. Gelatin degradation is visualized as darker areas on the coverslip due to proteolytic removal of the fluorescent gelatin (Figure 4A). Labeling of cells for actin and an invadopodia marker protein allows for confirmation of invadopodia at sites of matrix degradation in merged images (Figure 4A).

7. Degradation activity can also be monitored in real time by live cell imaging with fluorescent-tagged recombinant proteins to track invadopodia formation and matrix degradation ${ }^{5,10,11}$.

\section{Quantification of Fluorescent Gelatin Degradation by Measuring Normalized Matrix Degradation}

This analysis provides the normalized area of matrix degradation relative to the area of the cells or the number of cells. It is useful for analyzing entire microscopic fields of view where multiple cells are present that have been collectively treated with siRNA, growth factors or therapeutic agents. For this analysis, images collected at lower magnification are sufficient to efficiently collect information about populations of cells.

1. Open the images in Image $\mathrm{J}^{12}$. ImageJ for microscopy can be downloaded from http://www.macbiophotonics.ca/imagej/.

2. Check the scale information by choosing the menu command "Analyze/Set Scale." This information will import automatically with many file formats, but can be entered manually if required. Proper scaling is necessary to report measurements in microns rather than pixels.

3. Select the appropriate measurements to track by choosing "Analyze/Set Measurements." Check Area and Limit to Threshold.

4. Calculate the area of degradation using the fluorescent gelatin image (Figure 5A).

5. Threshold the image ("Image/Adjust/Threshold") to set the upper and lower pixel intensity values to select the areas of degradation (highlighted in red; Figure 5B). In subsequent images, use the Set button in the Threshold window to set the same threshold for all images as an objective means to select degradation area.

6. In some cases, the coverslip may not be perfectly flat when images are acquired. This causes the intensity of the gelatin to change across the image. If this variation creates problems when thresholding the image, correct for uneven illumination across the gelatin by subtracting the background ("Process/Subtract Background") or by filtering with a bandpass filter ("Process/FFT/Bandpass Filter") or a pseudo flatfield filter ("Process/Filters/Pseudo Flatfield") until the background intensity is uniform.

7. Measure the area of matrix degradation ("Analyze/Analyze Particles"). In the Analyze Particles window, choose a particle size $>0$ to remove noise from the selection. Show Outlines to identify regions of interest (ROls). Check Display Results and Summarize to show measurements. If the drawing has specifically outlined all of the areas of degradation (Figure 5C), copy the Total Area measurement into a spreadsheet. If other objects were selected (such as debris), record only the areas of the relevant ROIs.

8. Calculate the cell area using the phalloidin stained (F-actin) image (Figure 5D).

9. Threshold the image ("Image/Adjust/Threshold") to set the upper and lower pixel intensity values so that the edges of the cells are selected (highlighted in red; Figure $\mathbf{5 E}$ ). In subsequent images, use the Set button in the Threshold window to set the same threshold for all images as an objective means to select cell area. 
10. 10 Measure the area of the cells ("Analyze/Analyze Particles"). In the Analyze Particles window, choose a particle size $>0$ to remove noise from the selection. Show Outlines to identify regions for analysis (Figure 5F). Check Display Results and Summarize to show area measurements. Do not check Include Holes if there are spaces between cells in a cluster so the non-selected pixels within the cluster will not be included in the cell area calculation. Choose OK.

11. Copy the Area results for relevant ROIs into a spreadsheet.

12. Calculate the area of gelatin degradation per total area of cells ${ }^{13}$.

13. An alternative approach would be to report the area of degradation per number of cells from counting nuclei (Figure 5G). This is necessary if manipulations alter the cell area between different compared treatment groups. Automatic counting works best if nuclei are well separated, uniform in intensity and round. Automatically count nuclei ("Plugins/Particle Analysis/Nucleus Counter"). Choose Smallest and Largest Particle Size, a Threshold Method and a Smoothing Method. Check Subtract Background, Watershed Filter, Add Particles to ROI Manager and Show Summary (Figure $\mathbf{5 H}$ ).

14. If nuclei overlap extensively or have an irregular shape or texture, automatic counting may not produce an accurate count (Figure $\mathbf{5 H}$, arrows on right). In this case, manual counting can be facilitated using the cell counter tool ("Plugins/Particle Analysis/Cell Counter"). This will keep count as cells are marked during a manual count (Figure 5I).

15. Copy the number of cells (nuclei) into a spreadsheet. Calculate the area of gelatin degradation per total number of cells.

\section{Quantification of Fluorescent Gelatin Degradation by Individual Cells in a Mixed Cellular Population}

To evaluate matrix degradation resulting from specific cells in a population apart from other cells within the field (e.g., transfected versus nontransfected cells), the procedure in section 3 can be modified to measure the area of degradation under individual cells. An additional fluorescent channel is needed to mark transfected cells. In this instance, higher magnification images and well-separated cells are easier to quantitate.

1. Check the scale information by choosing the menu command "Analyze/Set Scale." Select the appropriate measurements to track by choosing "Analyze/Set Measurements." Check Area and Limit to Threshold.

2. For individual cells that are not touching, identify each cell using the F-actin image (Figure 6A). Threshold the image (see 3.9) (Figure 6B). It is important to capture the edges of the cells, but there can be holes inside that are not included in the threshold. Use the same intensity values across images to select cell boundaries.

3. To measure the area of the cells, use "Analyze/Analyze Particles." In the Analyze Particles window, choose a Size $>0$ (to eliminate noise), Show Outlines, and check Display Results, Add to Manager and Include Holes (to record the entire area inside the outline). Choose OK and record the Area for each cell from the Results window.

4. Identify which cells are transfected (Figure $\mathbf{6 C}$ ).

5. Identify the areas of degradation using the fluorescent gelatin image (Figure 6D). If needed, filter the gelatin image to even background intensity (see 3.6). Threshold to select the areas of degradation, making note of the threshold settings (Figure 6E). On subsequent images, use these same upper and lower intensity values (using the Set button in the Threshold window) for an objective selection of areas of degradation.

6. Measure the areas of degradation under the cells. On the thresholded fluorescent gelatin image, show an outline of the cells by selecting ROIs in the ROI Manager window and selecting Measure (Figure 6F). Record the results and calculate the normalized area of degradation/ cell or cell area.

\section{Representative Results}

The overall schematic for the procedure is shown in Figure 1. The procedure entails preparation of glass coverslips and coating with fluorescently-conjugated gelatin, plating of cells onto the coated coverslips to allow cells to degrade the gelatin, fixing and labeling of cells for fluorescence microscopic analysis, imaging the fluorescent matrix to assess the matrix integrity, and objectively quantifying the degree of gelatin matrix degradation using computer software. 


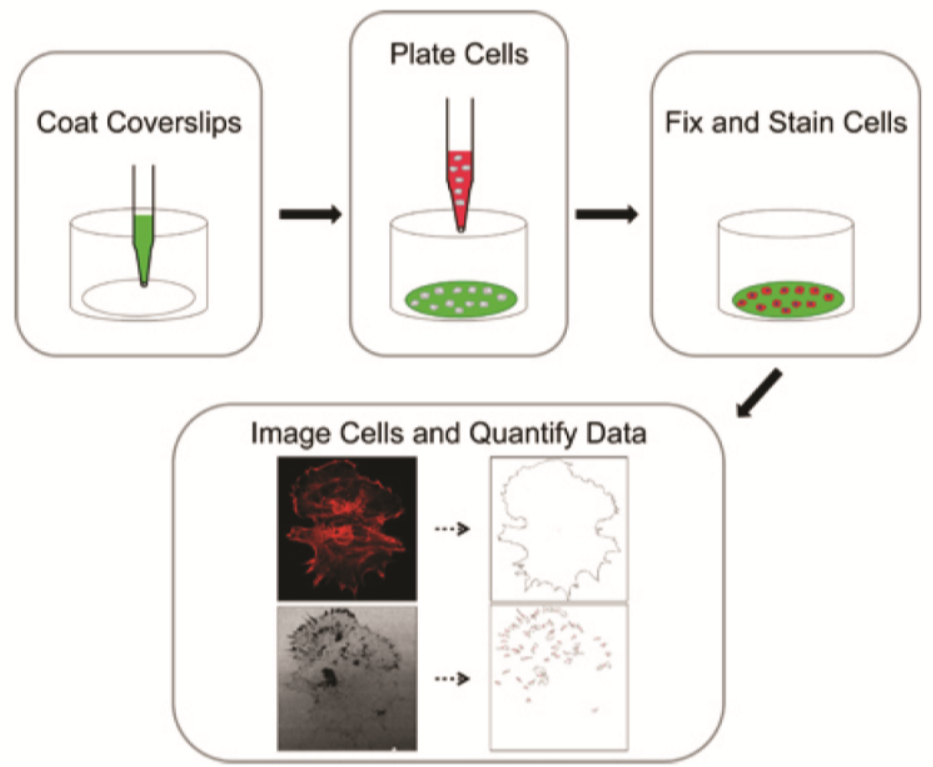

Figure 1. Overall schematic highlighting the key steps involved in fluorescent gelatin coating, cell plating, fixing and immunolabeling, and evaluating matrix proteolysis.

The key procedural steps involved in preparing and coating glass coverslips are outlined in Figure 2.

Prior to gelatin coating
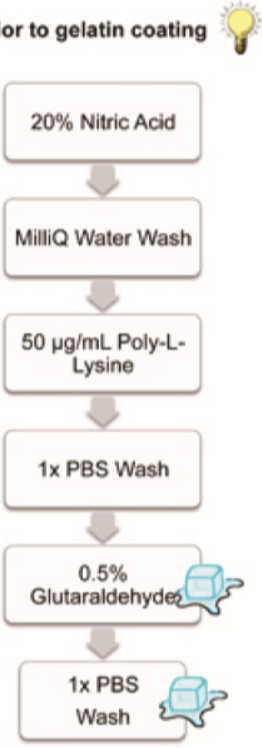

Coating
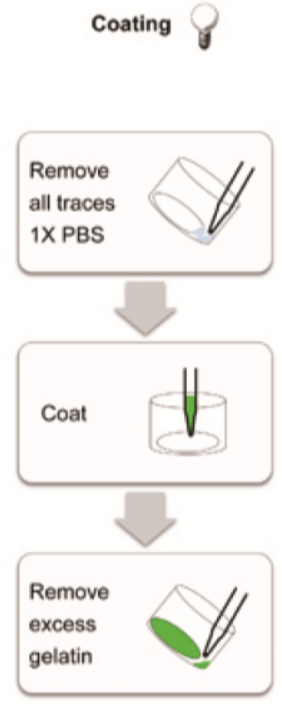

After Coating

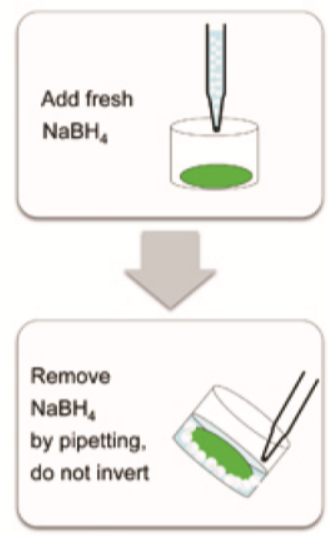

Figure 2. Schematic demonstrating the individual steps involved in preparing glass coverslips for gelatin matrix coating. Steps conducted in the light (lit bulb), on ice (cubes) and in the dark (non-illuminated bulb) are cartoon indicated. Steps conducted in the dark help prevent photobleaching of the fluorescent matrices.

When properly performed, coverslips are evenly coated with Oregon Green 488-conjugated gelatin, displaying homogenous fluorescence when visualized by microscopy (Figure 3A). Typical artifacts that can arise due to improper coating, handling, storage and usage of coated coverslips are shown in Figure 3B. 
A

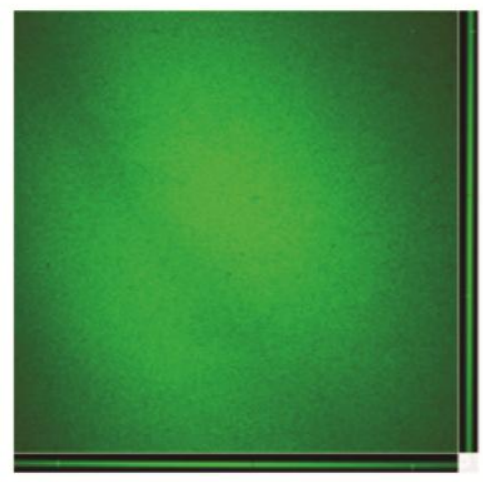

B

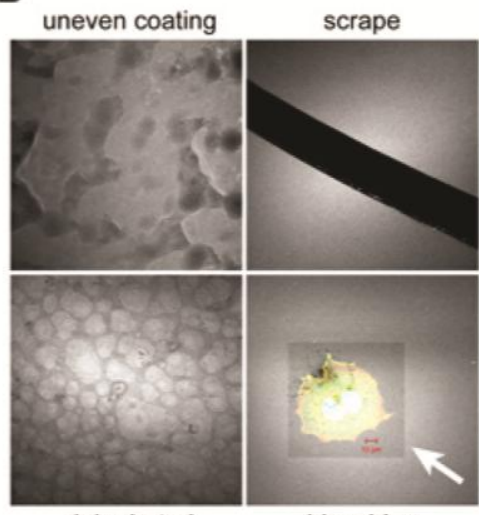

bleaching

Figure 3. Examples of artifacts encountered during gelatin coated coverslip preparation and handling. A. Orthogonal view of a confocal z-stack showing the typical color and consistency of an Oregon Green 488-conjugated gelatin coated coverslip produced using the prescribed protocol. Coverslips should have a homogenous coating $\sim 1-2 \mu \mathrm{m}$ thick as shown in the $\mathrm{X}-\mathrm{Z}$ (bottom) and $\mathrm{Y}$-Z (right) confocal planes. B. Artifacts that can occur during the coating and processing of gelatin-coated coverslips include: Improper covering of the coverslip during the coating process due to poor mixing, manual spreading or partial solidification of the gelatin mixture (uneven coating), removal of the coated matrix by scoring with needles or forceps during handling (scrape), drying of the coverslip surface during prolonged storage periods, resulting in a "cobblestone" appearance (dehydrated) and photobleaching of the fluorescent gelatin surface during imaging due to prolonged or high intensity light exposure (bleaching). White arrow indicates bleached area encompassing a plated OSC19 head and neck squamous carcinoma cell. The Oregon Green 488-conjugated gelatin is pseudocolored white to enhance image contrast. Bar, $10 \mu \mathrm{m}$.

The resulting thin matrices produced during this procedure provide a sensitive means to evaluate the ability of cells to degrade ECM. Figure 4 demonstrates an example of invadopodia activity from an OSC19 cell plated on an Oregon Green-488 conjugated gelatin coverslip and imaged by conventional confocal microscopy as well as by volume-fill image rendering following three dimensional deconvolution. 

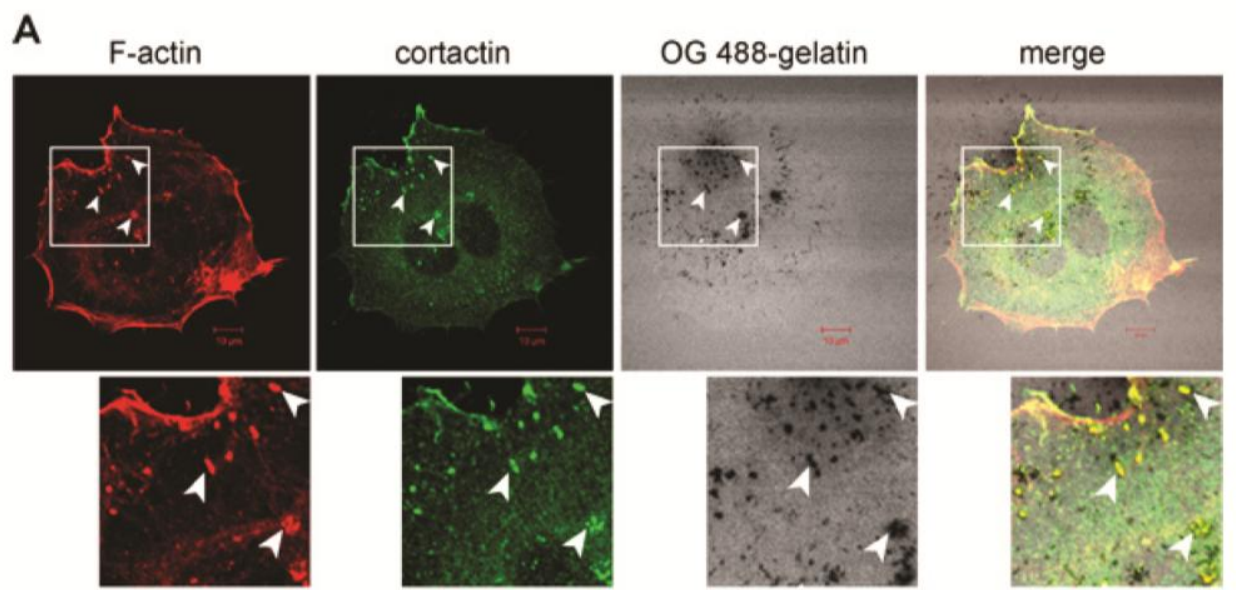

\section{B}

dorsal-edge

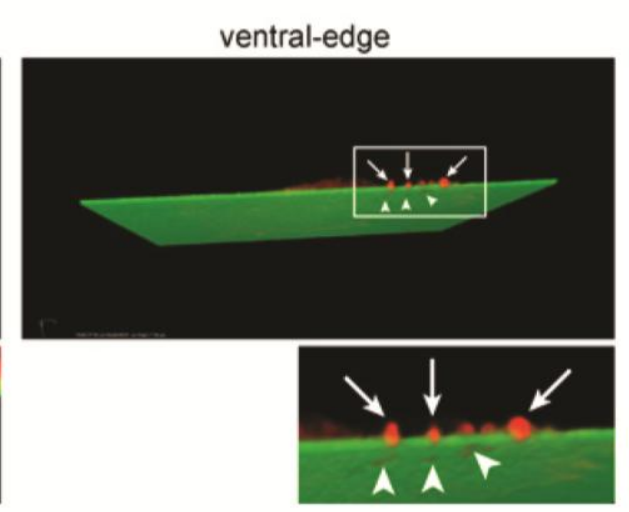

Figure 4. Representative examples of invadopodia matrix degradation activity. A. Visualization of invadopodia and corresponding gelatin matrix proteolysis. OSC19 cells plated on Oregon Green 488-conjugated gelatin coverslips for $10 \mathrm{hr}$ were fixed and labeled with rhodamine-conjugated phalloidin (F-actin) and anti-cortactin antibodies (visualized with an Alexa Fluor 647 secondary antibody and pseudocolored green). Invadopodia are evident as focal cytoplasmic concentrations of $\mathrm{F}$-actin and cortactin that overlap with areas of gelatin clearing (dark holes in the matrix) within the merged image. Boxed regions containing arrowheads indicate individual invadopodia and areas of focal matrix proteolysis as shown in the enlarged regions below. Bar, $10 \mu \mathrm{m}$. B. Volume fill visualization of invadopodia penetration into the ECM. OSC19 cells plated and stained as in (A) were visually rendered by obtaining 23 successive $0.32 \mu \mathrm{m}$ optical $z$-slices totaling $7.04 \mu \mathrm{m}$ for rhodamine-conjugated phalloidin and Oregon Green 488-conjugated gelatin. The native LSM file set for each channel was opened in AutoQuant X2.2 software and a 3D blind deconvolution of each image stack was performed using the recommended settings (10 iterations, medium noise). The processed images were saved as TIFF stacks that were then opened in NIS Elements and rendered as a volume view with alpha blending. The LUTs were adjusted, and a subvolume was created to show an edge inside the cell where invadopodia are present. Dorsal-edge view demonstrates invadopodia (red, arrows) inserted into the underlying gelatin (green). Ventral-edge view shows protrusive invadopodia and areas of gelatin degradation underneath the coverslip as regions of red present in the green matrix (arrowheads). The total image field presented is cropped to $77 \times 65 \mu \mathrm{m}$; the cell is $60 \times 40 \mu \mathrm{m}$.

Figure 5 shows some of the important steps for quantification of normalized gelatin matrix degradation as described in step 3 of the protocol. This procedure is designed to allow for unbiased quantitation of gelatin degradation in an entire field of view, and is suitable for matrix degradation attributed to many cells within the field 

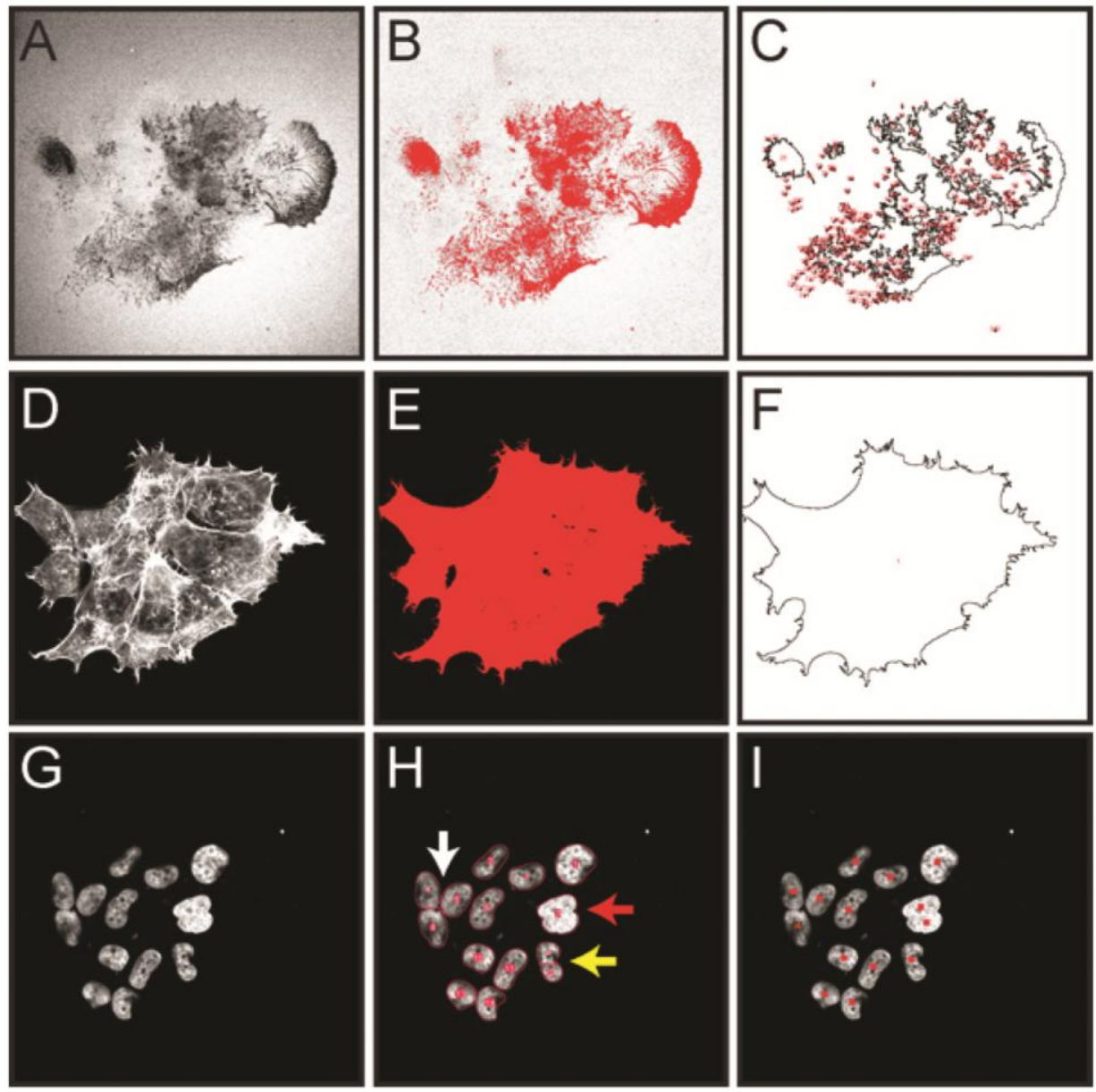

Figure 5. Screen capture images demonstrating key steps in computational-assisted quantification of normalized gelatin degradation for cells within an entire microscopic image as described in protocol step 3. All fluorescent images have been converted to grayscale to better display the red thresholding and ROI markings. A. Image of Oregon Green 488-conjugated gelatin, showing dark areas ("holes") where degradation has occurred (step 3.4). B. Thresholded gelatin image highlighting areas of degradation in red (step 3.5). C. Drawing showing ROls measured for area of degradation (step 3.7). D. Rhodamine phalloidin staining of F-actin (step 3.8). E. Thresholded actin image highlighting total cell area in red (step 3.9). F. Drawing showing cell areas to be measured (step 3.10). G. Image of DAPI-stained cell nuclei (step 3.13). H. Red outlines show results from automatic nuclei counting (step 3.13). The Watershed filter has the potential to separate nuclei that are touching (white arrow). If nuclei overlap extensively, they may not be separated into individual objects (red arrow). If a nucleus has an irregular shape, it may be separated into multiple objects (yellow arrow). I. Results from marking nuclei during a manual count using the cell counter tool (step 3.14).

Figure 6. demonstrates select steps involved in quantifying fluorescent gelatin degradation by individual cells within a mixed cellular population as described in protocol step 4 . Here, matrix degradation by transfected cells can be analyzed within a mixed population of transfected and nontransfected cells. 

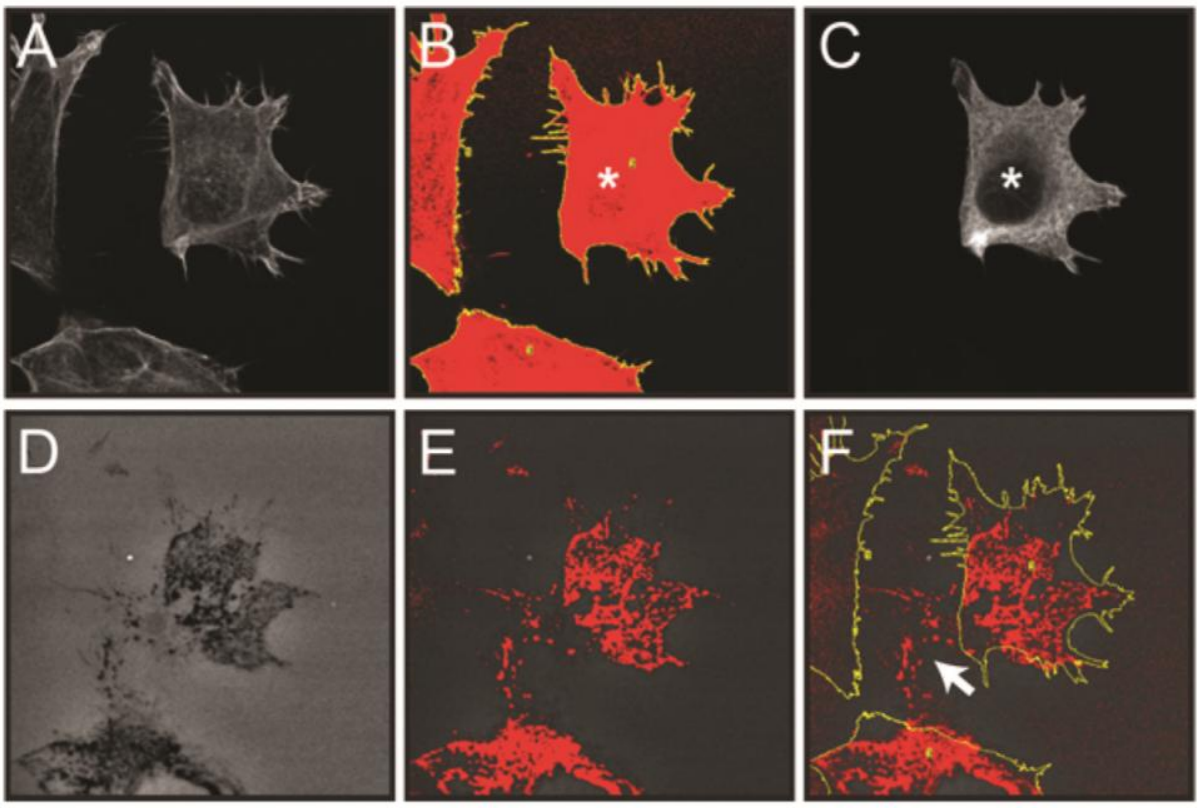

Figure 6. Screen capture images of steps involved in quantifying gelatin degradation from individual transfected cells within a cell population. Quantification of a single transfected OSC19 cell overexpressing recombinant cortactin fused to the FLAG epitope tag is shown as an example. All fluorescent images have been converted to grayscale to better display the red thresholding and yellow ROI markings. A. Confocal image of three cells labeled with rhodamine-phalloidin (step 4.2). B. Drawing of total cell area based on F-actin staining following application of the Threshold and Analyze Particles functions (step 4.2-3). C. Confocal image of anti-FLAG immunolabeling of the cell population demonstrating a single cell expressing FLAG-tagged cortactin (marked with *) (step 4.4). D. Image of Oregon Green 488-conjugated gelatin, showing dark areas ("holes") where degradation has occurred (step 4.5) E. Thresholded gelatin image highlighting dark areas of degradation in red (step 4.5). F. Thresholded gelatin image overlaid with cell outlines from panel B (step 4.6). Note that only the thresholded pixels within the cell outlines are counted in the analysis. Areas of degradation outside the current cell location (white arrow) result from cell migration across the gelatin over time and are not included in the analysis.

\section{Discussion}

The ability to visualize cells degrading the extracellular matrix has aided in discovering the molecular mechanisms employed in the early steps of cell invasion. Pioneered by Wen-Tien Chen in the early 1980 ' $s^{4.14,15}$, coating fluorescently labeled extracellular proteins on glass coverslips for subsequent microscopic analysis has emerged as the primary technique in evaluating invadopodia function across a wide range of cell types. The prescribed protocol demonstrates the basic method used for preparing gelatin-coated coverslips that form a collagenous layer less than $2 \mu \mathrm{m}$ thick suitable for detection of extracellular matrix degradation by cells in most conventional fluorescent and confocal microscopes ${ }^{11,16-18}$, similar to what has been previously described ${ }^{19-21}$. These properties allow for rapid production of coated coverslips capable of detecting the initial onset of matrix degradation. The sensitivity afforded by the resulting thin gelatin matrix on the underlying hard glass surface likely aids in promoting invadopodia formation as a response to the high inherent stiffness of the overall matrix environment ${ }^{22}$. However, these matrices are not well suited for analysis of invadopodia elongation or additional morphological evaluation that has been achieved using thicker $(30-100 \mu \mathrm{m})$ gelatin layers with similar methodology, coated transwells or electron microscopy $\mathrm{y}^{20,23,24}$

We have found that pre-conjugated commercially produced Oregon Green 488 gelatin allows for rapid experimental set up and consistent, reproducible results. However, alkaline borate conjugation of fluorescein isothiocyanate (FITC) to unlabeled gelatin remains a popular and inexpensive method for producing fluorescent gelatin conjugates ${ }^{20}$. Fibronectin is also used as an alternative matrix protein for labeling and coverslip coating ${ }^{4.9}$, and in some cases investigators have used labeled fibronectin layered onto unlabeled gelatin coated coverslips to create denser matrices ${ }^{11.25}$. Other matrices could be used, depending on the specifics of the cell type. In addition to dyes in the green $488 \mathrm{~nm}$ spectrum, a wide range of fluorophores have also been used with manual coupling methods to generate coverslips with different fluorescence spectra, including rhodamine ${ }^{21,26}$, Alexa Fluor $350^{24}, 546^{21}, 568^{5,11}, 594^{27}$ and $647^{5}$ dyes. Such conjugates are easily adaptable for use in the prescribed protocol, providing the flexibility for utilizing specific ECM protein-dye combinations suitable for most any imaging filter set.

The techniques described herein provide the necessary detailed steps for utilizing Image J to quantify gelatin matrix degradation attributed to individual cells in a heterogeneous population or to entire cell groups as published previously. ${ }^{6.28}$. Proprietary software has also been successfully employed for the same purpose $e^{5,25}$. In this protocol, the area of matrix degradation is normalized to either the total area of the cells or the total number of cells (nuclei) in the field. Generally, both options for normalization will give the same result (ELW, data not shown). However, if different cell lines having different sized cells are being compared or if the experimental treatment causes cells to change size, then it may be 
more accurate to normalize to cell number. On the other hand, many cancer cell lines have a high percentage of multi-nucleated cells, in which case total cell area may be a more accurate parameter for normalization. Also, if only part of a cell is captured in an image (Figure 6), it may be better to normalize to cell area rather than underestimate the degradation potential for an individual cell. It is important to optimize the image analysis to best suit the characteristics and nuances of the specific experimental setup.

For determining cell numbers in a crowded field, counting nuclei is often the method of choice. ImageJ has a nucleus counter plugin for automatic counting. One option in this tool is the Watershed filter. This filter will help separate nuclei that are touching by segregating them into individual objects (Figure $\mathbf{5 H}$, white arrow). However, this filter may not be able to separate nuclei that overlap extensively (Figure $\mathbf{5 H}$, red arrow). In addition, if a nucleus has an irregular shape and large variations in intensity, the filter may separate a single nucleus into multiple objects (Figure $\mathbf{5 H}$, yellow arrow). Therefore, it is important to try different thresholding and smoothing methods in this plugin to determine the best parameters for analysis. If the automatic counting does not produce accurate numbers, the cell counter plugin can facilitate manual counting of cells or nuclei.

In cases utilizing transient transfection, images will often contain a mixture of cells expressing or not expressing a protein of interest (Figure 6). In this scenario, it is not always apparent which cells were responsible for creating areas of matrix degradation. This is especially true if the cells are migrating across the gelatin. To be consistent in the analysis, it is important to only measure the degraded areas directly underneath each cell. By thresholding to select the dark areas in the matrix and using the actin to generate cell outlines, only the degraded areas under the cells will be quantitated. This procedure will exclude degraded areas outside of cell boundaries from analysis (Figure $6 \mathrm{~F}$, arrow). The assay may require optimization to select a time point that allows sufficient time for degradation before the cells have had a chance to move.

Numerous methods have been developed to quantitate invadopodia formation and function. In addition to matrix degradation, other frequently reported parameters include determining the number of invadopodia per cell, the percentage of cells displaying invadopodia within a given population, and the number of "immature" or "pre" non-degrading invadopodia compared to "mature" invadopodia capable of degrading the $\mathrm{ECM}^{11,13,25,26}$. The method(s) of choice for invadopodia evaluation depend on inherent characteristics of each cell type. For instance, counting the number of invadopodia per cell or determining the percentage of cells containing invadopodia is a straightforward approach that works well if the analyzed cells contain just a few prominent invadopodia, but becomes more difficult in cells that have dozens of invadopodia or where invadopodia may be small and difficult to detect. Using the degradation assay makes it possible to calculate the percentage of pre-invadopodia vs. mature invadopodia in single cells or in a population by comparing the total number of cells with invadopodia to the percentage that are degrading matrix. If there is a discrepancy where fewer cells are degrading matrix compared to cells displaying invadopodia, it may indicate that these cells are forming pre-invadopodia that were incapable of matrix degradation at the time the cells were fixed.

Whatever method combination is chosen for analysis, it is important to quantify the desired invadopodia characteristics as objectively as possible. When collecting images on the microscope, choose fields by looking at cells (actin), rather than the fluorescent matrix, to avoid bias from preferentially selecting areas with high levels of degradation. Multiple images should be acquired to ensure a fair representation of the cell population. Images should also be acquired at an appropriate magnification. For uniform populations of cells, lower magnification can be used to collect more cells as long as the areas of degradation can still be resolved. Higher magnification images are preferred to measure areas under individual cells and to resolve individual invadopodia. When areas are being quantitated, thresholding images based on intensity is more objective than manually choosing the area of the matrix to measure. In all cases, a sufficient number of cells from multiple independent experiments should be analyzed to give statistically meaningful, reproducible results.

Disclosures

No conflicts of interest declared.

\section{Acknowledgements}

This work was supported by endowment funds from the West Virginia University Mary Babb Randolph Cancer Center. We thank Susette Mueller (Georgetown University) and Laura Kelley for early advice and assistance. The use of the West Virginia University Microscopy Imaging Facility (supported by the Mary Babb Randolph Cancer Center and, NIH grants P20 RR16440, P30 RR032138 and P30 GM103488) is gratefully acknowledged.

\section{References}

1. Ridley, A.J. Life at the leading edge. Cell. 145, 1012-1022, [pii] S0092-8674(11)00652-0 doi:10.1016/j.cell.2011.06.010 (2011).

2. Murphy, D.A. \& Courtneidge, S.A. The 'ins' and 'outs' of podosomes and invadopodia: characteristics, formation and function. Nat. Rev. Mol. Cell Biol. 12, 413-426, [pii] nrm3141 doi:10.1038/nrm3141 (2011).

3. Linder, S., Wiesner, C., \& Himmel, M. Degrading Devices: Invadosomes in Proteolytic Cell Invasion. Annu. Rev. Cell. Dev. Biol., doi:10.1146/ annurev-cellbio-092910-154216 (2010).

4. Chen, W.T., Chen, J.M., Parsons, S.J., \& Parsons, J.T. Local degradation of fibronectin at sites of expression of the transforming gene product pp60src. Nature. 316, 156-158 (1985).

5. Artym, V.V., Zhang, Y., Seillier-Moiseiwitsch, F., Yamada, K.M., \& Mueller, S.C. Dynamic interactions of cortactin and membrane type 1 matrix metalloproteinase at invadopodia: defining the stages of invadopodia formation and function. Cancer Res. 66, 3034-3043, [pii] 66/6/3034 doi:10.1158/0008-5472.CAN-05-2177 (2006).

6. Ayala, I., et al. Multiple regulatory inputs converge on cortactin to control invadopodia biogenesis and extracellular matrix degradation. J. Cell Sci., (2008)

7. Ammer, A.G., et al. Saracatinib impairs head and neck squamous cell carcinoma invasion by disrupting invadopodia function. J. Cancer Sci. Ther. 1, 52-61, doi:10.4172/1948-5956.1000009 (2009). 
8. Seals, D.F. et al. The adaptor protein Tks5/Fish is required for podosome formation and function, and for the protease-driven invasion of cancer cells. Cancer Cell. 7, 155-165 (2005).

9. Yamaguchi, H., et al. Molecular mechanisms of invadopodium formation: the role of the N-WASP-Arp2/3 complex pathway and cofilin. J. Cell Biol. 168, 441-452 (2005)

10. Kopp, P., et al. The kinesin KIF1C and microtubule plus ends regulate podosome dynamics in macrophages. Mol. Biol. Cell. 17, 2811-2823, [pii] E05-11-1010 doi:10.1091/mbc.E05-11-1010 (2006).

11. Oser, M., et al. Cortactin regulates cofilin and N-WASp activities to control the stages of invadopodium assembly and maturation. J. Cell. Biol. 186, 571-587 (2009).

12. Rasband, W.S. ImageJ. U.S. National Institutes of Health., (1997-2011).

13. Kelley, L.C., et al. Oncogenic Src requires a wild-type counterpart to regulate invadopodia maturation. J. Cell Sci. 123, 3923-3932, [pii] jes. 075200 doi: $10.1242 / j \mathrm{js} .075200$ (2010).

14. Chen, W.T. \& Singer, S.J. Fibronectin is not present in the focal adhesions formed between normal cultured fibroblasts and their substrata. Proc. Natt. Acad. Sci. U.S.A. 77, 7318-7322 (1980)

15. Chen, W.T., Olden, K., Bernard, B.A., \& Chu, F.F. Expression of transformation-associated protease(s) that degrade fibronectin at cell contact sites. J. Cell Biol. 98, 1546-1555 (1984).

16. Bharti, S., et al. Src-dependent phosphorylation of ASAP1 regulates podosomes. Mol. Cell Biol. 27, 8271-8283 (2007).

17. Albrechtsen, R., Stautz, D., Sanjay, A., Kveiborg, M., \& Wewer, U. M. Extracellular engagement of ADAM12 induces clusters of invadopodia with localized ectodomain shedding activity. Exp. Cell Res. 317, 195-209, [pii] S0014-4827(10)00457-X doi:10.1016/j.yexcr.2010.10.003 (2011).

18. Scott, R.W., et al. LIM kinases are required for invasive path generation by tumor and tumor-associated stromal cells. J. Cell Biol. 191, 169-185, [pii] jcb.201002041 doi:10.1083/jcb.201002041 (2010).

19. Mueller, S.C., Yeh, Y., \& Chen, W.T. Tyrosine phosphorylation of membrane proteins mediates cellular invasion by transformed cells. J. Cell. Biol. 119, 1309-1325 (1992).

20. Bowden, E.T., Coopman, P.J., \& Mueller, S.C. Invadopodia: unique methods for measurement of extracellular matrix degradation in vitro. Methods Cell Biol. 63, 613-627 (2001).

21. Baldassarre, M., et al. Dynamin participates in focal extracellular matrix degradation by invasive cells. Mol. Biol. Cell. 14, 1074-1084 (2003)

22. Alexander, N.R., et al. Extracellular matrix rigidity promotes invadopodia activity. Curr. Biol. 18, 1295-1299, [pii] S0960-9822(08)01035-X doi:10.1016/j.cub.2008.07.090 (2008).

23. Artym, V.V., Yamada, K.M., \& Mueller, S.C. ECM degradation assays for analyzing local cell invasion. Methods Mol. Biol. 522, 211-219, doi:10.1007/978-1-59745-413-1_15 (2009).

24. Schoumacher, M., Goldman, R.D.., Louvard, D., \& Vignjevic, D.M. Actin, microtubules, and vimentin intermediate filaments cooperate for elongation of invadopodia. J. Cell Biol. 189, 541-556, [pii] jcb.200909113 doi:10.1083/jcb.200909113 (2010).

25. Clark, E.S., Whigham, A.S., Yarbrough, W.G., \& Weaver, A.M. Cortactin is an essential regulator of matrix metalloproteinase secretion and extracellular matrix degradation in invadopodia. Cancer Res. 67, 4227-4235, [pii] 67/9/4227 doi:10.1158/0008-5472. CAN-06-3928 (2007).

26. Yamaguchi, H., et al. Phosphoinositide 3-kinase signaling pathway mediated by p110alpha regulates invadopodia formation. J. Cell Biol. 193, 1275-1288, [pii] jcb.201009126 doi:10.1083/jcb.201009126 (2011).

27. Li, A., et al. The actin-bundling protein fascin stabilizes actin in invadopodia and potentiates protrusive invasion. Curr. Biol. 20, 339-345, [pii] S0960-9822(09)02159-9 doi:10.1016/j.cub.2009.12.035 (2010)

28. Yamaguchi, H., et al. Phosphatidylinositol 4,5-bisphosphate and PIP5-kinase lalpha are required for invadopodia formation in human breast cancer cells. Cancer Sci. [pii] CAS1574 doi:10.1111/j.1349-7006.2010.01574.x (2010). 


\section{Materials List for:}

\section{Quantitative Measurement of Invadopodia-mediated Extracellular Matrix Proteolysis in Single and Multicellular Contexts}

Karen H. Martin, Karen E. Hayes, Elyse L. Walk, Amanda Gatesman Ammer, Steven M. Markwell, Scott A. Weed

Department of Neurobiology and Anatomy, Program in Cancer Cell Biology, Mary Babb Randolph Cancer Center, West Virginia University

Correspondence to: Scott A. Weed at scweed@hsc.wvu.edu

URL: http://www.jove.com/video/4119/

\section{Materials}

\begin{tabular}{|l|l|l|l|}
\hline Name & Company & Catalog Number & Comments \\
\hline gelatin & Sigma & G1890 & Porcine skin \\
\hline sucrose & Fisher & BP220 & \\
\hline 12 mm coverslips & Fisher & $50-121-5159$ & \\
\hline 24 well plates & Fisher & $08-772-1$ & BD Falcon \\
\hline nitric acid & Ricca & R5326000 & $20 \%$ solution \\
\hline poly-L-lysine & Electron Microscopy Sciences & $19320-B$ & $0.1 \%$ solution \\
\hline glutaraldehyde & Sigma & G7526 & $8 \%$ solution \\
\hline $\begin{array}{l}\text { Oregon Green 488-conjugated } \\
\text { gelatin }\end{array}$ & Invitrogen & G-13186 & \\
\hline sodium borohydride & Sigma & 213462 & \\
\hline Triton X-100 & Fisher & BP151 & \\
\hline rhodamine-phalloidin & Invitrogen & R415 & \\
\hline anti-cortactin (clone 4F11) & Millipore & $05-180$ & \\
\hline Anti-FLAG antibody & Millipore & MAB3118 & \\
\hline $\begin{array}{l}\text { Alexa Fluor 647 Goat anti-mouse } \\
\text { IgG }\end{array}$ & Invitrogen & A21235 & \\
\hline ProLong Gold antifade & Invitrogen & P36930 & \\
\hline LSM 510 Confocal Microscope & Zeiss & & http://www.macbiophotonics.ca/ \\
\hline imageJ software & Public domain & & \\
\hline AutoQuant X2.2 software & Media Cybernetics & & \\
\hline NIS Elements software & Nikon & & \\
\hline
\end{tabular}




\title{
Video Article \\ Multi-photon Imaging of Tumor Cell Invasion in an Orthotopic Mouse Model of Oral Squamous Cell Carcinoma
}

\author{
Amanda Gatesman Ammer ${ }^{1}$, Karen E. Hayes ${ }^{1}$, Karen H. Martin ${ }^{1}$, Lingqing Zhang ${ }^{2}$, George A. Spirou ${ }^{3}$, Scott A. Weed ${ }^{1}$ \\ ${ }^{1}$ Department of Neurobiology and Anatomy, Program in Cancer Cell Biology, Mary Babb Randolph Cancer Center, West Virginia University \\ ${ }^{2}$ Sensory Neuroscience Research Center, West Virginia University \\ ${ }^{3}$ Departments of Otolaryngology and Physiology, Center for Neuroscience, West Virginia University \\ URL: http://www.jove.com/video/2941/ \\ DOI: $10.3791 / 2941$ \\ Keywords: Medicine, Issue 53, Invasion, mouse model, two-photon microscopy, tongue, orthotopic, head and neck cancer
}

Date Published: $7 / 25 / 2011$

This is an open-access article distributed under the terms of the Creative Commons Attribution-NonCommercial License, which permits noncommercial use, distribution, and reproduction, provided the original work is properly cited.

Citation: Gatesman Ammer, A., Hayes, K.E., Martin, K.H., Zhang, L., Spirou, G.A., Weed, S.A. Multi-photon Imaging of Tumor Cell Invasion in an Orthotopic Mouse Model of Oral Squamous Cell Carcinoma. J. Vis. Exp. (53), e2941 10.3791/2941, DOI : 10.3791/2941 (2011).

\section{Abstract}

Loco-regional invasion of head and neck cancer is linked to metastatic risk and presents a difficult challenge in designing and implementing patient management strategies. Orthotopic mouse models of oral cancer have been developed to facilitate the study of factors that impact invasion and serve as model system for evaluating anti-tumor therapeutics. In these systems, visualization of disseminated tumor cells within oral cavity tissues has typically been conducted by either conventional histology or with in vivo bioluminescent methods. A primary drawback of these techniques is the inherent inability to accurately visualize and quantify early tumor cell invasion arising from the primary site in three dimensions. Here we describe a protocol that combines an established model for squamous cell carcinoma of the tongue (SCOT) with two-photon imaging to allow multi-vectorial visualization of lingual tumor spread. The OSC-19 head and neck tumor cell line was stably engineered to express the F-actin binding peptide LifeAct fused to the mCherry fluorescent protein (LifeAct-mCherry). Fox $1^{\text {nu/nu }}$ mice injected with these cells reliably form tumors that allow the tongue to be visualized by ex-vivo application of two-photon microscopy. This technique allows for the orthotopic visualization of the tumor mass and locally invading cells in excised tongues without disruption of the regional tumor microenvironment. In addition, this system allows for the quantification of tumor cell invasion by calculating distances that invaded cells move from the primary tumor site. Overall this procedure provides an enhanced model system for analyzing factors that contribute to SCOT invasion and therapeutic treatments tailored to prevent local invasion and distant metastatic spread. This method also has the potential to be ultimately combined with other imaging modalities in an in vivo setting.

\section{Video Link}

The video component of this article can be found at http://www.jove.com/video/2941/

Protocol

\section{Cell Lines, Vector Construction and Lentiviral Production}

1. Human head and neck tumor cells lines (OSC19 or UMSCC1) were cultured in complete media consisting of DMEM (Cellgro cat \# 50-003PB) supplemented with 10\% fetal bovine serum FBS (Hyclone cat \# SH30070.03), 1\% penicillin/streptomycin (Cellgro cat \#30-002-Cl), and $1 \%$ non-essential amino acids (Cellgro cat \# 25-025-Cl)

2. To transfer the LifeAct-mCherry coding sequence into the pLL7.0 lentiviral vector, the Sbf1 recognition site in the parent mCherry cDNA was altered by introducing three silent mutations into the recognition sequence using site-directed mutagenesis (Stratagene cat \# 200518-5). The resulting modified LifeAct-mCherry sequence was then PCR amplified with flanking EcoR1/Sbf1 sites and subcloned into pLL7.0 to generate the pLL7.0-LifeAct-mCherry construct.

\section{2. pLL7.0-LifeAct-mCherry Virus Production}

1. Viral production was conducted according to the Lentiviral Expression Systems manual (System Bioscience version 2-051018).

2. The packaging cell line 293 T/17 cells (ATCC cat \# CRL-11268) was grown to $40 \%$ confluence in the same complete media used for HNSCC lines.

3. Cells were transfected with the pLL7.0-LifeAct-mCherry, psPAX2, and pVSV-G vectors in a 3:2:1 ratio, respectively using CalPhos ( Clontech cat \# 631312).

4. After 24 hours, the initial media from the transfection was replaced with fresh media.

5. Media was then collected and replenished every 12 hours for 72 hours and stored at $4^{\circ} \mathrm{C}$ 


\section{Production of Head and Neck Cell Lines with Stable LifeAct-mCherry Expression}

1. The collected media was spun at $2000 \mathrm{rpm}$ for 10 minutes at $4^{\circ} \mathrm{C}$.

2. One $\mathrm{ml}$ of clarified media containing virus was directly added to OSC19 or UMSCC1 cells for 12 hours. Cells were then rinsed, and an additional one $\mathrm{mL}$ of virus was added for another 12 hour period.

3. Cells were treated with media containing $200 \mathrm{mg} / \mathrm{ml}$ puromycin for two weeks to select resistant colonies.

4. Surviving clones were screened visually for LifeAct-mCherry expression by fluorescence microscopy. Individual positive colonies were typsinized using sterile $3 \mathrm{~mm}$ cloning discs (Fisher cat \# 0790710A).

5. Positive cells were maintained in media containing $200 \mathrm{mg} / \mathrm{ml}$ puromycin until frozen back or used for orthotopic injection.

\section{Orthotopic Tumor Xenograft Formation}

1. All animal procedures were conducted in accordance with a protocol (09-0821) approved by the West Virginia University Animal Care and Use Committee.

2. LifeAct-mCherry expressing tumor cells were trypsinized, centrifuged and $2.5 \times 10^{4}$ cells were resuspended in $50 \mu \mathrm{L}$ complete media.

3. Tumor cells were loaded into a one $\mathrm{ml}$ syringe attached to a $271 / 2$ gauge needle.

4. Female athymic Fox $1^{\mathrm{nu} / \mathrm{hu}}$ mice 8 weeks of age (Harlan Laboratories) were anesthetized with combination of $80 \mathrm{mg} / \mathrm{kg} \mathrm{ketamine}$ and $10 \mathrm{mg} / \mathrm{kg}$ xylazine. Anesthetized mice were maintained between $37-40^{\circ} \mathrm{C}$ on a heating pad.

5. Using sterile forceps, the tip of the tongue was gently grasped and carefully pulled out of the oral cavity.

6. Cells were slowly injected into one side of each tongue to create a bulbous mass in the tongue center, avoiding the lingual arteries.

7. Mice were injected with $2.1 \mathrm{mg} / \mathrm{kg}$ yohimbine and returned to the heating pad where they were monitored for $2-3 \mathrm{hours}$ during recovery from anesthesia.

8. Once revived, mice were placed into sterile cages containing a soft transgenic dough diet (Bioserve cat \# S3472).

9. Mice were weighed every 2-3 days and monitored visually for tumor onset.

\section{Preparation of Mouse Tongues for Ex-vivo Imaging}

1. Mice harboring tumors at different time points (typically 2-4 weeks post-injection) were euthanized by carbon dioxide inhalation.

2. Tongues were extracted, rinsed with 1 X PBS and attached to one side of a conventional paraffin tissue embedding cassette (StatLab Cat \# $\mathrm{H} 104$, , using monofilament sewing thread from a local hobby shop and a size 8 sewing needle.

3. Once the tongues were immobilized, the entire cassette assembly was placed in a $30 \mathrm{~mm}$ tissue culture dish and immersed in $1 \mathrm{X}$ PBS.

4. Processed tongues were immediately used for two-photon excitation microscopy.

\section{Imaging of Tongue Tumors with Two-photon Microscopy}

1. Tongue cassettes were submerged in a $60 \mathrm{~mm}$ dish containing $1 \mathrm{X}$ PBS secured in a custom designed holder on a retractable cantilever arm (Chamber Shuttle, Siskiyou instruments) positioned under the objective of an upright microscope (Moveable Microscope (MOM); Sutter Instruments)

2. A 40X/0.8NA water dipping objective lens was placed directly on or over visible tumor lesions. Tongues were imaged by two-photon microscopy with the Ti:sapphire laser (Mira, Coherent) intensity at $60 \mathrm{~mW}$ and input wavelength of $755 \mathrm{~nm}$ to optimize the mCherry signal.

3. Serial $1 \mathrm{~mm}$ laser scanning images were collected at $1 \mu \mathrm{m}$ incremental depths over a total tissue depth between 15 and $100 \mu \mathrm{m}$ (depending on tumor volume). Images were captured using Scanlmage, an open source program based on the MATLAB platform that was developed by the Karel Svoboda laboratory (Janelia Farms, HHMI). Scanlmage generates a two-channel output of raster scan patterns to control the $\mathrm{x} / \mathrm{y}$ galvanometric scan mirrors, and at the same time captures a maximum four-channel signal input simultaneously from photomultiplier tubes (PMTs) through a data acquisition board (PCl-6610S, National Instruments). The PMT signals are amplified by low noise current preamplifiers (SR570, Stanford Research System) before feeding into the NI-DAQ board for display on the monitor screen. Scanlmage collects z-stack images by controlling the $\mathrm{z}$-axis of the objective and collects time-lapse images in a single or cycle mode. Images were saved in a single TIFF file with 16 bit depth.

\section{Image Analysis using Amira Software}

1. Amira imaging for tumor quantification: In Aimra software, open the TIFF file containing the set of z-stack images.

2. Highlight the file name, select and apply the Voltex function to generate a three dimensional rendering. A large primary tumor image with several smaller, dissociated invasive groups (IG) of collectively invaded cells is typically apparent in the rendered image (see Figure $6 \mathrm{~A}$ ).

3. To select the primary tumor mass, select "Open Data", then "Labeling", then "Label Field".

4. Thresholding the primary tumor- select all z-stack images and scroll through the images in the z-plane to ensure inclusion of only the primary tumor mass within three dimensions. This is typically the largest sized image in the field and often appears segmented as the imaged is traversed through the $z$-plane.

5. Use the magic wand/threshold function to correct background fluorescence and eliminate it from the image without discarding any of the tumor signal. Highlight the "Inside File" label and click the \# button. Select the "All Slices" check box. This produces a colored border around the thresholded primary tumor area in every z-stack increment.

6. To select IGs, choose the "New" function. Select a single IG and ensure it is not associated with the primary tumor by scrolling through the zplane.

7. Select "All Slices" and click the \# button. Rename the file (ex: IG 1)

8. Repeat the thresholding procedure in 7.4 and the highlighting step in 7.5 for the determined IG. Select an outline color different than the color used to denote the primary tumor mass. 
9. Repeat as necessary for each additional IG throughout the image, scanning through the Z-plane to ensure all IGs are denoted. Using different colors for each identified IG aids in future identification on the image.

10. Once the primary tumor and all IGs are selected, select "Segmentation" from the pulldown menu, then select "Material Statistics". This provides the volume measurement as well as the $X, Y$ and $X$ tumor core coordinates for the primary tumor in order to calculate distances of IGs from the central tumor point.

11. With the volumes calculated, determine the distance for each IG from the central core of the primary tumor. Select "Segmentation", then "Material Statistics". This step calculates all measurements into pixels. Convert pixels to micrometers based on the calibration of the microscope objective and any additional increases in magnification (i.e.; zoom functions). For the microscope and settings used in these experiments, the $40 X$ objective was used with no zoom, giving a calibration of 1 pixel $=0.298 \mu \mathrm{m}$. Import data into an Excel spreadsheet, which gives parameters for the primary tumor in three dimensions (X1, Y1, Z1) and for each IG (ex: X2, Y2, Z2 for the first IG).

12. The invaded distance in microns for each IG from the center of the primary tumor is calculated using the formula $\sqrt{ }\left((X 2-X 1)^{2}+(Y 2-Y 1)^{2}+(Z 2-\right.$ $\mathrm{Z} 1)^{2}$ ).

13. The tumor invasive index $\left(T_{1}\right)$ is calculated using the formula $T_{1}=N_{T} \times V_{T} \times D_{T}$, where $N_{T}=$ the total number of IGs in the image, $V_{T}=$ the total volume of all IGs, $D_{T}=$ total distance traveled of all invasive groups from the center of the primary tumor.

\section{Three dimensional renderings with Nikon NIS-Elements Software}

1. Three dimensional renderings with greater topographical detail can be generated by importing the original 16-bit monochrome TIFF files of the entire tumor image into the Nikon NIS-Elements software package (Nikon, Melville, NY). Select "File", then "ND", then "Create ND File from File Sequence". Select the TIFF z-series image stack and specify the appropriate step size.

2. Calibrate the ND document in the xy plane. Specify the size of one pixel using a manual calibration.

3. Choose the "Volume View". Use the "HQ" function to calculate additional slices in the z-plane for higher quality. In the "3D Renderer Settings", use the "Advanced Renderer" with a quality of "Ultra High Details" and "Full Resolution". Choose "Alpha Blending" to accentuate the tumor surfaces.

4. Optimize the three-dimensional image for presentation. Zoom in on the tumor and associated IGs and crop as needed. Rotate the image in the xyz planes and adjust the LUTs.

5. Capture the image for presentation. Select "Edit- Create View Snapshot ( 8 bit RGB)". Name and save file accordingly

\section{Representative Results}

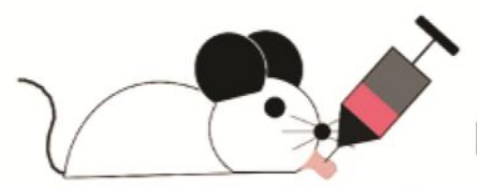

LifeAct-mCherry labeled HNSCC cells
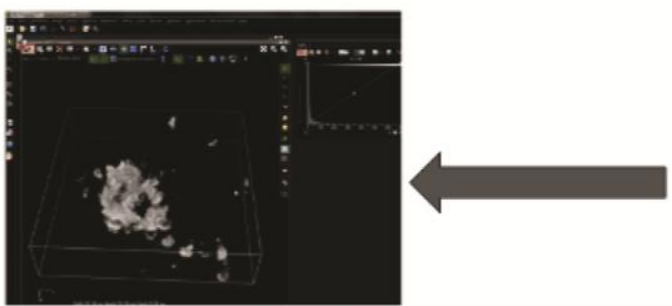

Image Reconstruction and Analysis

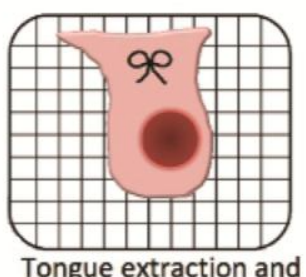

Tongue extraction and preparation for two-photon

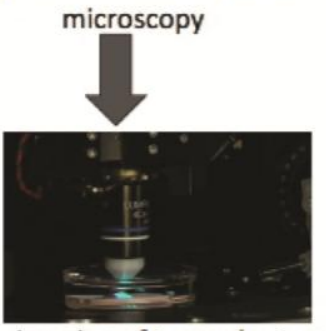

Imaging of tumor by two photon microscopy

Figure 1. Overall schematic illustrating key steps in orthotopic tongue tumor production and in situ two photon imaging. 


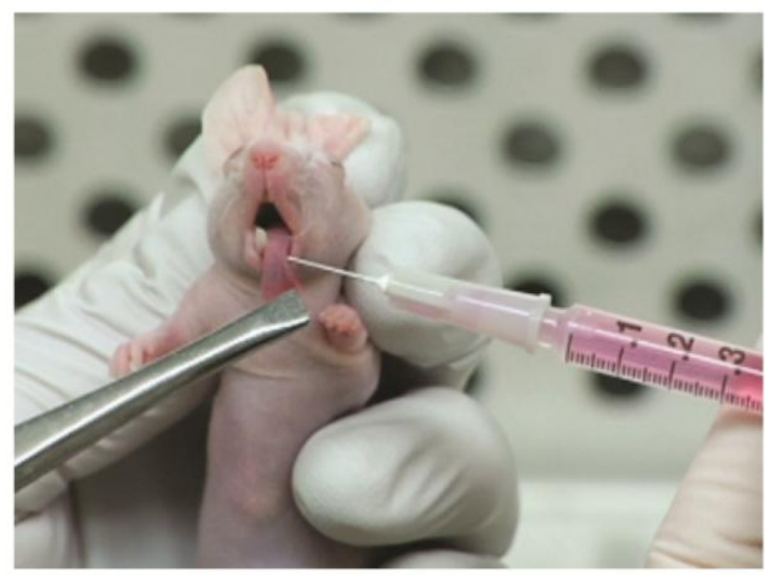

Figure 2. Injection of LifeAct-mCherry expressing OSC19 cells into the mouse tongue.

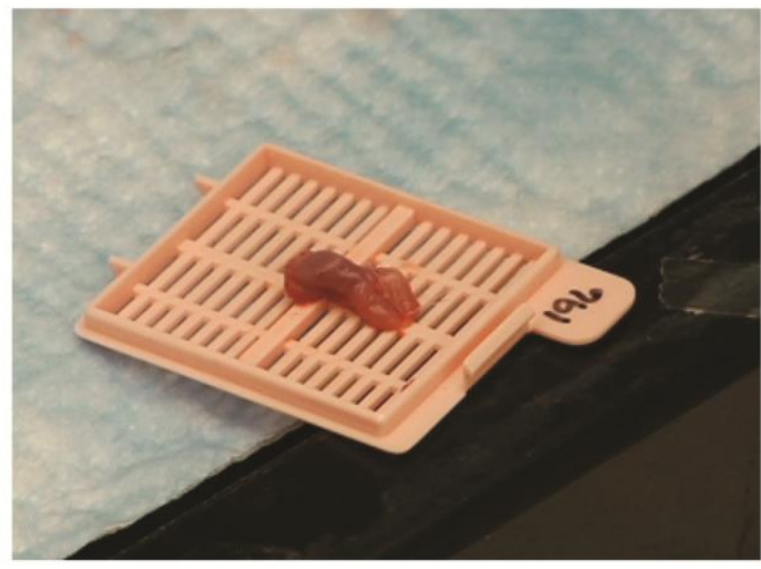

Figure 3 Resected tumor-containing mouse tongue prepared for two photon imaging.

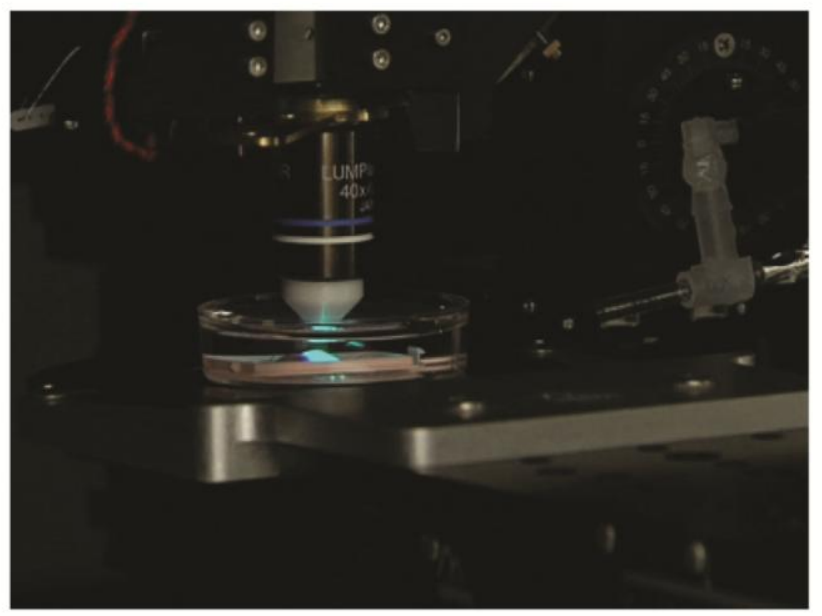

Figure 4. Orientation of a tumor-containing tongue in position on a two photon microscopy ready for imaging. 


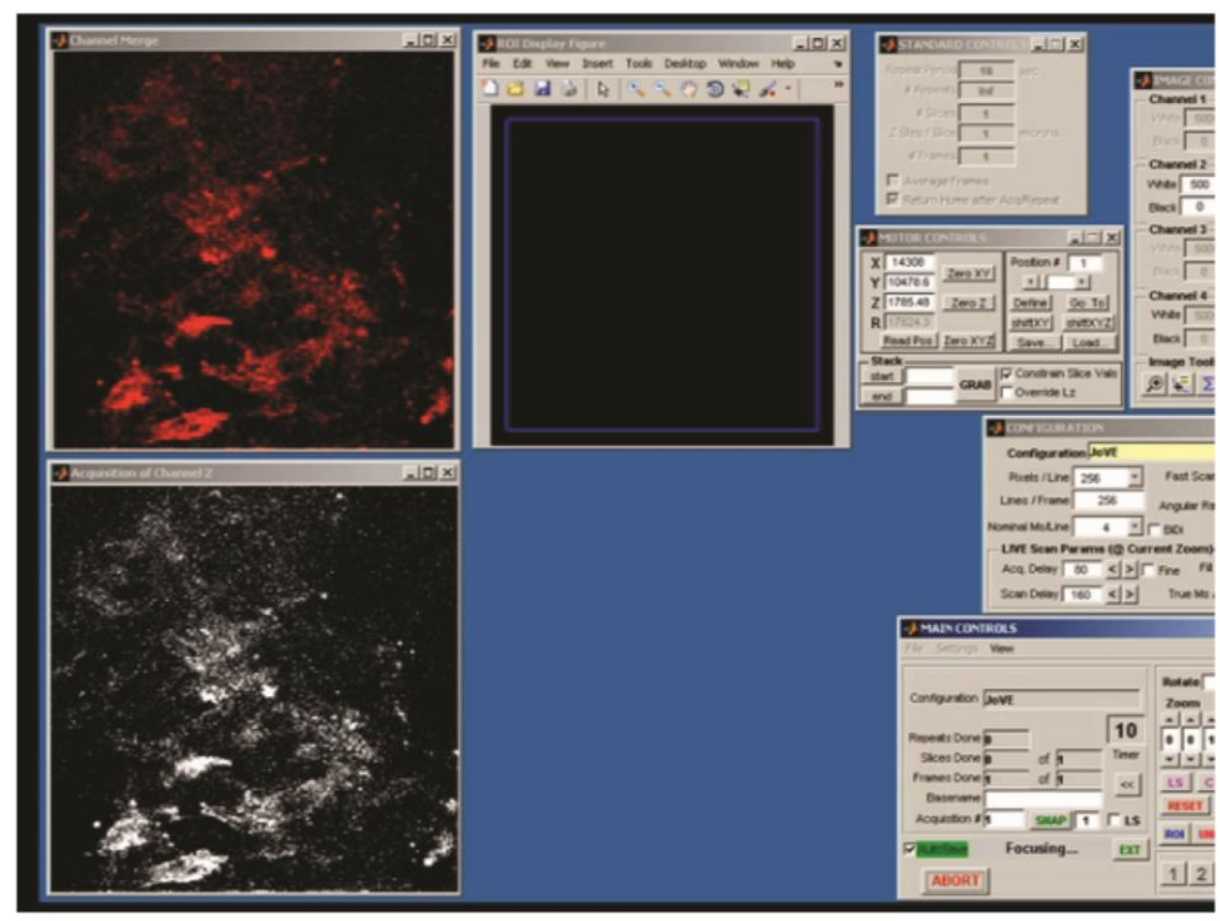

Figure 5. Representative screen shot from Scanlmage demonstrating raw data acquisition of the initial two-photon image 
A

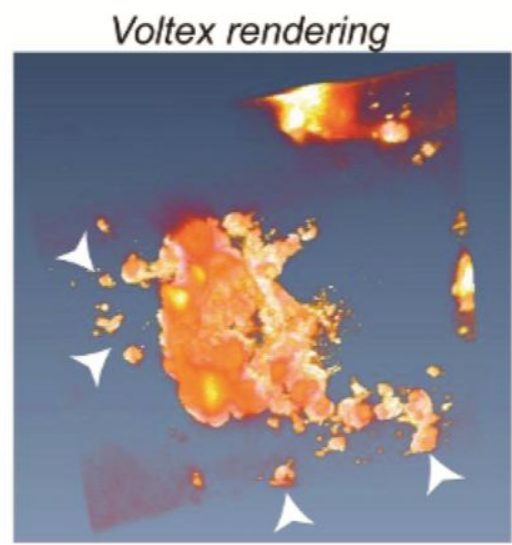

C

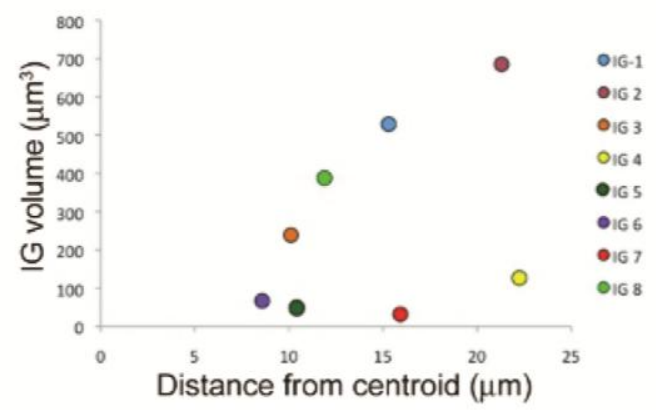

B

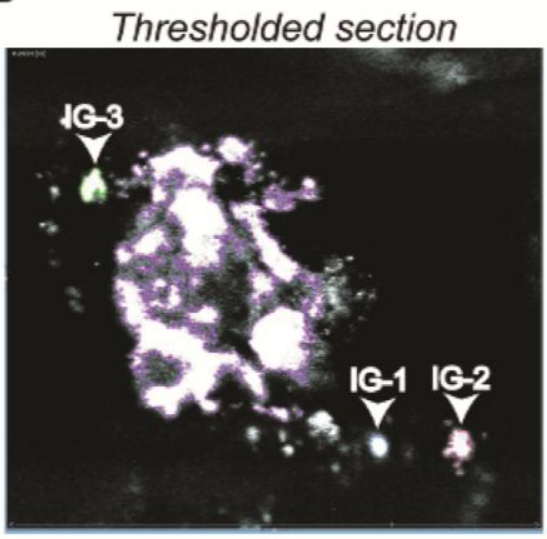

Figure 6. Image analysis and quantification of tumor invasion in an orthotopic OSC19 tumor. Representative screen shot images of Amira Voltex rendering $(A)$ and a single thresholded $z$-section $(B)$ with the primary primary tumor outlined in purple, invasive group 1 (IG-1) outlined in blue, IG-2 outlined in red and IG-3 outlined in green as an example of the identification procedure. Arrowheads denote IGs in (A) and (B). C. Plots of volume versus invaded distance for eight individual IGs used to calculate the tumor invasion index (Ti).

A

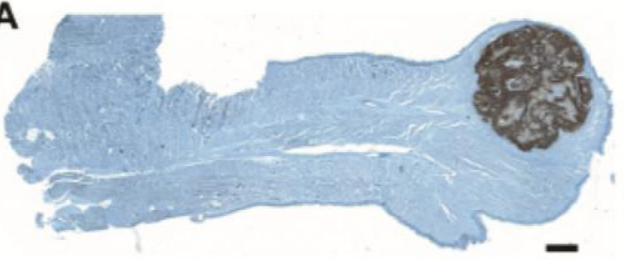

B

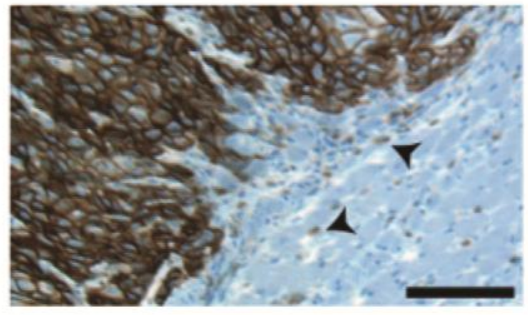

C

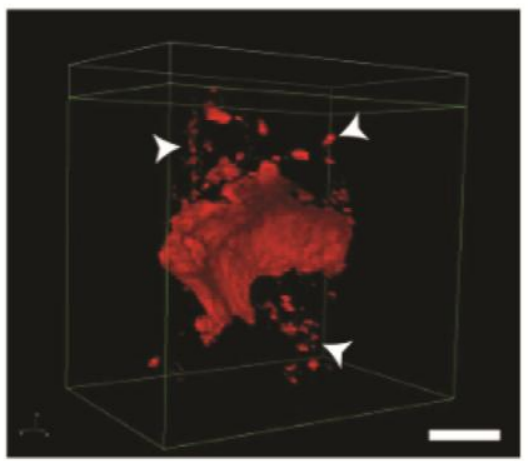

Figure 7. Representative images of tumors and invaded tumor groups from this protocol compared with images from a conventional IHC approach. A. Paraffin section of an entire mouse tongue harboring a UMSCC1 orthotopic tumor. Immunohistochemical staining was conducted using conventional procedures with a primary antibody against the HNSCC-specific cell marker emmprin (Zymed; cat \# 34-5600) at 1:1000 dilution and visualized by using the OmniMap DAB anti-Rb detection kit (Ventana cat \# 760-149) followed by iron hematoxylin staining. Images encompassing the total tongue area were collected individually at $4 X$ magnification on an Olympus ZX70 Provis microscope with an Optronics MicroFire CCD camera and reconstructed using the StereolNvestigator imaging package (MBF Bioscience). The tumor is evident at the tongue 
tip. An enlarged region showing the invasive front and individual tumor cell groups (arrowheads) is shown in (B). C. Nikon NIS-Elements rendering of a representative OSC19 tumor. Enhanced contour detail and clear visualization of IGs (arrowheads) is evident. Bars in all images = $100 \mu \mathrm{m}$.

\section{Discussion}

Orthotopic mouse models have proven useful for studying many aspects of head and neck cancer ${ }^{1,2}$. We have combined a well established orthotopic system of $\mathrm{SCOT}^{3}$ with two-photon microscopy imaging of mCherry-labeled cells as a system to study the early events of head and neck tumor cell invasion. In this procedure, we have noted that cells can leak from the site of tumor injection, especially in mice six weeks or younger due to insufficient tongue size. We use older mice to avoid this issue. The larger tongue size with older mice also aids in avoiding rupture of a lingual artery and excessive hemorrhaging from the tongue. Tumor take is greatly enhanced when the tongue dramatically swells at the site of initial injection. This swelling subsides within one-two hours as the injected fluid is absorbed. Tumor growth appears evident one-two weeks post injection with the indicated injected cell number as a small white bump on the tongue surface. We also note that at the $40 \mathrm{X}$ objective used in our studies presented here, we cannot distinguish individual tumor cells but do identify IGs as invasive cell clusters that recapitulate the typical mode of HNSCC invasion.

To date this model has been extensively used for testing the role of specific molecules as well as several anti-tumor drugs on SCOT growth an invasion, with efficacy measured by cervical lymph node metastasis monitored using IHC or bioluminescent methods ${ }^{4-7}$. Tumors formed in this system manifest close to the tongue surface (Figure 7), allowing the application of two-photon microscopy to image entire tumors in situ as invasive multi-cellular clusters. The procedure can also be utilized to visualize tumor invasion with cellular resolution. Two-photon microscopy has been previously utilized to study experimental treatments for head and neck cancer in orthotopic ${ }^{8,9}$ and xenograft ${ }^{8,10}$ models. However, there are two major differences between these reports and our protocol. First, these studies use extracellular labels to target/identify head and neck tumor cells, possibly limiting detection only to tumor cells with ample access to the circulation. Second, invasion of tumor cells close to the primary site that likely recapitulates early metastatic activity was not assessed as an experimental parameter. Our protocol provides the ability to directly quantify tumor cell invasion at any point during tumor progression in mouse tongues. While the method described here describes the procedure using dissected tongues, we are currently in the process of adapting this method to image tumor invasion in live mice for use in combination with bioluminescence to simultaneously monitor early invasion, local lymph node involvement and distant metastasis in the same animal. Alterations to the protocol for in vivo imaging require designing of an appropriate stage for positioning and maintaining mice during imaging, as well as a practical system to properly irrigate the oral cavity of anesthetized mice during the imaging procedure. Once optimized, these adaptations will provide the ability to study the role of potential pro-invasive molecules and the testing anti-invasive compounds on local invasion and more distant metastatic involvement in animals over extended time periods.

Disclosures

The authors have nothing to disclose.

\section{Acknowledgements}

Supported by a subproject of NIH grant P20 RR16440 and a bridge grant from the West Virginia University Office of Research and Graduate Education to S. Weed. Technical assistance from L. Lopez-Skinner during the early phases of project development is gratefully acknowledged The authors are also grateful for technical assistance and OSC19 cells from J. Myers and M. Younes (Department of Head and Neck Surgery, M.D. Anderson Cancer Center, Houston, TX); P. Turner and K. Secrest (West Virginia University Department of Pathology Tissue Bank) for histological processing and procedures, R. Wysolmerski (West Virginia University, Department of Neurobiology and Anatomy) for the LifeActmCherry construct and J. Bear (University of North Carolina) for the pLL7.0 lentiviral vector. The use of the West Virginia University Microscopy Imaging Facility (supported by NIH grant P20 RR16440 and the Mary Babb Randolph Cancer Center) and its non-linear optical microscopy laboratory (NLOM; supported by a joint collaboration between the West Virginia University Center for Neuroscience and the West Virginia University Department of Physics/West Virginia Nanoscience Initiative) is also gratefully acknowledged. The NLOM is supported in part by NIH grant P30 RR031155 to the Center for Neuroscience.

\section{References}

1. Sano, D., \& Myers, J. N. Xenograft models of head and neck cancers. Head Neck Oncol. 1, 32, doi:1758-3284-1-32 [pii] 10.1186/1758-3284-1-32 (2009).

2. Kim, S. Animal models of cancer in the head and neck region. Clin Exp Otorhinolaryngol. 2, 55-60, doi:10.3342/ceo.2009.2.2.55 (2009)

3. Myers, J. N., Holsinger, F. C., Jasser, S. A., Bekele, B. N., \& Fidler, I. J. An orthotopic nude mouse model of oral tongue squamous cell carcinoma. Clin Cancer Res. 8, 293-298 (2002).

4. Sano, D., \& Myers, J. N. Metastasis of squamous cell carcinoma of the oral tongue. Cancer Metastasis Rev. 26, 645-662 (2007).

5. Sano, D. et al. The effect of combination anti-endothelial growth factor receptor and anti-vascular endothelial growth factor receptor 2 targeted therapy on lymph node metastasis: a study in an orthotopic nude mouse model of squamous cell carcinoma of the oral tongue. Arch Otolaryngol Head Neck Surg. 135, 411-420, doi:135/4/411 [pii]10.1001/archoto.2009.14 (2009).

6. Kupferman, M. E. et al. TrkB induces EMT and has a key role in invasion of head and neck squamous cell carcinoma. Oncogene. 29, 2047-2059, doi:onc2009486 [pii]10.1038/onc.2009.486 (2010).

7. Ammer, A. G. et al. Saracatinib Impairs Head and Neck Squamous Cell Carcinoma Invasion by Disrupting Invadopodia Function. J Cancer Sci Ther. 1, 52-61, doi:10.4172/1948-5956.1000009 (2009). 
8. Rosenthal, E. L., Kulbersh, B. D., King, T., Chaudhuri, T. R., \& Zinn, K. R. Use of fluorescent labeled anti-epidermal growth factor receptor antibody to image head and neck squamous cell carcinoma xenografts. Mol Cancer Ther. 6, 1230-1238, doi:6/4/1230 [pii]10.1158/1535-7163.MCT-06-0741 (2007).

9. Gleysteen, J. P. et al. Fluorescent labeled anti-EGFR antibody for identification of regional and distant metastasis in a preclinical xenograft model. Head Neck. 30, 782-789, doi:10.1002/hed.20782 (2008).

10. Bhirde, A. A. et al. Targeted killing of cancer cells in vivo and in vitro with EGF-directed carbon nanotube-based drug delivery. ACS Nano 3 , 307-316, doi:10.1021/nn800551s (2009). 


\section{Revisiting the ERK/Src cortactin switch}

Laura C. Kelley, Karen E. Hayes, Amanda Gatesman Ammer, Karen H. Martin and Scott A. Weed*

Department of Neurobiology and Anatomy; Program in Cancer Cell Biology; Mary Babb Randolph Cancer Center; West Virginia University,

Morgantown, WV USA

Key words: cortactin, ERK1/2, Src, N-WASp, phosphorylation, motility, invasion

Submitted: $12 / 06 / 10$

Accepted: $12 / 07 / 10$

DOI: $10.4161 /$ cib.4.2.14420

*Correspondence to: Scott A. Weed; Email: sweed@hsc.wvu.edu

Addendum To: Kelley LC, Hayes KE, Ammer AG, Martin KH, Weed SA. Cortactin phosphorylated by ERK1/2 localizes to sites of dynamic actin regulation and is required for carcinoma lamellipodia persistence. PLoS ONE 2010; 5:13847; PMID: 21079800; DOI: 10.1371/journal.pone.
$\mathrm{T}$ The filamentous $(\mathrm{F})$-actin regulatory protein cortactin plays an important role in tumor cell movement and invasion by promoting and stabilizing actin related protein (Arp) 2/3-mediated actin networks necessary for plasma membrane protrusion. Cortactin is a substrate for ERK1/2 and Src family kinases, with previous in vitro findings demonstrating ERK1/2 phosphorylation of cortactin as a positive and Src phosphorylation as a negative regulatory event in promoting Arp2/3 activation through neuronal Wiskott Aldrich Syndrome protein (N-WASp). Evidence for this regulatory cortactin "switch" in cells has been hampered due to the lack of phosphorylation-specific antibodies that recognize ERK1/2-phosphorylated cortactin. Our findings with phosphorylation-specific antibodies against these ERK1/2 sites (pS405 and pS418) indicate that cortactin can be co-phosphorylated at $405 / 418$ and tyrosine residues targeted by Src family tyrosine kinases. These results indicate that the ERK/Src cortactin switch is not the sole mechanism by which ERK1/2 and tyrosine phosphorylation events regulate cortactin function in cell systems.

Motility-based processes in normal and transformed cells are governed by signal transduction pathways that regulate actin cytoskeletal dynamics. Actin regulatory proteins that serve as substrates downstream of multiple kinase cascades are important intersection points in integrating and controlling motile and invasive activities. Cortactin is an $\mathrm{F}$-actin binding adaptor protein initially identified as a Src substrate in v-Src transformed cells. ${ }^{1,2}$
Subsequent work identified three tyrosine residues within the cortactin proline-rich carboxyl-terminal domain (Y421, Y470 and Y486 in humans) that are phosphorylated by Src and other tyrosine kinases. ${ }^{3,4}$ Analysis of these tyrosine residues using phenylalanine point mutants indicates the importance of their phosphorylation in cell motility and tumor cell metastasis., Tyrosine phosphorylated cortactin localizes within lamellipodia of motile cells and invadopodia in invasive carcinoma cells,${ }^{6,7}$ supporting a functional role in cell movement and invasion.

Cortactin is a substrate for multiple serine/threonine kinases in addition to serving as a tyrosine kinase substrate. ${ }^{8}$ ERK $1 / 2$ is a prominent serine/threonine kinase that phosphorylates cortactin at serine 405 and 418 in response to epidermal growth factor receptor (EGFR) activa-

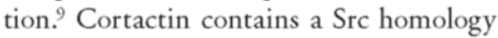
(SH) 3 domain at its carboxyl terminal end that is capable of binding and activating N-WASp, resulting in enhanced Arp2/3 actin nucleation activity.10,11 In 2004, Martinez-Quiles et al. examined the functional impact of ERK $1 / 2$ and Src phosphorylation on the ability of cortactin to regulate N-WASp activity. This report utilized purified protein components in in vitro actin polymerization assays to demonstrate that ERK1/2 phosphorylation substantially enhances the ability of cortactin to bind and activate N-WASp, promoting Arp2/3 actin nucleation. In contrast, Src phosphorylation of cortactin prevents cortactin binding to N-WASp and ablates the ability of phosphomimetic 405/418 cortactin mutants to promote N-WASp activation. These results led to the proposal of an on-off switch mechanism for 


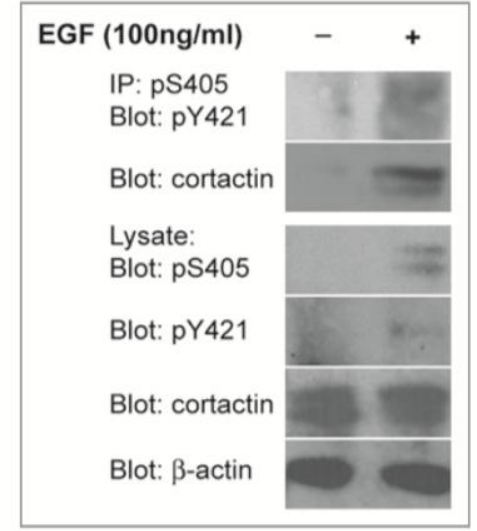

Figure 1. Cortactin is co-phosphorylated on $\mathrm{S} 405$ and Y421. OSC19 oral squamous carcinoma cells serum starved for $24 \mathrm{~h}$ were stimulated with $100 \mathrm{ng} / \mathrm{ml}$ EGF for $20 \mathrm{~min}$ as indicated. Cell lysates were immunoprecipitated with anti-cortactin pS405 antibodies and analyzed by western blotting with anticortactin pY421 and pan-cortactin antibodies. Total cell lysates were evaluated for the presence of pS405 cortactin, pY421 cortactin, total cortactin and $\beta$-actin.

cortactin regulation of N-WASp activity, whereby ERK1/2 cortactin phosphorylation liberates the cortactin $\mathrm{SH} 3$ domain from an undefined autoinhibitory state (presumed to be a $\mathrm{SH} 3$ domain binding site within the proline-rich domain), allowing it to bind and activate N-WASp. Src phosphorylation terminates this activity, enabling cortactin to return to its inactive confirmation. Based on this model, ERK1/2 phosphorylation and Src tyrosine phosphorylation are functionally interdependent events.

Subsequent studies utilizing exogenously expressed phosphomimetic and phosphorylation incompetent S405/418 mutants supports a role for ERK $1 / 2$ cortactin phosphorylation in promoting intracellular actin polymerization ${ }^{12}$ and actin-dependent invadopodia matrix degradation activity. ${ }^{13}$ Our development of site-specific antibodies against cortactin pS405 and pS418 allows for the first direct cellular evaluation of these phosphorylation sites. We demonstrate that pS418 cortactin localizes to lamellipodia and invadopodia in carcinoma cells, supporting the identified role for cortactin in actin polymerization derived from studies with point mutant constructs. Significantly, these antibodies, along with commercially available antibodies against cortactin pY421 (and likely anti-pY470/pY486 or equivalent antibodies), provide a means for directly assessing cortactin serine and tyrosine phosphorylation status derived from cellular preparations. Cortactin is phosphorylated on S405/418 and Y421 in lysates from EGF-stimulated UMSCC2 head and neck squamous carcinoma cells, indicating that ERK1/2 and Src (or other cortactin-targeting tyrosine kinases) are activated and phosphorylate cortactin following EGFR activation. Analysis of phosphorylation-null point mutants with anti-pY421 and pS405 antibodies indicates that ERK1/2 and cortactin tyrosine phosphorylation are not interdependent events, since the inability of cortactin to become tyrosine phosphorylated does not prevent ERK1/2 phosphorylation (and vice versa). Furthermore, cortactin immunoprecipitated with anti-pS405 antibodies is phosphorylated on pY421 (Fig. 1), demonstrating co-phosphorylation of these sites on the same cortactin molecule. These data indicate that at least a subpopulation of cortactin within tumor cells is simultaneously phosphorylated by ERK $1 / 2$ and tyrosine kinases.

Our findings provide evidence that the ERK/Src cortactin switch is not the primary phospho-regulatory cortactin mechanism employed by cells. Recent studies indicate that cortactin tyrosine phosphorylation promotes actin polymerization through recruitment of the adaptor NCK1, which in turn binds and activates N-WASp and Arp2/3 to stimulate actin network formation in invadopodia. ${ }^{7,14,15}$ The concurrent ability of cortactin to activate N-WASp by both tyrosine and ERK1 phosphorylation events allows for amplification of Arp2/3-mediated actin polymerization based on specific signaling input, providing a means for fine-tuning actin regulation at dynamic membrane structures during migration and invasion. Cortactin regulation of actin networks is likely more complex, given the ability of the cortactin $\mathrm{SH} 3$ domain to interact and activate several proteins in addition to N-WASp that signal to control Arp2/3 activation or actin dynamics. $^{16-20}$ The presence of tyrosine ${ }^{21}$ and

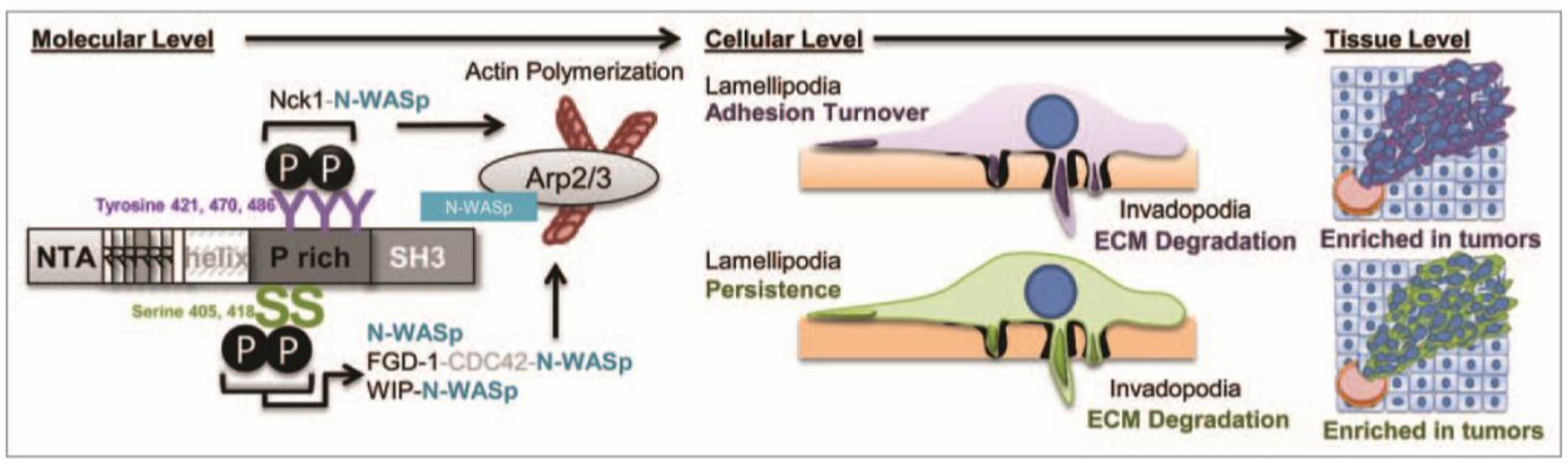

Figure 2. Mechanistic and cellular roles of cortactin tyrosine and serine phosphorylation in tumor cell motility. Tyrosine (purple) and serine (green) phosphorylation events are shown depicting direct and indirect pathways that ultimately result in N-WASp activation. The effects of cortactin serine and tyrosine phosphorylation result in common net outcomes in invadopodia function (ECM degradation), but differ in impact on lamellipodia dynamics as shown. Cortactin phosphorylated on serine and tyrosine residues is enriched in human tumors, where it likely enhances invasive and metastatic capacity. 
serine phosphorylated cortactin in head and neck squamous cell carcinoma tumors suggests that both tyrosine- and serinebased signaling is relevant in neoplastic progression.

In addition to the apparent redundant roles of tyrosine and serine phosphorylation in N-WASp activation, these cortactin phosphorylation events can also have divergent cellular functions in migration. In two-dimensional systems, cortactin tyrosine phosphorylation alters focal adhesion turnover, whereas serine 405/418 phosphorylation stimulates actin polymerization and motility. ${ }^{12}$ Our work extends these findings by demonstrating that serine $405 / 418$ phosphorylation is required for dominant lamellipodia persistence, whereas tyrosine phosphorylation has no effect on lamellipodia dynamics (Ammer and Weed, unpublished data). This suggests that there are context-specific roles for cortactin tyrosine and serine phosphorylation in tumor cell motility (Fig. 2) and is in agreement with the ability of these phosphorylation events to regulate different aspects of endocytic membrane trafficking. ${ }^{22-24}$ Continued deciphering of the complex pathways that impinge on and emanate from serine- and tyrosine-phosphorylated cortactin continues to present interesting and challenging avenues for understanding how these signals are utilized and integrated during different phases of cancer cell motility.

\section{Acknowledgements}

This work was supported by a subproject of NIH grant P20-RR16440 to S.A.W.

\section{References}

1. Wu H, Reynolds AB, Kanner SB, Vines RR, Parsons JT. Identification and characterization of a novel cytoskeleton-associated pp60src substrate. Mol Cell Biol 1991; 11:5113-24.

2. Wu H, Parsons JT. Cortactin, an $80 / 85$-kilodalton pp60src substrate, is a filamentous actin-binding protein enriched in the cell cortex. J Cell Biol 1993; 120:1417-26.

3. Huang C, Liu J, Haudenschild CC, Zhan X. The role of tyrosine phosphorylation of cortactin in the locomotion of endothelial cells. J Biol Chem 1998; 273:25770-6.

4. Ammer AG, Weed SA. Cortactin branches out: roles in regulating protrusive actin dynamics. Cell Motil Cytoskel 2008; 65:687-707.

5. Li Y, Tondravi M, Liu J, Smith E, Haudenschild CC, Kaczmarek M, et al. Cortactin potentiates bone metastasis of breast cancer cells. Cancer Res 2001; 61:6906-11.

6. Head JA, Jiang D, Li M, Zorn LJ, Schaefer EM, Parsons JT, et al. Cortactin tyrosine phosphorylation requires Racl activity and association with the cortical actin cytoskeleton. Mol Biol Cell 2003; 14:3216-29.

7. Oser M, Mader CC, Gil-Henn H, Magalhaes M, Bravo-Cordero JJ, Koleske AJ, et al. Specific tyrosine phosphorylation sites on cortactin regulate Nckldependent actin polymerization in invadopodia. J Cell Sci 2010; 123:3662-73

8. Martin KH, Jeffery ED, Grigera PR, Shabanowitz J, Hunt DF, Parsons JT. Cortactin phosphorylation sites mapped by mass spectrometry. J Cell Sci 2006; 119:2851-3.

9. Campbell DH, Sutherland RL, Daly RJ. Signaling pathways and structural domains required for phosphorylation of EMS1/cortactin. Cancer Res 1999; 59:5376-85.

10. Mizutani K, Miki H, He H, Maruta H, Takenawa T. Essential role of neural Wiskott-Aldrich syndrome protein in podosome formation and degradation of extracellular matrix in src-transformed fibroblasts. Cancer Res 2002; 62:669-74.

11. Martinez-Quiles N, Ho HY, Kirschner MW, Ramesh N, Geha RS. Erk/Src phosphorylation of cortactin acts as a switch on-switch off mechanism that controls its ability to activate N-WASP. Mol Cell Biol 2004; 24:5269-80.

12. Kruchten AE, Krueger EW, Wang Y, McNiven MA. Distinct phospho-forms of cortactin differentially regulate actin polymerization and focal adhesions. Am J Physiol Cell Physiol 2008; 295:1113-22.
13. Ayala I, Baldassarre M, Giacchetti G, Caldieri G, Tetè S, Luini A, et al. Multiple regulatory inputs converge on cortactin to control invadopodia biogenesis and extracellular matrix degradation. J Cell Sci 2008 ; 121:369-78.

14. Tehrani S, Tomasevic N, Weed S, Sakowicz R, Cooper JA. Src phosphorylation of cortactin enhances actin assembly. Proc Natl Acad Sci USA 2007; 104:11933-8.

15. Oser M, Yamaguchi H, Mader CC, Bravo-Cordero JJ, Arias $\mathrm{M}$, Chen $\mathrm{X}$, et al. Cortactin regulates cofilin and N-WASp activities to control the stages of
invadopodium assembly and maturation. J Cell Biol invadopodium asse

16. Kinley AW, Weed SA, Weaver AM, Karginov AV, Bissonette E, Cooper JA, et al. Cortactin interacts with WIP in regulating Arp 2/3 activation and membrane protrusion. Curr Biol 2003; 13:384-93.

17. Hou P, Estrada L, Kinley AW, Parsons JT, Vojtek $\mathrm{AB}$, Gorski JL. Fgdl, the Cdc42 GEF responsible for Faciogenital Dysplasia, directly interacts with cortactin and $\mathrm{mAbpl}$ to modulate cell shape. Hum Mol Genet 2003; 12:1981-93.

18. Schafer DA, Weed SA, Binns D, Karginov AV, Parsons JT, Cooper JA. Dynamin2 and cortactin regulate actin assembly and filament organization. Curr Biol 2002; 12:1852-7.

19. Dudek SM, Birukov KG, Zhan X, Garcia JG. Novel interaction of cortactin with endothelial cell myosin light chain kinase. Biochem Biophys Res Commun 2002; 298:511-9.

20. Le Clainche C, Pauly BS, Zhang CX, EngqvistGoldstein AE, Cunningham K, Drubin DG. A HiplR-cortactin complex negatively regulates actin assembly associated with endocytosis. EMBO J 2007, 26:1199-210.

21. Ammer AG, Kelley LC, Hayes KE, Evans JV, LopezSkinner LA, Martin KH, et al. Saracatinib impairs head and neck squamous cell carcinoma invasion by disrupting invadopodia function. J Cancer Sci Ther 2009; 1:52-61.

22. Cao H, Chen J, Krueger EW, McNiven MA. SRCmediated phosphorylation of dynamin and cortactin regulates the "constitutive" endocytosis of transferrin. Mol Cell Biol 2010; 30:781-92

23. Zhu J, Yu D, Zeng XC, Zhou K, Zhan X. Receptormediated endocytosis involves tyrosine phosphorylation of cortactin. J Biol Chem 2007; 282:16086-94.

24. Grassart A, Meas-Yedid V, Dufour A, Olivo-Marin JC, Dautry-Varsat A, Sauvonnet N. Pakl phosphory. lation enhances cortactin-N-WASP interaction in clathrin-caveolin-independent endocytosis. Traffic 2010; 11:1079-91. 


\section{Further insights into cortactin conformational regulation}

Jason V. Evans, Laura C. Kelley, Karen E. Hayes, Amanda Gatesman Ammer, Karen H. Martin and Scott A. Weed* Department of Neurobiology and Anatomy; Program in Cancer Cell Biology; Mary Babb Randolph Cancer Center; West Virginia University; Morgantown, WV USA

Key words: cortactin, conformation, ERK1/2, Src, N-WASp, phosphorylation, circular dichroism, motility, invasion

Submitted: 12/06/10

Accepted: 12/21/10

DOI: 10.4161/bioa.1.1.14631

*Correspondence to: Scott A. Weed; Email: sweed@hsc.wvu.edu

Perspective on: Kelley LC, Hayes KE, Ammer AG, Martin KH, Weed SA. Cortactin phosphorylated by ERK1/2 localizes to sites of dynamic actin regulation and is required for carcinoma lamellipodia persistence. PLoS One 2010; 5:e13847. PMID: 21079800; DOl: 10.1371/journal. pone.0013847.
$\mathrm{T}$ he actin regulatory protein cortactin is involved in multiple signaling pathways impinging on the cortical actin cytoskeleton. Cortactin is phosphorylated by ERK1/2 and Src family tyrosine kinases, resulting in neuronal Wiskott Aldrich Syndrome protein (N-WASp) activation and enhanced actin related protein (Arp)2/3-mediated actin nucleation. Cortactin migrates as an $80 / 85$ $\mathrm{kDa}$ doublet when analyzed by SDSPAGE. Phosphorylation by ERK1/2 is associated with conversion of the $80 \mathrm{kDa}$ to the $85 \mathrm{kDa}$ form, postulated to occur by inducing a conformational alteration that releases the carboxyl-terminal $\mathrm{SH} 3$ domain from autoinhibition. Our recent analysis of the $80-85 \mathrm{kDa}$ cortactin "shift" in tumor cells indicates that while ERK1/2 phosphorylation is associated with the $85 \mathrm{kDa}$ shift, this phosphorylation event is not required for the shift to occur, nor does ERK1/2 phosphorylation appreciably alter global cortactin confirmation. These data indicate that additional factors besides ERK $1 / 2$ phosphorylation contribute to generating and/or maintaining the activated 85 $\mathrm{kDa}$ cortactin form in stimulated cells.

Changes in protein conformation are important for generating and propagating intracellular signal transduction events. During normal and pathogenic cell motility, regulation of actin cytoskeletal dynamics responsible for generating movement is dependent on conformational alterations in protein relay systems that activate and terminate signaling pathways responsible for initiating and maintaining motility. Proteins at the intersection of this molecular circuitry are key mediators in motility-driven signal regulation. The filamentous (F)-actin binding protein cortactin interacts with Arp2/3 complex to stimulate and stabilize Arp2/3-F-actin networks in lamellipodia and invadopodia of motile and invasive tumor cells. ${ }^{1,2}$ Cortactin is also a substrate for multiple protein kinases; most notably by ERK1/2 at S405 and $\mathrm{S} 418,{ }^{3}$ and Src family tyrosine kinases at Y421, Y470 and Y486. ${ }^{2}$ The ERK1/2 and Src phosphorylation sites all reside within a proline-rich region adjacent to a $\mathrm{SH} 3$ domain at the extreme carboxyl terminus. ${ }^{2,3}$ Phosphorylation of cortactin by ERK1/2 and tyrosine kinases promotes tumor cell migration and is required for invadopodia-mediated extracellular matrix degradation activity, ${ }^{4,5}$ demonstrating important functional roles for these phosphorylation events in cancer progression.

At the mechanistic level, cortactin tyrosine phosphorylation stimulates pro-invasive activity by providing binding sites at Y421 and Y466 for the SH2 domain of the adaptor protein NCK1. ${ }^{6.8}$ NCK1, through its $\mathrm{SH} 3$ domain, binds N-WASp to release an acidic carboxylterminal (VCA) domain that is responsible for binding to and activating Arp2/3 complex, promoting actin polymerization. ${ }^{9,10}$ In the case of ERK1/2, cortactin phosphorylation at $\mathrm{S} 405 / 418$ promotes association of the carboxyl-terminal $\mathrm{SH} 3$ domain with N-WASp, resulting in a similar Arp2/3 activation cascade. ${ }^{11}$

Cortactin purified from most cell types as well as from recombinant cell-free sources typically migrates as an $80 / 85 \mathrm{kDa}$ doublet in SDS-PAGE gels. ${ }^{12-14}$ This doublet most likely represents a single polypeptide, since electrophoresis in urea-containing 


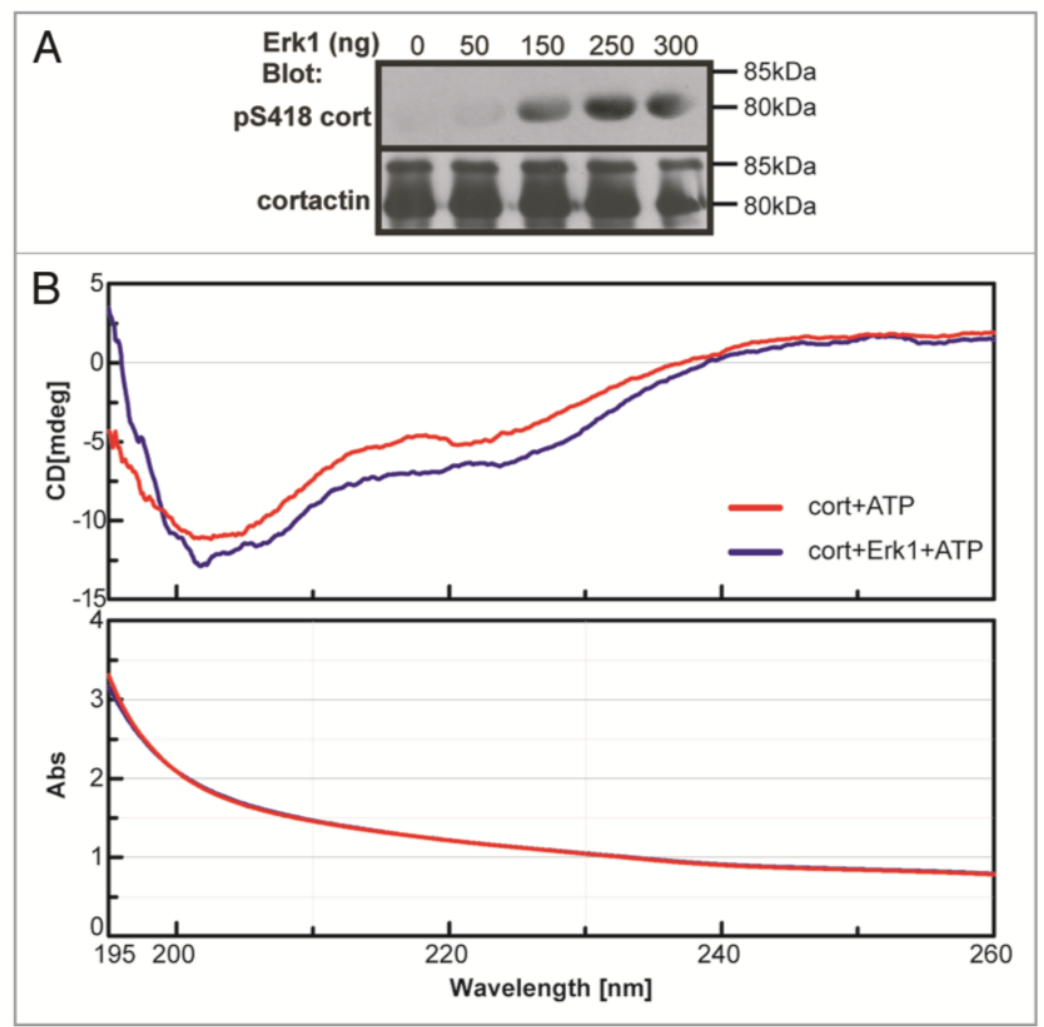

Figure 1. Analysis of cortactin secondary structure following ERK1 phosphorylation. (A) Phosphorylation of cortactin by ERK1. Recombinant cortactin $(25 \mu \mathrm{g})$ was phosphorylated with the indicated amounts of ERK 1 at $30^{\circ} \mathrm{C}$ for $30 \mathrm{~min}$. Aliquots of each reaction were analyzed by western blotting with anti-pS418 and total cortactin antibodies. The positions of the $80 \mathrm{kDa}$ and $85 \mathrm{kDa}$ forms are denoted. (B) Kinase reactions containing recombinant cortactin $(25 \mu \mathrm{g})$ and ATP $(500 \mu \mathrm{M})$ incubated without (red line) or with (blue line) $250 \mathrm{ng}$ ERK1 were analyzed with a Jasco J-810 Spectropolarimeter using a scan speed of $50 \mathrm{~nm} / \mathrm{min}$ with a $4 \mathrm{sec}$ response time, $1 \mathrm{~nm}$ band width and $0.1 \mathrm{~nm}$ data pitch (top). The absorbance for each reaction was monitored throughout the analyzed wavelength range (bottom) to control for sample-to-sample variability. Scans are shown for each reaction condition following rendering with Spectra Manager software ( $v$ 1.53.01).

gels results in the sole presence of the 85 $\mathrm{kDa}$ form, ${ }^{14}$ supporting the notion that the $80 \mathrm{kDa}$ and $85 \mathrm{kDa}$ cortactin bands represent different conformational isomers. Phosphorylation of cortactin downstream of epidermal growth factor receptor (EGFR) activation results in a shift from 80 $\mathrm{kDa}$ to $85 \mathrm{kDa}$. Under these conditions the $85 \mathrm{kDa}$ cortactin form displays increased serine and threonine phosphorylation, ${ }^{15}$ with the "shift" in cortactin $M_{\mathrm{r}}$ occurring concurrently with ERK1/2-mediated S405 and S418 phosphorylation. ${ }^{3}$ Collectively these reports have resulted in proposing that non-phosphorylated cortactin exists in the $80 \mathrm{kDa}$ "closed" form with the carboxyl-terminal $\mathrm{SH} 3$ domain binding back upon the proline-rich cortactin domain, blocking the ability of the $\mathrm{SH} 3$ domain binding interface to interact with other ligands. ERK1/2 phosphorylation in turn results in displacing the $\mathrm{SH} 3$-proline-rich homotypic cortactin interaction, rendering cortactin in an "open" $85 \mathrm{kDa}$ state where the $\mathrm{SH} 3$ domain can bind N-WASp and other cortactin $\mathrm{SH} 3$ binding proteins. $3,11,16$ These conclusions are supported by chemical crosslinking studies with non-phosphorylated cortactin that indicate cortactin exists as a monomeric globular protein in solution, with the $\mathrm{SH} 3$ domain in close proximity to a helical domain amino terminal to the proline-rich region ${ }^{17}$ as well as the prevalence of the $85 \mathrm{kDa}$ form in metastatic colorectal carcinoma cases. ${ }^{18}$

We recently analyzed the cortactin "shift" through a combination of phosphorylation-specific antibodies against cortactin pS405/pS418 and point mutant constructs for these residues. Phosphorylation-specific antibodies confirm the presence of pS405 and pS418 predominantly in the $85 \mathrm{kDa}$ cortactin form in tumor cells following EGF treatment. However, analysis of the cortactin shift with phosphorylation-null point mutants downstream of $\mathrm{v}$-Src mediated ERK1/2 activation reveals a more intricate result. Cortactin S418A and S405A/ S418A proteins exhibit the same electrophoretic mobility as wild-type cortactin, with the prominent band at $80 \mathrm{kDa}$, while a $5405 \mathrm{~A}$ cortactin mutant runs at the shifted $85 \mathrm{kDa} M_{\mathrm{r}}$. This could suggest that S418 phosphorylation alone is responsible for driving the $80 / 85 \mathrm{kDa}$ cortactin shift, with S405 phosphorylation occurring 
subsequent to $\$ 418$ phosphorylation in the $85 \mathrm{kDa}$ form. However, cortactin phosphorylated by ERK1 at $\mathrm{pS} 418$ in vitro does not shift from $80 \mathrm{kDa}$ to $85 \mathrm{kDa}$ (Fig. 1A) and ERK1-phosphorylated cortactin does not demonstrate significant alterations in secondary structure compared to nonphosphorylated cortactin when evaluated by circular dichroism (Fig. 1B). These results demonstrate that ERK-mediated cortactin phosphorylation on S405 and S418 is associated with, but is not exclusively responsible for production of the $85 \mathrm{kDa}$ open cortactin form.

We conclude that other factors besides S405/418 phosphorylation are involved in generating and/or maintaining the cortactin shift in EGF-stimulated cells. While the identity of these additional regulatory elements is presently unclear, the prolinerich region where serine 405 and serine 418 reside allows ample opportunity for substantial global conformational alterations through cis-trans isomerization of proline peptide bonds by prolyl isomerases. ${ }^{19}$ The compact globular conformation (presumably representative of the closed $80 \mathrm{kDa}$ isomer), with the $\mathrm{SH} 3$ domain folding back onto amino-terminal peptide sequences assumed by cortactin in solution ${ }^{17}$ is in contrast to the 220 $\AA$-long rod-like cortactin protein characterized by rotary shadowing and electron microscopy ${ }^{20}$ that may represent the 85 $\mathrm{kDa}$ form. These studies provide indirect evidence for involvement of the prolinerich region in extensively altering cortactin structure.

\section{Acknowledgements}

We thank P. Gannett and J. Jett for assistance with circular dichroism work and data analysis. This work was supported by a subproject of NIH grant P20-RR16440 to S.A.W.

1. Weaver AM. Cortactin in tumor invasiveness. Cance Lett 2008; 265:157-66.

2. Ammer AG, Weed SA. Cortactin branches out: roles in regulating protrusive actin dynamics. Cell Motil Cytoskeleton 2008; 65:687-707.

3. Campbell DH, Sutherland RL, Daly RJ. Signaling pathways and structural domains required for phosphorylation of EMS1/cortactin. Cancer Res 1999; 59:5376-85.

4. Kruchten AE, Krueger EW, Wang Y, McNiven MA Distinct phospho-forms of cortactin differentially regulate actin polymerization and focal adhesions. Am J Physiol Cell Physiol 2008; 295:1113-22.

5. Ayala I, Baldassarre M, Giacchetti G, et al. Multiple regulatory inputs converge on cortactin to control invadopodia biogenesis and extracellular matrix deg. radation. J Cell Sci 2008; 121:369-78.

6. Tehrani S, Tomasevic N, Weed S, Sakowicz R, Cooper IA. Src phosphorylation of cortactin enhances actin assembly. Proc Natl Acad Sci USA 2007; 104:11933-8.

7. Oser M, Yamaguchi H, Mader CC, et al. Cortactin regulates cofilin and N-WASp activities to control the stages of invadopodium assembly and maturation. J Cell Biol 2009; 186:571-87.

8. Oser M, Mader CC, Gil-Henn H, et al. Specific tyrosine phosphorylation sites on cortactin regulate Nckl-dependent actin polymerization in invadopodia. I Cell Sci 2010; 123:3662-73.

9. Tomasevic N, Jia Z, Russell A, et al. Differential regulation of WASP and N-WASP by Cdc42, Racl, Nck and $\mathrm{PI}(4,5) \mathrm{P} 2$. Biochemistry 2007; 46:3494-502.
10. Padrick SB, Rosen MK. Physical mechanisms of signal integration by WASP family proteins. Annu Rev Biochem 2010; 79:707-35.

11. Martinez-Quiles N, Ho HY, Kirschner MW, Ramesh N, Geha RS. Erk/Src phosphorylation of cortactin acts as a switch on-switch off mechanism that controls its ability to activate N-WASP. Mol Cell Biol 2004: 24:5269-80

12. Wu H, Reynolds AB, Kanner SB, Vines RR, Parsons JT. Identification and characterization of a novel cytoskeleton-associated pp60src substrate. Mol Cell Biol 1991; 11:5113-24.

13. Wu H, Parsons JT. Cortactin, an 80/85 kilodalton pp60src substrate, is a filamentous actin-binding protein enriched in the cell cortex. J Cell Biol 1993; 120:1417-26.

14. Huang C, Ni Y, Wang T, Gao Y, Haudenschild CC, Zhan X. Downregulation of the filamentous actin cross-linking activity of cortactin by Src-mediated tyrosine phosphorylation. J Biol Chem 1997; 272:13911-5.

15. van Damme H, Brok H, Schuuring-Scholtes E, Schuuring E. The redistribution of cortactin into cell-matrix contact sites in human carcinoma cells with 11q13 amplification is associated with both overexpression and post-translational modification. J Biol Chem 1997; 272:7374-80.

16. Lua BL, Low BC. Cortactin phosphorylation as a switch for actin cytoskeletal network and cell dynamics control. FEBS Lett 2005; 579:577-85.

17. Cowieson NP, King G, Cookson D, et al. Cortactin adopts a globular conformation and bundles actin into sheets. J Biol Chem 2008; 283:16187-93.

18. Zhang LH, Tian B, Diao LR, et al. Dominant expression of $85 \mathrm{kDa}$ form of cortactin in colorectal cancer. J Cancer Res Clin Oncol 2006; 132:113-20.

19. Schiene C, Fischer G. Enzymes that catalyse the restructuring of proteins. Curr Opin Struct Biol $2000 ; 10: 40-5$.

20. Weaver AM, Heuser JE, Karginov AV, Lee WL, Parsons JT, Cooper JA. Interaction of cortactin and N-WASp with Arp2/3 complex. Curr Biol 2002; $12: 1270-8$ 


\title{
Oncogenic Src requires a wild-type counterpart to regulate invadopodia maturation
}

\author{
Laura C. Kelley ${ }^{1}$, Amanda Gatesman Ammer ${ }^{1}$, Karen E. Hayes ${ }^{1}$, Karen H. Martin ${ }^{1}$, Kazuya Machida ${ }^{2}$, Lin Jia ${ }^{2}$, \\ Bruce J. Mayer ${ }^{2}$ and Scott A. Weed ${ }^{1, *}$ \\ ${ }^{1}$ Department of Neurobiology and Anatomy, Program in Cancer Cell Biology, Mary Babb Randolph Cancer Center, West Virginia University, \\ Morgantown, West Virginia, 26506-9300, USA \\ ${ }^{2}$ Raymond and Beverly Sackler Laboratory of Genetics and Molecular Medicine, Department of Genetics and Developmental Biology, \\ University of Connecticut Health Center, Farmington, CT 06030, USA \\ "Author for correspondence (sweed @hsc.wvu.edu) \\ Accepted 27 July 2010 \\ Journal of Cell Science 123, 3923-3932 \\ 2010. Published by The Company of Biologists Ltd \\ doi: $10.1242 /$ ics. 075200
}

\section{Summary}

The proto-oncogene Src tyrosine kinase (Src) is overexpressed in human cancers and is currently a target of anti-invasive therapies. Activation of Src is an essential catalyst of invadopodia production. Invadopodia are cellular structures that mediate extracellular matrix $(\mathrm{ECM})$ proteolysis, allowing invasive cell types to breach confining tissue barriers. Invadopodia assembly and maturation is a multistep process, first requiring the targeting of actin-associated proteins to form pre-invadopodia, which subsequently mature by recruitment and activation of matrix metalloproteases (MMPs) that facilitate ECM degradation. We demonstrate that active, oncogenic Src alleles require the presence of a wild-type counterpart to induce ECM degradation at invadopodia sites. In addition, we identify the phosphorylation of the invadopodia regulatory protein cortactin as an important mediator of invadopodia maturation downstream of wild-type Src. Distinct phosphotyrosine-based protein-binding profiles in cells forming pre-invadopodia and mature invadopodia were identified by SH2-domain array analysis. These results indicate that although elevated Src kinase activity is required to target actin-associated proteins to pre-invadopodia, regulated Src activity is required for invadopodia maturation and matrix degradation activity. Our findings describe a previously unappreciated role for proto-oncogenic Src in enabling the invasive activity of constitutively active Src alleles.

Key words: Head and Neck cancer, Src, Invadopodia, Cortactin

\section{Introduction}

Src is the first described proto-oncogene and a current target for anti-invasive compounds in clinical trials (Brunton and Frame, 2008; Yeatman, 2004). Src participates in a vast array of cellular functions that include the regulation of cell proliferation, adhesion, migration and invasion (Guarino, 2010; Thomas and Brugge, 1997). In normal cells, Src activity is tightly controlled through intramolecular regulation, subcellular localization and protein expression levels. Elevated or aberrant Src activity is a potent mediator of cell transformation and tumor progression, and is associated with the majority of human cancers including head and neck squamous cell carcinoma (HNSCC) (Summy and Gallick, 2003).

One of the most evident phenotypes of Src-transformed cells is the formation of actin-rich ventral membrane protrusive structures that actively degrade ECM (Chen, 1989). These structures, termed invadopodia, are made by metastatic cancer cells and Srctransformed fibroblasts (Linder, 2009). Invadopodia spontaneously form in tumor cells directly cultured from patient samples and are hypothesized to facilitate breaching of basement membranes during metastasis (Clark et al., 2007; Yamaguchi and Condeelis, 2007). Src activity is absolutely necessary for invadopodia formation and function, and the level of tyrosine phosphorylation at invadopodia positively correlates with the degree of ECM degradation (Bowden et al., 2006; Spinardi et al., 2004). The molecular components that make up invadopodia include proteins that facilitate actin assembly, membrane trafficking and focal degradation. Src substrates participate in all of these functions and include the proteins cortactin
(Bowden et al., 1999), N-WASp (Yamaguchi et al., 2005), dynamin2 (Baldassarre et al., 2003), AMAP1 (Onodera et al., 2005), paxillin (Bowden et al., 1999), p130Cas (Brabek et al., 2004), Tsk5 (Seals et al., 2005), p190RhoGAP (Nakahara et al., 1998), AFAP110 (Gatesman et al., 2004) and caveolin (Yamaguchi et al., 2009). Several studies have evaluated Src activity in invadopodia formation through the ectopic expression of constitutively active Src alleles (Artym et al., 2006; Oser et al., 2009; Stylli et al., 2009). However, these activating Src mutants are rarely found in human tumors, which instead typically contain increased levels of wild-type (WT) Src expression and/or aberrant WT Src activity due to hyperactivation of upstream pathways (Yeatman, 2004). The role of WT Src in invadopodia formation and function is unknown.

Invadopodia assembly has been proposed to involve several stages that regulate the progression from pre-invadopodia (nondegradative) complexes to functional, mature invadopodia containing active MMPs that degrade ECM (Artym et al., 2006; Oser et al., 2009). In current models of invadopodia formation, filamentous (F)-actin and the actin-associated protein cortactin are recruited to sites of matrix adhesion, resulting in pre-invadopodia complexes (Artym et al., 2006). The membrane-bound matrix metalloproteinase MT1-MMP (MMP14) is subsequently recruited to these sites, allowing matrix degradation and invadopodia maturation. Cortactin is an actin-binding protein that is phosphorylated by Src kinase (Head et al., 2003), and is a core invadopodia component. Knockdown of cortactin expression results in decreased invadopodia formation (Artym et al., 2006; Webb et 
al., 2007) and MMP secretion (Clark and Weaver, 2008; Clark et al., 2007), whereas phosphorylation of cortactin is important for regulation of matrix degradation at invadopodia (Ayala et al., 2008).

Recent work on discerning the molecular mechanism regulating actin polymerization before MMP recruitment has identified dynamic regulation of cortactin phosphorylation or dephophorylation downstream of Src to be central to this process (Oser et al., 2009). In this model, cortactin sequesters the actinsevering protein cofilin within pre-invadopodia. Tyrosine phosphorylation of cortactin releases cofilin, which in turn accelerates actin polymerization by severing existing invadopodial actin filaments. In addition, tyrosine phosphorylation of cortactin creates docking sites for the adaptor protein Nck1, which binds and activates the Arp2 $/ 3$ activator N-WASp. The combined effect of cofilin activation and N-WASp-mediated Arp2/3 activity serves to increase actin polymerization as pre-invadopodia mature and obtain the ability to degrade ECM. Dephosphorylation of cortactin is proposed to stabilize maturing invadopodia by downregulating actin polymerization through liberation of the Nck1-N-WASp complex, coupled with rebinding of inactive cofilin. Although these data implicate cycles of cortactin phosphorylation and dephosphorylation as crucial in invadopodia maturation, it is not known how the phosphorylation of cortactin and other invadopodia maturation-associated proteins is spatially and temporally orchestrated through upstream kinase-based signaling to drive invadopodia maturation. In this study, we have determined that the presence of endogenous, regulated WT c-Src is required for the maturation of pre-invadopodia complexes induced by oncogenic Src activity into degradative invadopodia. In addition, we show that cortactin phosphorylation downstream of WT c-Src is an important mediator of the maturation process.

\section{Results}

Elevated Src activity regulates invadopodia formation in HNSCC cell lines

The introduction of constitutively active viral Src (v-Src) or constitutively active cellular Src (Src527F) has been examined in invadopodia formation in cancer cell lines (Artym et al., 2006; Buschman et al., 2009; Oikawa et al., 2008; Stylli et al., 2009), but the role of endogenous c-Src in invadopodia function is unclear. We analyzed a panel of HNSCC lines for endogenous c-Src activity and total c-Src protein levels (Fig. 1A), as well as the ability of these lines to form spontaneous invadopodia on FITC-gelatin matrix (Fig. 1B; supplementary material Fig. S1A). Two (UMSCC1 and OSC19) out of the six tested lines generate invadopodia that were identified by the colocalization of actin and cortactin-rich yellow aggregates in merged images (Fig. 1B, white arrows) coinciding with areas of focal gelatin degradation (black arrows). UMSCC1 and OSC19 cells had substantially elevated $\mathrm{c}$-Src expression and c-Src activity compared with the UMSCC2, 1483 and MSK921 cell lines that do not make invadopodia (Fig. 1A). The FADU cells had elevated c-Src activity, but failed to generate spontaneous invadopodia, suggesting that elevated Src expression alone is not sufficient to drive invadopodia biogenesis in this line. However, invadopodia are formed and matrix degradation occurs in all the HNSCC cell lines that do not form spontaneous invadopodia (UMSCC2, 1483, FADU and MSK921) following exogenous Src527F expression (Fig. 1B; supplementary material Fig. S1B). These data suggest a cell-linespecific threshold of Src activity (highest in FADU cells) that must be reached to support invadopodia formation and matrix degradation. These results are consistent with the idea that elevated Src activity drives invadopodia biogenesis, and are in agreement with previous work with small-molecule Src inhibitors in HNSCC cell lines, which found a dose-dependent decrease of invadopodia formation and matrix degradation (Ammer et al., 2009). Similar results have also been shown for breast cancer cells (Pichot et al., 2009).

\section{Endogenous Src expression is required for efficient invadopodia-based matrix degradation in HNSCC cells expressing constitutively active Src}

To test the effect of depletion of endogenous c-Src on HNSCC invadopodia formation and function, c-Src expression was knocked down in UMSCC1 cells by RNA interference (SrcSi) (Fig. 2A) and assayed for invadopodia formation and gelatin degradation (Fig. 2B). Endogenous c-Src was depleted by $58 \%$ at 2 days, and by $70 \%$ at 3 days after transfection. Src-knockdown cells had no statistically significant difference in the number of cells with invadopodia or the number of invadopodia per cell (Fig. 2C, top and middle panels). However, $\mathrm{SrcSi}$ cells exhibited a $57 \%$ decrease
A

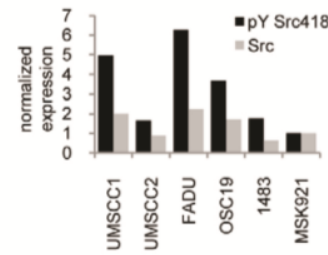

B

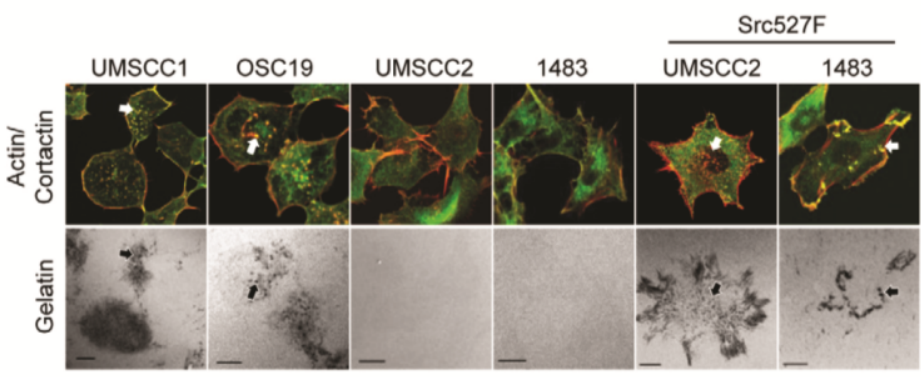

Fig. 1. Sre activity regulates invadopodia formation in HNSCC lines. (A) Protein levels of active c-Src (Src-pY ${ }^{418}$ ), total c-Src (Src) and $\beta$-actin (loading control) in HNSCC lines. For quantification of active Src and total Src, expression levels were normalized to MSK921 cells, a line with low Src activity that does not form invadopodia. The multiple bands in the Src-p $\mathrm{Y}^{418}$ panel presumably represent additional Src Family kinases; see Fig. 3A. (B) HNSCC cell lines with or without Src-527F were incubated on FITC-gelatin (pseudocolored white) coverslips for 12 hours and labeled with TRITC-phalloidin (red) and anti-cortactin (green). Invadopodia are identified by the yellow aggregates in the merged images of actin and cortactin (white arrows) that localize with the dark holes in the FITC-gelatin (black arrows). Scale bars: $10 \mu \mathrm{m}$. 
A

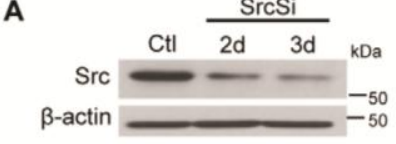

B

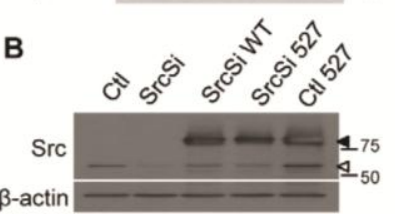

C

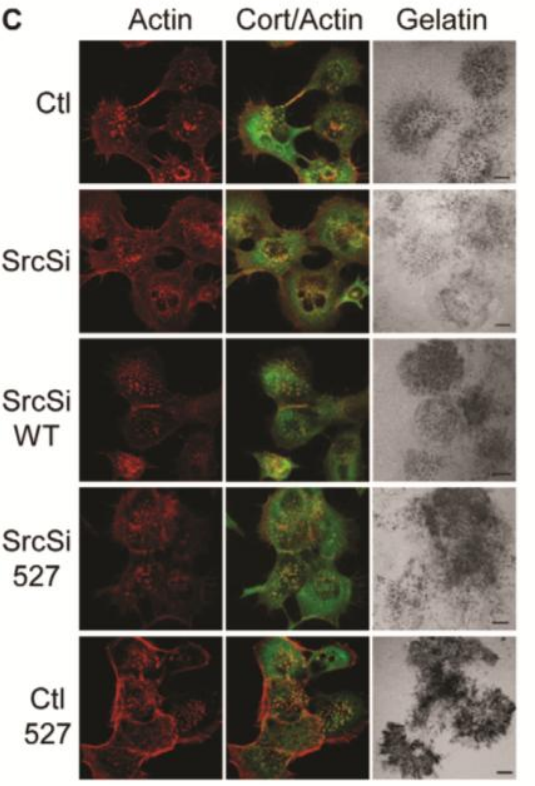

D
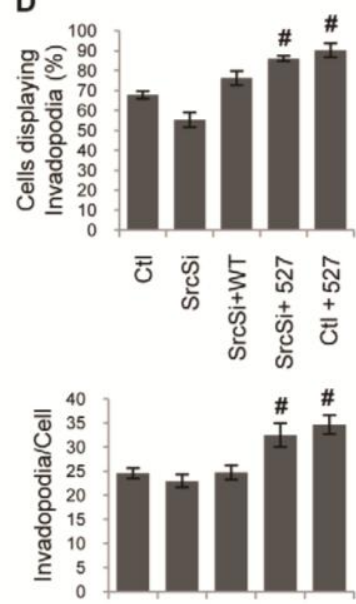

ฮ
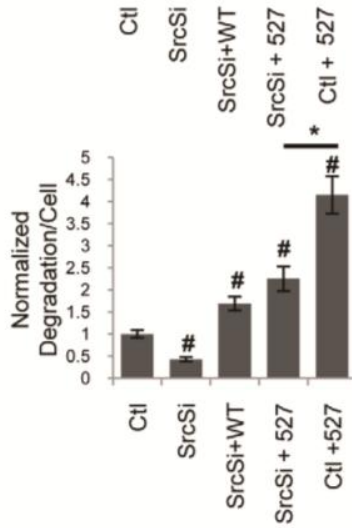

Fig. 2. Inhibition of endogenous Src expression decreases matrix degradation independently of changes in invadopodia number or increased Sre activity. (A) UMSCC1 cells transfected with siRNA directed against $\mathrm{Src}(\mathrm{SrcSi})$ or a non-targeting siRNA $(\mathrm{Ctl})$ were evaluated for Src knockdown 2 and 3 days after transfection by anti-Src western blotting. $\beta$-actin blotting was used as a loading control. (B) Cell lysates from UMSCC1 cells transfected with non-targeting siRNA (Ctl) or SRC siRNA ( $\mathrm{SrcSi}$ ) alone, or in combination with cerulean-tagged SrcWT (WT) or Src-527F (527) were evaluated by immunoblotting. Lysates were probed with anti-Src and anti- $\beta$-actin antibodies. Filled arrowhead indicates exogenously expressed Src (WT or 527F), open arrowhead denotes endogenous Src. (C) Representative confocal images of UMSCC1 cells transfected with nontargeting siRNA (Ctl) or SrcSi alone, or in combination with cerulean-tagged SrcWT (WT) or Src-527F (527). Cells were plated on FITC-gelatin-coated (pseudocolored white) coverslips for 10 hours and immunolabeled with TRITC-phalloidin (red) and cortactin (green). Scale bars: $10 \mu \mathrm{m}$. (D) Percentage of cells displaying invadopodia (top), the number of invadopodia per cell (middle) and the amount of matrix degradation per cell (bottom) were examined for each line evaluated in B. Data are presented as mean \pm s.e.m.; ", statistically different from control $(P \leq 0.01)$; groups under the bar are statistically different $(P<0.01)$. in gelatin degradation compared with control cells (Ctl) (Fig. 2C, bottom panel). Rescue of WT Src expression in SrcSi cells $(\mathrm{SrcSi}+\mathrm{WT})$ restored matrix degradation to levels above those of the control cells (1.7-fold) (Fig. 2C). Increases above control levels are presumably due to the additive effect of remaining endogenous Src from incomplete knockdown coupled with the modest overexpression of the WT Sre construct (Fig. 2B).

We also examined the effect of silencing endogenous $\mathrm{c}-\mathrm{Src}$ in the presence of constitutively active Src (Fig. 2B,C). Consistently with previous reports (Artym et al., 2006; Oser et al., 2009), expression of Src527F in control cells $(\mathrm{Ctl}+527)$ or SrcSi cells ( $\mathrm{SrcSi}+527 \mathrm{~F})$ resulted in increases in the number of cells with invadopodia $(\sim 30 \%)$ and the number invadopodia per cell $(\sim 36 \%)$. Surprisingly, c-Src knockdown markedly blunted the increased degradation due to Src527F expression, a 2.5-fold (SrcSi+Src527F cells) increase compared with a 4.8 -fold $(\mathrm{Ctl}+527 \mathrm{~F})$ increase over control cells (Fig. 2C). These results demonstrate that constitutively active Src cannot completely rescue the role of endogenous c-Src in regulation of matrix degradation. Collectively, these data indicate that although increased Src activity enhances invadopodia formation, the presence of endogenous c-Src is required for optimal matrix degradation. Similar results were found in OSC19 cells (supplementary material Fig. S2).
Constitutively active Src is sufficient to promote invadopodia formation but not ECM degradation in Srcnull cells

Complete c-Src knockdown in our HNSCC lines is technically problematic because additional Src family kinases (Yes and Fyn) with unknown functions in invadopodia biology are present and maintained in OSC19 and UMSCC1 cell lines treated with SrcSi (Fig. 3A). We therefore used Src-, Yes- and Fyn-deficient (SYF) fibroblasts to further evaluate the role of WT Src in invadopodia function. A GFP-tagged temperature-sensitive mutant of $\mathrm{v}$-Src (tsLa29-GFP) was generated and expressed in SYF cells to dynamically regulate Src activity and invadopodia formation. Src kinase activation occurs within 15 minutes when cells are switched from the non-permissive temperature $\left(41^{\circ} \mathrm{C}\right)$ to the permissive temperature $\left(35^{\circ} \mathrm{C}\right)$ (Fig. 3B). v-Src inactivation occurs within 30 minutes when cells are shifted back to $41^{\circ} \mathrm{C}$. Activation of v-Src leads to the phosphorylation of cortactin on Tyr421, indicating that tsLa29-GFP regulates phosphorylation of a known downstream Src target that is crucial for invadopodia assembly. In agreement with previous reports (Walker et al., 2007), invadopodia formation is induced when cells expressing tsLa29-GFP are switched to the permissive temperature (Fig. 3C). These invadopodia are enriched with active $\mathrm{V}-\mathrm{Src}$ and phosphorylated cortactin (Fig. 3C; 

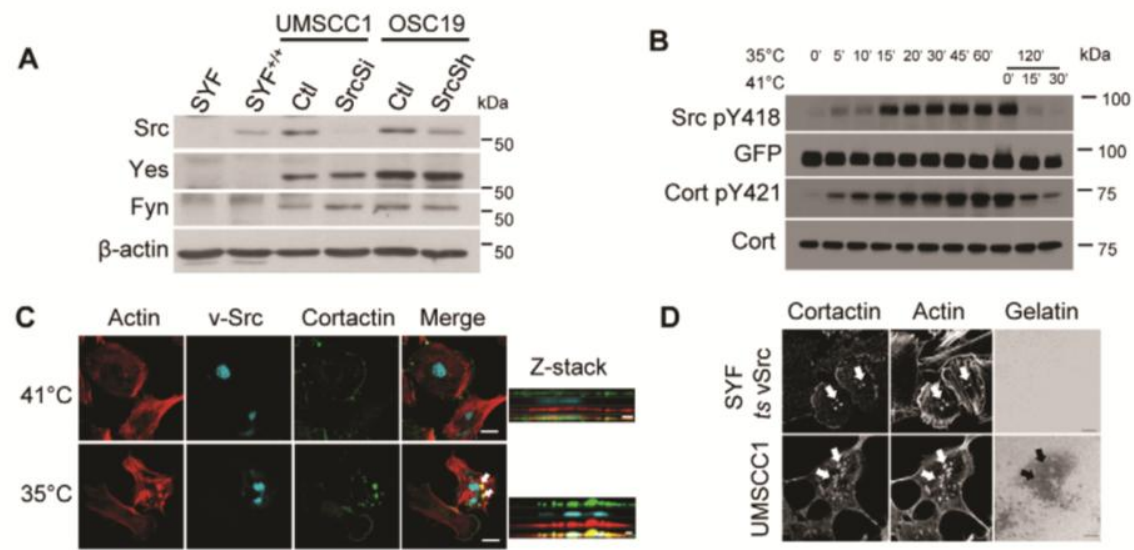

Fig. 3. Invadopodia produced by constitutively active Src in SYF fibroblasts fail to degrade ECM. (A) Src family kinase expression in HNSCC and fibroblast cell lines. Clarified cell lysates from SYF, SYF ${ }^{+/+}$, UMSCC1 (treated with control and SrcSi), and OSC19 (expressing control vector and SRC shRNA) cells were resolved by SDS-PAGE and immunoblotted with anti-Src, anti-Yes, anti-Fyn and anti- $\beta$-actin antibodies. (B) Time course of tsLa29 v-Src activation and resulting cortactin phosphorylation. Cells transfected with tsLa29 were incubated at $35^{\circ} \mathrm{C}$ (permissive temperature) for the indicated times and were lysed or returned to $41^{\circ} \mathrm{C}$ (non-permissive temperature) for 15 or 30 minutes and analyzed for Src-p $\mathrm{Y}^{418}$, GFP, cortactin, and cortactin-pY $\mathrm{Y}^{421}$. (C) Invadopodia formation in cells expressing v-Src. SYF cells were transfected with tsLa29-GFP (pseudocolored light blue) and incubated at $41^{\circ} \mathrm{C}$ or $35^{\circ} \mathrm{C}$ and labeled with TRITC-phalloidin (red) and cortactin (green). Cells were visualized by confocal microscopy and $z$-stack sectioning. Invadopodia are visible in $z$-stack images as actin- and cortactin-rich puncta that are several micrometers in length (white arrows). Scale bars: $10 \mu \mathrm{m}, 5 \mu \mathrm{m}$ ( $z$-stacks). (D) Defective ECM degradation in SYF cells expressing v-Src. SYF cells transfected with tsLa29-v-Src and non-transfected UMSCC1 cells (positive control) were plated directly onto FITC-gelatin-coated coverslips. After 24 hours, cells were labeled with TRITC-phalloidin and anti-cortactin antibodies. White arrows indicate invadopodia and black arrows, areas of degraded matrix in C and D. Scale bars: $10 \mu \mathrm{m}$.

supplementary material Fig. S3A). As reported in other invadopodia-forming cell systems (Artym et al., 2006; Ayala et al., 2008; Clark et al., 2007; Webb et al., 2006), inhibition of cortactin expression using siRNA diminishes the ability of tsLa29-GFP to induce invadopodia formation at the permissive temperature (supplementary material Fig. S3B,C).

To evaluate the functionality of invadopodia in this system, we plated SYF cells on FITC-gelatin-coated coverslips to assay ECM degradation. Invadopodia induced by tsLa29-GFP in the Src-null fibroblasts fail to degrade the ECM at periods up to and beyond 48 hours, indicating they remain in a pre-invadopodia state (Fig. 3D). Experiments with an untagged v-Src produced a similar result, ruling out improper activation or localization related to the addition of GFP. We also attempted to rescue invadopodia maturation in the SYF cells with tsLa29 v-Src. Manipulation of tsLa29 v-Src activity over the 24 hour incubation period by switching cells from permissive to the non-permissive temperatures did not result in invadopodia maturation (data not shown). These results demonstrate that constitutively active $\mathrm{v}$-Src activity is responsible for the induction of the initial phosphorylation cascade that drives recruitment of invadopodia components to form pre-invadopodia complexes, but these v-Src-induced complexes are insufficient to direct matrix degradation in SYF cells.

\section{Wild-type Src kinase is necessary for invadopodia maturation}

Based on our findings we hypothesized that either: (1) another ubiquitously expressed Src family kinase (Yes and/or Fyn) absent from SYF cells is required for invadopodia maturation in addition to active Src, or (2) WT Src or 'regulated' Src must also be present with active Src for degradation of ECM to occur. To test these hypotheses, we used a SYF cell line with two copies of WT Src genetically reintroduced to restore normal WT Src expression $\left(\mathrm{SYF}^{+/+}\right.$; Fig. 3A, Fig. 4A). The percentage of cells forming invadopodia and the percentage of invadopodia-forming cells degrading matrix were assessed following introduction of $\mathrm{v}$-Src or Src527F. Transfection with activated Sre constructs in the form of tsLa29 or Src527F induces mature matrix-degrading invadopodia in $\mathrm{SYF}^{+/+}$cells, in contrast to pre-invadopodia formation in SYF cells (Fig. 4B). Approximately $55 \%$ of $\mathrm{SYF}^{+/+}$cells forming invadopodia contained invadopodia that actively degraded matrix, compared with $2 \%$ of SYF cells (Fig. 4C). There was no difference in the percentage of cells forming invadopodia (pre and mature) in SYF or SYF ${ }^{+/+}$cells expressing Src527F (Fig. 4C), which is similar to results in UMSCC1 cells (Fig. 2C). Also, the level of general phosphotyrosine-containing proteins localized to invadopodia was unchanged in SYF527F and SYF527F ${ }^{+/+}$cells (Fig. 4B). Taken together, these results suggest that catalytically active Src alone promotes the assembly of pre-invadopodia complexes and targets tyrosine phosphorylation of proteins within these structures, but SrcWT is necessary for pre-invadopodia maturation required to induce ECM degradation.

To confirm these findings, WT Src expression was transiently restored in SYF cells and assayed for ECM degradation. Monitoring of the coexpression of Src527F and SrcWT was achieved by creating C-terminal linker fusions with mCherry (Src527FmCherry) and cerulean (Src-Cer) fluorescent proteins (supplementary material Fig. S4). Transfection efficiency of the co-transfected Src constructs was consistently greater than $90 \%$ and imaging revealed that nearly all SYF cells expressed both Src alleles (supplementary material Fig. S5A). In SYF cells expressing SrcWT alone, Src had a perinuclear localization that was consistent with previous reports (Sandilands et al., 2004) (supplementary material Fig. S4B,C). Coexpression of Src527F with WT Src 
A

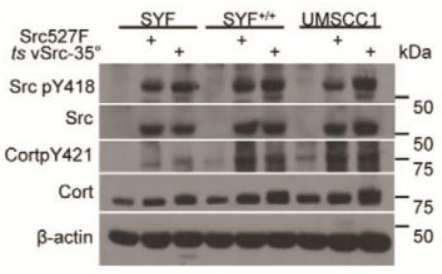

C

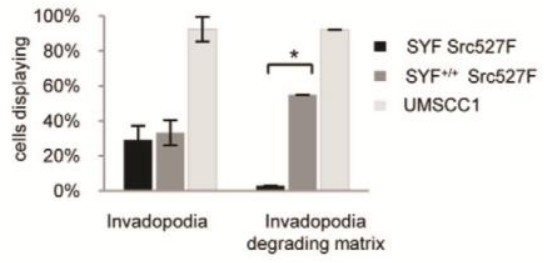

B

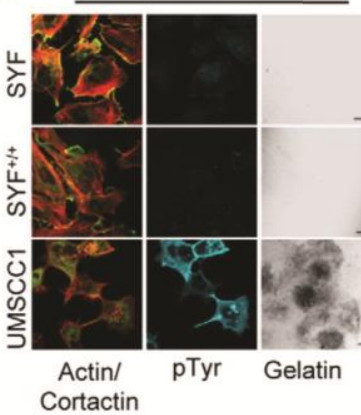

$\operatorname{Src527F}$
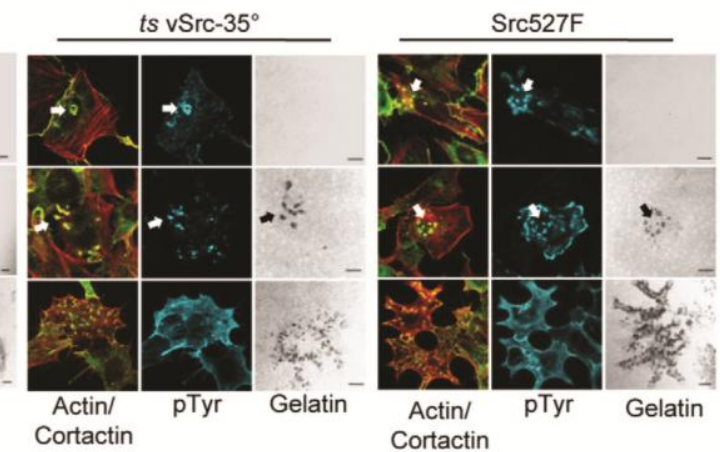

Fig. 4. WT Sre rescues invadopodia maturation in cells expressing constitutively active Src. (A) SYF, SYF $F^{+/+}$and UMSCC1 cells that were non-transfected (NT), transfected with Src-527F or ts-v-Src, were lysed, resolved by SDS-PAGE and immunoblotted with anti-Src-pY418, anti-Src clone EC10 (only recognizes avian Src), anti-cortactin-pY421, anti-cortactin (4F11), and anti- $\beta$-actin antibodies. (B) SYF, SYF ${ }^{t /+}$ and UMSCC1 cells that were non-transfected (NT), transfected with SRC-527F, or tsLa29-v-Src were plated onto FITC-conjugated gelatin coverslips and evaluated by confocal microscopy. Cells were labeled with TRITCphalloidin (red), cortactin (green), and anti-phosphotyrosine (light blue) antibodies. Invadopodia are identified by the yellow aggregates in the merged image of actin and cortactin in cells containing phosphotyrosine. In addition, degrading (mature) invadopodia localize with the dark holes (arrows) in the FITC-gelatin (white). (C) Quantification of the percentage of cells forming invadopodia structures (actin and cortactin aggregates, left), and the percentage of invadopodiaforming cells that contain matrix degradation (mature invadopodia, right). Data are represented as mean \pm s.d.; ${ }^{*} P \leq 0.05$.

results in recruitment of WT Src to invadopodia where it colocalizes with Src527F (Fig. 5A). In addition, cells coexpressing these constructs regain the ability to degrade ECM (Fig. 5B,C). To further verify the functional requirement for WT Src in invadopodia maturation, we conducted WT-Src-specific staining of SYF cells containing Src527F. WT Src colocalizes with cortactin to areas of ECM degradation, further demonstrating that Src localizes to mature invadopodia (Fig. 5D, top panels). In addition, direct visualization of WT Src-Cer and Src527F-mCherry in SYF cells demonstrates a concentration of Src-Cer at areas of ECM degradation, with $\mathrm{Src} 527 \mathrm{~F}-\mathrm{mCh}$ localized to the same vicinity (Fig. 5D, bottom panels).

Since the localization of endogenous Src to invadopodia is required for invadopodia maturation, we determined whether catalytically inactive Src could substitute for WT Src and rescue ECM degradation in SYF cells. This result would suggest that two separate and distinct pools of Src (constitutively active and kinaseinactive) are necessary and sufficient for maturation. To test this hypothesis, constitutively active Src $527 \mathrm{~F}-\mathrm{mCherry}$ was coexpressed with a cerulean-tagged kinase-inactive Src (Src295MCer) in SYF cells. Similarly to WT Src, Src295M was largely perinuclear when expressed alone in SYF cells (supplementary material Fig. S4B,C), but was recruited to invadopodia when coexpressed with Src527F (Fig. 5A). However, Src295M failed to rescue invadopodia maturation (Fig. 5B,C), indicating that catalytically inactive Sre does not substitute for WT Src function. Since constitutively active and kinase-dead Src cannot support invadopodia maturation, this suggests that WT Src kinase activity is dynamically regulated to promote invadopodia maturation. Similarly, cell staining for active Src (pY418) localized to preinvadopodia in SYF cells and mature invadopodia in $\mathrm{SYF}^{+/+}$cells (Fig. 5E), ruling out the possibility that catalytically active Src initially drives invadopodia assembly, then is inactivated and remains inactive during maturation. Taken together, these results demonstrate that regulated WT Src kinase activation and inactivation within pre-invadopodia complexes is necessary to govern the downstream signaling events required for invadopodia maturation and ECM degradation.

Regulated Src activity directs cortactin phosphorylation dynamics to control invadopodia maturation

In proposed models of invadopodia maturation, dynamic cortactin tyrosine phosphorylation is required for pre-invadopodia maturation (Oser et al., 2009). To identify a potential mechanism of invadopodia maturation affected by regulated Src activation and inactivation, we assessed the role of Src kinase activity on cortactin phosphorylation. In the SYF/SYF ${ }^{+/+}$system, cortactin phosphorylated on Tyr421 localized to pre-invadopodia and mature invadopodia (Fig. 6A), suggesting that cortactin phosphorylation is important in pre-invadopodia assembly and invadopodia maturation. This is consistent with a well-described role for cortactin in invadopodia maturation in other systems (Artym et al., 2006; Clark et al., 2007; Oser et al., 2009). Stable cell lines were generated expressing endogenous levels of WT cortactin (control) 

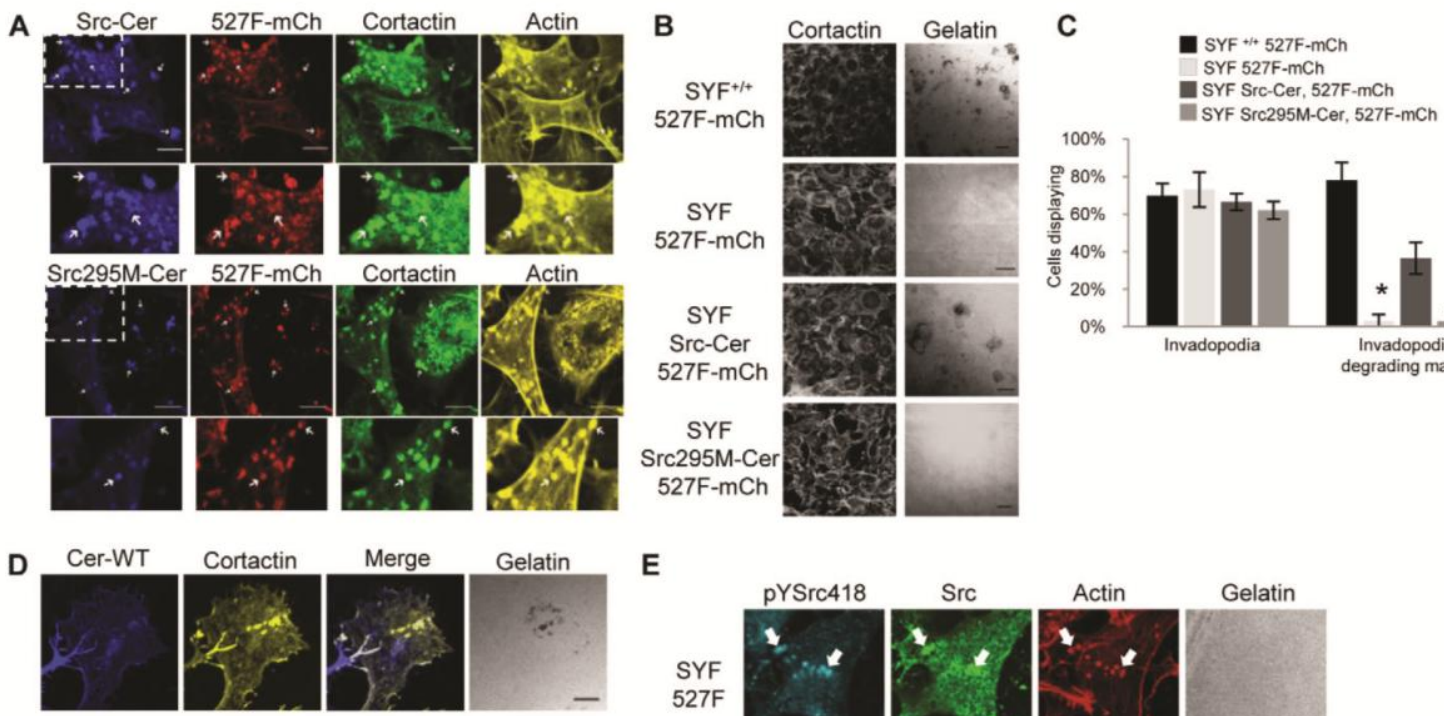

E
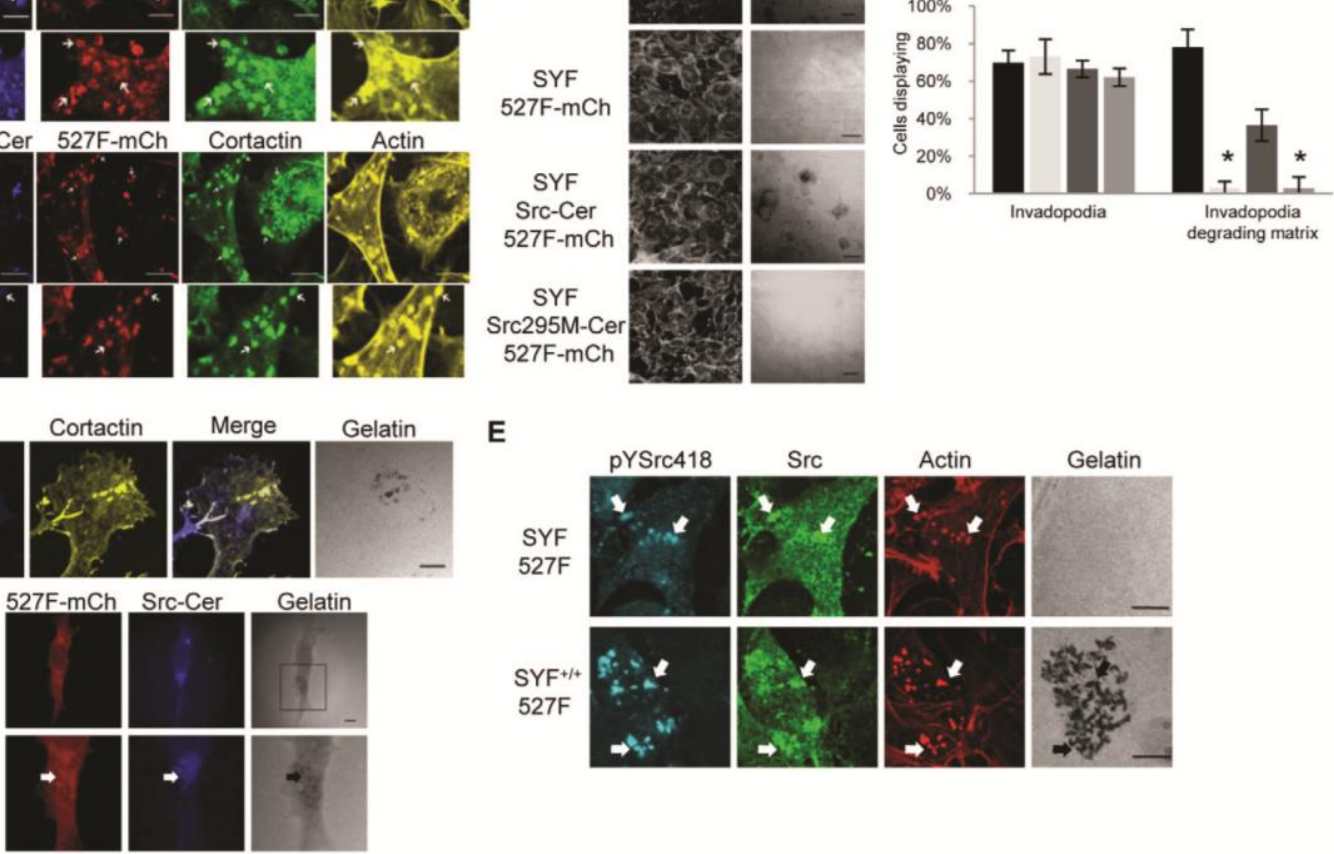

Fig. 5. Expression of regulated Src is necessary for ECM degradation at invadopodia. (A) Confocal imaging of SYF cells coexpressing Src-Cerulean and Src527F-mCherry, or Src-295M-Cerulean and Src527F-mCherry. Cells were immunolabeled with anti-cortactin and Alexa-Flour-647-labeled phalloidin. Dashed boxes represent enlarged image regions shown below. Arrows denote invadopodia. (B) Representative fields of SYF and SYF $\mathrm{F}^{+/+}$cells expressing Src527FmCherry, SYF cells coexpressing Src527F-mCherry and Src-Cerulean, or SYF cells coexpressing Src527F-mCherry and Src295M-Cerulean. Cells were plated on FITC-gelatin-coated coverslips for 24 hours and immunolabeled with anti-cortactin. Areas of gelatin degradation and clearing appear black against the pseudocolored white background. (C) Percentage of cells with forming invadopodia (actin and cortactin aggregates, left), and percentage of invadopodia-forming cells with degraded matrix (mature invadopodia, right) from the experimental conditions shown in B. Data are represented as mean \pm s.d.; $* P \leq 0.05$. (D) Confocal imaging (top) or swept-field imaging (bottom) of SYF cells coexpressing c-Src527F and Src-Cerulean. Cells were plated on FITC-gelatin-coated coverslips and immunolabeled with anti-cerulean (dark blue) and anti-cortactin (yellow) (colocalization appears white in merged image; top panel) or directly imaged for Src527F-mCherry (red) and Src-Cerulean (dark blue) (bottom panels). Arrow indicates colocalization of Src 527F-mCherry and WT Src-Cerulean at sites of gelatin degradation. (E) Confocal imaging of Src activity in SYF and SYF ${ }^{++}$cells expressing Src527F. Cells were incubated on FITC-gelatin-coated coverslips for 24 hours, fixed and immunolabeled with TRITC-phalloidin (red), anti-pY418 Sre (light blue) and anti-Src (green) antibodies. White arrows denote invadopodia; black arrows, matrix degradation. Scale bars: $20 \mu \mathrm{m}$ (B), $10 \mu \mathrm{m}$ (A,D,E).

or a cortactin mutant in which the three Src-targeted tyrosine residues $(421,470,486)$ are mutated to phenylalanine $\left(\mathrm{SYF}^{+/+}\right.$ CortTYM) (Fig. 6B). Endogenous murine cortactin was silenced with siRNA (CortSi, knockdown $>90 \%$ ), resulting in the exclusive expression of WT or mutant human cortactin (Fig. 6C). Wild-type control and CortTYM SYF ${ }^{+/+}$cell lines treated with siRNA to knock down cortactin were transfected with $\operatorname{Src} 527 \mathrm{~F}$ to promote invadopodia formation. Expression of WT human cortactin rescued the inhibitory effects of cortactin knockdown on invadopodia formation, resulting in the formation of mature invadopodia $(\sim 60 \%$ of total cells with invadopodia, Fig. 6D,E). However, only 10\% of $\mathrm{SYF}^{+/+}$CortTYM cells produced degrading invadopodia. Consistent with previous reports (Oser et al., 2009), there were no differences observed in the percentage of cells forming actin or cortactin aggregates in control and CortTYM SYF ${ }^{+/+}$cells, demonstrating that cortactin is targeted to pre-invadopodia independently of tyrosine phosphorylation. However, expression of mutant cortactin in $\mathrm{SYF}^{+/+}$cells completely blocked the upstream function of WT Src, rendering the $\mathrm{SYF}^{+++}$Src527F cells with a degradation profile that was similar to that seen in cells lacking WT Src (SYF Src527F, Fig. 4B,C). These results indicate that there is differential phosphotyrosine signaling in SYF cells with WT Src that supports invadopodia maturation downstream of constitutively active Src.

Cells forming pre- and mature invadopodia have distinct phosphotyrosine signatures

To examine whether distinct tyrosine phosphorylation signatures occur in cells that form pre-invadopodia and mature invadopodia, we conducted a non-biased, comprehensive and quantitative SH2domain screen (Machida et al., 2007) to identify differences in potential phosphotyrosine-binding proteins under conditions of 
A SYF

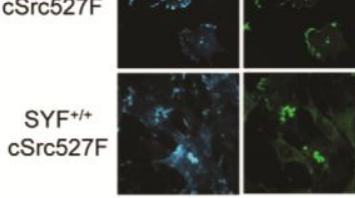

Actin/

D

CortWT

$\mathrm{SYF}^{+/+}$

CortTYM
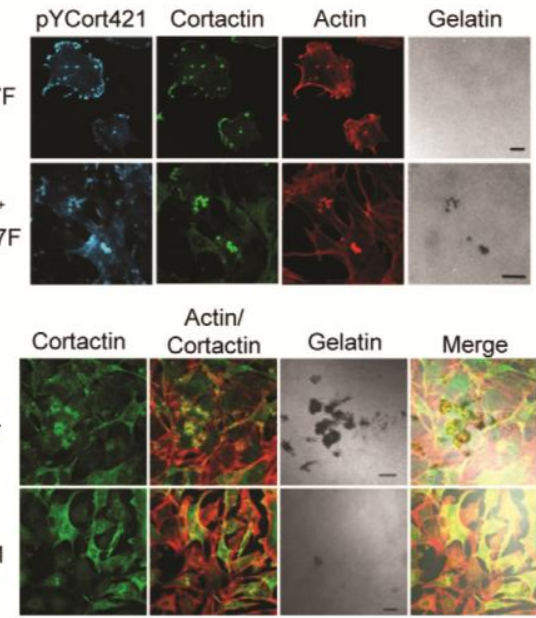

B
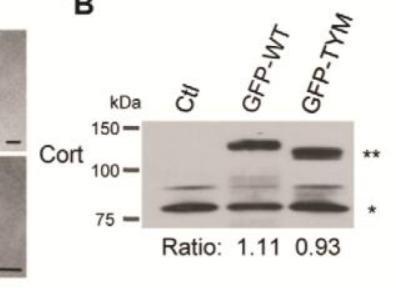

C

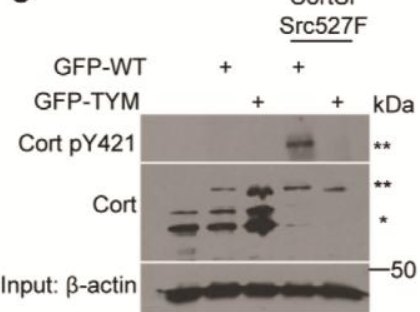

E

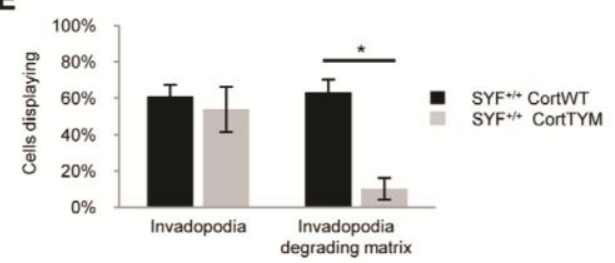

Fig. 6. WT Src regulates cortactin phosphorylation during invadopodia maturation. (A) SYF and SYF ${ }^{+/+}$cells expressing Src527F were incubated on FITCgelatin-coated coverslips for 24 hours, fixed and immunolabeled with TRITC-phalloidin (red), human anti-pY421 cortactin (light blue) and anti-cortactin (green) antibodies. Note that cortactin is phosphorylated in pre-invadopodia and in mature invadopodia, as defined by the absence or presence of matrix degradation. (B) Validation of GFP-tagged cortactin expression in stable cell lines. Cellular extracts from SYF or SYF ${ }^{+/+}$cells stably expressing GFP-CortWT or GFPCortTYM were resolved by SDS-PAGE and immunoblotted with an anti-cortactin (4F11) antibody. *, endogenous cortactin; **, GFP-tagged cortactin (the slight mobility disparity is due to the size difference in AcGFP and EGFP tags); ratios of exogenous (WT and TYM) to endogenous cortactin is depicted under the blot. (C) Clarified lysates from SYF $\mathrm{SF}^{+/+}$GFP-CortWT and SYF ${ }^{+/+}$GFP-CortTYM cells were transfected with Cttn siRNA alone or in combination with Src527F. Cortactin was immunoprecipitated with the anti-cortactin (4F11) antibody, immunocomplexes resolved by SDS-PAGE and immunoblotted with human anti-pY421 cortactin and anti-cortactin $(4 \mathrm{~F} 11)$ antibodies. Total cell lysates were immunoblotted with anti- $\beta$-actin as a loading control. (D) SYF $\mathrm{F}^{+/+}$cells stably expressing human GFP-CortWT or GFP-Cort TYM were transfected with murine cortactin-targeted siRNA to eliminate endogenous cortactin expression. Two days later cells were transfected with Src527F and plated on FITC-gelatin-coated coverslips for 24 hours to promote gelatin degradation. (E) Cells were assessed for the percentage of cells forming invadopodia (actin and cortactin aggregates) and the percentage of invadopodia-forming cells with matrix degradation. Data are represented as mean \pm s.d., ${ }^{*} P<0.05$. Scale bars: $10 \mu \mathrm{m}(\mathrm{A}), 20 \mu \mathrm{m}$ (B).

pre-invadopodia and mature invadopodia formation (Fig. 7; supplementary material Fig. S6). Non-transfected SYF and SYF ${ }^{+/+}$ cells had minimal differences in SH2-domain binding profiles. Expression of Src527F in either cell type enhanced overall SH2 domain signal-binding intensity, indicating a broad increase in phosphotyrosine signaling and creation of new SH2-domain docking sites. This result was anticipated with expression of constitutively active Src. However, cells that form pre-invadopodia (SYF Src527F) had distinct differences in their SH2 binding intensity from cells that form functional mature invadopodia $\left(\mathrm{SYF}^{+/+} \mathrm{Src527 \textrm {F }}\right)$ (bottom row, difference). The variations in binding intensity that arise in SYF and $\mathrm{SYF}^{+/+}$cells expressing Src527F indicate a fundamental mechanistic difference in the concentration of phosphotyrosine binding sites for several SH2domain-containing proteins that are likely to have key roles in invadopodia maturation. Interestingly, tyrosine-phosphorylated cortactin is known to interact with several high-intensity 'hits', including Arg, Abl (Boyle et al., 2007), Fer (El Sayegh et al., 2005), Crk (Bougneres et al., 2004) and Nck (Tehrani et al., 2007), consistent with its role in invadopodia maturation. Experiments to elucidate additional proteins involved in these signaling complexes are currently underway.

\section{Discussion}

In this study, we investigated the role of endogenous or WT c-Src, and the interplay between constitutively active Src and cellular Src in invadopodia formation. Previous studies on Sre in invadopodia formation have exclusively manipulated Src activity in cells containing WT c-Src. Tumor cells that form spontaneous invadopodia presumably have upstream oncogenic signals such as overactivation or overexpression of epidermal growth factor receptor (EGFR), which drives c-Src activation (Xue et al., 2006). Accordingly, increased Src kinase activity (through overexpression of constitutively active Src, or overexpression of WT Src) in tumor cells that form spontaneous invadopodia is associated with increased invadopodia formation and matrix degradation (Artym et al., 2006; Oser et al., 2009). Unlike previous reports (Oser et al., 2009), we did not find that tumor cells overexpressing constitutively active Src had less degradation per invadopodia than spontaneous invadopodia formed in control cells (Fig. 2; supplementary material Fig. S2). However, these differences might be attributed to a much larger capacity for invadopodia formation in UMSCC1/OSC19 cells compared with MtLn3 cells ( $\sim 25$ vs $\sim$ two invadopodia formed in control cells, respectively). In HNSCC cells and Src-null fibroblasts, we propose that constitutively active Src acts as an oncogenic 'trigger' that promotes pre-invadopodia formation, whereas WT Src acts downstream to direct invadopodia stability and maturation. Although models of spontaneous invadopodia formation are invaluable to the field, our finding that Sre cycling is essential for invadopodia maturation could only be completely uncovered through the use of the SYF/SYF ${ }^{+/+}$system.

In this study, we show that WT Src is indispensable for invadopodia maturation driven by elevated Src activity. We hypothesize that distinct, temporally and spatially regulated Src 


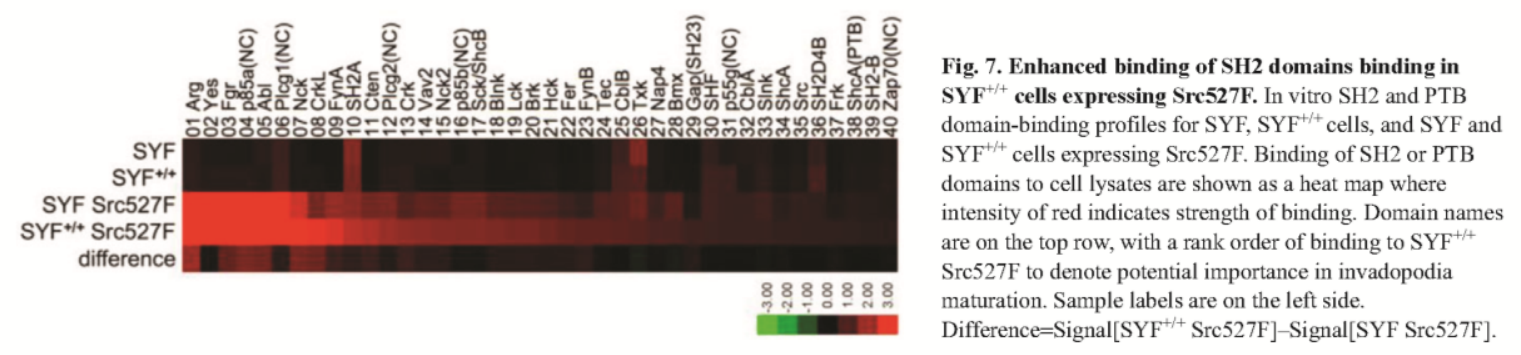

function is necessary to regulate the phosphorylation of cortactin. It is likely that Src regulates other scaffolding proteins that are important in invadopodia stability and maturation, such as dynamin (Caldieri et al., 2008), Tks5 (Seals et al., 2005), IQGAP1 (SakuraiYageta et al., 2008) and paxillin (Bowden et al., 1999). It is possible that Src is activated before its localization with downstream actin-associated substrates in pre-invadopodia. Once preinvadopodia assembly is complete, Src is inactivated [presumably by C-terminal Src kinase (CSK) acting on pY527 (Okada et al., 1991)] and released to regulate additional proteins involved in actin dynamics and MMP delivery to invadopodia. In this way, Src might act as a regulator of several sequentially coordinated proteininteraction events that direct the diverse array of cellular processes at invadopodia, including actin assembly, membrane trafficking and $\mathrm{ECM}$ degradation.

It is known that invadopodia produced by cancer cells and transformed fibroblasts use similar signaling pathways and contain many of the same proteins as podosomes produced by osteoclasts, dendritic cells and macrophages (Linder, 2009). SRC-null osteoclasts have been used to study the role of Src in podosome assembly and dynamics (Destaing et al., 2008). WT Sre and Src527F were both able to restore normal podosome organization, whereas Src295M did not. These data in podosomes support our findings in invadopodia, but since this report did not address the functionality of these cells to degrade ECM, it is not known whether WT Src is needed for maturation in this system. These results are particularly interesting because Src has been suggested to act as a 'molecular switch' to regulate dynamin-Cbl signaling complexes (Bruzzaniti et al., 2005) and as a protein with an important (kinase independent) adaptor function (Bruzzaniti et al., 2009) in osteoclast podosomes. Manipulation of Sre activity in WT osteoclasts also suggest a dual function for $\mathrm{Src}$ in the regulation of actin dynamics through cortactin phosphorylation in podosome assembly and subsequent maturation into more highly organized structures known as sealing zones (Luxenburg et al., 2006).

We identify cortactin as a key regulator of invadopodia maturation downstream of WT Src activity. We show that constituitively active Src is sufficient to target cortactin to preinvadopodia (Fig. 3C, Fig. 4B), but cortactin is targeted independent of its tyrosine phosphorylation (Fig. 6D,E). This is in agreement with previous reports demonstrating that dephosphorylated cortactin acts as a scaffold to recruit Arp2/3, N-WASp and cofilin (Oser et al., 2009). Interestingly, cortactin phosphorylated at Tyr421 is enriched in pre-invadopodia (SYF Src527F) and in matrixdegrading mature invadopodia $\left(\mathrm{SYF}^{+/+} \mathrm{Src527F}\right)$ (Fig. 6A; supplementary material Fig. S3A). In addition, tyrosine phosphorylation of cortactin is required for invadopodia maturation and ECM degradation (Fig. 6D,E) (Ayala et al., 2008; Desmarais et al., 2009; Webb et al., 2007). These data suggest that phosphorylation of cortactin occurs before ECM degradation. However, in our SYF/SYF ${ }^{+/+}$model this initial cortactin phosphorylation is not sufficient to drive maturation in the absence of WT Src, suggesting that cortactin must be dephosphorylated before maturation can occur. Our proposed mechanism of dynamic Src activity on cortactin phosphorylation in invadopodia maturation is consistent with the model recently proposed by the Condeelis laboratory (Oser et al., 2009), whereby cyclical cortactin phosphorylation regulates actin polymerization and invadopodia stabilization.

Here, we show that Src activation and inactivation regulates cortactin phosphorylation during invadopodia maturation. Future studies will be required to determine whether Src kinase is directly responsible for the initial phosphorylation of cortactin to promote invadopodia assembly or in subsequent step(s) to regulate maturation (Huang et al., 1998). Fer (El Sayegh et al., 2005) and Abl family kinases (Boyle et al., 2007) are also present in SYF cells and might act downstream of Src to regulate one or more of these steps. It also follows that tyrosine phosphatases are critically important in the regulation of invadopodia maturation, because cortactin and other possible targets downstream of Src kinase activity in our system require dynamic cycles of phosphorylation and dephosphorylation to drive ECM degradation. Protein tyrosine phosphatase 1B is a good candidate for this process because it is known to regulate Src in invadopodia dynamics (Cortesio et al., 2008) and has subsequently been shown to regulate cortactin dephosphorylation (Stuible et al., 2008). Nonetheless, we show that WT Src kinase is the critical upstream regulator of other downstream kinases and phosphatases important to these processes.

Invadopodia maturation is associated with the delivery of MMPs to invadopodia sites (Artym et al., 2006; Clark et al., 2007). MT1MMP is cited as the key MMP that regulates ECM degradation at invadopodia (Poincloux et al., 2009) and Src kinase activity is known to regulate phosphorylation of MT1-MMP and proteins associated with its trafficking to the cell membrane (Nyalendo et al., 2008; Nyalendo et al., 2007). Future studies should address whether WT Src is necessary to control trafficking to, or activation of MT1-MMP at invadopodia. These studies are further warranted because it has been proposed that a major role of cortactin in invadopodia function involves the targeting and delivery of MMPs to invadopodia to enhance ECM degradation (Clark and Weaver, 2008; Clark et al., 2007).

This report identifies a dominant oncogene that requires the proto-oncogenic complement to reach its full spectrum of transforming functionality. Unlike tumor suppressor proteins, which often require loss of non-mutated alleles, little is known about the potential effect of the presence or absence of proto-oncogenes on their oncogenic counterparts during tumorigenesis. A function in Ras-induced tumorigenesis has been described for Ras proto- 
oncogenes (Singh et al., 2005), where WT Ras has been reported to regulate the functioning of oncogenic Ras with regards to cell proliferation. Our results are consistent with those from the majority of solid human tumors that contain elevated c-Src expression or specific activity due to hyperactivation of upstream regulatory pathways, rather than activating point mutations (Yeatman, 2004). To our knowledge, this is the first description of such a finding. Similar mechanisms might be required for other transforming kinases involved in driving tumor progression.

\section{Materials and Methods}

\section{Cell culture}

HNSCC cell lines UMSCC1, FADU and OSC19 were obtained from Jeffery Myers (M. D. Anderson Cancer Center, Houston, TX). These lines along with UMSCC2 (Thomas Carey, University of Michigan, Ann Arbor, MI), 1483 (David Raben, University of Colorado Denver, Aurora, CO) and MSK921 (Peter Sacks, New York University, New York, NY) were cultured as previously described (Rothschild et al.,

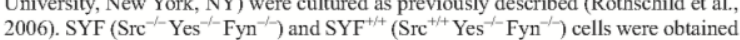
2006). SYF ( $\mathrm{Src}^{-1-} \mathrm{Yes}^{-1-\mathrm{Fyn}^{-1}}$ ) and $\mathrm{SYF}^{+/ 4}\left(\mathrm{Src}^{+/ /} \mathrm{Yes}^{-1-} \mathrm{Fyn}^{-1-}\right)$ cells were obtained
from the American Type Culture Collection. Control non-silencing and human SRCargeted siRNA $\left(5^{\prime}\right.$-AAACTCCCCTTGCTCATGTACTT- $3^{\prime}$ ) were from Dharmacon. OSC19 cells stably expressing vector control or $S R C$-targeted shRNA were created by infection with control or human Src specific lentivirus (Santa Cruz) and cultured according to the manufacturer's instructions. SYF or SYF ${ }^{+/}$cells stably expressing GFP-tagged human cortactin \{WT or Y421F, Y466F, Y482F [triple point mutant (TPM)]\} were created using the Flp-In ${ }^{\text {TM }}$ system (Invitrogen). These lines were transfected with siRNA targeting murine cortactin (Cttn) (5'-GCTTCGAGAGAATGTCTTC- $3^{\prime}$ ) (siCTTN, Dharmacon). For transient transfections, $3 \times 10^{6}$ cells were incubated with $2 \mu \mathrm{g}$ plasmid construct or siRNA. Fibroblast lines were transfected with the Nucleofector I device (Amaxa Biosystems) and HNSCC lines were transfected with TurboFect ${ }^{\mathrm{TM}}$ transfection reagent (Fermentas).

Immunofluorescence labeling and confocal microscopy

Cells were plated on FITC-gelatin (Sigma)-coated coverslips as described (Artym e al., 2006) for 12-24 hours. Cells were fixed with fresh $4 \%$ formaldehyde and permeabilized with $0.4 \%$ Triton X-100 in PBS. Primary antibodies were diluted with $5 \%$ BSA in PBS. Antibodies used were: cortactin 4F11, cortactin EP1922Y (Novus Biologicals), human cort-pY421 (Ammer et al., 2009), Src-pY418 (Biosource), Src GD11 (Upstate), GFP/Cerulean 3E6 (Invitrogen), pTyr-100 (BD Biosciences), Alexa Fluor 405 goat anti-rabbit and Alexa Fluor 647 goat anti-mouse (Molecular Probes). F-actin was labeled with Rhodamine-conjugated phalloidin (Molecular Probes) Cells were mounted in Fluoromount-G (Southern Biotech) and imaged with a Zeiss LSM510 confocal microscope using AIM software (Carl Zeiss MicroImaging). A Nikon Swept-Field using Nikon Elements software (Nikon Instruments) was used for imaging of Src-Cer.

\section{Invadopodia and matrix-degradation assays}

Cells with invadopodia were identified by the presence of at least one actin and cortactin aggregate within the cell $(n \geq 300)$. The number of invadopodia per cell was calculated by counting the number of actin and cortactin aggregates within invadopodia forming cells $(n \geq 150)$. Degradation per cell area was analyzed using ImageJ as described previously (Clark et al., 2007). Cells with invadopodia-degrading matrix were identified by at least one actin and cortactin aggregate colocalizing with matrix were identified by at least one actin and cortactin aggregate colocalizing with
a 'dark hole' that corresponded to degraded FITC-matrix $(n \geq 50)$. Data were pooled from several independent experiments; $n$ represents the number of cells analyzed within each experimental group.

\section{Antibodies and western blotting}

Western blotting of cell lysates was conducted as described (Rothschild et al., 2006). The following antibodies were used: 4F11, Src clone GD11 (Upstate); $\beta$-actin (Calbiochem); Living Colors GFP clone JL-8 (BD); Cort-pY421, Src-pY418 (Biosource); avian Sre clone EC10 (Millipore) and Yes, Fyn (Cell Signaling).

\section{Plasmids}

The Src-GFP linker constructs (WT, 527F, and 295M) were a gift from Margaret Frame (The Beatson Institute for Cancer Research, Glasgow, UK). Substitution of green fluorescent protein (GFP) with cerulean or mCherry fluorescent protein was accomplished through digestion of Src-pEGFP-N1, pmCherry-Cl, and mCerulean$\mathrm{C} 1$ fluorescent vectors with $A g e \mathrm{I}$ and BrsGI. The resulting mCherry and mCerulean fragments ( $700 \mathrm{bp}$ ) were ligated into the GFP-digested Src-containing pEGFP-N1 vector (BD). Temperature-sensitive v-Src (tsLA29) was subcloned from pCMV tsLA29 vector into EGFP-N1 using unique EcoRI and BamHI restriction sites.

\section{Human cortactin constructs.}

A single-stranded primed cDNA library (Invitrogen) was used for cloning human cortactin (CTTN) cDNA. The cDNA was PCR amplified to produce a 965 bp KpnIHincII fragment and a 688 bp HincII-EcoRI fragment. Fragments were ligated into
pcDNA3FLAG2AB that had been digested with KpnI and $E c o$ RI, to generate the 1653 bp full-length $C T T N$ cDNA. The CTTN triple tyrosine mutant (TYM) was generated using the QuikChange kit with primers designed to alter codons 421, 470 and 486 from tyrosine to phenylalanine and confirmed by DNA sequencing. WT and TYM CTTN CDNAs were subsequently amplified as EcoRI-KpnI fragments and subcloned into pEGFP-N1 (WT) or pAcGFP-N1 (TYM). GFP-CTTN WT and TYM fragments were amplified by PCR and subcloned into pEF 5/FRT/V5-D-TOPO (Invitrogen) and stable SYF and SYF ${ }^{+/+}$cell lines generated using the Flp-In system (Invitrogen) according to the manufacturer's instructions.

\section{Immunoprecipitation}

Cells were lysed in NP40 Buffer ( $20 \mathrm{mM}$ HEPES-KOH, pH 7.8, $50 \mathrm{mM} \mathrm{KCl}, 1 \mathrm{mM}$ EDTA and $1 \%$ NP40). Anti-cortactin (4F11, $5 \mu \mathrm{g}$ ) was incubated with $0.5 \mathrm{mg}$ clarified lysates for 2 hours at $4^{\circ} \mathrm{C}$, then incubated with $40 \mu 1$ Protein $\mathrm{A} / \mathrm{G}$ Beads (Thermo Scientific) for 1 hour at $4^{\circ} \mathrm{C}$. Immune complexes were collected by centrifugation, washed twice with NP40 Buffer, separated by SDS-PAGE and western blotted with antibodies as described.

\section{SH2 and PTB binding assay}

SH2 and PTB domain binding assays were performed as described (Dierck et al., 2009; Machida et al., 2007). Briefly, SYF cell lysates were spotted in duplicate on a nitrocellulose membrane in register with the wells of a 96 -well chamber plate Each well was separately incubated with purified GST-SH2 or GST-PTB domains $(\sim 100 \mathrm{nM})$ for 2 hours. Probe binding was detected by enhanced chemiluminescence (ECL) (Perkin Elmer) and digitally captured (Kodak Image Station). Two independent experiments were performed in duplicate, providing four quantifiable data points for each probe. The array images were background-subtracted and the integrated density of each spot was measured using ImageJ (v1.40).

Statistical analysis

Differences in mean values between groups were evaluated using a Student's $t$-tes (two groups) or a one-way ANOVA (multiple groups) followed by Scheffe post-hoc testing.

The contributions of the West Virginia University Microscope Imaging Facility, Mary Babb Randolph Cancer Center, are gratefully acknowledged. This work was supported by National Institute of Health grants P20 RR16440 and R01 DE014578 to S.A.W. Deposited in PMC for release after 12 months.

Supplementary material available online at http://jcs.biologists.org/cgi/content/full/123/22/3923/DC1

\section{References}

Ammer, A. G., Kelley, L. C., Hayes, K. E., Evans, J. V., Lopez-Skinner, L. A., Martin, K. H. and Weed, S. A. (2009). Saracatinib impairs head and neck squamous cel carcinoma invasion by disrupting invadopodia function. J. Cancer Sci. Ther: 1, 52-61.

Artym, V. V., Zhang, Y., Seillier-Moiseiwitsch, F., Yamada, K. M. and Mueller, S. C. (2006). Dynamic interactions of cortactin and membrane type 1 matrix metalloproteinase at invadopodia: defining the stages of invadopodia formation and function. Cancer Res. 66, 3034-3043.

Ayala, I., Baldassarre, M., Giacchetti, G., Caldieri, G., Tete, S., Luini, A. and Buccione, R. (2008). Multiple regulatory inputs converge on cortactin to control invadopodia biogenesis and extracellular matrix degradation. J. Cell Sci. 121, 369-378.

Baldassarre, M., Pompeo, A., Beznoussenko, G., Castaldi, C., Cortellino, S., MeNiven, M. A., Luini, A. and Buccione, R. (2003). Dynamin participates in focal extracellular matrix degradation by invasive cells. Mol. Biol. Cell 14, 1074-1084

Bougneres, L., Girardin, S. E., Weed, S. A., Karginov, A. V., Olivo-Marin, J. C. Parsons, J. T., Sansonetti, P. J. and Van Nhieu, G. T. (2004). Cortactin and Crk cooperate to trigger actin polymerization during Shigella invasion of epithelial cells. Cell Biol. 166, 225-235.

Bowden, E. T., Barth, M., Thomas, D., Glazer, R. I. and Mueller, S. C. (1999). An invasion-related complex of cortactin, paxillin and PKCmu associates with invadopodia at sites of extracellular matrix degradation. Oncogene 18, 4440-4449.

Bowden, E. T., Onikoyi, E., Slack, R., Myoui, A., Yoneda, T., Yamada, K. M. and Mueller, S. C. (2006). Co-localization of cortactin and phosphotyrosine identifie active invadopodia in human breast cancer cells. Exp. Cell Res. 312, 1240-1253

Boyle, S. N., Michaud, G. A., Schweitzer, B., Predki, P. F. and Koleske, A. J. (2007) A critical role for cortactin phosphorylation by Abl-family kinases in PDGF-induced dorsal-wave formation. Curr. Biol. 17, 445-451.

Brabek, J., Constancio, S. S., Shin, N. Y., Pozzi, A., Weaver, A. M. and Hanks, S. K (2004). CAS promotes invasiveness of Src-transformed cells. Oncogene 23, 7406-7415.

Brunton, V. G. and Frame, M. C. (2008). Src and focal adhesion kinase as therapeutic targets in cancer. Curr. Opin. Pharmacol. 8, 427-432.

Bruzzaniti, A., Neff, L., Sanjay, A., Horne, W. C., De Camilli, P. and Baron, R. (2005) Dynamin forms a Src kinase-sensitive complex with $\mathrm{Cbl}$ and regulates podosomes and osteoclast activity. Mol. Biol. Cell 16, 3301-3313.

Bruzzaniti, A., Neff, L., Sandoval, A., Du, L., Horne, W. C. and Baron, R. (2009) Dynamin reduces Pyk2 Y402 phosphorylation and SRC binding in osteoclasts. Mol. Cell. Biol. 29, 3644-3656. 
Buschman, M. D., Bromann, P. A., Cejudo-Martin, P., Wen, F., Pass, I. and Courtneidge, S. A. (2009). The novel adaptor protein Tks4 (SH3PXD2B) is required for functional podosome formation. Mol. Biol. Cell 20, 1302-1311.

Caldieri, G., Giacchetti, G., Beznoussenko, G., Attanasio, F., Ayala, I. and Buccione, R. (2008). Invadopodia biogenesis is regulated by caveolin-mediated modulation of membrane cholesterol levels. J. Cell Mol. Med. 13, 1728-1740.

Chen, W. T. (1989). Proteolytic activity of specialized surface protrusions formed at rosette contact sites of transformed cells. J. Exp. Zool. 251, 167-185.

Clark, E. S. and Weaver, A. M. (2008). A new role for cortactin in invadopodia: regulation of protease secretion. Eur. J. Cell Biol. 87, 581-590.

Clark, E. S., Whigham, A. S., Yarbrough, W. G. and Weaver, A. M. (2007). Cortactin is an essential regulator of matrix metalloproteinase secretion and extracellular matrix degradation in invadopodia. Cancer Res. 67, 4227-4235.

Cortesio, C. L., Chan, K. T., Perrin, B. J., Burton, N. O., Zhang, S., Zhang, Z. Y. and Huttenlocher, A. (2008). Calpain 2 and PTPIB function in a novel pathway with Sre
(a) . Huttenlocher, A. (2008). Calpain 2 and PTPIB function in a novel pathway with Sre
to regulate invadopodia dynamics and breast cancer cell invasion. J. Cell Biol. 180, to regulate

$957-971$.
Desmarais, V., Yamaguchi, H., Oser, M., Soon, L., Mouneimne, G., Sarmiento, C., Eddy, R. and Condeelis, J. (2009). N-WASP and cortactin are involved in invadopodium-dependent chemotaxis to EGF in breast tumor cells. Cell Motil. Cytoskeleion 66, 303-316.

Destaing, O., Sanjay, A., Itzstein, C., Horne, W. C., Toomre, D., De Camilli, P. and Baron, R. (2008). The tyrosine kinase activity of c-Src regulates actin dynamics and organization of podosomes in osteoclasts. Mol. Biol. Cell 19, 394-404

Dierck, K., Machida, K., Mayer, B. J. and Nollau, P. (2009). Profiling the tyrosine phosphorylation state using SH2 domains. Methods Mol. Biol. 527, 131-155.

El Sayegh, T. Y., Arora, P. D., Fan, L., Laschinger, C. A., Greer, P. A., McCulloch, C. A. and Kapus, A. (2005). Phosphorylation of N-cadherin-associated cortactin by Fer kinase regulates $\mathrm{N}$-cadherin mobility and intercellular adhesion strength. Mol. Biol. Cell 16, 5514-5527.

Gatesman, A., Walker, V. G., Baisden, J. M., Weed, S. A. and Flynn, D. C. (2004). Protein kinase Calpha activates c-Sre and induces podosome formation via AFAP-110. Mol. Cell. Biol. 24, 7578-7597.

Guarino, M. (2010). Sre signaling in cancer invasion. J. Cell Physiol. 223, 14-26.

Head, J. A., Jiang, D., Li, M., Zorn, L. J., Schaefer, E. M., Parsons, J. T. and Weed, S. A. (2003). Cortactin tyrosine phosphorylation requires Racl activity and association with the cortical actin cytoskeleton. Mol. Biol. Cell 14, 3216-3229.

Huang, C., Liu, J., Haudenschild, C. C. and Zhan, X. (1998). The role of tyrosine phosphorylation of cortactin in the locomotion of endothelial cells. J. Biol. Chem. 273 phosphorylatio

Linder, S. (2009). Invadosomes at a glance. J. Cell Sci. 122, 3009-3013.

Luxenburg, C., Parsons, J. T., Addadi, L. and Geiger, B. (2006). Involvement of the Src-cortactin pathway in podosome formation and turnover during polarization of cultured osteoclasts. J. Cell Sci. 119, 4878-4888.

Machida, K., Thompson, C. M., Dierck, K., Jablonowski, K., Karkkainen, S., Liu, B. Zhang, H., Nash, P. D., Newman, D. K., Nollau, P. et al. (2007). High-throughput phosphotyrosine profiling using SH2 domains. Mol. Cell 26, 899-915.

Nakahara, H., Mueller, S. C., Nomizu, M., Yamada, Y., Yeh, Y. and Chen, W. T. (1998). Activation of betal integrin signaling stimulates tyrosine phosphorylation of p190R hoGAP and membrane-protrusive activities at invadopodia. J. Biol. Chem. 273, 9-12.

Nyalendo, C., Michaud, M., Beaulieu, E., Roghi, C., Murphy, G., Gingras, D. and Beliveau, R. (2007). Src-dependent phosphorylation of membrane type 1 matrix metalloproteinase on cytoplasmic tyrosine 573, role in endothelial and tumor cell migration. J. Biol. Chem. 282, 15690-15699.

Nyalendo, C., Beaulieu, E., Sartelet, H., Michaud, M., Fontaine, N., Gingras, D. and Beliveau, R. (2008). Impaired tyrosine phosphorylation of membrane type 1-matrix metalloproteinase reduces tumor cell proliferation in three-dimensional matrices and abrogates tumor growth in mice. Carcinogenesis 29, 1655-1664.

Oikawa, T., Itoh, T. and Takenawa, T. (2008). Sequential signals toward podosome formation in NIH-src cells. J. Cell Biol, 182, 157-169.

Okada, M., Nada, S., Yamanashi, Y., Yamamoto, T. and Nakagawa, H. (1991). CSK: a protein-tyrosine kinase involved in regulation of sre family kinases. J. Biol. Chem. 266, 24249-24252.

Onodera, Y., Hashimoto, S., Hashimoto, A., Morishige, M., Mazaki, Y., Yamada, A., Ogawa, E., Adachi, M., Sakurai, T., Manabe, T. et al. (2005). Expression of AMAP1, an ArfGAP, provides novel targets to inhibit breast cancer invasive activities. EMBO J. 24, 963-973.

Oser, M., Yamaguchi, H., Mader, C. C., Bravo-Cordero, J. J., Arias, M., Chen, X. Desmarais, V., van Rheenen, J., Koleske, A. J. and Condeelis, J. (2009). Cortactir regulates cofilin and $\mathrm{N}$-WASp activities to control the stages of invadopodium assembly and maturation. J. Cell Biol. 186, 571-587.

Pichot, C. S., Hartig, S. M., Xia, L., Arvanitis, C., Monisvais, D., Lee, F. Y., Frost, J. A. and Corey, S. J. (2009). Dasatinib synergizes with doxorubicin to block growth, migration, and invasion of breast cancer cells. Br. J. Cancer 101, 38-47.

Poincloux, R., Lizarraga, F. and Chavrier, P. (2009). Matrix invasion by tumour cells: a focus on MT1-MMP trafficking to invadopodia. J. Cell Sci. 122, 3015-3024.

Rothschild, B. L., Shim, A. H., Ammer, A. G., Kelley, L. C., Irby, K. B., Head, J. A., Chen, L., Varella-Garcia, M., Sacks, P. G., Frederick, B. et al. (2006). Cortactin overexpression regulates actin-related protein $2 / 3$ complex activity, motility, and invasion overexpression regulates actin-related protein $2 / 3$ complex activity, motility, and invasi
in carcinomas with chromosome 11 q 13 amplification. Cancer Res, 66, 8017-8025.

Sakurai-Yageta, M., Recchi, C., Le Dez, G., Sibarita, J. B., Daviet, L., Camonis, J., Sakurai-Yageta, M., Recchi, C., Le Dez, G., Sibarita, J. B., Daviet, L., Camonis, J.,
D'Souza-Schorey, C. and Chavrier, P. (2008). The interaction of IQGAP1 with the D'Souza-Schorey, C and Chavrier, P. (2008). The interaction of LGAP1 with the
exocyst complex is required for tumor cell invasion downstream of Cde 42 and RhoA. J. Cell Biol. 181, 985-998.

Sandilands, E., Cans, C., Fincham, V. J., Brunton, V. G., Mellor, H., Prendergast, G C., Norman, J. C., Superti-Furga, G. and Frame, M. C. (2004). RhoB and actin polymerization coordinate Src activation with endosome-mediated delivery to the membrane. Dev. Cell 7, 855-869.

Seals, D. F., Azucena, E. F., Jr, Pass, I., Tesfay, L., Gordon, R., Woodrow, M., Resau, J. H. and Courtneidge, S. A. (2005). The adaptor protein Tks $5 /$ Fish is required for podosome formation and function, and for the protease-driven invasion of cancer cells. podosome formation and
Cancer Cell 7, 155-165.

Singh, A., Sowjanya, A. P. and Ramakrishna, G. (2005). The wild-type Ras: road ahead. FASEB J. 19, 161-169.

Spinardi, L., Rietdorf, J., Nitsch, L., Bono, M., Tacehetti, C., Way, M. and Marchisio, P. C. (2004). A dynamic podosome-like structure of epithelial cells. Exp. Cell Res. 295 360-374.

Stuible, M., Dube, N. and Tremblay, M. L. (2008). PTPIB regulates cortactin tyrosine phosphorylation by targeting Tyr446. J. Biol. Chem. 283, 15740-15746.

Stylli, S. S., Stacey, T. T., Verhagen, A. M., Xu, S. S., Pass, I., Courtneidge, S. A. and Lock, P. (2009). Nck adaptor proteins link Tks5 to invadopodia actin regulation and ECM degradation. J. Cell Sci. 122, 2727-2740.

Summy, J. M. and Gallick, G. E. (2003). Sre family kinases in tumor progression and metastasis. Cancer Melasiasis Rev: 22, 337-358.

Tehrani, S., Tomasevic, N., Weed, S., Sakowicz, R. and Cooper, J. A. (2007). Src phosphorylation of cortactin enhances actin assembly. Proc. Nall. Acad. Sci. USA 104 11933-11938.

Thomas, S. M. and Brugge, J. S. (1997). Cellular functions regulated by Sre family kinases. Annu. Rev. Cell Dev. Biol. 13, 513-609.

Walker, V. G., Ammer, A., Cao, Z., Clump, A. C., Jiang, B. H., Kelley, L. C., Weed, S. A., Zot, H. and Flynn, D. C. (2007). Pl3K activation is required for PMA-directed activation of cSrc by AFAP-110. Am. J. Physiol. Cell Physiol. 293, C119-C132.

Webb, B. A., Eves, R. and Mak, A. S. (2006). Cortactin regulates podosome formation: roles of the protein interaction domains. Exp. Cell Res. 312, 760-769.

Webb, B. A., Jia, L., Eves, R. and Mak, A. S. (2007). Dissecting the functional domain requirements of cortactin in invadopodia formation. Eur. J. Cell Biol. 86, 189-206.

Xue, C., Wyckoff, J., Liang, F., Sidani, M., Violini, S., Tsai, K. L., Zhang, Z. Y., Sahai, E., Condeelis, J. and Segall, J. E. (2006). Epidermal growth factor receptor E., Condeelis, J. and Segall, J. E. (2006). Epidermal growth factor receptor
overexpression results in increased tumor cell motility in vivo coordinately with overexpression results in increased tumor cell motility in vivo
enhanced intravasation and metastasis. Cancer Res. 66, 192-197.

Yamaguchi, H. and Condeelis, J. (2007). Regulation of the actin cytoskeleton in cance cell migration and invasion. Biochim. Biophys. Acta 1773, 642-652.

Yamaguchi, H., Lorenz, M., Kempiak, S., Sarmiento, C., Coniglio, S., Symons, M. Segall, J., Eddy, R., Miki, H., Takenawa, T. et al. (2005). Molecular mechanisms of invadopodium formation: the role of the N-WASP-Arp $2 / 3$ complex pathway and cofilin. J. Cell Biol. 168, 441-452.

Yamaguchi, H., Takeo, Y., Yoshida, S., Kouchi, Z., Nakamura, Y. and Fukami, K (2009). Lipid rafts and caveolin-1 are required for invadopodia formation and extracellular matrix degradation by human breast cancer cells. Cancer Res. 69, 85948602 .

Yeatman, T. J. (2004). A renaissance for SRC. Nat. Rev: Cancer 4, 470-480. 


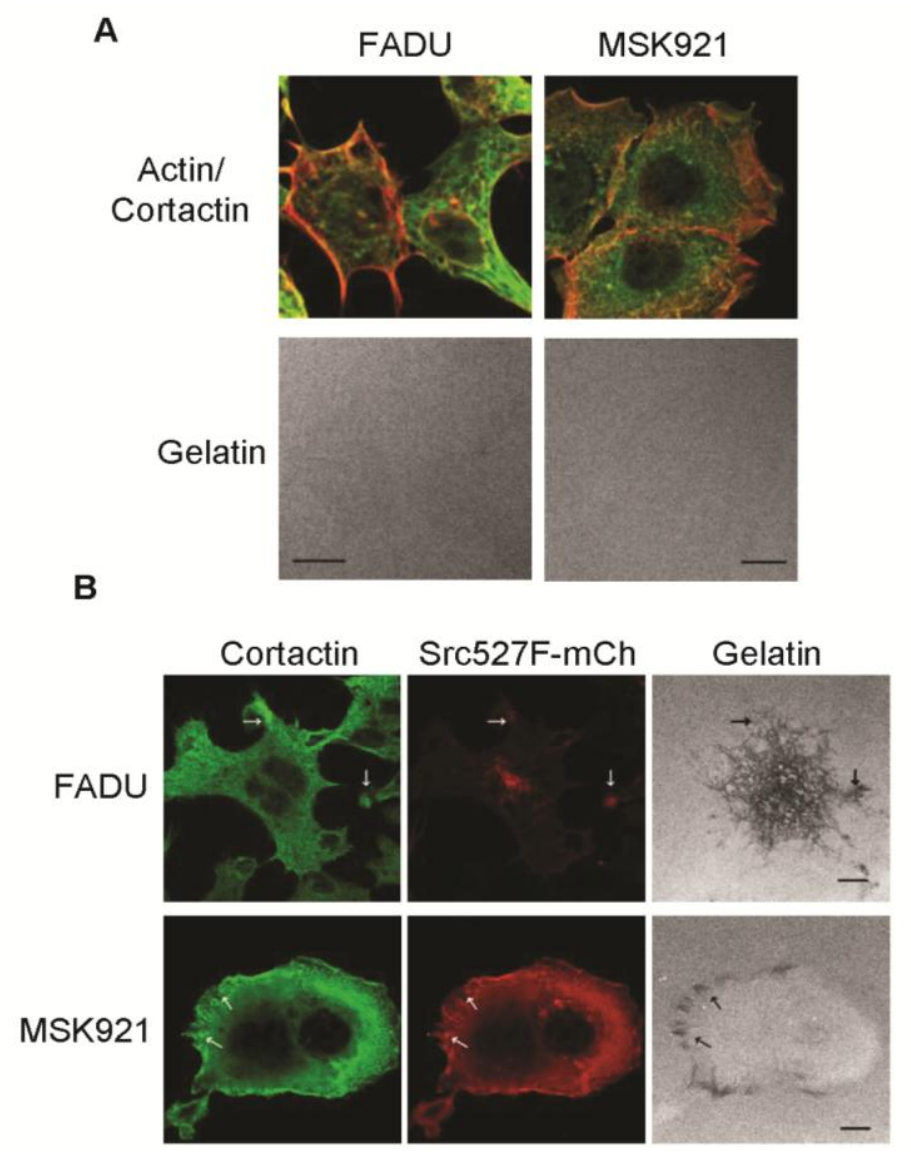

Supplemental Figure 1 Confocal imaging of additional HNSCC cell lines assayed for Src activity and invadopodia-forming capability in Fig 1A. (A) The FADU and MSK921 HNSCC cell lines were incubated on FITC-coated gelatin (white) coverslips for 12 hours, then fixed and labeled with TRITC-phalloidin (red), and cortactin (green) (top panels). Both lines fail to make endogenous invadopodia or degrade matrix. (B) FADU and MSK921 cells transfected with Src527F-mCh (red) were incubated on FITC-coated gelatin (white) coverslips for 12 hours, then fixed and labeled with cortactin (green) (top panels). Src-induced invadopodia are present and identified by the cortactin aggregates (white arrows) that localize with Src527F and the dark holes in the FITC-gelatin (black arrows). Scale bars: $10 \mu \mathrm{m}$.

Kelley LC, et al. J Cell Sci 2010 Nov 15;123(Pt 22):3923-3932. 


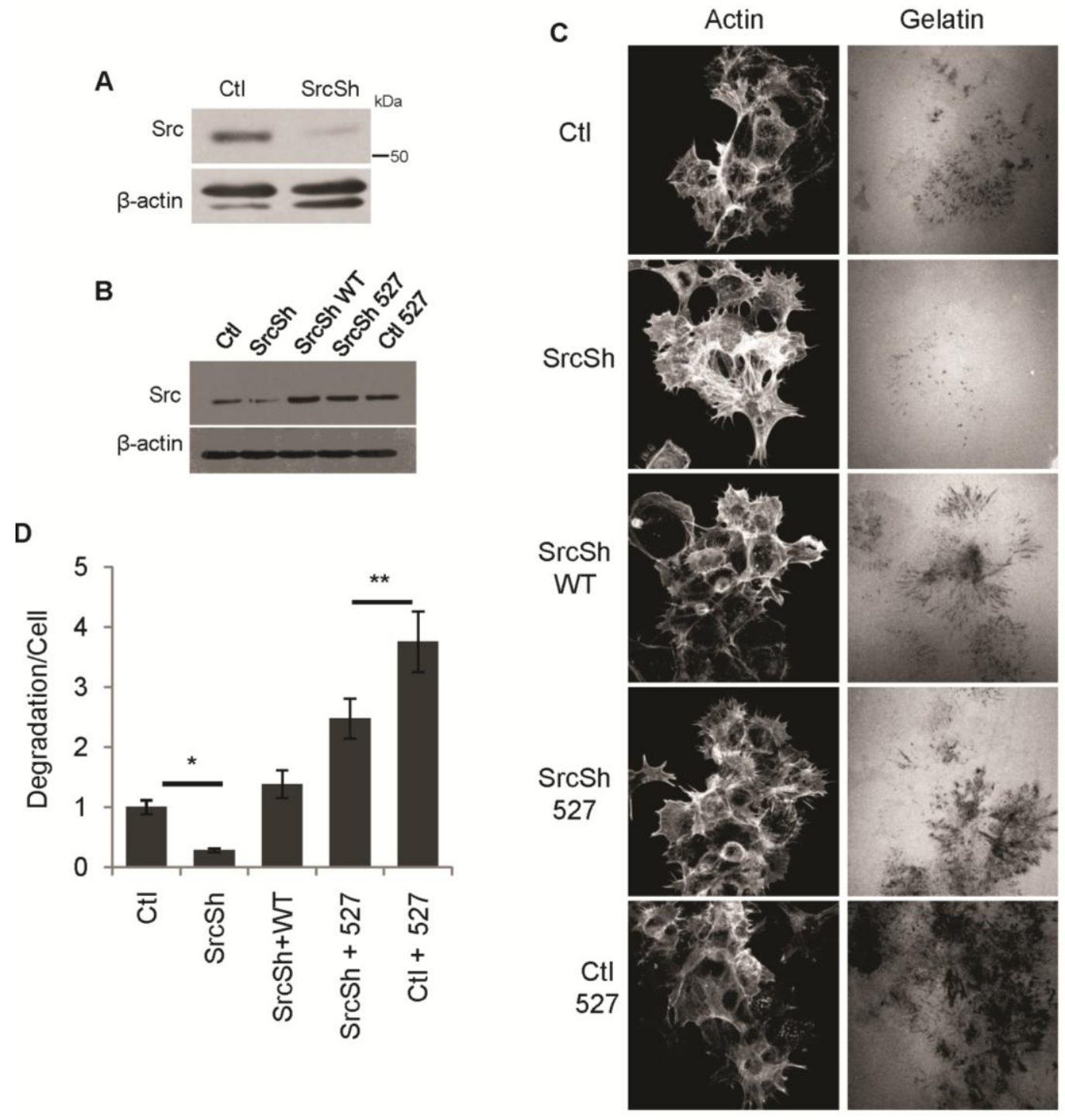

Supplemental Figure 2 Src regulates invadopodia maturation in OSC19 cells. (A) Src protein levels in OSC19 cells treated with with Src shRNA (SrcSh) or a shRNA vector control (Ctl). Expression SrcWT (WT) or Src527F (527) in Ctl and SrcSh cells evaluated by immunoblotting (B) and confocal microscopy (C). Cells were plated on FITC-gelatin-coated (pseudocolored white) coverslips for 10 hours and immunolabeled with TRITC-phalloidin (red). Scale bars: $10 \mu \mathrm{m}$. (D) The effect of Src expression on percentage of cells displaying invadopodia, the number of invadopodia per cell, and the amount of matrix degradation per cell were examined. Data are represented as mean \pm s.e.m., groups are statically different $\left({ }^{*} P \& \lambda \mathrm{T} ; 0.01,{ }^{* *} P \& \lambda \mathrm{T} ; 0.05\right)$.

Kelley LC, et al. J Cell Sci 2010 Nov 15;123(Pt 22):3923-3932. 

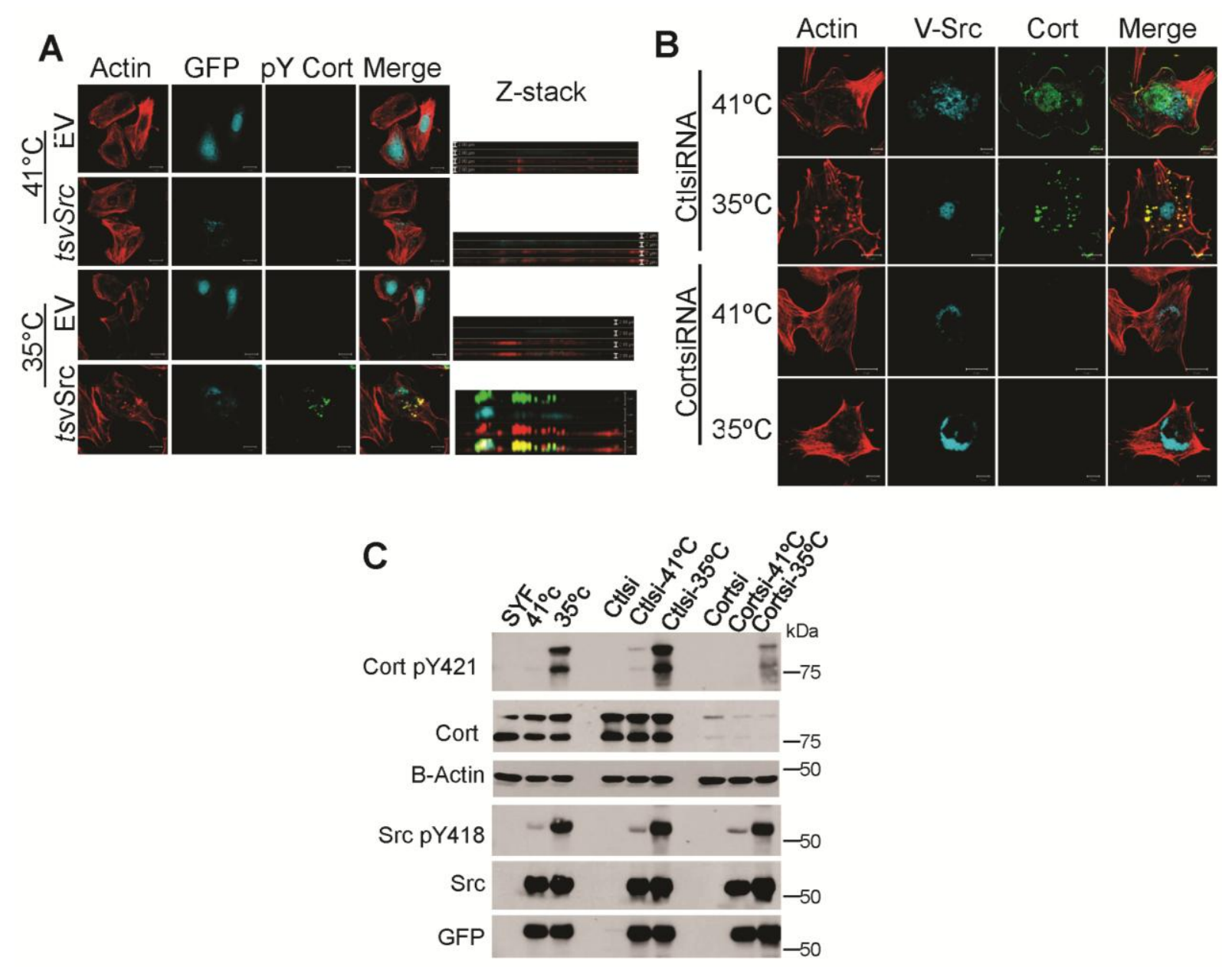

Supplemental Figure 3 (A) Invadopodia are formed in cells expressing tsLa29-GFP at the permissive temperature. SYF cells transfected with empty GFP vector (EV) or with tsLa29 tagged with GFP on the C-terminus (tsLa29-GFP) were incubated at $41^{\circ} \mathrm{C}$ or $35^{\circ} \mathrm{C}$. Cells were fixed, permeabilized, and dual-labeled with TRITC-phalloidin and a phospho-specific antibody for cortactin Tyr421. Cells were visualized by confocal microscopy through 2D and 3D (z-stack) sectioning. (B) Silencing of cortactin with siRNA prevents invadopodia formation. SYF cells were transfected with cortactintargeted or control siRNA $(\mathrm{Ctl})$ and incubated at $37^{\circ} \mathrm{C}$ for 2 days. Cells were then transfected with tsLa29-GFP and the experiment proceeded as described in A. Cells were fixed, permeabilized, and immunolabeled with TRITC-phalloidin and an anticortactin (4F11) antibody. Invadopodia fail to form in cells treated with cortactin siRNA. (C) Clarified cell lysates $(30 \mu \mathrm{g})$ from cells transfected with mock siRNA or siRNA to knock down cortactin, alone or in combination with tsLa29-GFP, were incubated at $41^{\circ} \mathrm{C}$ or $35^{\circ} \mathrm{C}$, resolved by SDS-PAGE and immunoblotted with anti-Src-pY418, anti-Src (EC10), anti-cortactin (4F11), anti-cortactin-pY421, anti-GFP (JL8) and anti- $\beta$-actin antibodies. Scale bars: $10 \mu \mathrm{m}$.

Kellev LC, et al. J Cell Sci 2010 Nov 15:123(Pt 22):3923-3932. 
A

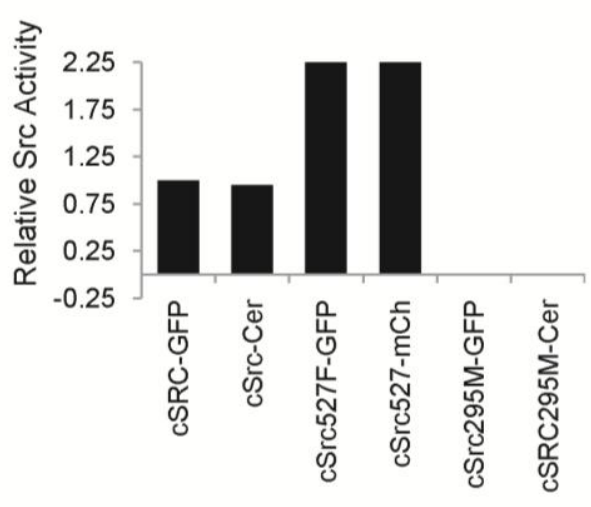

B

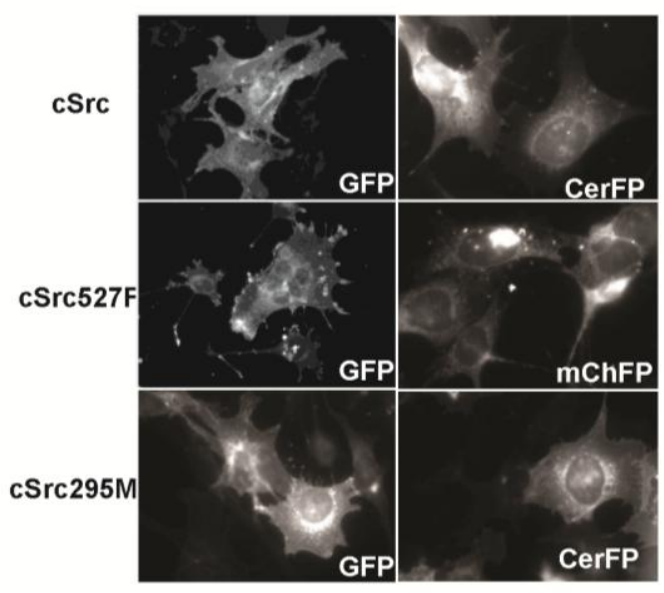

C

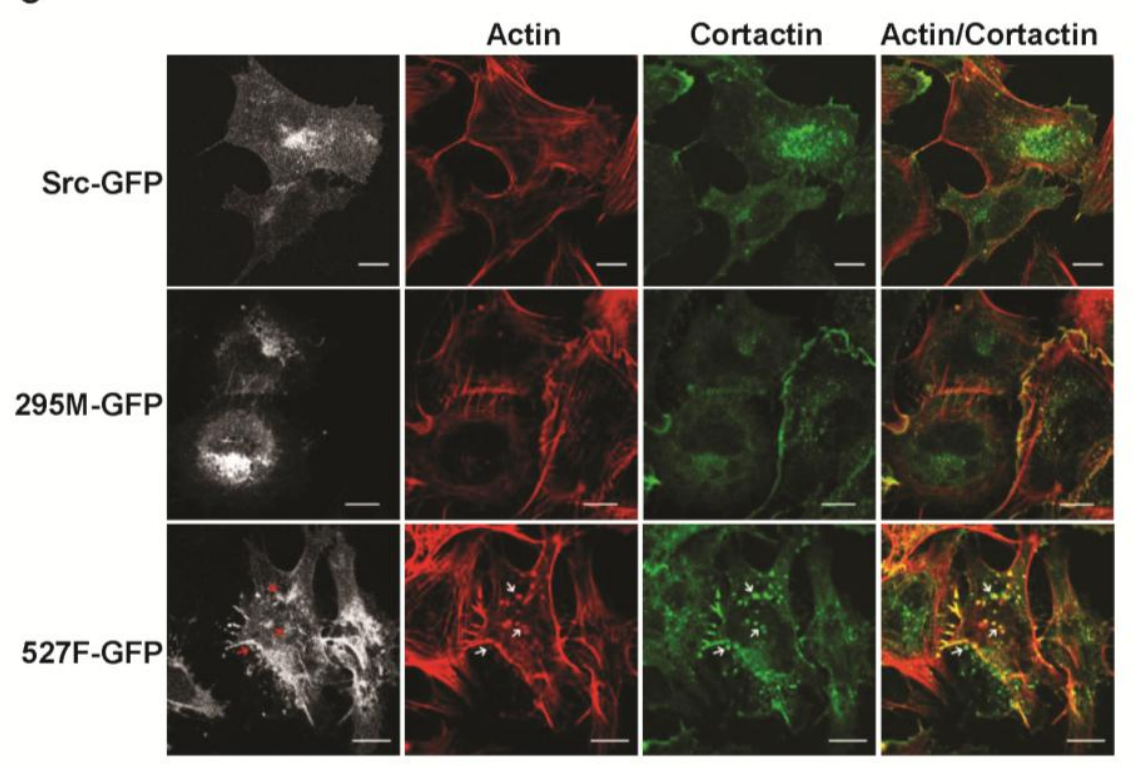

Supplemental Figure 4 Characterization of fluorescent protein-tagged Src constructs. (A) Determination of relative Src kinase activity. SYF cells expressing Src-GFP, Srccerulean, Src527F-GFP, Src527F-mCherry, Src295M-GFP, or Src295M-cerulean were lysed, resolved by SDS-PAGE and immunoblotted with anti-Src-pY418, anti-Src, and anti- $\beta$-actin antibodies. Quantification of the relative Src phospohorylation in transfected SYF cells was conducted using densitometry. Src-pY18 protein expression was normalized to total Src protein levels. (B) Swept-field imaging of fluorescently labeled Src constructs. Fixed cells were imaged for expression and localization of GFP, mCherry, and cerulean-tagged Src fluorescent proteins by direct fluorescence. GFP, green fluorescent protein; CerFP, cerulean fluorescent protein; mChFP, mCherry fluorescent protein. (C) Confocal imaging of GFP-tagged SrcWT, Src295M, and Src527F. Transfected cells expressing the indicated Src constructs were fixed, permeabilized and immunolabeled with TRITC-phalloidin and the anti-cortactin (4F11) antibody. Arrows indicate invadopodia in cells expressing Src527F. Scale bars: $10 \mu \mathrm{m}$. Kelley LC, et al. J Cell Sci 2010 Nov 15;123(Pt 22):3923-3932. 
A

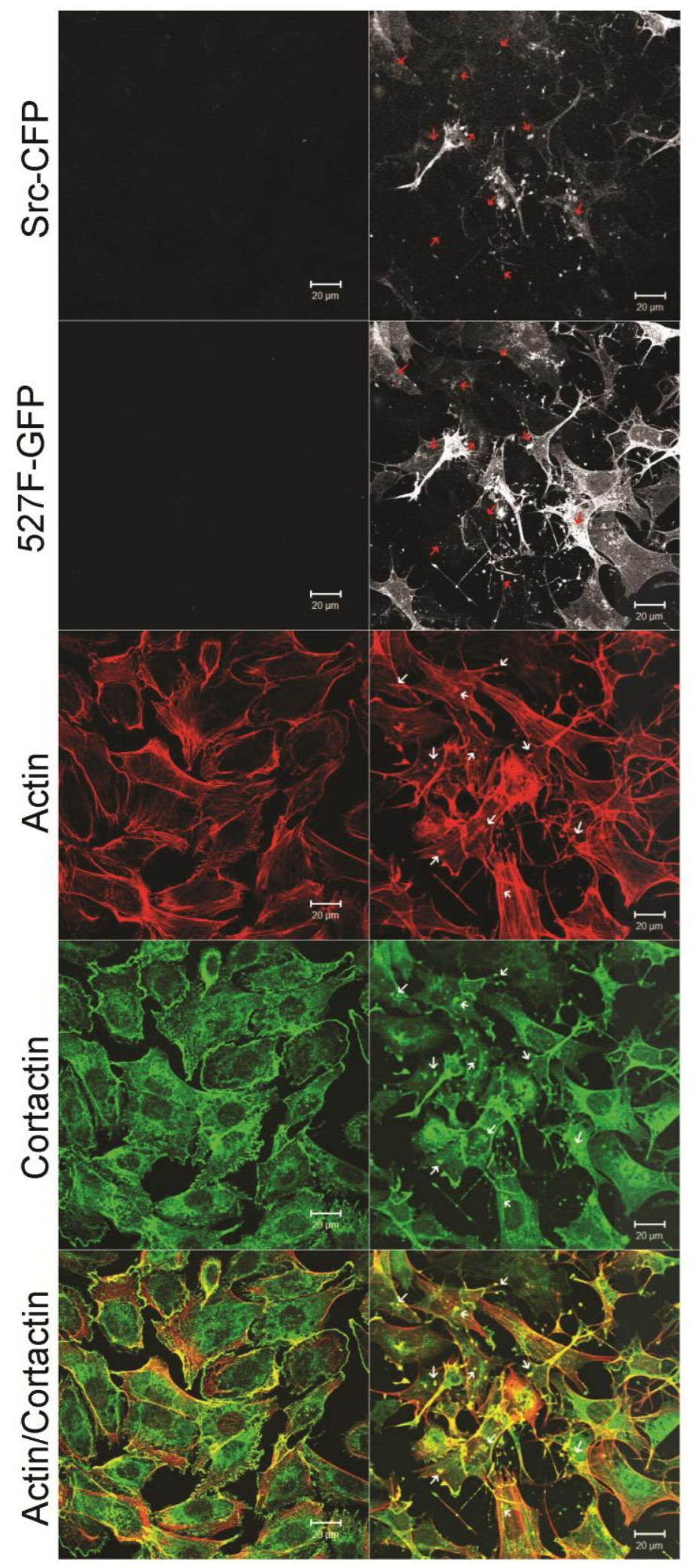

B

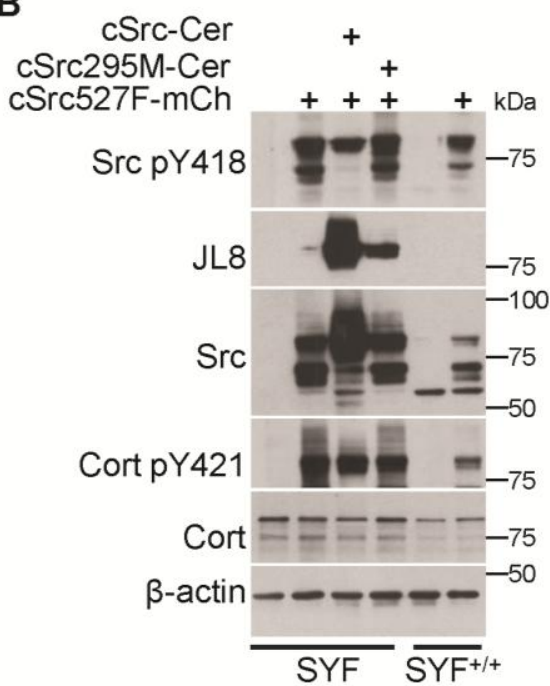

Supplemental Figure 5 Cotransfection and localization of fluorescent protein tagged Src constructs. (A) Representative images of non-transfected SYF cells (left) and SYF cells coexpressing WT Src tagged with Cerulean fluorescent protein (Src-Cer) and GFP-tagged Src527F (527F-GFP) (right). Cells were fixed, permeabilized and immunolabeled with TRITC-phalloidin and anticortactin (4F11) antibody. Arrows denote invadopodia in the 527F-GFP-transfected cells. Scale bar: $20 \mu \mathrm{m}$. (B) SYF and $\mathrm{SYF}^{+/+}$cells expressing cSrc527F-mCherry alone or in combination with Src-cerulean or Src295M-cerulean, were lysed and resolved by SDSPAGE and immunoblotted with anti-Src-pY418, anti-Src, antiGFP or anti-cerulean (JL8, does not recognize mCherry), antiactin, anti-cortactin-pY421 and anti-cortactin (4F11) antibodies.

Kellev LC, et al. J Cell Sci 2010 Nov 15:123(Pt 22):3923-3932. 
Supplemental Figure 6

Comprehensive $\mathrm{SH} 2$ and PTB binding assay. An in vitro binding assay was performed using 91 GST

SH2 domains and three GST PTB domains, representing nearly the full complement of human phosphotyrosine-binding domains. GST and mutated Abl SH2 domain (Abl $R>K$ ) were used as negative controls. SH2 binding to SYF cell lysate was determined by densitometric quantification of digitally captured chemiluminescence images. The mean raw binding intensities with s.e.m. from two independent experiments are shown. Domains are ordered from left to right by their binding intensity to $\mathrm{SYF}^{+/+}$cells expressing Src527F to reflect rank order relevance for invadopodia maturation.

Kelley LC, et al. J Cell Sci 2010 Nov 15;123(Pt 22):3923-3932.

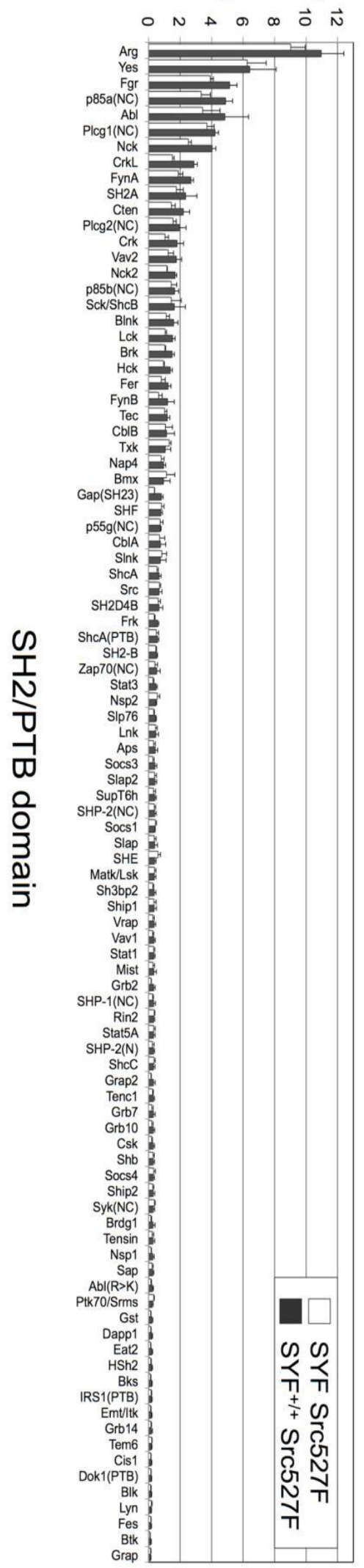




\section{Karen E. Hayes}

CONTACT

INFORMATION

EDUCATION

RELATED EMPLOYMENT

DOCTORAL DISSERTATION
401 Ontario Ave, Point Marion, PA 15474

Home: (724) 725-0729 - Work: (304) 293-3090

kehayes@hsc.wvu.edu

West Virginia University School of Medicine

Morgantown, WV

2004-present

PhD candidate, Cancer Cell Biology

Edinboro University of Pennsylvania

1997

Edinboro, PA

B. S., Biology, Cum Laude, Department of Biology

Free-Col Laboratories, A Division of Modern

Industries, Meadville, PA

Laboratory Analyst

1999-2004

Areas of responsibilities include maintaining bioassay cultures, reference toxicant analysis, and WET test analysis, wet chemistry analysis utilizing spectrophotometer, ion selective electrodes, titrations and distillation units. Other responsibilities included assisting in the microbiology department and reviewing other chemist's analytical reports.

Abelson Kinase Based Regulation of Tumor Cell Invasion in HNSCC

Dissertation Defense Date: December 11, 2012

Laboratory techniques learned and utilized included biochemical protein analysis and purification (Western blotting, immunoprecipitation, affinity precipitation, recombinant protein production, gelatin zymography, ELISA); cellular migration, adhesion, and invasion assays (ECIS, wound healing assays, gelatin degradation assay, Boyden chamber based assays, 3DCollagen I invasion assay); kinase assays; DNA cloning/mutagenesis; fluorescent/confocal microscopy; flow cytometry; tissue/cell culture techniques (maintaining cell lines, cellular transfection, lentivirus preparation and subsequent cellular 
infection); and orthotopic xenografts of oral squamous cell carcinoma examining perineural invasion and lymph node metastasis.

PUBLICATIONS Ableson Kinases Negatively Regulate Invadopodia Function and Invasion in Head and Neck Squamous Cell Carcinoma by Inhibiting an HB-EGF Autocrine Loop, Karen E. Hayes*, Elyse L. Walk, Amanda Gatesman Ammer, Laura C. Kelley, Karen H. Martin, and Scott A. Weed Oncogene 2012 Nov; 1(2) online pub doi.1038/onc.2012.513.

Quantitative Measurement of Invadopodia-mediated Extracellular Matrix Proteolysis in Single and Multicellular Contexts, Karen $\mathrm{H}$. Martin*, Karen E. Hayes, Elyse L. Walk, Amanda Gatesman Ammer, Steven M. Markwell and Scott A. Weed J. Vis Exp. (JOVE) 2012 Aug 27;66.

Multi-photon Imaging of Tumor Cell Invasion in an Orthotopic Mouse Model of Oral Squamous Cell Carcinoma, Gatesman Ammer $A^{*}$, Hayes KE, Martin KH, Zhang L, Spirou GA, and Weed SA. J. Vis Exp. (JOVE) 2011 Jul; 53:2941.

Revisiting the ERK/Src Cortactin Switch, Hayes KE*, Kelley LC*, Ammer AG*, Martin $\mathrm{KH}$, and Weed SA Commun. Integr. Biol. 2011 Mar;4(2):205-207.

Further Insights into Cortactin Conformational Regulation, Jason V. Evans $^{*}$, Laura C. Kelley, Karen E. Hayes, Amanda Gatesman Ammer, Karen H. Martin and Scott A. Weed Bioarchitecture 2011 Jan;1(1):21-23.

Oncogenic Src Requires a Wild-type Counterpart to Regulated Invadopodia Maturation, Kelley LC*, Ammer AG, Hayes KE, Martin KH, Machida K, Jia L, Mayer BJ, and Weed SA J. Cell Sci. 2010 Nov 15;123:3923-3932.

Cortactin Phosphorylated by ERK1/2 Localizes to Sites of Dynamic Actin Regulation and Is Required for Carcinoma Lamellipodia Persistence, Karen E. Hayes*, Laura C. Kelley*, Amanda Gatesman Ammer*, Karen H. Martin, and Scott A. Weed PLoS One 2010 Nov;5(11):e13847.

Sarcatinib Impairs Head and Neck Squamous Cell Carcinoma Invasion by Disrupting Invadopodia Function, Hayes KE* Ammer AG*, Kelley LC*, Evans JV, Lopez-Skinner LA, Martin KH, 
Frederick B, Rothschild BL, Raben D, Elvin P, Green TP, Weed SA $J$ Cancer Sci Ther. 2009 Nov 30;1(2):52-61.

*Denotes First Authors

ABSTRACTS The $4^{\text {th }}$ Biennial National IDeA Symposium, Washington, DC, June 2012, Ableson Kinases Negatively Regulate Invadopodia and Invasion in HNSCC, Karen Hayes and Scott Weed.

The American Society for Cell Biology 50th Annual Meeting, Philadelphia, PA, December 2010, $c-A b l$ is a Novel Negative Regulator of Invadopodia Activity, Karen Hayes, Karen H. Martin, Laura Kelley, Mark Culp and Scott Weed.

EJ Van Liere Convocation and Research Day, Morgantown, WV, April 2009, A c-Abl -Cortactin Complex Regulates Invadopodia Activity, Karen E. Hayes and Scott A. Weed.

4th Annual West Virginia COBRE/INBRE Conference, Morgantown, WV, October 2008, A c-Abl-Cortactin Complex Regulates Invadopodia Activity, Karen E. Hayes, Karen H. Martin and Scott A. Weed.

The 3rd Annual West Virginia COBRE/IMBRE Conference, Charleston, WV, November 2007, Role of $c-A b l$ in Cortactin Phosphorylation, Karen E. Hayes, Laura F. Gibson, Mark Auble, Ann Marie Pendergast, Scott A. Weed.

EJ Van Liere Convocation and Research Day, Morgantown, WV May 2007, Role of c-Abl in Cortactin Phosphorylation, Karen E. Hayes, Laura F. Gibson, Mark Auble, Ann Marie Pendergast, Scott A. Weed.

The American Association for Cancer Research 98th Annual Meeting, Los Angeles, CA, April 2007, Role of c-Abl in Cortactin Phosphorylation, Karen E. Hayes, Laura F. Gibson, Mark Auble, Ann Marie Pendergast, Scott A. Weed.

PROFESSIONAL Organized and hosted guest speaker, Dr Donna Webb, for West DEVELOPMENT Virginia University Cell Biology Training Seminar, May 2010.

Imaging Ethics, Acquisition, Post-Processing and Quantification Course, taught by Jerry Sedgewick, June 2012. 
MEMBERSHIPS AACR (American Association for Cancer Research) 2010-2012 ASCB (American Society for Cell Biology) 2006-2012 West Virginia University Cytoskeletal Signaling Group 2008-2010 West Virginia University Cell Biology Training Consortium

2009-2012 